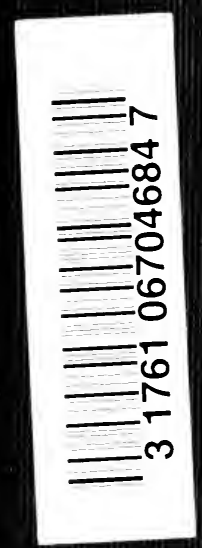




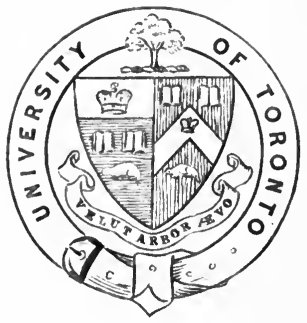

㹱resented to

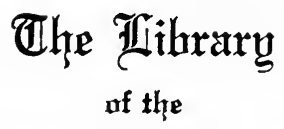

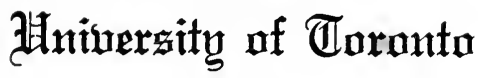

hy

DR. E.S. RYERSON 
,

.

.

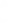


Digitized by the Internet Archive in 2007 with funding from Microsoft Corporation 


\section{An Outline}

\section{of \\ Genito - Urinary Surgery}

By

George Gilbert smith, M. D., F. A. C. S.

Genito-urinary Surgeon tc Uut-patients, Massachusetts (ieneral llospital; Assist'unt Visiting Surgeon, Collis P. Huntington Menorial Hospital; Captain Medical Corps, I. S. A.; Follow Ameriean College of Surgeons; Member of the Aneriean Association of (ienito-urinary Surgeons and of the American Crologicall Issociation

Authority to publish

Granted by the Surgeon-Generai, U. S. A.

Illustrations by H. F. Aitken

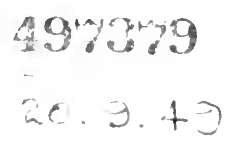

Philadelphia and London

W. B. Saunders Company

1919 
Copyright, 1919, by W. B. Saunders Company

PRINTED IN AMERICA

PRE 68 OF

W. B. GAUNDERs COMPANY

PHILADELPHIA 
TO

HUGH CABOT 



\section{FOREWORD}

THIs book has been written with the idea of presenting to students and to general practitioners the important points in the symptomatology and pathology, of genito-urinary diseases. The tratment of these conditions, in so far as it deals with medicne or with minor surgery, is given in full; when major surgery is indicated, enough of the technic is described to give the medical reader an intelligent opinion as to the nature of the operation, its aims and its dangers.

The methods and technic described are by no means the only ones possible, but they are measures which I in my own experience have found practicable. The case histories are almost entirely from my own cases, either in private practice or at the Massachusetts General Hospital.

The articles referred to at the end of each chapter have been selected because of their value as original contributions, or because they represent the views of men of authority upon the subject under discussion.

The drawings were made by Mr. H. F. Aitken from actual operations and from pathologic specimens. I take this opportunity to express my great appreciation of his interested and intelligent coöperation.

\section{George Gilbert Smith.}

99 Commonwealth Avenue, Boston, Mass.,

April, 1919. 



\section{CONTENTS}

CHAPTER I

PAGE

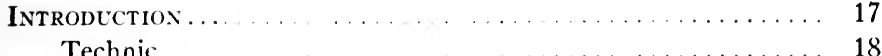

Asepsis in Urethral Instrumentation, 20-Equipment, 22.

Endoscopy and cystoseopy . . . . . . . . . . . . . 24

Endoscopy, 24-. Interior Urethroscopy, 26-P'osterior Urethroscopy, 26-Cystoscopy, 27.

CIIAPTER II

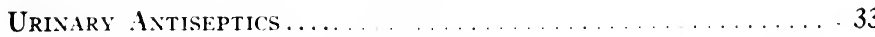

The Balsamics, 33-Hexamethylenamin, 34-Other Drugs, 38.

CHAP'TER III

Urinalysis. Tests of ReNal Function . . . . . . . . . . . . 39

Urinalysis .... . . . . . . . . . . . . . . . . . 39

Albumin, 40-Sugar, 40-Sediment, 40-Specimens Stained for the Tubercle Bacillus, 41-Urethral and Prostatic Secretion, 42-Cionococcus Complementfixation Test, 43.

Renal Function Tests . . . . . . . . . . . . . . . . .

Technic, 45.

CHAPTER IV

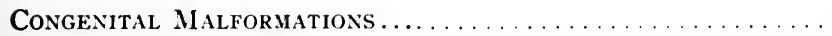

Kidney and Ureter, 49-Bladder, 50-Fistula and Cyst of the Urachus, 54-Exstrophy of the Bladder, 54-Epispadias, 55-Hypospadias, 56-Undescended Testis, 57.

\section{CHAPTER V}

Diseases of the Penis.

Chancroid, 61-Bubo, 62-Chancre, 62-Venereal Warts (Condylomata), 63-Balanitis, 64-Phimosis, 64-Circumcision, 65-Paraphimosis, 70-Cancer of the Penis, 71. 


\section{CHAPTER VI}

Diseases of the Urethra.............................

Gonorrhea, 73-Chronic Urethritis, 87-Non-specific Urethritis, 90.

\section{CHAPTER VII}

Diseases of the Urethra (Contimued)................ 95

Stricture, 95.

\section{CHAPTER VIII}

Diseases of THE URETHRa IN WoMen

Acute Urethritis, 103-Chronic Urethritis, 103-Stricture of the Urethra, 105-Caruncle, 105-Prolapse of the Urethra, 106-Urinary Incontinence, 106.

\section{CHAPTER IX}

Infections of the Prostate and Seminal Vesicles.

Acute Infections, 107-Subacute and Chronic Prostatitis and Vesiculitis, 114-Chronic Non-specific Prostatitis and Vesiculitis, 118-Cure of Gonococcus Infections, 120Tuberculosis of the Prostate and Vesicles, 123.

\section{CHAPTER $\mathrm{X}$}

Prostatic Obstruction.

Symptoms, 125-Diagnosis, 128-Management of Prostatics, 132-Cystotomy, 138-Prostatectomy, 146-Postoperative Treatment, 155-After-treatment of Prostatectomy, 156-The Small Fibrous Prostate, 157-Cancer of the Prostate, 158.

\section{CHAPTER XI}

Diseases of Scrotum and Testicle.................. 163

Hydrocele; Spermatocele; Hematocele. . . . . . . . . . . . . . 163

Diseases of the Spermatic Cord .................... 174

Hydrocele of the Cord, 174-Varicocele, 175-Torsion of the Spermatic Cord, 176.

Diseases of the Testicle.

Trauma of the Testicle, 178-Epididymo-orchitis of Mumps, 178-Abscess of Testicle, 181-Tuberculosis of the Testis, 182-Syphilis of the Testicle, 182Neoplasm of the Testicle, 183-Epididymitis, 183. 


\section{CHAPTER XII}

Diseases of THE Bladder ..................... 188

Cystitis, 189-Encrusted Bladder, 195-Chronic Cystitis, 196 Syphilis of the Bladder, 203-Bilharzia Infection, 203Tumor, 204-Vesical Calculus, 212-Rupture of the Bladder, 213.

\section{CHAPTER XIII}

Diseases of Kidneys ant Ureters..................... 216

Urinary Abnormalities, 216-Pyuria, 219-Pain, 219-Disturbances of Urination, 220-Fever, 220-Abdominal Tumor, 220-Renal Insufficiency, 225-Essential Hematuria, 226-Decapsulation in Nephritis, 228-Nephroptosis, 228-Renal Infections, 232-Pyelitis of Pregnancy, 235-Pyelitis of Childhood, 237-Sequelæ of Pyelitis, 238 -Renal Retention, 243-Renal and Ureteral Stone, 245 -Renal Tumor, 255-Rupture of the Kidney, 257.

\section{CHAPTER XIV}

Genito-urinary Tuberculosis. .

Kidney and Bladder, 261-Tuberculous Epididymitis, 265.

\section{CHAPTER XV}

Gonococcus Infection in the Female.

Vulvovaginitis of Little Girls, 272-Vulvovaginitis in Adult, 276.

\section{CHAPTER XVI}

IMPOTENCE AND STERILITY 278

Sterility, 282. 
- 


\section{AN OUTLINE 0F GENIT0-URINARY SURGERY}

\section{CHAPTER I \\ INTRODUCTION}

THE management of genito-urinary disease presents to the medical profession many problems. Not least of these is the problem of bringing about between the general practitioner and the specialist that co-operation which is so essential to the welfare of the patient. Obriously, the man in general practice cannot be expected to make those diagnoses which require special instruments and the skill necessary to their use. No more can he be expected to send all his genito-urinary cases to the specialist; indeed, in many smaller communities the services of a urologist may be quite difficult to obtain.

Certain conditions, such as hematuria, clearly require these services, no matter how inaccessible they may be; other conditions may be treated with perfectly satisfactory results by the general practitioner, provided he will make use of those measures of diagnosis and treatment which are within his powers. In this he is aided by his knowledge of the patient's temperament, general health, and social and economic condi- 
tions, and in many instances his advice is more seriously accepted because of the confidence which past relations with the patient have engendered. Yet all of these advantages, valuable though they are, become as nought if he is ignorant of the actual pathology underlying his patient's condition.

Not only must he know the pathology, but he must have a very definite idea of the object of treatment and the ends which he may hope to attain. In the management of these cases, particularly when they are ambulatory and not incapacitated by severe symptoms, there is considerable temptation to slip into an aimless, visit-to-visit policy. This must be guarded against, first, by the establishment of an exact diagnosis, then by the institution of definite measures to correct whatever is wrong. If one feels himself unable to achieve either of these aims, he should seek help from someone more experienced in this department of surgery, and perhaps under the guidance of expert advice carry on the details of treatment.

The following pages are an attempt to simplify the rather complex subject of genito-urinary disease, to describe in detail the diagnostic and therapeutic measures accessible to every medical man, and to state definitely the boundary at which the province of general medicine ends and that of urology begins.

\section{TECHNIC}

In the manipulation which constitutes so large a part of genito-urinary therapeusis gentleness is all essential. The intact mucous membrane of bladder and urethra is really very 
resistant to infection. This fact is strikingly proved by the excellent condition which the bladder mucosa may show in spite of a stream of pus constantly pouring over it from a damaged kidney. If the mucous membrane be torn or abraded, however, infection by the bacteria which are necessarily introduced or which are already present will take place and set up an inflammatory reaction. Usually mild, this reaction may be enough to cause peri-urethral abscess, prostatitis, and even septicemia. Careful attention to asepsis is necessary, but even more important is absolute avoidance of trauma to the lining of the urinary tract.

Care in manipulation, therefore, is the first consideration in the avoidance of infection. There is another rule which must always be observed; that is, instrumentation must be followed by lavage. If the posterior urethra has been entered, the lavage must include the bladder; if only the anterior urethra, an anterior wash will suffice. If the bladder is to be included, 8 ounces of the solution are left in that viscus and are voided after the catheter is removed. The solution used consists of one of the mild antiseptics, such as boric acid 2 per cent., potassium permanganate $1: 3000$ to I : I0,000, or silver nitrate I : 5000 or weaker.

The antiseptic solution, flowing with considerable force over the mucous membrane of the urethra, carries out with it bacteria which may have been deposited along the canal. -The strict observance of this rule is imperative.

The value of urinary antiseptics in the prevention of infection from instrumentation I believe to be greatly exaggerated. 
That they are of the greatest importance in certain.conditions of the genito-urinary tract goes without saying; used as a prophylactic measure they certainly do no harm. The administration of one of them previous to instrumentation of the lower urinary tract is a safe precaution, but is not essential, provided instrumentation is properly done.

Asepsis in Urethral Instrumentation.-In urethral instrumentation there are certain minimum requirements of asepsis which must be observed. The hands should be carefully washed with soap and hot running water, and dried with a clean towel. A sterile catheter lubricant, preferably one of the sea-moss preparations put up in collapsible tubes, is dropped upon the tip of the instrument. The hands, even though washed, are not sterile, and should not touch that part of the instrument which will enter the posterior urethra -that is, they should not come in contact with the instrument within 3 or 4 inches of the tip.

The instrument itself may be sterilized in one of several ways; the cleansing process should start at the time the instrument was last employed. Immediately after use, before the adherent mucus dries, all metal and rubber instruments should be washed in hot running water, boiled for at least two minutes, dried with a clean towel, and put away in a closed drawer or case until used. If their container is reasonably dust-proof and the instruments are employed frequently they may be used without further sterilization. If there is doubt of their cleanliness, they should be again boiled before being used. Complicated instruments, such as 
the Kollmann dilator and those with electrically lighted bulbs attached, should not be boiled, but can be cleaned by washing with lukewarm water and soap, followed by alcohol. They may then be further sterilized by formalin gas.

The apparatus generally used for this purpose consists of a metal box long enough to hold sounds and catheters, with a tightly fitting lid. At one end is a shelf; upon this a pastil of formalin is placed. The compartment beneath this shelf has a separate opening at the end of the box, and contains a small alcohol lamp. The heat of the flame liberates formaldehyd from the pastil, and fills the sterilizer with the gas. The instruments are left in this for twelve hours or more.

Catheters and bougies made of woven fabric coated with varnish should be washed with soap and water, dried and sterilized with formaldehyd gas; some kinds, such as those made by certain French firms, may be boiled. They must not be allowed to cool while in contact with other instruments or with the sides of the sterilizer, for the varnish, softened by heat, will lose its smoothness if touched.

Finger-cots for rectal examination should be boiled after each case. The infectious organisms to be especially guarded against in genito-urinary practice are the gonococcus and the Spirochætæ pallida. Both organisms are easily killed by heat; the gonococcus is said to be killed by exposure to a temperature of $120^{\circ} \mathrm{F}$. Immersion of instruments in boiling water for two minutes is sufficient therefore to insure against their carrying these micro-organisms from one patient to another. 
Equipment.-For the practice of genito-urinary surgery there are certain things which are practically essential and others which are not essential, but which are very useful. In the following list will be given the essential equipment. In a supplementary list will be given the equipment which is desirable, but not necessary.

\section{NECESSARY EQUIPMENT}

\section{General:}

Hot and cold running water.

Sterilizer for boiling instruments. (At least 12 inches long.)

Examining table. ( 32 or 34 inches high.)

Microscope, slides, and cover-glasses.

Irrigating reservoir, glass, capacity at least 24 ounces, with rubber tube, glass nozzle, and cut-off.

Glass graduate or enamel pitcher (capacity I6 ounces).

Stirring rod, glass pipet.

Enamel basin.

Urinometer. Two conical urine glasses.

Finger-cots.

\section{Instruments:}

Soft-rubber catheters about Nos. 14, 16, or 18 French.

Woven bougie catheters, sizes I2, I4, and I6 French.

Woven coudé catheter, size I4 French.

Stylet of substantial character and a soft-rubber catheter to fit it.

Bougies, filiform and alternate sizes from Nos. 6 to 20 French. 
Bougie catheter with filiform which screws to its tip.

Metal sounds, sizes 20 to 32 .

Metal or woven bougies à boule, sizes 16, 20, 24, and 28 .

Drugs:

Catheter lubricant. (The kind made of sea-moss and dispensed in collapsible tubes is very convenient.)

Acetic acid, 33 per cent.

Potassium permanganate (3-grain tablets. One in 16 ounces makes a $\mathrm{I}: 2500$ solution).

Silver nitrate (tablets, I grain each. One in 8 ounces makes a $I: 4000$ solution).

Boric acid crystals (saturated solution equals 4 per cent.).

Nitric acid (concentrated).

Fehling's solutions A and B.

Oxalic acid crystals (for removing stains of potassium permanganate).

Alcohol, 95 per cent.

Litmus paper.

Stains:

Löffler's methylene-blue.

Aniline oil gentian violet.

Gram's solution (IKI).

Bismarck brown.

\section{Equipment Desirable, But Not Necessary}

Large syringe for bladder irrigation. (The all metal and the glass and metal kinds are the best.)

Kollmann dilator. 


\section{AN OUTLINE OF GENITO-URINARY SURGERY}

Keyes' instillator.

Centrifuge.

Formaldehyd sterilizer.

Various catheters with prostatic curves and tips.

Straight electrically lighted endoscope.

\section{ENDOSCOPY AND CYSTOSCOPY}

Endoscopy.-Actual inspection of the urethra, anterior and posterior, is a procedure which at times yields information of much value. According to some writers it should be performed in every case of persistent urethral discharge. It is their contention that by means of the urethroscope one will detect many lesions otherwise overlooked, and that by applications and manipulations through the urethroscope these lesions can be cured.

Views such as these, which may be found in many textbooks and articles upon the management of urethritis, seem to me to place far too much emphasis upon the operation of endoscopy. In eight years' experience in clinic and private practice I have seen only a few cases in which endoscopy contributed information of any importance. The ruling out of a definite lesion is sometimes helpful, and in this direction lies the chief value of endoscopy.

Papillomata or condylomata of the urethra can be accurately diagnosed by this measure, and can be treated only by fulguration or excision through the endoscope. Small granulating areas of the urethra will heal up as quickly when treated by dilatation and massage of the urethra upon a 
sound as when touched with caustic. Lesions of the mucosa of the posterior urethra are, as a rule, due to infection of the prostate or vesicles. Treatment of the surface alone will not suffice-the underlying cause must be removed by prostatic or vesicular massage.

The discovery of congestion of the verumontanum in a case of sexual or urinary disturbance does not materially aid in relieving the symptoms, and in my experience the application of silver nitrate to the posterior urethra is more successful when made with the Keyes' instillator than when applied by a swab through the endoscope.

In the management of diseases of the female urethra endoscopy is really essential, and should be used much more frequently than at present. There are many cases of socalled "cystitis" in which the lesion is not in the bladder, but in the urethra. The most effective treatment is by means of applications through the urethroscope.

The technic, both in male and female, is not difficult. The simplest instruments are the best. A straight, electrically lighted endoscope, consisting of sheath, obturator, and a lamp carrier which passes through a small channel separate from the main lumen, is the most generally useful. It can be used in making applications both to the anterior and to the posterior urethra. For inspection of the posterior urethra dilation of the canal by water gives a much better picture, and can be carried out best by means of a cysto-urethroscope or close-vision cystoscope.

For endoscopy of the anterior urethra no anesthesia is 
necessary. For manipulations in the posterior urethra local anesthesia secured by the instillation of 2 or 3 c.c. of 4 per cent. cocain solution is desirable.

Anterior Urethroscopy. - The penis is drawn out so as to eradicate folds in the urethra. The urethroscope is passed as far as the triangular ligament (cut-off muscle), the obturator is removed, and the instrument is slowly withdrawn by the right hand, while the left hand keeps the penis taut. The mucosa appears reddish pink, with longitudinal striæ of deeper red. Granulating areas appear as dull red spots which bleed easily. Papillomata and condylomata are easily identified. The incision of the orifices of peri-urethral glands is mentioned by some writers as a possibility, but is a procedure which can be carried out only by operators of unusual deftness.

Endoscopy in the female is best done with the patient in the knee-chest position. The bladder must be empty. The endoscope is passed into the bladder; the obturator is removed and the instrument withdrawn until the diaphragm of the sphincter closes over the tip. The walls of the urethra are inspected and, if necessary, applications are made with a cotton-tipped probe as the endoscope is withdrawn.

Posterior Urethroscopy.-When the cysto-urethroscope is used the instrument is passed into the bladder and then withdrawn so that the window is between the sphincters. A stream of boric acid solution (2 per cent.) is gently injected by an assistant or by means of an irrigator. The fluid distends the urethra and enables one to inspect the walls and the veru- 
montanum. Between the internal sphincter and the verumontanum is a depression. The floor of this is frequently streaked with red and naay show ribs of mucous membrane. Then the verumontanum rises abruptly and fills the field of vision. Its surface should be smooth and pink; when pathological it may be congested, cystic, or papillomatous. The anterior aspect of the verumontanum contains the opening of the utricle, which, if infected, may exude pus. In this connection it is easy to let the imagination run away with one's surgical common sense. Such procedures as washing out the utricle and catheterizing the ejaculatory ducts sound very effective, but unless one is an expert cystoscopist he should not attempt them. The case of gonorrhea that cannot be cured by the simple measures of dilatation, lavage, and massage is a rarity. The chances of doing harm by more complicated treatment are many. In making applications to the verumontanum either the straight endoscope or the posterior urethroscope, with window on the side, may be used. After the field is wiped dry it is swabbed with silver nitrate solution in strength varying from $\mathrm{I}$ to ro per cent. Too strong solutions will excite too great reaction.

Cystoscopy.-The value of cystoscopy admits of no argument. Without it diagnosis of genito-urinary disease is in many cases impossible. Today the practice of renal surgery without preliminary study of each kidney by itself amounts to malpractice.

As to the advisability of the man in general practice attempting to do cystoscopy there is some difference of opinion. 
The actual performance of cystoscopy is not difficult. The difficulty lies in interpreting what one sees. It stands to reason that the opinion of a man who does only ten or twelve cystoscopies a year cannot be as reliable as that of the man who does several hundred. 'It would seem better, therefore, for each district to support one cystoscopist, whose opinion, by reason of his experience, would become of very definite value.

Technic.-In our experience the Brown-Buerger cystoscope has proved to be the most generally useful. It consists of a sheath bearing upon its tip a hooded electric light, an obturator which closes the window in the sheath during its introduction, and observation and catheterizing telescopes. These latter contain the system of lenses which enable one to inspect a surface which lies parallel to the long axis of the instrument. The principle is the same as that of the now famous periscope. The catheterizing telescope is smaller in diameter and has two grooves for ureteral catheters, and a lever with which the tips of the catheters are directed into the ureters. Two types of sheath are furnished with each instrument. One has the window upon the convex side, the other has it upon the concave side. The latter is better for observation alone; the former is used especially in catheterizing ureters in a contracted bladder.

Before passing the cystoscope the urethra should be anesthetized by the instillation of 4 per cent. solution of cocain. In most cases this will suffice; in very irritable bladders, such as the contracted, ulcerated bladder of tuberculosis, a more 
extensive anesthesia is necessary. For this purpose spinal anesthesia is ideal. There is no spasm of the bladder, and renal secretion does not diminish as it does when the patient is etherized. Before introducing the cystoscope always test the light. If that is satisfactory, pass the cystoscope into the bladder. Sometimes meatotomy is necessary to allow of the passage of the instrument, the caliber of which is 22 or 24 French. When the tip is in the bladder the obturator is removed and the bladder is washed with boric acid solution through the hollow sheath until the water returns clear. If there is persistent bleeding the addition of adrenalin to the solution will be of great assistance. The observation or catheterizing telescope is then introduced and the bladder filled with solution through a tube attached to one of the side cocks. The light switch is then turned on and inspection begins.

A routine method of inspection should be followed. First the roof is examined, then each lateral wall, and finally the base. The condition of the bladder musculature-whether trabeculated or not-the presence of diverticula, stones or tumors, should be noted. The color and quality of the mucous membrane-whether pale and shining, and marked by fine capillaries, or whether light-absorbing and of a uniformly reddish color-are important. The character of the ureteral jets and of the ureteral orifices should be studied next. Lastly, the condition of the bladder neck is examined. If the patient is a man, the prostatic outline should be mapped out. In cases with obstruction at the bladder orifice the 
presence of the anterior cleft, denoting enlargement of the lateral lobes, or the elevation of the posterior lip by the middle lobe or bar, is especially important.

The search for the ureters is commenced by passing the cystoscope as far in as it will go and locating the bubble on the roof of the bladder. The instrument is then rotated half a circle, so that the window looks directly downward. The instrument is then withdrawn until one notices that the lax, wrinkled mucosa of the base has changed into a smooth, white, elevated area. This is the trigone. By following the upper line of the trigone to either side, the eye is carried on to the ureteric ridges.which run obliquely upward away from the observer. Upon the crest of each ridge a short distance above its junction with the trigone lies the ureteral orifice. In certain instances the orifices are visible only when they open to emit urine.

In bladders distorted by long-standing infection, especially that of tuberculosis, the ureters may be impossible to find unless the urinary jet is artificially colored. This is best done by the intravenous injection of 5 c.c. of 4 per cent. solution of indigocarmin in water, which has been sterilized by boiling. Within. five minutes after the injection the jet from a normal kidney should be colored a deep blue and will make easy the location of the ureteral orifice. The relative intensity of color of the jets from the two ureters will give an idea as to the relative functions of the kidneys.

The passage of the ureteral catheter is a matter of 
technic which is readily learned by one who has a chance for frequent practice. Occasionally the ureteral orifice is easily visible, but is hard to catheterize. It is helpful to change the amount of fluid in the bladder. Further distention will flatten the base and may make catheterization a great deal easier.

Cystoscopy in Children.-Boys of ten or more and girls of six may be cystoscoped if an instrument of 16 or 18 French is used. Such a cystoscope is made on the Brown-Buerger style, but with only one catheterizing channel. General anesthesia is necessary.

Operative Cystoscopy.-Elaborate operating cystoscopes have been invented. Hugh Young's model has a pair of jaws which can be used to pick up small stones or foreign bodies, or to nip off small tumors. Leo Buerger has devised several instruments which may be workel through his operating cystoscope. There are scissors, a pair of nippers, and a dilator. These are intended chiefly for aiding the passage of stones low in the ureter. With the scissors the ureteral orifice may be slit, with the dilator it may be stretched. The nippers can be used to grasp the stone if it is in sight, or to bite out a bit of mucous membrane for diagnosis. Hugh Young has devised ingenious appliances which can be operated through the cystoscope for applying radium to bladder tumors.

These intravesical manipulations should be left to the expert cystoscopist. They are applicable only in exceptional cases, and their usefulness is distinctly limited. 


\section{AN OUTLINE OF GENITO-URINARY SURGERY}

CABOT, H.: The Training of the Urologist, New York Med. Jour., May 25, 1912.

Schmid, L. E.: Why Úrology Should be Considered a Specialty, Trans. Amer. Urological Assoc., 1912, vi, 1-12.

TномаS, B. A.: The Significance of Specialism with Reference Especially to Genito-urinary Surgery, Jour. of Penn. State Med. Assoc., 1916, $\mathrm{xx}, 101$. 


\section{CHAPTER II}

\section{URINARY ANTISEPTICS}

URINARY antiseptics are those drugs which, taken by mouth, are followed by the excretion through the kidnevs of a substance hostile to the growth of bacteria. Experience with various drugs has resulted in the elimination of all but two-i.e., the balsamics and hexamethylenamin. Different preparations of these two are employed, but the value of each depends upon the action peculiar to its parent drug. These two drugs act in widely different ways.

The Balsamics.-These drugs (sandalwood oil, oil of copaiba, and oil of cubebs) are excreted partly by the lungs, but chiefly by the kidneys. Cushny (Pharmacology, p. 75) says: "The products of the oils excreted in the urine appear to have some antiseptic action, for the urine of persons treated with them putrefies more slowly than ordinary urine and the growth of many of the more common germs is somewhat retarded by it. On the other hand, there seems some question as to how far it is destructive to the gonococcus, which sometimes grows readily in culture-media made up with such urine instead of water. Winternitz therefore attributes the undoubted therapeutic efficacy of these oils to their lessening the inflammatory exudate rather than to their antiseptic ac- 
tion, without denying that the latter may also be of some importance."

With these conclusions practical experience closely agrees. Of the balsamics, sandalwood oil is best borne by the stomach, is less likely to produce skin eruption, and is as efficacious as any of the others.

The chief use of sandalwood oil is to allay inflammation of the mucous membrane of the lower urinary tract. In the acute stage of gonococcus infections, in acute cystitis of any origin, and especially in cystitis due to the tubercle bacillus, sandalwood oil best demonstrates its value. Frequency and burning on urination diminish. It is given usually in capsules (ro minims) after or during each meal. In certain individuals this drug will cause hyperacidity and gastric distress, in others its use is followed by severe colicky pain in the kidneys. In these cases the drug had better be discontinued.

With the synthetic products (gonosan, arrhovin, santyl, etc.) we have had no experience. Keyes (p. 2I3, I9II) considers them no more and no less potent than the more familiar balsamics.

Hexamethylenamin of the United States Pharmacopœia is practically the same as urotropin, which is a patented German product. It dissolves rather slowly in water, and should be taken after meals in order to avoid any irritant effect which it might have upon the empty stomach. The drug is inert and has no bactericidal effect until it is broken up into ammonia and formalin, a process which requires an acid 
medium. To the formalin thus set free the drug owes its value as a urinary antiseptic.

In order to get any benefit from its administration the urine must be definitely acid. With an acid urine the process of splitting off formalin begins in the kidney and is continued in the bladder. The value of hexamethylenamin in the treatment of kidney infections has been doubted, on the ground that the drug does not stay there long enough for any important amount of formalin to be set free. In distinctly acid urine warmed to body temperature enough formalin may be liberated in three minutes to give a definitely positive test (Burnam's test). On theoretical grounds it would seem easily possible for urine to remain this length of time in the kidney, if we include its passage through the various tubules as well as its diffusion through the pelvis. As a matter of practical experience there is ample evidence to show that the administration of hexamethylenamin influences very markedly certain types of renal infection. As the liberation of formalin continues in the bladder, one can easily understand its value in keeping down infection within that viscus.

The greatest value of hexamethylenamin is in combating colon bacillus infections of the urinary tract. The urine is naturally acid in this condition, thereby assisting in the liberation of formalin, and the colon bacillus is very susceptible to a constant bath of formalin. The coccus infections are less responsive to this type of therapy. In acute inflammatory conditions of the lower urinary tract and in infections by the tubercle bacillus hexamethylenamin is contraindicated. The 
sensitive mucosa is irritated by the formalin in the urine, and the local hyperemia and discomfort are increased.

In order to get the best effect from hexamethylenamin it must be administered with an understanding of its behavior. The urine must be acid and not too dilute. If not definitely. acid to litmus paper it may be made more so by the administration of sodium acid phosphate so to 20 grains three times a day. As monosodium phosphate and disodium phosphate are both constituents of normal urine, the administration of the monosodium phosphate (sodium acid phosphate) does not introduce a foreign substance, but simply swings the balance on to the acid side. Sodium acid phosphate is now supplied in Io-grain tablets, and may be employed to the point of causing diarrhea.

The amount of hexamethylenamin to be given depends upon the extent to which it is broken up. Some persons taking 22 grains a day will liberate enough formalin to give the symptoms of bladder irritation; 15 grains a day is too small a dose for the average adult. As formalin appears in the urine for about eight hours after its ingestion, the administration of ${ }_{5}$ grains three times a day has proved to be a proper dosage for most individuals. The drug should be increased to a point where it either controls the infection or gives signs of irritation. As the liberation of formalin continues after the urine leaves the kidney, it seems fair to say that one should not expect renal irritation to occur until after bladder irritation has set in; the first signs of an overdose would, therefore, be vesical. 
The presence of formalin in the urine may be demonstrated by Burnam's method. "This test consists of adding to the suspected fluid 3 drops of 0.5 per cent. aqueous solution of phenylhydrazin hydrochlorid and then 3 drops of a 5 per cent. aqueous solution of sodium nitroprussid, then an excess of saturated aqueous solution of sodium hydroxid. It is important that the solution to be tested, as well as the sodium hydroxid, be slightly warmed, a little more than body temperature. When formaldehyd is present in solutions of $\mathrm{I}$ : 20,000 or stronger there follows an intense blue color, which gradually changes to green, and then after a few minutes to brown. In solutions of less than I :20,000 the first color is the intense green, which passes off into brown. The test is delicate down to 1 : 150,000 or less. When a solution is tested and found to be negative, as is the case when urotropin is added, it can be acidulated with sulphuric acid, heated to boiling, cooled off and tested, when the reaction will be positive, due to the breakdown of urotropin into formalin."

Although this test has proved valuable in establishing certain facts in regard to the excretion of formalin, it is no longer a necessity. It has been shown ${ }^{1}$ that hexamethylenamin is always excreted promptly by the kidney except in cases of advanced interstitial nephritis; that the hexamethylenamin in the urine is broken up into formalin in proportion to the acidity of the urine. In the administration of hexamethyl-

${ }^{1}$ Smith, G. C.: The Excretion of Formalin in the Urine; An Inquiry into the Accuracy of Burnam's Test, Boston Med. and Surg. Jour., 1913, clxviii, 713-716. 
38 AN OUTLINE OF GENITO-URINARY SURGERY

enamin, therefore, one has only to make sure that the urine is acid; the production of formalin will then be assured.

Other Drugs.-Sodium benzoate and salol have been used for urinary infections, but in our experience they have accomplished very little.

Hinman, F.: An Experimental Study of the Antiseptic Value of the Internal Use of Hexamethylenamin, with the Report of a Simple Clinical Method of Quantitative Estimation of Formalin, Trans. Amer. Assoc. Genito-urin. Surg., 1913, viii, 235-269. 


\section{CHAPTER III}

\section{URINALYSIS. TESTS OF RENAL FUNCTION}

URINALYSIS

IN nine-tenths of all genito-urinary cases the condition of the urine gives to the careful observer information of great value. In obtaining a specimen for the ordinary examination it is enough in the male simply to have the foreskin retracted, so that the urine is not contaminated by subpreputial detritus. In the female, however, a catheter specimen must be obtained; unless this is done, one cannot be sure that the abnormal cells which may be found are not from the vagina. I do not remember having seen bladder infection result from this procedure. One should always observe the precaution of filling the bladder with an antiseptic after catheterization.

The general characteristics of the urine, which should be examined soon after it is passed, must be noted. These are:

Odor-ammoniacal or not?

Color-pale or concentrated; bloody or smoky?

Opacity-clear and sparkling; hazy; turbid?

Contents-shreds; clots; bits of tissue?

Reaction to litmus paper?

Specific gravity?

Presence of albumin; sugar?

Sediment (microscopic examination)? 
Albumin.-If the urine when passed is hazy, a few drops of acetic acid may be added to a small portion. If the opacity disappears, it is due to phosphates. The urine thus cleared is tested for albumin by nearly filling a test-tube with it, and boiling for a few seconds the urine near the top of the tube. By holding the tube against a dark background even a very slight cloud can be detected. If this cloud does not clear upon the addition of a few drops of acetic acid it is due to albumin. If a heavy cloud is present, or if the urine is cloudy with some substance other than phosphates, the amount of albumin present may be estimated by the nitric acid test (ro c.c. urine filtered, in wine glass; underlay this with concentrated nitric acid, either by decanting or by introducing the nitric acid with a pipet). The thickness and density of the white ring at the junction of the urine and the acid indicate the amount of albumin present.

Sugar.-Boil 5 c.c. Fehling's alkaline solution with 5 c.c. Fehling's copper sulphate solution; add a few cubic centimeters of urine and boil. Sugar is indicated by the formation of a yellow precipitate. If the test is positive, it must be repeated without boiling after the addition of the urine, as occasionally other substances besides sugar will cause a precipitate when boiled with Fehling's solution.

Sediment.-The principal objects to be looked for are puscells, red blood-cells, and casts. Other cells, if present in quantities, may have some significance, such as the caudate cells of the renal pelvis. In fresh urine the presence of more than a few bacteria is noteworthy. 
Bacteria are best shown in stained sediment. To obtain the sediment for this the urine is decanted out of the centrifuge tube (after sedimentation) and the sediment is picked up in a pipet or platinum loop, put on a clean slide, dried and stained with Löffler's methylene-blue. Urines containing pus and no bacteria in a sediment so tested suggest strongly tuberculosis of the genito-urinary tract. As the great majority of infections are due to the colon bacillus or to cocci the type of infection can often be quickly discovered.

Confirmation can be obtained by the use of cultures, but it is a fact that, not infrequently, stained sediments tell the truer tale, as in cultures the change in medium may be advantageous to the growth of some kinds of bacteria-and detrimental to that of others.

If tuberculosis is suspected, 20 minims of the sediment may be injected into the peritoneal cavity of a guinea-pig. After five or six weeks the pig is killed and liver and spleen examined microscopically for tubercles. Search for the tubercle bacillus in the urine is likely to be misleading unless the urine is obtained by catheter. Otherwise the smegma bacillus, which is an acid-fast organism, may appear and simulate the tubercle bacillus. Unless tubercle bacilli are very numerous they will not be found in the ordinary sediment. The following routine has proved fairly satisfactory in detecting the tubercle bacillus in a considerable proportion of cases.

Specimens Stained for the Tubercle Bacillus.-A specimen of the suspected urine is centrifuged at high speed for a considerable time (fifteen minutes to one hour). The sediment 
is spread thin upon a slide, dried, fixed by being passed through the flame, covered with carbolfuchsin (Ziehl's solution), and heated to the point of steaming.

The carbolfuchsin is washed off with Czaplewski's reagent or with 20 per cent. nitric acid, followed by 95 per cent. alcohol. This decolorizing process is continued until the smear shows only a faint pinkish color. It is then washed in water and counterstained with Löffler's methylene-blue. The tubercle bacilli appear as bright red rods in a blue field (see Plate I).

Urethral and Prostatic Secretion.-Smears from urethra (Gram's stain): A loopful of discharge from within the meatus is spread thinly upon a slide, dried, and fixed by being passed through the flame twice. The smear is covered with aniline oil gentian violet (Stirling's) and heated until it just begins to steam; allowed to remain thirty seconds, and then replaced, without washing, by Lugol's solution (IKI). This remains for thirty seconds. The slide is then washed with 95 per cent. alcohol until the violet color has almost disappeared. It is then washed in cool water for a few seconds and covered with the counterstain (Bismarck brown or eosin) for one minute.

Bacteria, to be called gonococci, must occur within the protoplasm of the leukocytes, must be arranged in rectangular figures, and must be brown or red, rather small, and biscuit-shaped diplococci (see Plate I).

The secretion from the prostate and vesicles in chronic cases (which are the ones in which the secretion is usually 


\section{PLATE 1}

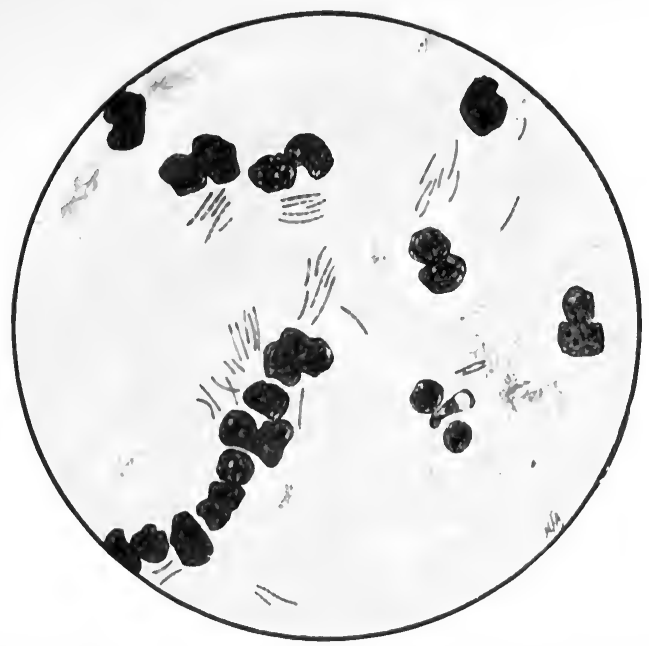

Tubercle bacilli in urinary sediment. Stained with carbol-fuchšin, decolorized, and counterstained with methylene-blue. Specimen was drawn by ureter catheter from a tuberculous kidney which, after removal, showed very marked involvement of the pelvic mucous membrane.

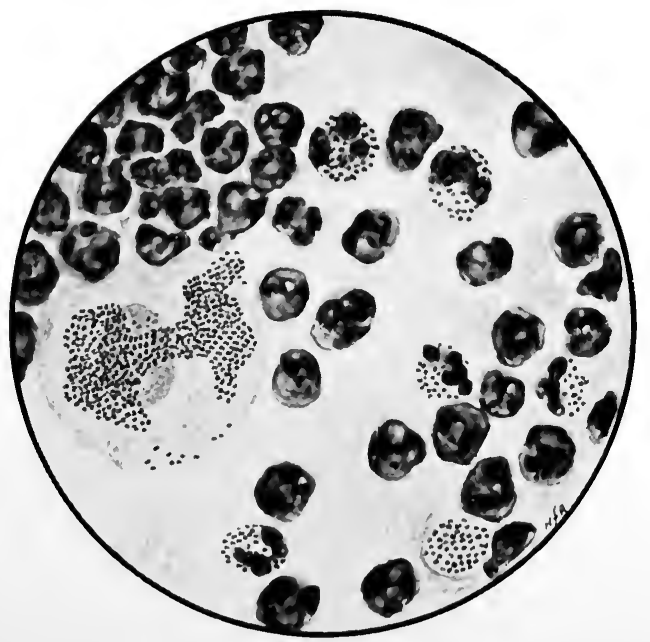

Secretion from the urethra in acute gonorrhea stained by Gram's method. Gonococci appear in several of the lcukocytes and in the large epithelial cell. 

PLATE 2

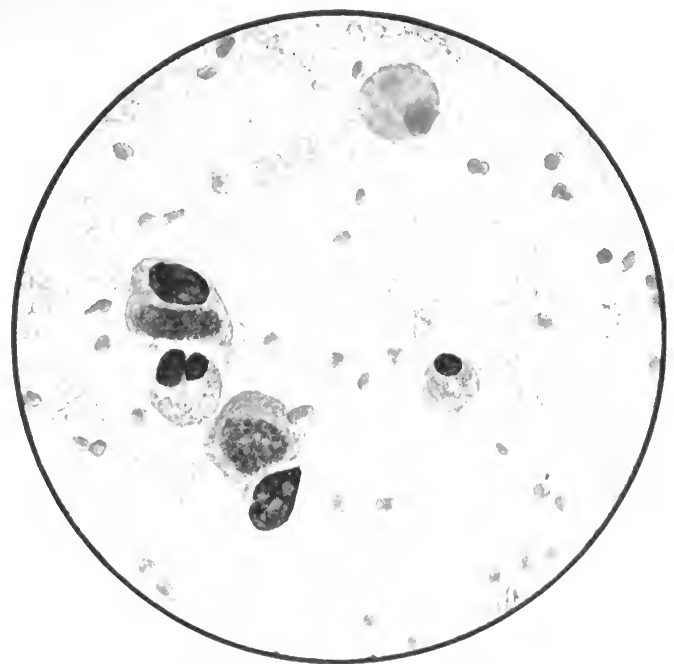

Secretion from normal prostate and vesicles. The larger cells are epithelial, the small oval bodies are the heads of the spermatozoi.

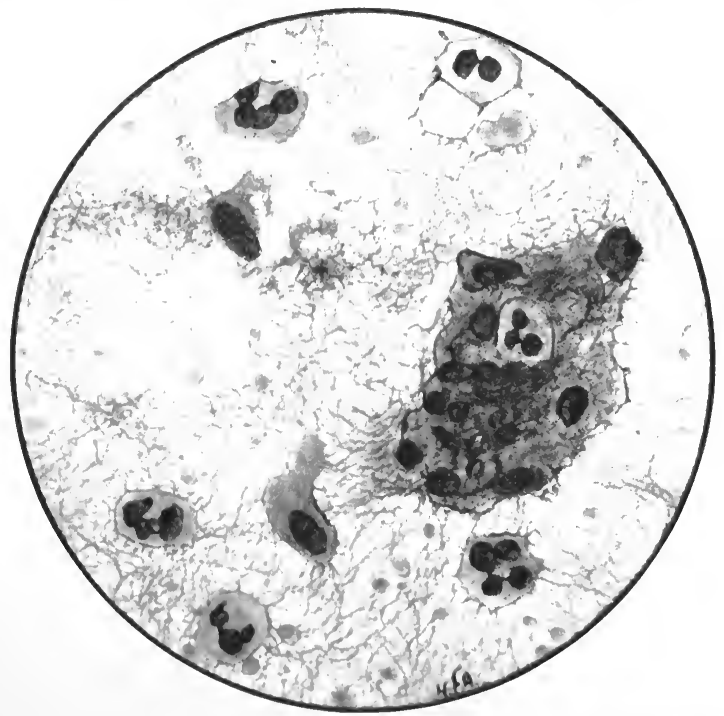

Secretion expressed by massage from prostate and vesicles infected by the gonococcus. The polymorphonuclear leukocytes indicate infection. The larger mononuclear cells are from the epithelium. The small, oval, homogeneous staining bodies are tle heads of spermatozoa. 

examined) contains gonococci in such small numbers that I personally have given up trying to find them. Other organisms get a foothold, and among them it is difficult to find the gonococcus. Even if the search gives negative results, one cannot be sure that the infection is cured. The chief value of such a smear is to show whether or not there is pus, and in what amounts, and whether or not spermatozoa are present. For this purpose a methylene-blue stain will do perfectly well. The smear should be studied under the oil immersion. The normal prostatic secretion shows a homogeneous blue background dotted with the heads of spermatozoa, stained a darker blue; large mononuclear epithelial cells may occur in large numbers, and occasionally a few pus-cells. More than an occasional polymorphonuclear leukocyte is, to my mind, abnormal (see Plate 2).

Gonococcus Complement-fixation Test.-This test is based upon the presence in the blood of specific antibodies. These are supposed to occur in the blood of individuals who harbor the gonococcus; they are the response of the organism to its invasion by the gonococcus. If the invasion is slight, as in simple urethritis, this reaction may not be aroused. In any case the antibodies are not likely to be found until three or four weeks after the beginning of the infection.

The test is carried out according to the same principles as the Wassermann test. About 2 c.c. of blood are drawn from the median basilic or cephalic vein. The serum is inactivated by heat to destroy its content of complement, and a definite quantity is mixed with a known quantity of complement 


\section{AN OUTLINE OF GENITO-URINARY SURGERY}

(guinea-pig's serum) and antigen (emulsion of gonococci). This mixture is incubated, and if the specific antibodies are present they will utilize all the complement in their union with the gonococci. Amboceptor (sensicized sheep. serum) and washed red blood-corpuscles are then added, and the mixture is again incubated. If the complement has been utilized by the union of antibody with gonococci, the sheep serum (amboceptor) cannot hemolyze the corpuscles, and they remain intact (positive test). If, on the contrary, the suspected serum has no antibodies, complement will be left free to unite amboceptor and red blood-cells, and hemolysis of the latter will occur (negative test). The reaction is serum + antigen \pm complement + amboceptor + sheep corpuscles. Various degrees of hemolysis occur; in our experience, a weakly positive test may be disregarded as being due perhaps to a socalled "group" reaction.

It stands to reason that as complicated a test as this can be of value only when done by an experienced pathologist. Even then it should be regarded simply as one of the data which aids in the formation of an opinion as to diagnosis or question of cure.

\section{RENAL FUNCTION TESTS}

Estimation of renal function, to be accurate, should be based upon the clinical history, the digestive symptoms, the blood-pressure, the amount of non-protein nitrogen in the blood, and the general condition of the patient. The kidneys themselves are the last organs to be examined. 
In coming to a correct conclusion as regards the renal condition of a patient the functional test devised by Rowntree and Geraghty is of great assistance. It seems to be the most practical of any of the artificial tests, and indicates with surprising accuracy the changes in the renal function which in pathological cases occur from time to time. It must be remembered that the output of phenolsulphonephthalein (upon which the test is based) is affected by severe changes in the general condition of the patient. Anesthetization, shock, severe sepsis, debility - all these influence the amount of dye which is excreted. The test registers the functional value of the kidneys at the time of the test, and may be quite different a week later. In this sensitiveness resides a good part of its value. The drug is thought to be excreted by the epithelium of the tubules of the kidney, and acute infectious processes in the kidney, which have caused an edema of the renal tissue, reduce the excretion to a marked degree. As the infection subsides the test registers the corresponding improvement in renal function.

Technic.-A solution of phenolsulphonephthalein containing $6 \mathrm{mgm}$. to the cubic centimeter may be purchased in ampules (Hynson and Westcott). Exactly I c.c. is injected into the muscles of the back or thigh. A catheter is passed and the urine allowed to drip in to a test-tube containing about I c.c. of 25 per cent. $\mathrm{NaOH}$. When the phthalein begins to come through, which normally occurs in seven minutes, a pink color appears in the alkaline solution. The catheter is then withdrawn; at the end of one hour the patient urinates 


\section{6 \\ AN OUTLINE OF GENITO-URINARY SURGERY}

or is catheterized. The urine excreted during that hour (since the first appearance of the color) is made strongly alkaline by the addition of sodium hydroxid, and diluted with tapwater up to I liter. A specimen from this solution is then compared with solutions containing known quantities of the dye, to determine what percentage of the amount injected has been excreted. Normal kidney output is 45 per cent. (from 40-50 per cent.) in one hour. The estimation may be carried out in several ways. Hynson and Westcott have put out, for $\$ 5.00$, a square box about 4 inches in its greatest dimension, containing a little bottle for the solution to be tested, and thirteen bottles containing graded strengths of the dye. By direct comparison one arrives at the percentage excreted. ${ }^{1}$ A similar apparatus for temporary use can be made by diluting I c.c. of the phthalein solution to varying degrees, and comparing these dilutions with the sample to be tested. Test-tubes of the same diameter should be used; the solutions fade unless they are strongly alkaline and in sealed tubes. ${ }^{2}$

In case the urine contains enough blood to obscure the

${ }^{1}$ As these solutions may fade, their strength should be checked up occasionally by comraring them with a fresh solution.

${ }^{2} \mathrm{As}$ each ampule contains more than 1 c.c., 0.2 c.c. of the excess may be added to 200 c.c. of water, thus making a 100 per cent. solution. In estimating the amount excreted a specimen of the urine to be tested, already diluted to 1000 c.c., is compared with a known quantity of this 100 per cent. solution. Water is added to the latter until the colors match. The total amount : the original amount as $100:$ the desired percentage. For example: 2 c.c. of 100 per cent. solution is diluted to 8 c.c. before the proper color is obtained. $8: 2$ as $100: 25$. Therefore 25 per cent. is the percentage excreted. 
color of the phthalein, the blood may be coagulated by boiling and then filtered off.

The drug may be given intravenously. In that case normal appearance time is three minutes, and about 30 per cent. should be excreted in the first fifteen minutes after the color appears.

If one wishes to avoid catheterization the urine may be voided one hour and ten minutes after intramuscular injection; it should contain about 45 per cent. of the drug. If the output is normal, this method is sufficient. If below normal, one should know whether the appearance is prompt and the amount low, or whether the appearance is delayed. Unilateral renal disease might account for the first, but for the second, bilateral disease must exist.

More will be said of this test in later chapters. It is an aid in many conditions, and to the man in general practice will often supply information of considerable value.

Schwartz, H. J., and McNeil, A.: A Complement-fixation Test in the Diagnosis of Gonococcic Infections, Amer. Jour. Med. Sci., May, 1911. Further Experiences with the Complement-fixation Test in the Diagnosis of Gonococcic Infections, Trans. Amer. Assoc. Genitourin. Surg., 1912, vii, 89-108.

IRONS and NiCOLL: The Complement-fixation Test in the Iiagnosis of Gonococcal Infection, Jour. Infect. Dis., 1915, xvi, 303-310.

Uhle and Mackinvey: The Gonorrhea Complement-fixation Test, New York Med. Jour., 1915, cii, 737-739.

Krotoszyner, M.: Serodiagnosis of Gonorrhea, Calif. State Jour. Med., 1916, xiv, 451.

Sмiтh, L. D.: Complement-fixation Test in Diagnosis and Determination of Cure in Gonorrhea, Ill. Med. Jour., 1917, xxxi, 222.

Rowntree and Geraghty: An Experimental and Clinical Study of the Functional Activity of the Kidneys by Means of Phenolsulpho- 


\section{AN OUTLINE OF GENITO-URINARY SURGERY}

nephthalein, Jour. Pharm. and Exp. Therap., 1910, i, 579. The Phthalein Test, Archiv. Int. Med., 1912, ix, 284-338.

SANFord, H. L.: A Clinical Study of the Elimination of Phenolsulphonephthalein by the Kidneys, with a Report of 150 Cases, Cleveland Med. Jour., 1912, xi, 763.

Miller, R. H., and Савот, H.: The Effect of Anesthesia and Operation on the Kidney Function as Shown by the Phenolsulphonephthalein Test, Archiv. Int. Med., 1915, xv, 369-391.

BEER, E.: Kidney Function Tests. Interpretation with Reference to Significance of Minimal Excretion of Phthalein and Indigocarmin, Ann. Surg., October, 1916, lxiv, 434.

Christian, H. A.: Tests of Renal Function in Cases of Nephritis, Jour. Urology, 1916, i, 319.

Geraghty, J. T.: Kidney Function Tests, Southern Med. Jour., 1917, $\mathrm{x}, 194$. 


\section{CONGENITAL MALFORMATIONS}

Kidney and Ureter.-Many congenital malformations of the upper urinary tract give rise to no symptoms, and are discovered only at autopsy. Others, ordinarily because of interference with drainage, offer opportunities for infection, stone formation, and hydronephrosis. These are discovered in the course of investigation with cystoscope, $x$-ray, and pyelogram. Single kidney, horseshoe kidney, dystopic (misplaced or pelvic) kidney, double pelvis, double ureter, crossed ureter, and single ureter occur alone or in combination. Obviously, if they give no symptoms, they require no treatment; if they cause trouble, surgery may be necessary, but should be undertaken only after a thorough study of the urinary tract.

Horseshoe kidney may cause abdominal pain through pressure of its isthmus on the structures lying between it and the spine. In such an event the kidneys may be separated by division of the isthmus. This operation has been done, although not often. Either half of a horseshoe kidney may develop the same diseases as affect normal kidneys. Stone formation, tuberculosis, and pyonephrosis have occurred; if one-half of the kidney is sound the affected half may be removed. The technical difficulties may be considerable, but 
aside from that the diseased half of a horseshoe kidney should be treated as though the kidneys were separate.

Dystopic kidney is very liable to suffer from obstruction of its ureter. Infection supervenes, causing pyuria, fever, and pain. The picture is that of a tender mass low in the iliac fossa or in the pelvis. Usually such a kidney cannot be replaced with any prospect of success. Nephrectomy is almost always the best procedure.

Double ureter and double pelvis in themselves are not likely to cause trouble. Either half of a double pelvis kidney may become infected or tuberculous. Sometimes the diseased portion may be removed.

Bladder.-Diverticula of the bladder may be congenital or acquired. The latter are generally small, and are due to obstruction at the bladder outlet. Once this obstruction is removed, the diverticula cause very little trouble. The congenital type, on the other hand, is usually of good size when discovered and tends to constantly increase. It is single in the majority of cases ( 15 out of 19 in Thomas' series), and may be situated anywhere in the bladder, although the greater number occur at the base. Not infrequently the ureteral meatus is drawn into the diverticulum as it grows. Congenital diverticula are characterized by the absence of a muscular coat, and have a capacity varying from I ounce to 20 ounces or more. Cabot has suggested that they are due to the maldevelopment of ureteral buds.

Such a diverticulum prevents the bladder from emptying completely. The residual urine becomes infected; symptoms 
of cystitis appear which are more or less severe according to the virulence of the infection. 'The diverticulum may press upon the ureter, thereby causing renal retention and infec-

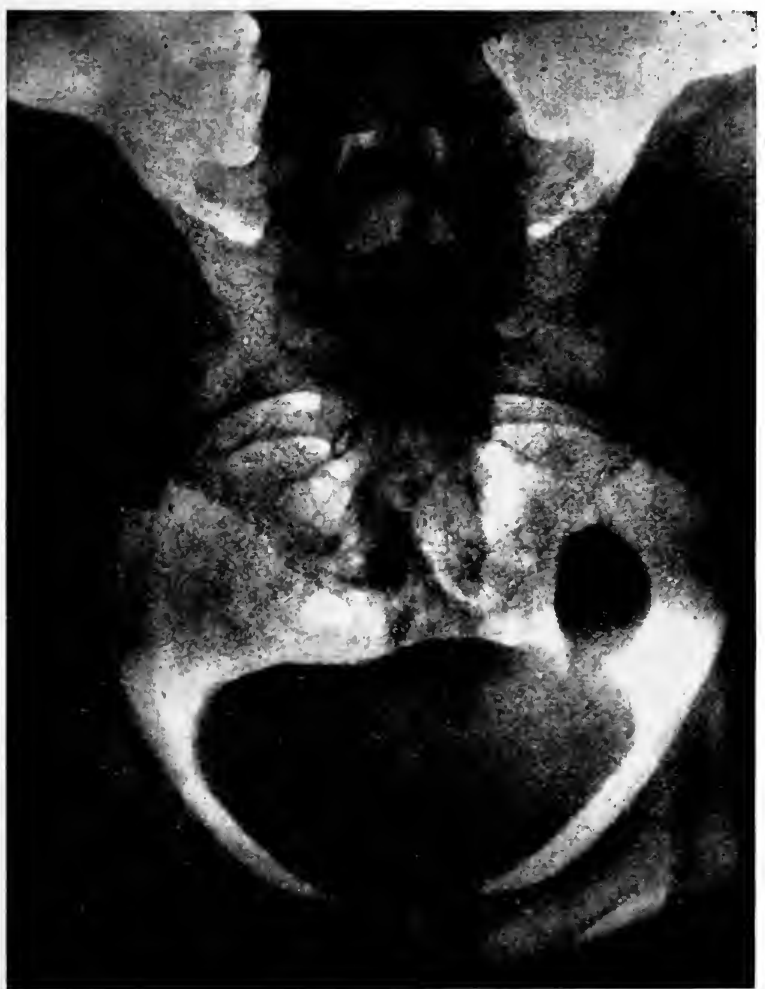

Fig. 1.-Bladder and diverticulum filled with argentide (Mass. General Hospital).

tion. Advanced cases present a picture similar to that of chronic prostatic obstruction-large residuum, urinary infection, renal insufficiency. Diverticulum should be suspected 
when these signs appear in aman under fifty years of age, and in older men into whose bladders a soft-rubber catheter passes very easily. Only a few cases in women have been reported (Lower). The diagnosis must be made between retention due to nerve lesions, that due to fibrous obstructing prostate (which may occur in men of forty or thereabouts), and that due to diverticulum. Radiographs of the bladder filled with an opaque fluid (collargol, argentide, or thorium) often show very distinctly the outline of diverticula (Fig. I). Exposures must be made at varying angles. Further study should be undertaken with the cystoscope before operation is done.

The treatment consists in excision of the diverticulum, provided the patient can stand operation The task is often a difficult one, as the inflamed sac becomes firmly adherent to the structures among which it lies and may be in a most inaccessible situation. If operation is not done, regular catheterization should be instituted, with frequent bladder lavage.

B. M. B. Male, aged twenty-two. Stitcher in shoe factory. M. G. H., No. 216903. Entered hospital for the second time August 21, 1917. Three years ago was in the Eye and Ear Infirmary with suppurative otitis media. Otherwise has always been well until present trouble began. One year ago, one week after intercourse, he noticed a purulent urethral discharge. This lasted for a week. During this time he had great frequency and urgency, and urination was followed by a few drops of blood.

The discharge ceased, but the frequency, urgency, and hematuria have persisted, with periods of improvement, for the past year. Frequency now five or six times during day, same at night.

He was cystoscoped in the Out-patient Department, but his bladder was so irritable that he was sent into the House for cystoscopy under spinal anesthesia. The bladder was found to be contracted and very 
much inflamed. Both ureters appeared normal. Catheter passed to left kidney drew normal urine. On right, catheter was obstructed 1? inches up ureter, opposite a deep retraction in the bladder wall. 'He was thought to have right renal tuberculosis and guinea-pigs were inoculated. They were all negative. In August he re-entered the hospital. He was

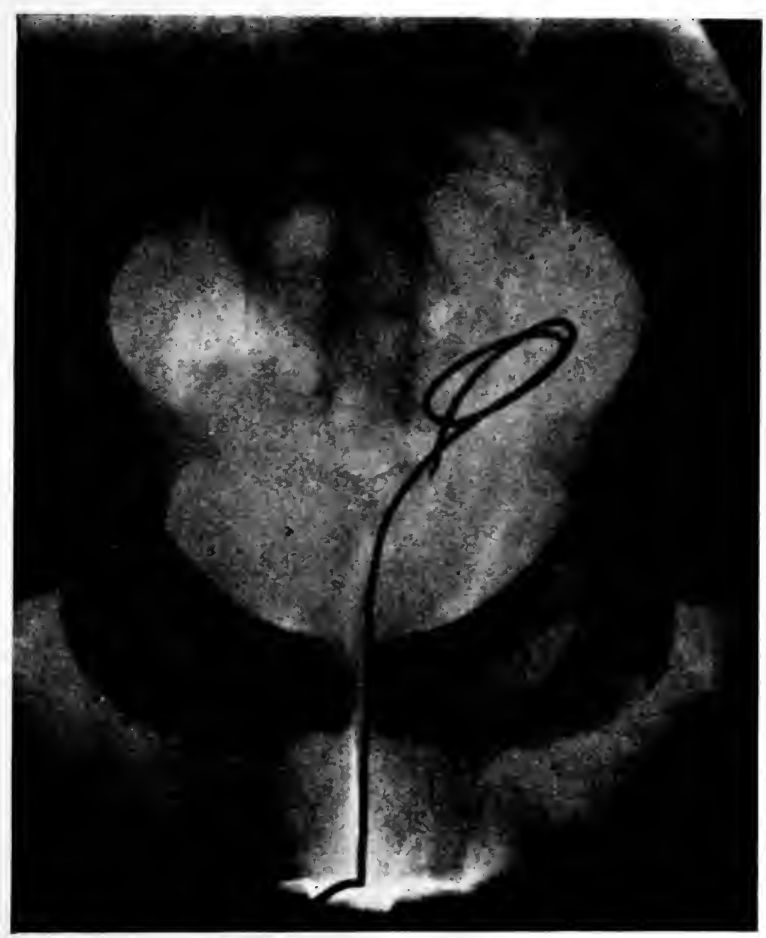

Fig. 2.-Radiographic catheter coiled up in diverticulum of bladder (Mass. General Hospital).

found to have a residual urine of 3 ounces. Cystoscopy showed a diverticulum just outside the right ureter. Catheters passed to both ki.lney's and drew normal urine. A radiographic catheter was coiled up in the diverticulum and $x$-rays taken, which proved the diagnosis (Fig. 2 , Phthalein test: Total function 20 per cent. first hour, 15 per cent. second 
hour. Gonococcus complement-fixation test strongly positive. Wassermann negative.

Operation: Median suprapubic incision, bladder distended. Peritoneum stripped off bladder. Right side of bladder freed from pelvic wall, disclosing a thick-walled diverticulum which was adherent to the right ureter. The diverticulum was freed as much as possible. The bladder was entered accidentally where it was thinned out close to the orifice of the diverticulum. A bougie was passed up the right ureter, the mucosa of the diverticulum was cut away from its firmly fixed base, and the greater part of the sac removed. The bladder was closed except near the top, where a tube was inserted. Rubber tissue wicks were placed to site of diverticulum.

The patient made an excellent convalescence and was discharged in three weeks. He had no residuum and urinated normally.

Fistula and Cyst of the Urachus.-The urachus, which in the middle third of intra-uterine life carries the urine from the bladder to the allantois, later closes and persists as a fibrous cord extending between bladder and umbilicus just anterior to the parietal peritoneum. Occasionally the canal remains patent, and allows urine to escape at the umbilicus. If the bladder end remains open while the umbilical end closes, the condition becomes similar to diverticulum of the bladder. Rarely it becomes obliterated at both ends, but not in the middle, and a cyst is formed by the secretion of its lining epithelium.

The treatment of any one of these anomalies consists of excision of the remains of the urachus.

Exstrophy of the bladder is the result of incomplete closure of the anterior abdominal wall and of the pelvic girdle as well. The bladder everts on to the surface of the abdomen; the trigone appears as a rosette of mucous membrane into which the ureters open. The sufferer from this malformation is, of 
course, bathed in urine; the exposed mucosa ulcerates and infection of the ureters and kidneys results. The condition demands operative interference; good results are not easy to obtain, but are not beyond expectation.

There are several ways of meeting the situation. The Maydl operation, which has been done a number of times, implants the trigone and ureters in the anterior wall of the rectum. Various operations have been devised to form a pouch out of a piece of intestine, and lead the ureters into this sac. Charles H. Mayo points out that the formation of a pouch gives an infected cavity, and says that, rather than trying to get a sterile substitute for the bladder, one should endeavor to prevent renal infection by the method of transplantation of the ureters. He describes an operation which has been done in the Mayo Clinic thirteen times, with one operative death. It consists of embedding the ureters in the wall of the sigmoid for $1 \frac{1}{4}$ inches before bringing the end into the lumen of the gut. By implanting the ureter into the sigmoid the urinary stream is diverted and the remains of the bladder may be removed entirely. Removal of the rudimentary penis may be necessary to allow good closure of the abdominal wall. The operation should be undertaken between the ages of seven and fourteen.

Epispadias.-Incomplete closure of the urethral wall on the upper surface of the penis. This is a rather unusual condition, requring some sort of plastic operation, depending upon the type of epispadias. A transposition of parts has taken place; the corpora cavernosa underlie the corpus spongiosum 


\section{6 AN OUTLINE OFF GENITO-URINARY SURGERY}

and urethra. The inability to impregnate and the inability to urinate in a neat and proper way are the reasons for attempts at cure. Just before puberty is the best time for operation. The incontinence sometimes coexistent with the physical defect may be relieved by training the bladder. By starting and stopping micturition at the word of command, the patient comes to acquire control of his vesical sphincter (Barney).

Hypospadias may be "balanitic" - that is, just behind the glans-or the opening may be at the penoscrotal angle or in the perineum. In extreme cases the scrotum is split and the condition suggests hermaphroditism. The balanitic type is best let alone. In the more severe types the fibrous bands along the under surface of the penis cause the organ to curve downward and prevent erection. They should be operated upon before puberty. Several operations may be necessary. The penis must first be freed from the fibrous bands along its under surface. Then, by one of the several methods which have been suggested, the urethra is carried out to the head of the penis.

Various substitutes for the urethra have been used to supply the missing portion. The appendix and the saphenous vein have both been transplanted to the penis, but have not always survived. Probably the best operation consists in freeing a flap of skin on the under surface of the penis, folding it back upon itself so as to make an epithelial lined cavity, and covering the raw surface by another flap.

Complete success is difficult to obtain; the patient must 
be prepared for several operations. Unless the condition is relieved, coitus may be impossible and impregnation is almost certain to be so. In order to complete the series of operations before puberty it is well to do the first one when the child is six or eight years old.

Undescended Testis.-In many infants the testes do not descend completely into the scrotum until some time after birth. If descent has not occurred by the end of the second year, the chances of its occurring spontaneously are very small. It is probably wise not to interfere until the serenth or eighth year unless the accompanying hernia makes earlier operation necessary. Attempts to push the gland down by means of trusses should not be made. If by the seventh or eighth year the testis is not in the scrotum, operation should be resorted to.

The reasons for operation are: (I) cosmetic; (2) the undescended testicle is tender and is constantly liable to injury; (3) probably because of the constant pressure upon the undescended testis the spermatogenic cells atrophy and sterility upon that side results. The interstitial cells continue to flourish, however, and to supply the internal secretion which is necessary for full development and for sexual desire. Bilateral cryptorchids are usually sterile, although not impotent. The evidence that undescended testes are especially liable to malignant degeneration is disputed. Retained, or abdominal testicles, should be operated upon as well as those in the inguinal canal (Keyes and Mackenzie).

The operation as described by Bevan laid the foundation 
upon which various modifications have arisen. When shortness of the cord does not allow the testis to be placed in the scrotum without tension, the structure at fault is not the vas,

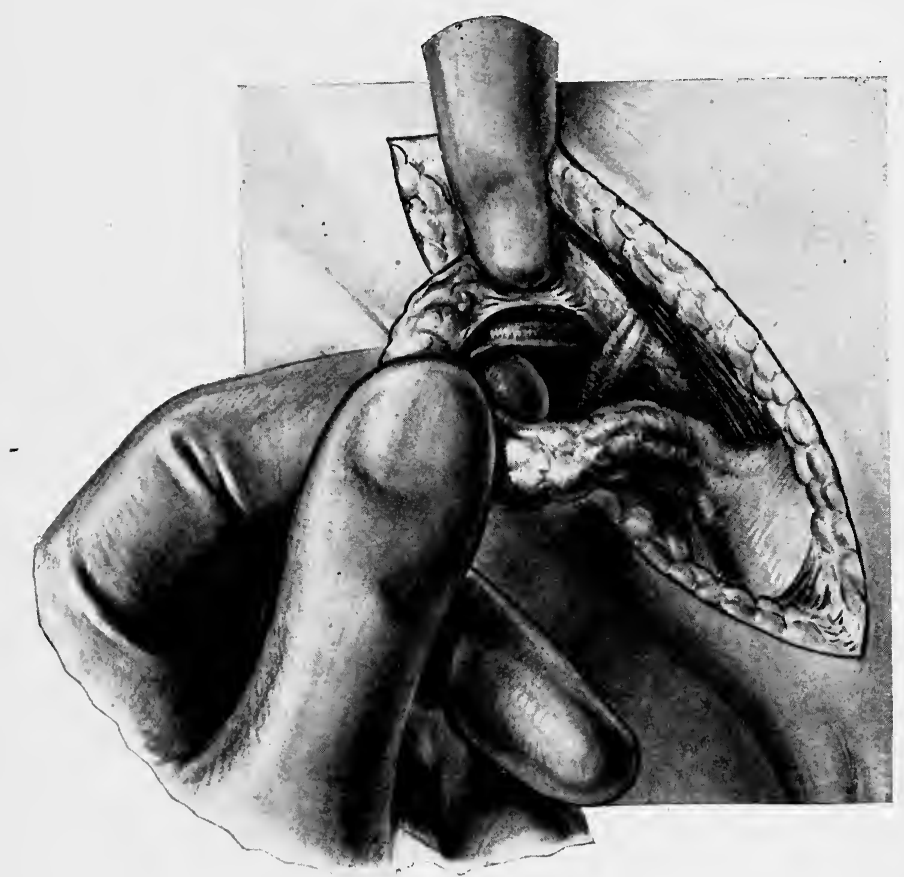

Fig. 3.-Operative treatment of undescended testicle. Stripping the cord off the peritoneum so that it may be drawn down more easily. The testicle may be carried down behind the deep epigastric vessels, in order to do away with the angle in the cord caused by its position in front of the epigastrics.

but the spermatic vessels. Bevan recognized this fact and lengthened the veins by snipping the adhesions which bound the loops together. He also freed the vessels from the peri- 
toneum behind which they lay, and thereby gained additional length. If these means did not suffice, he cut the spermatic veins and even the artery, depending upon the artery of the

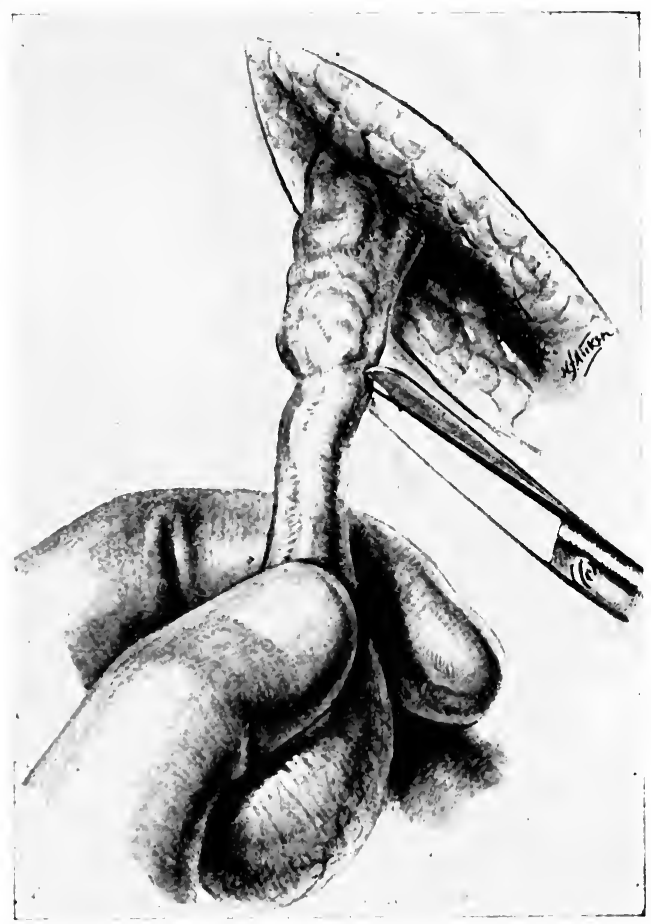

Fig. 4.-Operative treatment of undescended testicle. Lengthening the cord by snipping the ardhesions which bind together the loops of the veins.

vas to nourish the testis. Although sloughing rarely follows this interference with the blood-supply of the testis, the late results of division of the spermatic vessels are shown by Mixter to be far from good. Atrophy nearly always results. 
Other writers have suggested that division of the deep epigastric vessels, over which the spermatic cord is looped, or carrying the testis with its cord behind them, will give a straighter course to the spermatic cord and will thereby allow the testis to be placed in the scrotum.

Mayo, C. H.: The Surgery of the Single and Horseshoe Kidney, Ann. Surg., 1913, lvii, pp. 511-521. Exstrophy of the Rladder and its Treatment, Jour. Amer. Med. Assoc., 1917, lxix, No. 25, 2079.

Lower, W. E.: Diverticula of the Urinary Bladder, Jour. Amer. Med.

Assoc., December 5, 1914, lxiii, 2015-2020.

Савот, Н.: Some Observations upon Diverticulum of the Bladder, Boston Med. and Surg. Jour., 1915, clxxi, 300-302.

Thомas, G. J.: Diverticula of the Urinary Bladder, Surg., Gynec., and Obst., 1916, xxiii, p. 378.

Gardner, J. A.: Diverticulum of the Bladder, Jour. Urology, 1917, i, 439. Barney, J. D.: An Operation for the Relief of Epispadias in the Male, Surg., Gynec., and Obst., November, 1916, 594-597.

Bevan, A. D.: A New Operation for Hypospadias, Jour. Amer. Med. Assoc., 1917, lxviii, 1032.

Tномpson, J. E.: Modern Operations in Hypospadias, Surg., Gynec., and Obst., 1917, xxv, 411.

Mixter, C. G.: Undescended Testicle in Children, Boston Med, and Surg. Jour., 1916, clxxv, 631.

Eisendrath, D.: Undescended Testicle, Ann. Surg., 1916, lxiv, 324-328. Keyes, E. L., JR., and Mackenzie, D. W.: The Operative Treatment of Cryptorchidism, Jour. Amer. Med. Assoc., 1917, lxviii, No. 5, 349351. 


\section{CHAPTER V}

\section{DISEASES OF THE PENIS}

Chancroid.-An ulceration caused by the Ducrey bacillus and acquired by intercourse. Chancroids, are frequently multiple. The typical chancroid is an ulceration irregular in shape, covered with slough, and without induration. It spreads by undermining the skin at its periphery; the undermined skin melts away and leaves a shallow scallop in the outline of the ulcer. Inguinal adenitis is a usual accompaniment; the bacillus of Ducrey is the most frequent cause of "bubo."

The incubation period of chancroid is short-about three days. The treatment consists in cleanliness and in the application of powerful germicides. Crude carbolic acid, followed by nitric acid, is generally sufficient to stop the infection. The acids are applied with a cotton-tipped applicator; the spaces beneath the skin edges must be thoroughly wiped out. If in spite of this treatment the ulcerative process continues, the patient should be anesthetized and the ulcer cauterized with the actual cautery. This will always cure, although two or three applications may be necessary. A dusting-powder, such as bismuth subgallate, may be used by the patient. 
Bubo.-Treatment.-Prompt sterilization of the ulcer will often prevent further developments in the inguinal glands. With the cutting off of this constant supply of fresh infection the glands may be able to overcome the infection already present. If they cannot do this, they will proceed to break down in a matted, necrotic mass. Poulticing hastens the process of resolution, and as soon as fluctuation becomes evident the abscess should be incised, the pockets thoroughly broken up, and the cavity drained. Keyes advises the use of argyrol crystals dusted into the cavity. It would seem probable that packing the cavity with some of the newly developed antiseptics, allied to Dakin's solution, would cause a rapid disappearance of the slough and hasten granulation.

Chancre.-The initial lesion of syphilis. Incubation period about two weeks, although it may be much longer (six to eight weeks). Chancres are usually single, but may be multiple. They are characterized by a rather clean surface, which tends to be elevated rather than depressed; the chancre if small has beneath it a button-like induration; if large, the tissues about it present a narrow zone of smooth, hard induration. The lesion remains about the same for a number of days if untreated, and is not painful. The inguinal glands have a shotty feel; they remain discrete and do not mat together as they do when infected by Ducrey's bacillus.

The diagnosis may be made definitely by the finding of the Spirochætæ pallida in scrapings from the surface of the 
ulcer. This is best done with a microscope equipped with a dark stage; some experience in their recognition is necessary. There should be in every medical center someone familiar with this work to whom suspicious cases may be sent for diagnosis. It is wrong to wait for secondary symptoms or for a positive Wassermann reaction, as the chances of cure are so very much greater if the disease is treated while still in the primary stage. Spirochetes have been demonstrated in the central nervous system during the early secondary stage; it is highly desirable to start treatment before this point is reached. Treatment for syphilis should not be begun until the diagnosis is fairly positive; at the same time every effort should be made to have this diagnosis established before the secondary symptoms appear.

The cases which are most misleading are those due to mixed infection, which begin as undoubted chancroids, and suggest syphilis only when the secordary symptoms appear. Beware of chancroids which develop induration along their edges! Unless chancroid responds to treatment within a few days it is wise to have a dark stage examination made. One injection of salvarsan in the primary stage is worth ten injections later. The treatment of syphilis will not be considered in this book.

Venereal Warts (Condylomata).-These are fungus-like growths occurring usually in the coronal sulcus and upon the inner surface of the prepuce in the male; in women, very profuse growths occur, covering the labia and extending to the perianal region. They are, so far as is known, not of 
infectious origin, but are the camp followers of venereal disease. In other words, the moist condition of the subpreputial space induced by a chronic discharge seems to be the usual predisposing cause. Cleanliness and dryness discourage their growth. They may be snipped off, and the bases cauterized with silver nitrate, or may be touched with glacial acetic acid. The high-frequency current cleans them up very quickly. Calomel powder has seemed very satisfactory as a drying agent.

Condylomata of a broad, flat type are sometimes syphilitic in origin. They occur most frequently about the anus. In all such cases a Wassermann test should be made.

Balanitis.-A non-specific infection of the subpreputial region, due to retained urine which has decomposed, or secondary to a urethral infection. For the former, retraction of the foreskin, bathing twice a day with soap and water, and the application of boric acid ointment are usually sufficient. Frequent attacks are an indication for circumcision.

For balanitis incident to urethritis, subpreputial irrigation with a small catheter and hot soaks are of value. As the edema subsides, drainage improves and the inflammation quiets down.

Phimosis.-A condition of tightness of the prepuce, making retraction difficult. A prepuce may be long-redundant -but phimosis does not exist unless retraction is interfered with. The foreskin should be easily retractable during erection. In some babies the foreskin becomes adherent to 
the glans without being tight. These adhesions should be broken and the prepuce retracted and cleansed every day.

Circumcision-Indications.-It is not our belief that every male baby should be circumcised. The operation should be done, even in boys a few months old, if the prepuce cannot be easily retracted. Adhesions between prepuce and glans may form unless the foreskin can be retracted and the glans penis kept clean.

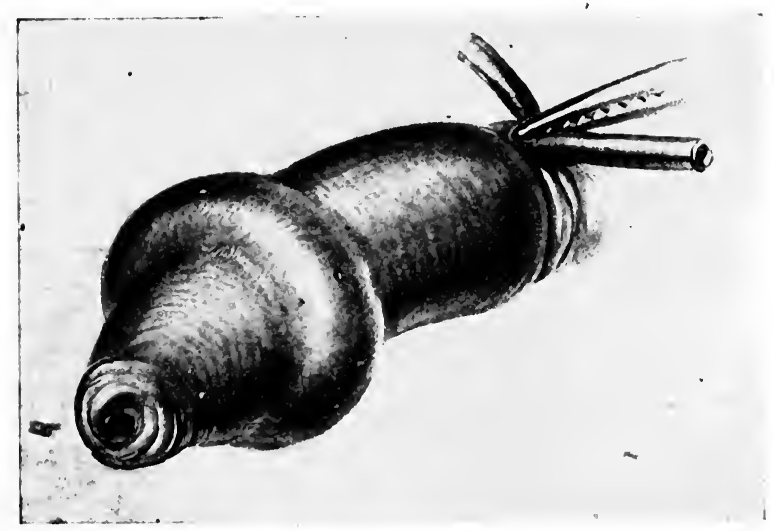

Fig. 5.-Tourniquet in place. Ring of novocain infiltration around penis.

The value of circumcision as a cure for enuresis and for various manifestations of nervousness is doubtful, unless there is a condition of irretractability which requires circumcision for itself. Phimosis is a definite indication for operation, and a redundant prepuce which tends to retain urine or which is easily chafed is much better out of the way.

Technic of Circumcision.-In babies ether should be given. We have found the following technic to be satisfac- 
tory: The prepuce is split dorsally, the dog's ears trimmed off, all bleeding points tied, and the skin and mucous membranes

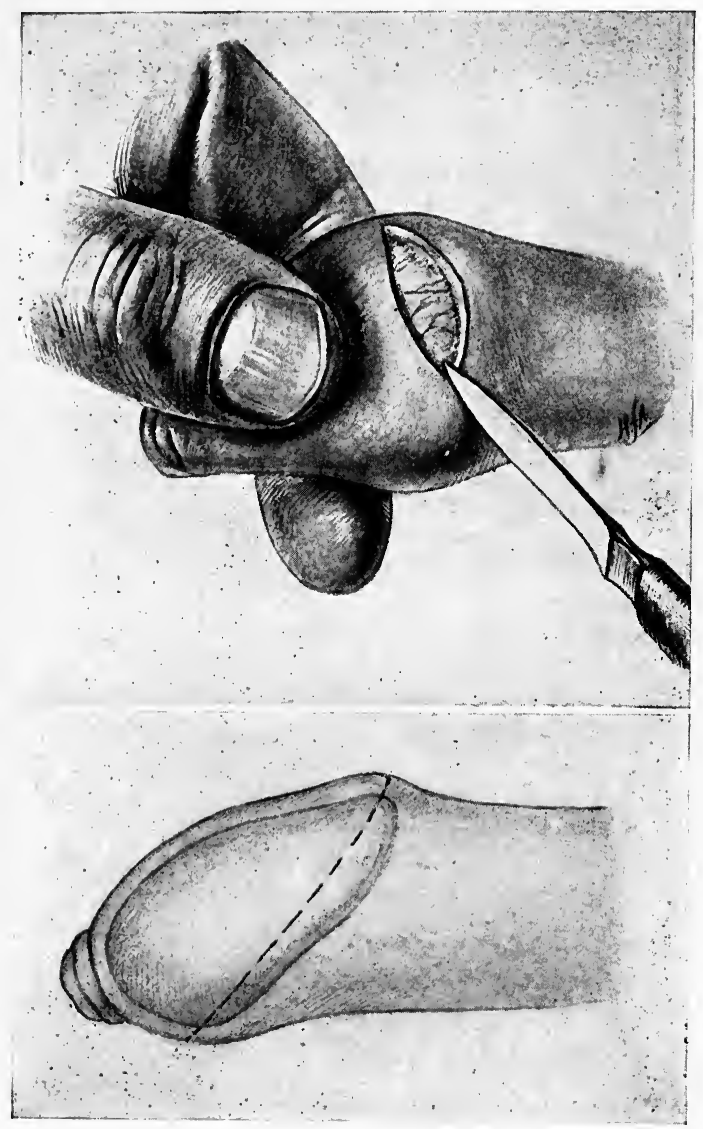

Fig. 6.-Upper: Skin incision begun. Lower: Line of skin incision. united by two continuous sutures of No. I plain catgut, backsewing for half the circumference. A dressing of boric acid ointment is kept on the penis. 
In adults, local anesthesia has given excellent results. A tourniquet is put around the penis as near the pubes as possible. Novocain ( $\mathrm{r}$ per cent.) and adrenalin are used. This solution is injected under the skin of the penis at the level and following the lines of the proposed skin incision. If the prepuce can be retracted, a second line of injection is made

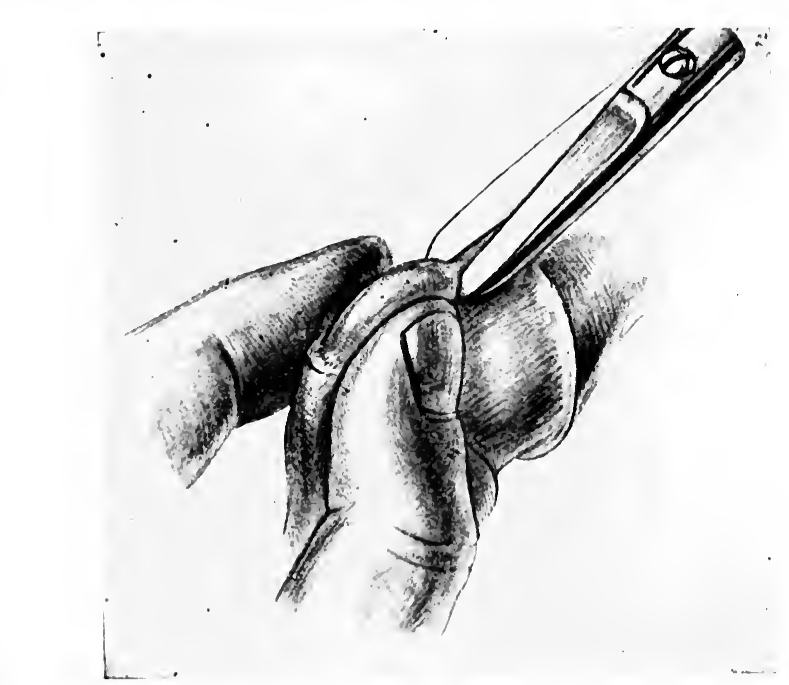

Fig. 7.-Skin incision completed. Incision through mucous membrane begun.

beneath the mucous membrane about $\frac{1}{2}$ inch from the coronal sulcus, going entirely around the penis. 'The skin incision is then made at a level which allows the new prepuce to just cover the corona. The incision is carried around the penis, and if the prepuce is not retractable and the second line of injection has not been made, novocain is in- 


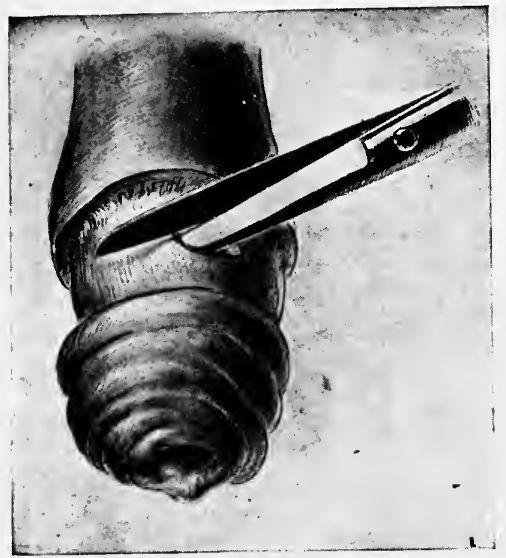

Fig. 8.-Circumcision of inner layer of prepuce.

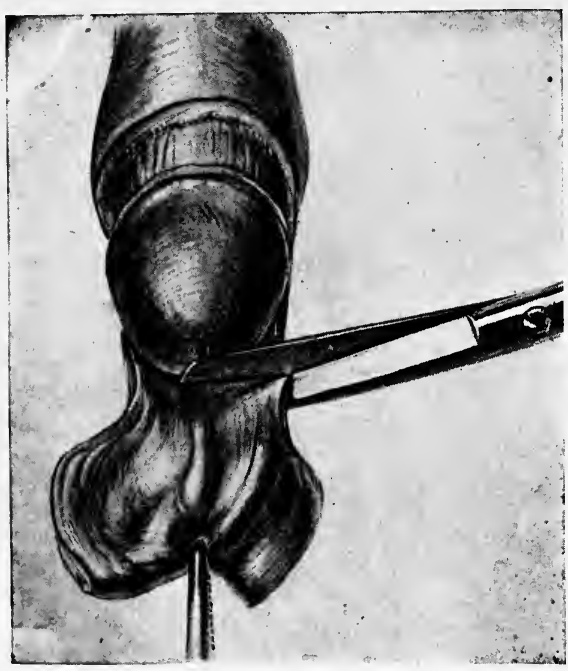

Fig. 9.-Completion of circumcision.

jected into the mucous membrane of the prepuce from its subcutaneous aspect. The mucous membrane is then cir- 
cumcised at about $\frac{1}{2}$ inch from the corona and the ring of prepuce removed. All visible veins, whether bleeding or

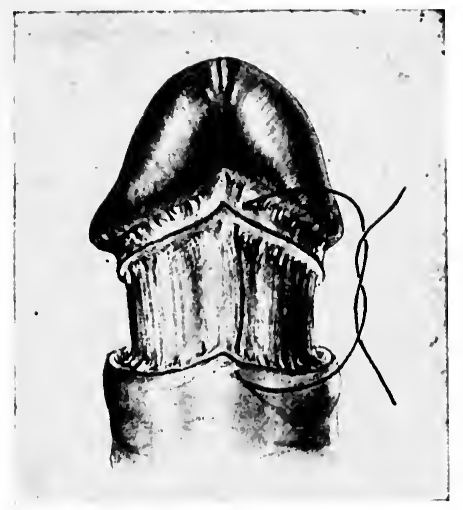

Fig. 10.-Suture of frenum to median raphé.

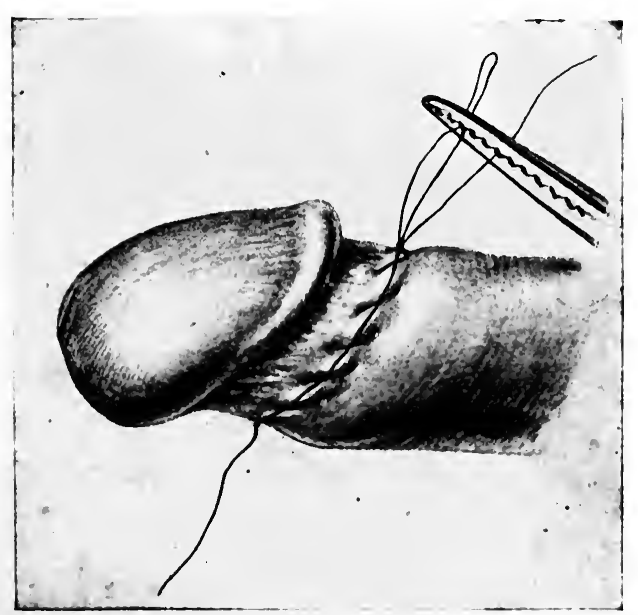

Fir. 11.- Half of suture completed.

not, are ligated with plain catgut. The median raphe on the lower surface of the penis is sutured to the frenum with 


\section{AN OUTLINE OF GENITO-URINARY SURGERY}

a suture of No. I chromic catgut. The skin dorsally is sutured to the mucous membrane in the middle line, and with these two points fixed, the union of the skin to mucosa is completed either by running or by interrupted sutures. Interrupted sutures may have their ends left long in order to tie them over a roll of gauze covered with boric acid oint-

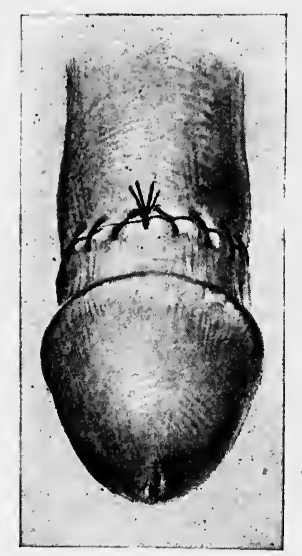

Fig. 12.-Suture completed. After healing takes place the line of suture should lie close to corona.

ment which covers the line of incision. A dressing of gauze supported by figure-of- 8 strapping of adhesive plaster gives support so that the patient may go about his work without too great inconvenience.

Paraphimosis.-A tight prepuce is retracted and becomes caught behind the corona. Efforts of the patient to draw it over the glans fail; the glans becomes edematous and the encircling band so much the tighter. If allowed to go on in 
this way, the tightest part of the prepuce sloughs and the constriction is thereby released.

To reduce a paraphimosis, the operator faces the patient and, having lubricated the glans penis with vaselin, clasps the shaft of the penis between first and second fingers of both hands, and with his thumbs gently forces the edema out

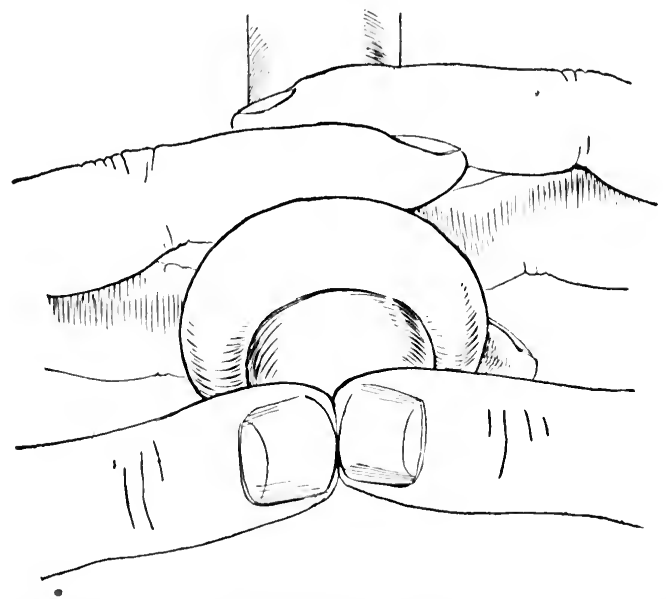

Fig. 13.-Method of reducing a paraphimosis. The thumbs express the edema from the glans penis and push the glans through the ring of prepuce, while the latter is pulled forward by the interlaced fingers.

of the head of the penis. Pushing with the thumbs and pulling with the fingers he can almost always succeed in reducing the paraphimosis. If reduction fails, the constricting band may be cut, thereby releasing the prepuce.

Cancer of the penis occurs occasionally in men as young as thirty, but usually in men fifty or more years of age. It is alleged that cancer of the penis never appears in those who 
have been circumcised. The growth springs, as a rule, from the glans or inner aspect of the prepuce, and appears in the preputial orifice as a fungus-like, knobby, often ulcerated growth. Venereal warts and tertiary syphilis are the only lesions likely to be confounded with it.

The treatment is amputation of the penis, which should be accompanied by dissection of both groins. The urethra should be cut longer than the stump to allow for its retraction. It should be split and sutured over the denuded ends of the corpora cavernosa. Good healing is aided by instituting perineal drainage. A soft-rubber catheter is passed to the bladder. The outer end is grasped with a pair of curved hemostats, and pushed into the urethra as far as the perineum. The hemostat is then turned so that the tip may be felt through the skin and the overlying tissues incised. The end of the catheter is drawn out through this opening and fastened in the proper position. The incision closes promptly when the catheter is removed. (Barney.)

Robins, F. W., and Seabury, F. P.: Treatment of Chancroid, Jour. Amer. Med. Assoc., 1917, lxix, 1217.

Pusey, W. A.: Erosive or Gangrenous Balanitis, Jour. Amer. Med. Assoc., 1917, lxix, 1080.

Cunningham, J. H.: Operative Treatment of Carcinoma of the Penis, Surg., Gynec., and Obst., 1914, xix, 693-699. 


\section{CHAPTER VI}

\section{DISEASES OF THE URETHRA}

Gonorrhea.-Infection of the male urethra by the gonococcus of Neisser is acquired only by intercourse. The incubation period may be as little as twenty-four hours, but is much more often two or three days, and it may even be longer before the evidences of disease are noticel. The history of exposure followed by the appearance of a discharge is strongly suggestive of gonorrhea, although the diagnosis should be made definitely by the finding of the organisms in smears (see Chapter II).

Measures directed toward the prevention of gonorrhea have included the use of the condom or the application of some germicidal solution to the parts exposed. In the Navy methods of applying venereal prophylaxis have consisted of anterior urethral irrigations of potassium permanganate solution which were given to men who reported exposure upon their return from shore leave, or of encouraging the men to use the so-called sanitary tube, which contains an ointment made of

Calomel.................. 50 grams

Vaselin liq................ so c.c.

Lanolin.................. 70 grams 
This tube is collapsible and has a urethral nozzle. Immediately after exposure part of the ointment is injected into the urethra and the rest is rubbed on the outside of the penis. ${ }^{1}$ Both these methods have greatly decreased the incidence of venereal disease; the proportion of cases in which prophylaxis fails to prevent disease increases with the interval which elaspses between exposure and treatment. After six hours have elapsed the percentage of failures increases rapidly.

The regulations of the Surgeon General of the Army require a preliminary washing of the genitals with soap and warm water, the injection into the urethra of 2 -per cent. protargol or 20 per cent. argyrol, which must be retained for three minutes, and the rubbing of calomel ointment ( 30 per cent. in benzoated lard) into the surface of the entire penis. The parts are then to be wrapped in soft paper to protect the clothing.

Postmortem examination of criminals who have been inoculated before death has shown that thirty-eight hours after inoculation the gonococci had only just begun to effect an entrance between the epithelial cells. At the end of three days the inflammatory process was well under way. ${ }^{2}$

Aborting.-It seems unreasonable to expect success from any measure undertaken with a view to "aborting" an attack of urethritis, since the attack would naturally not be discovered until the organism had penetrated deeply enough

${ }^{1}$ Henry: The Military Surgeon, May, 1912, xxx, 520.

2 Finger, Gohn, and Schlagenhaufer: Arch. f. Derm. u. Syph., 1894, xxviii, 277, abstracted by Keyes, Dis. of the Gen.-urin. Organs, 1911, p. 150 . 
to set up a diapedesis of leukocytes. Ballenger and Elder, however, report good results in very early infections (within the first twenty-four hours of the discovery of the discharge) obtained by sealing up the meatus with collodion after the anterior urethra had been injected with 5 per cent. argyrol solution. They describe the following technic:

"The patient should be placed in a reclining posture. The penis should be washed and dried, then surrounded with a sterile towel. A syringe holding 25 minims is used to inject this amount of medicament into the ürethra. A larger amount does not seem to give any better results and is more likely not to be held in the urethra for the necessary time. Next, the canal is compressed with the left hand while the meatus is dried. Collodion is then applied with a camel's hair brush over the meatus and the glans penis for $\frac{1}{4}$ inch around the meatus. The compression of the urethra should continue for about five minutes until the collodion is thoroughly dry and feels hard to the touch. It is important to use plain collodion, U. S. P., I910, which contains no castor oil or balsams and which feels hard when it dries. No cotton should be placed over the meatus, as it will not hold well and is unnecessary. When the collodion is thoroughly dried the urethra should be released and a condom containing a small amount of absorbent cotton should be placed on the penis to protect the patient's clothing in case the collodion should break and allow the escape of the medicament sealed in the urethra.

"The patient, who should empty his bladder just before 
the treatment is applied, should be told not to drink anything for a few hours and to allow the drug to stay in the urethra four or five hours, if he is not compelled to urinate before this time has elapsed. When he wishes to void, the anterior part of the urethra should be compressed and pressure applied over the collodion, causing it to break at some point that affords a line of cleavage, by which the collodion may then be pulled off.

"After allowing the escape of the medicament, the patient should drink freely of water for the remainder of the day, so as to flush the urethra with bland, unirritating urine. The following morning very little water or other liquids should be taken, in order to prevent a distended bladder from allowing the medicament to stay in the urethra for the full time.

"The treatments may be repeated once a day for five days if conditions are favorable. If the discharge continues or increases, or if organisms can be found in the secretion on the third or fourth day, it may be taken to mean that the germs have extended to a point not reached by the medicament and that this method should be replaced by the usual treatment."

Although I have had no experience with this method, I should feel that it might be valuable in cases which begin mildly. If a case, when first seen, has a well-developed purulent discharge, sealing the meatus would dam back the secretion and cause the infection to extend into the posterior urethra. 
It has been alleged that heat applied by means of a psychrophore is fatal to gonococci in the urethra. The organism is said to be killed by a temperature of $I I 9^{\circ} \mathrm{F}$, a temperature which might conceivably be induced in the urethral wall without causing serious injury. This method has not been adopted by the medical profession generally, and even if it is able to accomplish the death of gonococci in the tissues, the number of cases in which it would be applicable is small. The majority of cases of acute urethritis affect the posterior urethra. In these cases the use of heat in the anterior urethra alone would be futile, and in the posterior urethra would be dangerous.

Treatment.-Gonorrhea is at first a disease of the wall of the urethra. The organisms penetrate the superficial layers of the mucous membrane, causing a general edema and attracting great numbers of leukocytes. If the infection were limited to the flat surface of the mucosa its duration would be brief, for without special centers of resistance the gonococcus would soon be vanquished by the processes of desquamation and leukocytosis. Unfortunately, the organisms enter some of the numerous accessory urethral glands - the peri-urethral glands in the anterior urethra, and in the posterior urethra the prostatic ducts and the seminal passages. Cowper's glands and the utricle of the verumontanum may be infected. Only in from to to 20 per cent. of cases is the infection confined to the anterior urethra. Of the 80 or $9 \circ$ per cent. of cases in which it reaches the posterior urethra a large majority show involvement of the 
prostate or seminal vesicles. The treatment of these complications will be taken up in the chapters devoted to the lesions of these organs. In this chapter only the urethral manifestations of gonorrhea will be discussed.

In the acute stage of urethritis the object of treatment is to allay the inflammation. This is done by forcing fluids, avoiding all irritating drinks, including "tonics," soda water and ginger ale, and spicy or peppery foods. General hygiene is very important; the patient should have plenty of sleep, as little exercise as possible, free bowel movements, and quantities of water. He must avoid exposure to cold. By mouth, potassium citrate, Io grains, ${ }^{1}$ may be given four times a day. Capsules containing ro minims of sandalwood oil, three times a day, taken during each meal to avoid gastric disturbance, are valuable in allaying the irritability of the mucous membrane of the urethra: In very acute cases, with much tenesmus and with blood in the discharge, no local treatment at all should be given until the inflammation has quieted down. Then one may irrigate the anterior urethra through a two-way meatal nozzle with at least 16 ounces of very warm, weak potassium permanganate solution ( $1: 8000$ ) every day or every other day. The value of this treatment lies largely in the effect of the heat upon the blood-supply and upon the metabolism of the mucous membrane. Heat increases the desquamation of the epithelial cells in which

${ }^{1}$ R. Potassium citrate............... 3v;

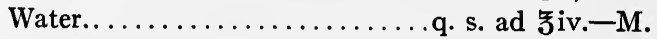

Sig.-Teaspoonful in glass of water four times a day. 
the organisms are embedded, and accelerates the migration of leukocytes. Potassium permanganate, through its power to dissolve the mucus which coats the urethral wall, improves the drainage of urethral crypts and washes out masses of infectious secretion. Until the very acute stage of urethritis has quieted down no instrument should be passed into the urethra.

After the first few days in very acute attacks, or earlier in the less acute cases, the patient may be instructed in the use of the urethral syringe. He should hold the penis with the second and third fingers of the left hand, leaving the thumb and index-finger free to compress the meatus (Fig. 14). With the syringe he injects not more than I dram of some one of the organic silver salts into the anterior urethra and holds it there for from two to five minutes, depending on the reaction which follows. Argyrol, used at first in a 5 per cent. and later in a ro per cent. solution, and hegonon, in $\frac{1}{8}$ or $\frac{1}{4}$ per cent. strength, have, in my experience, given the best results. The value of these preparations lies in their bactericidal power combined with the absence of irritating properties. The injection should be made three or four times a day.

Inside of two or three weeks the discharge should be uncier control, the vesical tenesmus, if such exists, should be allayed, and the urine should show less opacity and more shreds. The clearing of the urine is due to the disappearance of the general infection of the mucosa, while the shreds are caused by exudate from residual ulcerations and from 
infected glands. At this time it is well to ascertain whether the process has extended to the posterior urethra (80 to 90

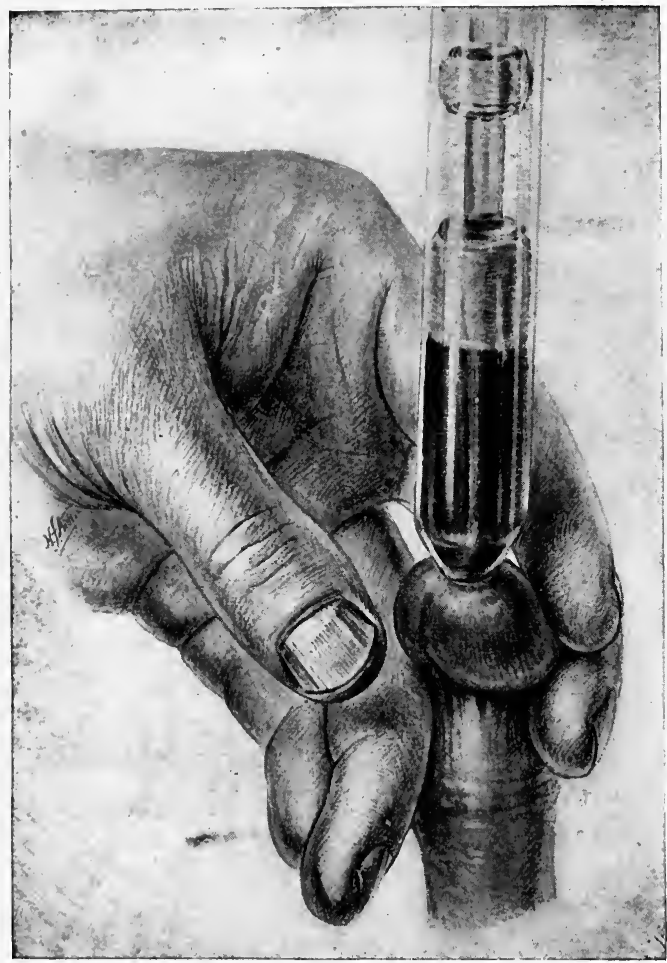

Fig. 14.-Method of holding penis while making urethral injectrons. Thumb and forefinger free to compress lips of meatus as soon as syringe is withdrawn.

per cent. of all cases of urethritis do extend back of the cutoff muscle).

There seems to be a belief among medical men that if the patient urinates into two glasses and the second urine is 
clear, its clarity is evidence that the infection is confined to the anterior urethra. This idea is quite wrong, for in the less severe infections of the posterior urethra the secretion of that portion of the canal is carried out with the first few drams of urine and the second urine is clear. It is only when the posterior involvement is severe enough for the pus to run back into the bladder that the second urine is cloudy.

To determine accurately whether the posterior urethra is involved the anterior urethra should be thoroughly irrigated with potassium permanganate solution of considerable strength ( $1: 3000)$; the patient then urinates into two glasses. If the first urine is perfectly clear and sparkling, there is no posterior urethritis. If the first urine contains shreds colored pink, they come from the anterior urethra; if the shreds are white or if the urine is cloudy (and does not clear upon the addition of acetic acid) the infection has extended to the posterior urethra. This test should be applied frequently. If posterior urethritis exists it must be treated locally as soon as the condition of the urethra will permit the passage of a catheter without irritation. The rule in the treatment of gonorrhea is to push the treatment as fast as possible without traumatizing the urethra or adding to its irritability. If, therefore, a posterior urethritis exists, and the anterior urethra has acquired a tolerance to the infection as evidenced by diminution of the discharge, a small soft-rubber catheter may be passed with exceeding gentleness until it just passes the cut-off, and through this 


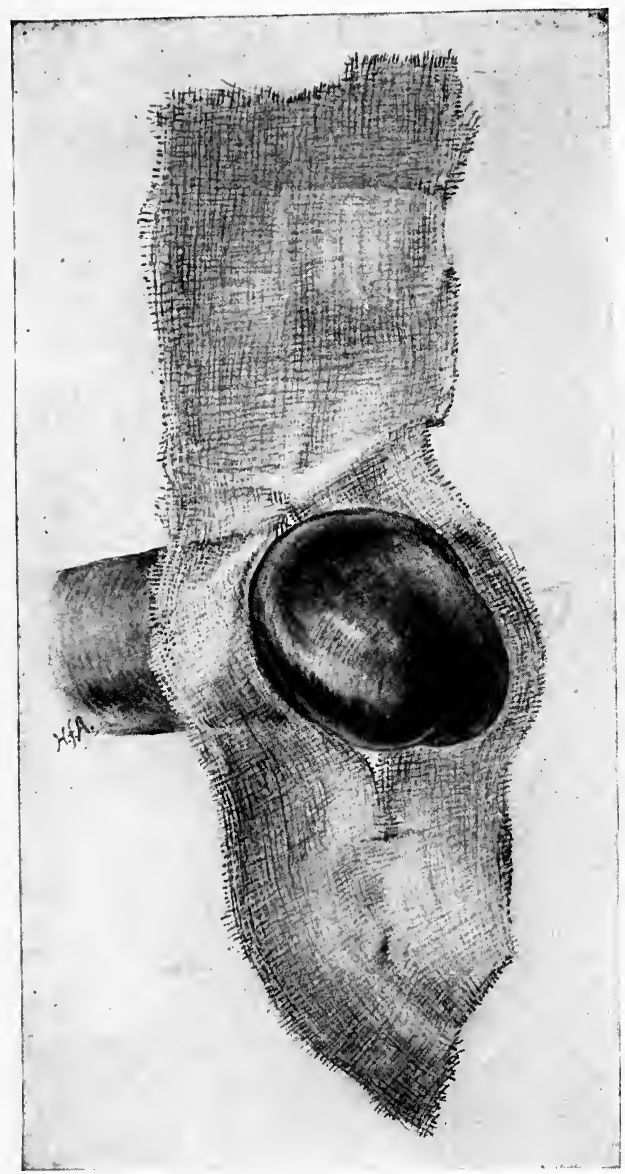

Fig. 15.-Dressing for penis to be worn during acute stage of urethritis. Prepuce retracted.

catheter, again with gentleness, 8 or I 2 ounces of potassium permanganate solution of about $\mathrm{x}: 5000$ may be put into the bladder. The anterior urethra should be irrigated with 
another 12 ounces as the catheter is being withdrawn. This may be carried out every day, and should be done at least twice a week. The patient meanwhile continues the use of argyrol and sandalwood oil. Some men prefer to fill the bladder by gravity. An irrigator is suspended 4 feet above the treatment table. The tip of the irrigator tube is held tightly against the urethral meatus, and while the patient relaxes the cut-off muscle (as if urinating), hydraulic pressure forces the fluid into the bladder.

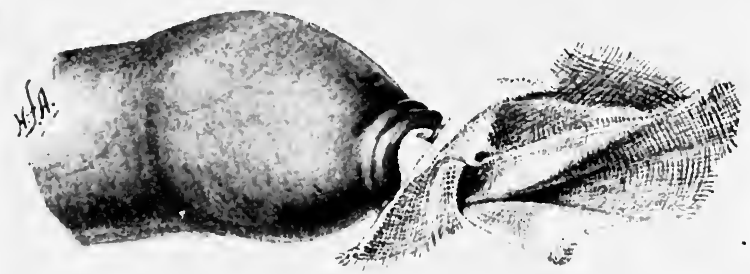

Fig. 16.-Dressing for penis to be worn during acute stage of urethritis. Prepuce drawn down over gauze.

Under this treatment the urethritis should clear up, often entirely. At the end of four to six weeks the urine should be clear, with perhaps a few shreds.

The number of injections of argyrol per day may be gradually reduced and finally stopped altogether. The mucoid discharge which often persists will steadily diminish as the urethral mucosa regains its normal condition. Hot irrigations with potassium permanganate or silver nitrate solution twice a week will hasten the process. Persistent shreds 
may be cleared up by gentle massage of the anterior urethra upon a sound.

The patient is allowed to assume his regular ways of living, and if, after a month without treatment, there is no discharge, the urine is perfectly clear, and there is no infection of prostate or vesicles, he may be discharged as cured.

The recurrence of discharge after cessation of treatment means infected glands in the mucosa, or more generally an infection of the prostate or seminal vesicles.

One should not wait for this outbreak before examining the prostate and vesicles. In this regard the medical profession is either careless or uninstructed, for many a case of urethritis is quickly cleared up and discharged as cured by a medical attendant who has never examined the patient by rectum. These cases later have recurrences in the form of gleet, or, believing themselves to be cured, marry and infect their wives.

No case of gonorrhea should be discharged until a careful, digital examination has been made of prostate and vesicles, and a smear of the expressed secretion examined microscopically for the presence of pus.

Not by any manner of means do all cases of urethritis run so smooth a course as has been outlined. Of two cases treated in just the same way, one will go on to rapid recovery, while the other will continue to have cloudy urine, persistent discharge, and perhaps even an epididymitis. With the case whose urine does not clear up after several weeks of anterior and posterior irrigations, one should palpate the 
prostate and vesicles by rectum and determine whether they present a uniformly soft, elastic outline, or whether they contain areas of induration. The secretion expressed by very gentle massage should be examined for pus-cells. When the second urine is persistently cloudy, the prostate and vesicles are practically always found to be infected. This infection, in my experience, is in the vesicles in the great majority of cases. Infection of the prostate, characterized by a condition of stony hardness or by the presence of areas of infiltration, does not seem to keep up urethral infection as does involvement of the vesicles. When the prostate alone is infected, as determined by the examining finger, the urine not infrequently remains perfectly clear, and the prostate slowly softens down without treatment and without reinfecting the urethra. Invasion of the vesicles is a different matter. The process may vary from the slightest possible infection to one of such extent that the pus bursts through the wall of the vesicle and forms abscesses within the pelvis. Rude or illadvised treatment is followed by dire results-the spread of the infection within the vesicle or its extension to the epididymis. The question arises when to massage the vesicles and when to leave them alone. Each case must be judged upon its merits. If, after three weeks of irrigations, the urine shows no signs of clearing, very gentle massage may be tried. It should be done not oftener than twice a week, so that the reaction excited by the treatment may subside before the next one is due. If the results are good, continue; if the benefits are not obvious, drop massage for 
awhile and confine the treatment to urethral irrigation and rectal injections of hot water. Irrigation can do no harm, but rough or ill-timed massage can raise all kinds of trouble. With the dying down of the vesicular infection the urethritis clears up. The treatment then becomes chiefly that of vesiculitis. (See Chapter VIII.)

Occasionally one meets a case of acute urethritis in which the infection is kept up by a stricture. The discharge is dammed back, and will not diminish until the stricture is dilated. Dilatation of a stricture in the face of acute infection calls for unusual gentleness. The instruments used should be small enough to pass without trauma, and should not be passed through the cut-off muscle. Copious irrigation should follow the dilatation.

Persistence of the discharge from this cause may occur in men who have never before had urethritis. A stricture of considerable resilience may form within eight weeks of the inception of a urethritis. Apparently such strictures form as a result of infection of a peri-urethral gland. They may be diagnosed by the passage of a bougie à boule through the anterior urethra-a procedure which is comparatively harmless if gently done.

N. H. D., age eighteen, contracted his first urethritis ir. July, 1917. His doctor kept him in bed five weeks, as he had fever and great bladder distress. In spite of forced fluids and argyrol injections the discharge continued. On September 15, 1917, he was sent to me. He had a profuse urethral discharge, containing gonococci. The urine was cloudy and contained masses of slough. Frequency two or three times an hour. A small peri-urethral abscess could be felt at the penoscrotal angle. Prostate and vesicles were small and soft. 
He was given potassium permanganate irrigations and argyrol instillations for two weeks without much improvement. A bougie à boule was then passed and a definite stricture, size 21 French, was discovered at the penoscrotal angle. This was dilated with sounds. After three or four treatments the discharge had practically disappeared, although the stricture was still tight to 27 French sound. I ilatation was done whenever the patient came to see me, which was not very often. Meatotomy was required to allow the passage of instruments larger than 27 French. March 1, 1918, there is no discharge, the urine contains but one or two shreds. Sound 29 is tight. Prostate and vesicles are normal. He is cured of his gonorrhea, but the stricture has not yet entirely disappeared.

Chronic Urethritis.-Only a minority of men with acute urethritis consult a physician and follow his treatment to a cure. Many do nothing for the infection, and if the attack is mild, the discharge may cease of itself. Others consult friends or druggists; they get some "sure-cure" medicine, and when the discharge quiets down, imagine themselves well. Still others seek medical advice, are given an injection to use, and, to the shame of the profession, are discharged as cured without a thorough examination. With one of these three stories the sufferer from chronic urethritis presents himself for treatment. His condition has existed anywhere from three months to years. Examination shows a slight colorless or grayish-white discharge which contains epithelium, pus-cells, many odd bacteria, and probably no gonococci. The urine contains shreds and more or less pus. This means urethral inflammation, and the cause of it is either a lesion in the urethra, such as a stricture, or pros. tatitis or vesiculitis. The former is ruled out by the passage of a large bougie à boule or of a sound, size 26 or 28 French. The existence of prostatitis or vesiculitis is de- 
termined by rectal examination, together with the examination of the secretion expressed by massage. If the urethral infection is due to either of these causes, it will be cleaned up by treatment directed against the underlying condition.

In a small proportion of these cases an unusually severe ulceration of the urethra will cause areas of induration or of granulation which keep up a discharge. This condition will be indicated by the persistent presence in the urine of shreds of the long, heavy type.'

For such a condition the passage of sounds is the remedy. A moderate sized sound-24 French, for example-should be tried first. At subsequent visits larger and larger ones must be used until the urethra will take a sound of from 30 to 32 French caliber without gripping. If the meatus is tight it must be cut to admit easily at least a 30 French sound.

Meatotomy is done with local anesthesia. A few minims of cocain or novocain are injected into the glans between the frenum and the meatus. A small knife is passed for $\frac{1}{2}$ inch into the urethra, and the floor of the canal and the skin at the frenal side of the meatus divided until a No. 32 sound will pass. Incision of the urethral floor for about $\frac{1}{2}$ inch from the meatus is necessary. Then the mucous membrane of the urethra is approximated to the mucous membrane of the glans by two stitches of catgut or Pagenstecher, one on either side of the center. This stitch, suggested by Barney, checks bleeding and tends to prevent adhesion of the cut edges (Fig. I7). 
For persistent urethritis irrigations of silver nitrate solution ( $1: 5000$ and stronger) are efficacious. Desquamation is increased; new cells come to the surface, and the shreds disappear.

The question of cure in a case of urethritis not complicated by infection of the accessory glands is easily settled. The

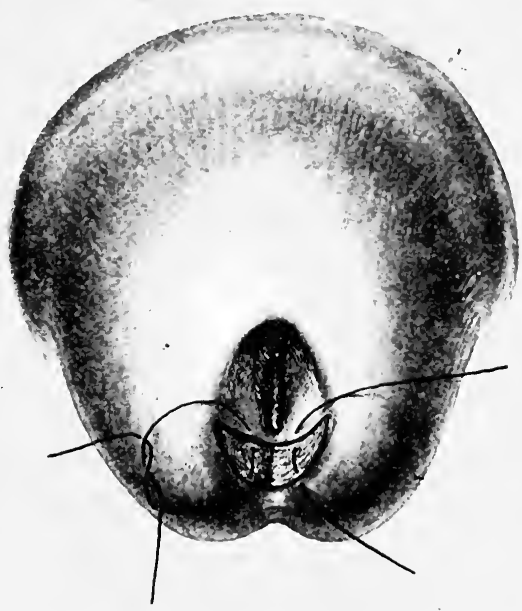

Fig. 17.-Placing of the suture after meatotomy to bring mucous membrane of urethra down to that of the glans penis, thus covering the denuded surface.

necessary criteria are absence of discharge, clear urine, and no evidence of prostatic or vesicular involvement as determined by a microscopic examination of the secretion expressed by massage. The persistence of a rare shred may be disregarded if there is no constriction of the urethra and no 
peri-urethritis to be felt when the urethral wall is palpated upon a sound.

These conditions should be unaffected by a return of the patient to his regular habits. There should be no reaction following exercise or the use of alcohol. The patient should be under observation for a month or six weeks, and if the urine remains perfectly clear he may be discharged as cured.

When the prostate or vesicles have been infected it is much more difficult to say just when a cure has been achieved. This phase of the question of cure will be discussed in the section on Infections of the Prostate and Vesicles (Chapter VIII).

To sum up, the treatment of the acute stage of gonococcus urethritis is largely expectant. Give the urethra every chance to throw off the infection; do nothing to traumatize still further the urethral mucosa. Instillations of argyrol and irrigations with hot potassium permanganate are always safe measures. Continue this policy as long as progress continues. When improvement ceases a focus should be sought and measures taken to eradicate it. "Slow and sure" is better than speedy and sorrowful-and epididymitis.

Non-specific Urethritis.-All urethritis is not due to the gonococcus, even remotely. In certain cases it may be difficult to say whether this organism is responsible or not, especially if in the past there has been an infection with the diplococcus of Neisser.

An inflammatory condition of the urethra, manifested by discharge and by burning on urination, may result from the 
too enthusiastic use of antiseptics. A solution of corrosive sublimate of I : 500 , for example, is well qualified to stir up trouble. Microscopic examination in cases of this type shows almost no pus, but quantities of epithelial cells.

On November 2, 1916, I was consulted by a doctol, thirty-two years of age, who had been exposed to infection one week before. Is a prophylactic measure he had given himself a urethral injection of $1: 5(\%)$ corrosive sublimate. Two days later a mucoid diccharge appeared in which I could find no gonococci. There were many epithelial cells, and the picture was that of desquamating mucous membrane. After washing out the anterior urethra the urine first voided was clear, showing that the posterior urethra was not affected. As the patient had a history of gonorrhea when he was fourteen years of are, this test was valuable in that it helped to establish the fact that the urethritis was not caused hy. the outbreak of an old prostatitis or vesiculitis. This fact wals further proved by the absence of pus-cells from the secretion obtained by prostatic massage.

As the patient was a very nervous inclividual, and would not tolerate sounds, the discharge hung on for three months. By that time it had gradually disappeared. The urine contained a few shreds, and several tiny glands were palpable along the anterior urethra. The complementfixation test was negative on February 12, 1917, so treatment was stopped.

Since then the patient has been perfectly well, with no recurrence of the discharge.

Another kind of non-specific urethrit is occurs in men who have had a gonococcus infection of the prostate in the past. The gonococcus has died out; a low-grade secondary infection persists. Under the spur of some exciting cause, such as excessive drinking, combined perhaps with unusual sexual excitement, these organisms infect the urethra. 'The urethral secretion contains pus-cells, a large number of epithelial cells, many bacteria, but no gonococci. The circumstances 
under which the discharge appears, as well as the patient's guilty conscience, suggest a new infection. Treatment for acute urethritis is instituted. The discharge at once subsides, leaving just enough signs of trouble to tell him that he is not cured. These persist; the morning drop and shreds do not disappear. At this point or earlier the wise physician examines the prostatic secretion after massage and discovers why his remedies have failed. Under treatment with sounds, prostatic massage, and irrigations of fairly strong solutions of silver nitrate $(\mathrm{I}: 3000)$ the urethritis clears up.

J. S. C., aged forty, had a gonococcus infection ten years ago. He has been well since. A week before consulting me he had intercourse. Six days later he noticed a slight discharge. The urine was clean, with a rare shred. No discharge could be seen, though the patient had seen some that morning. Examination showed no stricture of the urethra or involvement of the peri-urethral glands. The vesicles on palpation seemed very full, large, and indurated. The secretion from them showed some pus-cells. After four treatments-urethral irrigation and vesicular massage-all symptoms cleared up and patient refused further treatment.

A similar condition is found in men who have a prostatitis or vesiculitis and who never had a gonococcus infection. Such cases are not rare.

B. C., aged thirty. Has been married seven years, and has 3 children. Five years ago he was said to have had prostatitis. No known cause for it. Three weeks ago was exposed. Two weeks ago he noticed a milky discharge from the urethra. Has had no treatment for it. Urination is much more frequent than normally. Examination shows a slight discharge. The smear shows epithelial cells and leukocytes, but no gonococci. After lavage of the anterior urethra the first urine shows mucous clouds, the second is clear. No stricture to 27 French sound. Silver nitrate wash and passage of sounds do not arouse the infection as they would if the gonococcus were present. The vesicles on palpation seem 
very much distended. Considerable secretion is expressed by massage and shows pus.

After three months of rather irregular treat ment the patient developed a severe epididymitis. Now, four months after the beginning of treatment, there is still some discharge and the urine is full of shreds. There is distinct improvement in his subjective symptoms. Frequency is now almost normal. It first irrigation of the entire urethra was the only measure his urethra would tolerate. Now sounds and vesicular massige are being employed; the discharge is almost gone, the shreds are smaller and fewer in number.

In some of these cases social considerations call for an answer to the question, whether there has been a new infection, or whether the urethritis is the result of an old prostatitis. If the discharge is examined within the first week the absence of gonococci points strongly to the latter. If the patient is not seen until the discharge has largely disappeared, one may be aided in the solution of the problem by the complement-fixation test. A fresh infection which has been going on for a month or six weeks and has involved the prostate or vesicles should give a definitely positive blood test. A negative blood test, given a prostatic infection and a discharge of more than four weeks' duration, is good evidence that the infection is of non-specific origin.

A mild urethral discharge without gonococci, with a considerable proportion of epithelial cells even in the early stage, coupled with a past history of gonorrhea, must always suggest a non-specific urethritis. This holds true even though it follows exposure in a presumably infectious quarter. The source of the discharge in such a case will be found to be either latent vesiculitis or potential stricture of the urethra. In 


\section{4 \\ AN OUTLINE OF GENITO-URINARY SURGERY}

the great majority of cases of non-specific urethritis silver nitrate in solutions of from I : 3000 to $I: 8000$ is the best irrigation.

M. T. H., aged twenty-seven. Unmarried. Clerk. Gonorrhea four years ago, lasting three months. Exposed four weeks ago. For past three weeks the lips of the meatus stick together. No discharge seen. First urine shows one shred; second is clear. Prostate and vesicles normal. Between the meatus and the penoscrotal angle are several ridges distinctly perceptible as a bougie à boule, size 24 French, is passed through them. Complete clearing up of all symptoms and smoothing out of the ridges followed four treatments with sounds and silver nitrate irrigations.

Ballenger, E. G., and Elder, O. F.: The Technic for Sealing Medication in the Urethral Canal, Jour. Amer. Med. Assoc., 1918, lxx, 834. Barney, J. D.: The Management of Gonorrhea, Boston Med. and Surg. Jour., 1916, clxxiv, 740-745.

HaWard, V.: Military Hygiene, Wm. Wood \& Co., 3rd ed., pp. $122-131$.

Lumb, N. P. L.: Five Hundred Consecutive Cases of Acute Gonorrhea, Brit. Med. Jour., October, 1917, ii, 450-452.

Pollack, C. E., and Harrison, L. W.: Gonococcal Infections, Oxford University Press, 1912, 16mo, 222 pages.

Sanford, H. L.: An Efficiency Test of Dispensary Treatment of 100

Cases of Gonorrhea, Cleveland Med. Jour., 1913, xii, 813-824. 


\section{CHAPTER VII}

\section{DISEASES OF THE URETHRA (Continued)}

Stricture.-A band of scar tissue in the urethral mucosa and submucosa extending around the urethra is a stricture. So-called "stricture" of the posterior urethra is always congenital. During development a barrier of mucous membrane grows across the canal; the obstruction, therefore, is formed of normal rather than of scar tissue. "Contracture" of the prostate is virtually a stricture, except that the contraction takes place in the prostatic tissue outside the urethra rather than in the mucosa itself.

Stricture of the anterior urethra is commonly due to an antecedent gonorrhea and is formed by the contraction which follows the cicatrization of inflammatory deposits in the urethral submucosa. This process often takes place about an infected urethral gland, and is probably continuous with the infection which preceded it, beginning as the infection subsides, but not giving symptoms until a considerably later date. I doubt if stricture ever follows a urethritis which has been entirely cleared up.

Less frequently stricture follows injury to the urethra, such as is caused by a blow on the perineum. Partial or complete rupture of the urethra occurs, with the consequent deposition of exudate in its wall. The exudate undergoes 
cicatrization, and contracts much more rapidly than do the strictures of purely infectious origin. Tuberculous strictures, though rather rare, must be borne in mind. They are found occasionally accompanying tuberculosis of the bladder.

Strictures may be narrow or broad, hard as cartilage or soft and vascular, single or multiple. If multiple, the tightest one is always nearest the bladder. Behind a stricture the urethra is dilated and infected; the impact of the urinary stream keeps up the infection, and the infection adds new exudate for cicatrization. So the process continues until obstruction to the outflow of urine causes hypertrophy of the bladder and dilatation of the ureters and kidney pelves. Meanwhile the infection extends back to the prostate, the bladder, and the kidneys. The constant infection of the prostate, if begun before the age of benign enlargement, results in the small, fibrous prostate and appears to prevent the formation of adenomata. A study of 50 men fifty-five years of age or older who had strictures showed but one in whom there was any appreciable enlargement of the prostate. The infection of the kidneys plus the back pressure destroys the renal parenchyma. The avoidance of renal destruction furnishes the real reason for early and efficient treatment of stricture of the urethra.

The symptoms of stricture are gleet, frequency of urination, and difficulty in expressing the urine. In tight strictures the stream is small and has no force, but dribbles from the penis in a succession of drops. Congestion of the urethra brought on by sexual excitement, alcohol, or exposure to cold 
causes the stricture to swell, and an acute retention may result.

The diagnosis of stricture should include a knowledge of the situation and size of each contraction ring. These facts may be learned by the use of bougies à boule-acorn-tipped instruments with slender shafts made of metal or of weblbing. The largest bougie which the meatus will admit is passed until it meets an obstruction. A smaller bougie is then passed through the first constriction, down to one through which it, in turn, will not pass. In this way the location and caliber of each stricture can be ascertained, for as the bougie passes through the band its "jump" gives an unmistakable sensation to the guiding hand. If the bougie is held up at a point just in front of the cut-off, so that one camnot be sure whether the obstruction is lue to stricture or not, the passage of a fair-sized sound will give the answer. Stricture grips the sound. The sphincter does not.

Treatment.-The man with a stricture often does not have it treated until acute retention overtakes him. If such is the case, a prolonged hot sitz-bath may enable the patient to void. If it does not, the passage of a filiform bougie will open the canal temporarily. In passing a difficult stricture three or four filiforms should be passed to the point of olstruction, and manipulated in turn. False passages will become filled by several of them and one of the others will enter the proper passage. Once a bougie is passed in a difficult case, it should be fastened in with adhesive plaster strips. The urine will find its way out beside the instrument, and the 
pressure of the bougie will dilate the urethra. A very convenient instrument consists of a filiform to which a woven catheter may be screwed. Once the filiform is in, the catheter may be made to follow, while the filiform coils up in the bladder.

For a few days the urethra will remain patent, or at least partially so. After the reaction caused by the passage of the bougie has subsided (three to five days) bougies should again be passed; this should be done every five to seven days. If the stricture is pliable, one should be able to advance at least one size (French scale) at each treatment. When the stricture is dilated to about 20 French, sounds should be substituted for bougies. The dilatation must be continued steadily until a 29 or 30 French sound passes without being gripped. Too rapid dilatation, the sign of which is bleeding, does more harm than good. The newly traumatized area becomes infected and more contraction results.

The resilient or gristly stricture may resist all attempts at dilatation. It will then have to be cut. If situated at or in front of the penoscrotal angle, internal urethrotomy will suffice and may be done under local anesthesia.

The anterior urethra is filled with 4 per cent. novocain solution. The Otis urethrotome, an instrument which dilates as a screw in the handle is turned and which has a concealed knife at the tip, is passed through the stricture and dilated until the stricture is stretched tight. The knife blade is then withdrawn through the stricture. The urethra behind the stricture, being of greater caliber, is not cut. 
When the knife meets the fibrous band of the stricture it divides it. The incision should be made on the roof of the urethra, and the process should be repeated until a 3 I or 32 French sound passes easily. An indwelling catheter should be left in the urethra for two or three days. The

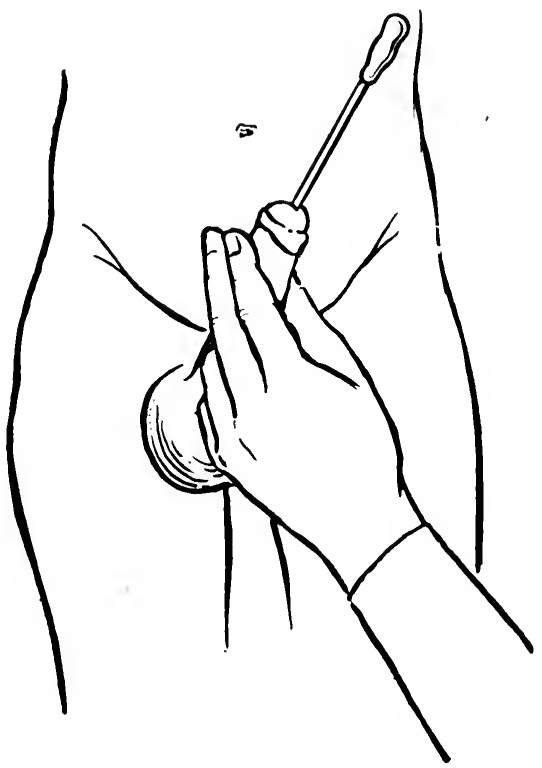

Fig. 18.-Sound is introduced with shaft parallel to Poupart's ligament. The penis is threaded on to the sound until the point of the sound can be felt in the perineum.

patient should remain in bed for several days, as hemorrhage and infection do occasionally occur.

Strictures in the bulb must be cut through the perineum, and the bladder drained for four or five days by a perineal tube. These strictures are, of course, divided on the floor 
of the urethra. The operation is simple enough if a guide can be passed through the urethra previous to operation. In impassable strictures, especially when the perineum is infiltrated and perhaps gangrenous, identification of the posterior portion of the urethra mav be very difficult. Rarely

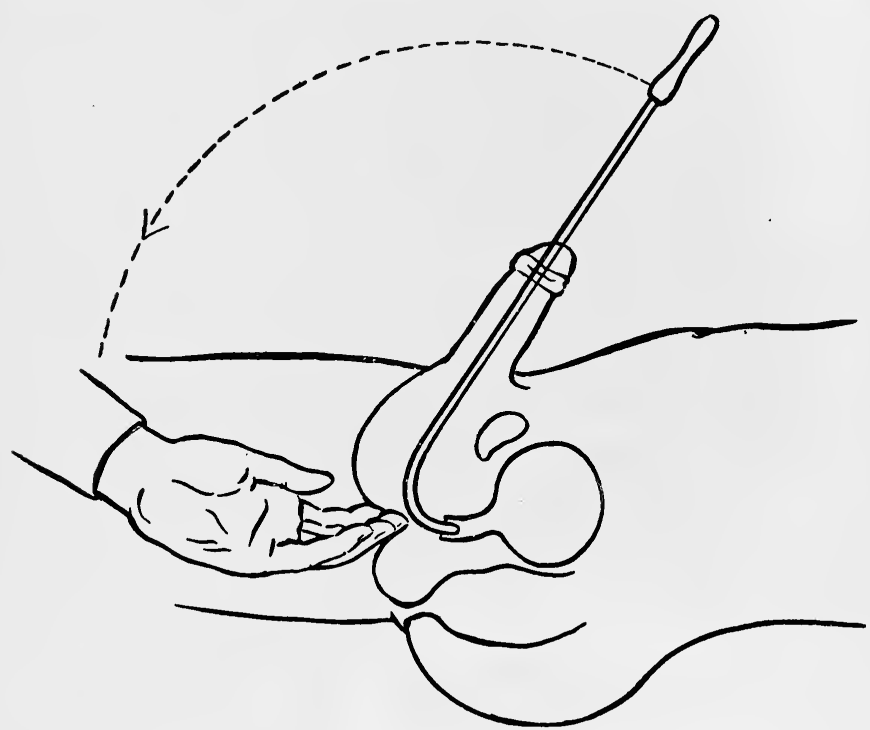

Fig. 19.-Handle of sound is swung to middle line and elevated by one hand. The first two fingers of the other hand press upon the perineum to guide the point of the sound through the cut-off muscle.

it is necessary to open the bladder above the pubes, and do a retrograde catheterization in order to locate the canal. When the posterior segment is found it should be dilated until the finger can be passed into the bladder. This dilates the bladder orifice, which is often narrowed from the cicatrization of long-continued infection. Sounds should be 
pussed from seven to ten days after operation, and as frequently thereafter as is necessary to keep the urethra at a caliber equal to 29 or 30 (French scale).

Once a stricture is dilated, whether with or without operation makes no difference, it must be held at that caliber by the occasional passage of sounds. At first they should be passed once a week. As time goes on this may be done less

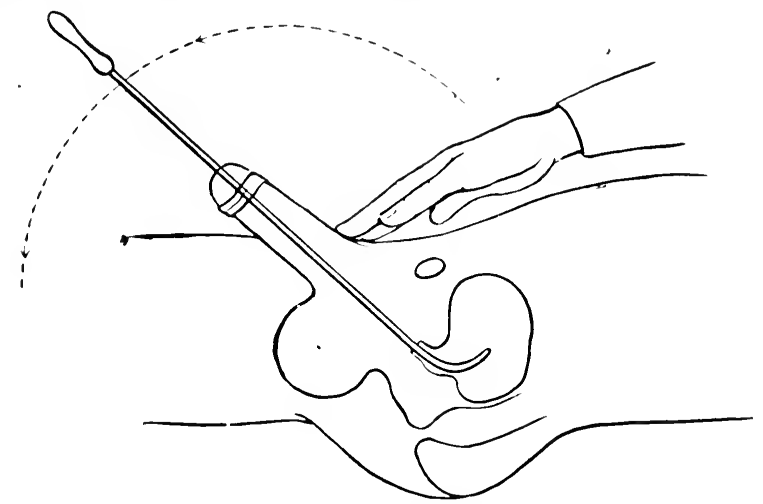

Fig. 20.- - Iandle of sound is carried downward along the line of an are, of which the cut-off muscle is the center. Ifter the point hats patserd the cut off, the hand which previously held the sound presses downward upon the suspensory ligament, while the other hand guides the sound. The handle is depressed until the sound can be felt free in the bladder.

frequently, but to be insured against the recurrence of his stricture a man must have sounds passed once or twice a year throughout his life.

Extravasation of Urine.-Occasionally as a result of obstructed drainage, but more often because of rough instrumentation, so-called "extravasation of urine" occurs. There is a brawny swelling of the peri-urethral tissues, beginning 
in the bulb and spreading over the scrotum and on to the abdominal wall. The penis becomes huge with edema, and unless drainage of the most thorough sort is provided patches of gangrene develop. It seems clear that the condition is not due to the leakage of urine alone, for that has often been shown to be comparatively harmless. The malignancy of the process is due to infection by a particular kind of anaërobe. Immediate section of the stricture and complete drainage of the infiltrated tissues must be done. Patients in this condition are often in wretched shape. In such, spinal anesthesia is the anesthetic of election.

In the management of stricture one should observe several rules:

I. Do not make a stricture bleed. Bleeding means either that a false passage has been made, or that greater reaction and contraction will follow the traumatization of the urethra.

2. For passing or dilating strictures of less than 20 French caliber never employ a metal instrument except as follower attached to a filiform guide. The point of a small sound is very easily poked through the urethral wall.

3. Always wash the urethra and, if possible, the bladder after instrumentation.

Савот, H.: Treatment of Stricture of the Bulbar Portion of the Urethra by Resection, Partial or Complete, Boston Med. and Surg. Jour., 1909, clxi, 848-850.

Савот, H., and Sмrтh, G. G.: The Influence of Stricture of the Urethra on the Development of Hypertrophic Changes in the Prostate, The Lancet-Clinic, April 27, 1912.

Keyes, E. L., Jr.: Prognosis of Urethral Stricture, Trans. Amer. Assoc., Genito.-urin. Surg., 1915, x, 11-37. 


\section{CHAPTER VIII}

\section{DISEASES OF THE URETHRA IN WOMEN}

Acute urethritis in women is ordinarily only one manifestation of infection by the gonococcus. As a rule the process clears up quickly, much sooner, in fact, than the involvement of the cervix which accompanies it: The onset of the urethritis is attended by burning urination. The inflammation may involve the trigone of the bladder, causing turbidity of the urine, but the bladder, as a whole, is not affected. Under forced fluids and sandalwood oil the process rapidly quiets down; leaving perhaps a subacute urethritis. The cure of this will be hastened by instillations of argyrol, ro per cent. strength, into the urethra, by dilatation of the urethra with Hank's cervical dilators, and by irrigation of the bladder with potassium permanganate.

Not infrequently the gonococcus gets into Skene's glands, situated one on either side of the urethral meatus. Pressure upon the anterior vaginal wall expresses the pus with which they are filled. To clean out the infection a few drops of 5 or ro per cent. silver nitrate solution should be injected into their depths by means of a I- or 2-c.c. syringe tipped with a needle the point of which has been filed off square.

Chronic Urethritis. - A not inconsiderable number of women who complain of frequent and burning urination are treated 


\section{IO4 AN OUTLINE OF GENITO-URINARY SURGERY}

for cystitis, despite the fact that the urine is absolutely negative and the bladder, on cystoscopic examination, perfectly normal except for undue redness about the trigone and vesical orifice. To treat these cases by bladder lavage is to miss the point entirely. The urethra is the seat of the trouble. The caliber of the urethra should be measured with bougies à boule or Hank's dilators in order to rule out stricture. The urethra should be palpated upon a dilator, to determine whether there is thickening of the peri-urethral glands. Examination of the urethra with the straight electrically lighted endoscope shows a congested mucous membrane, streaked with deeper red. Some of the patches of red may be almost granular and bleed easily. This examination is best made with the patient in the knee-chest position. The bladder should be thoroughly. emptied of urine, by the catheter if necessary, as urine running into the endoscope spoils the view.

For this condition the application of ro per cent. silver nitrate to the entire urethral lining by means of cottontipped applicators gives excellent results. The treatment should be given not oftener than once a week, and it is a good plan to substitute for every third treatment of this kind a simple dilatation and irrigation.

These cases of chronic urethritis seldom follow a gonococcus infection. I have seen one which was very evidently the last vestige of a pyelitis. They occur most frequently in unmarried women, and often at about the time of the menopause. Unquestionably improvement in the general condi- 
tion of the patient will improve the urethritis. H. D). Furniss has described such a case which was helped very little by local treatment, but which seemed to recover entirely following tonsillectomy.

The great relicf which can sometimes be given to such cases is illustrated by the history of Miss 1. kel.., age twenty-ix, who had been at Rutland Sanatorium for pulmonary tuberculosis. She came to the Outpatient Department of the Massachusetts (ieneral IJospitat complaining of great frequency, burning, and such urgency that unless she could find a toilet immediately she would lose cont rol of the urine. We expected to find her a case of urinary tulereulesis, but were surprised to find the urine absolutely normal. (ystosopy showed a normal bladder. The urethrat, however, was as red as a piece of raw becf. Ifter about ten alpplications of silver nitrate she considered herseff so weil that, some two years ator), she stopped treatment altogether. I have seen her at intervats since; the improvement has been permanent, and exeept during her periods she has no urinary symptoms at att.

Stricture of the Urethra.--Although not frequent, stricture in women is by no means rare. As in man, it follows an antecedent infection. The symptoms are those of urethral irritation, i.e., frequency and burning. The diagnosis is made by the passage of bougies à boule, sounds, or dilators. The treatment consists in gradual dilatation, which gives very satisfactory results.

A caruncle is a bright red, elevated papilla situated at the urethral orifice, nearly always on the vaginal sicle. Frequently it is exquisitely sensitive; half of the cases studied by Young had frequent, painful urination. Four types have been described-the angiomatous, the granulomatous, the papillomatous, and the epitheliomatous. The last group, on microscopic examination, presents the picture of epithelial 
cancer. As cancer of the female urethra is rare, while caruncle is very common, it would seem that this resemblance is apparent rather than real.

Caruncle is best treated by removal, either by cauterization, fulguration, or excision. Local anesthesia-a few drops of novocain injected beneath the little tumor-suffices. About a third of the cases removed, whether by cautery or by the knife, recur.

Prolapse of the urethra is most likely to occur in elderly women. A ring of redundant urethral mucosa surrounds the orifice. The exposed surface may ulcerate or may become very edematous. The best treatment consists of excision of the collar of prolapsed membrane, with suture of the cut edges.

Urinary incontinence, if not due to a nerve lesion, pelvic prolapse, severe cystitis, or overflow bladder, is due to a laxity of the urethral sphincter. This condition may be remedied by an operation which brings together beneath the bladder neck enough fibers of the detrusor muscle of the bladder wall to form a sort of buttress beneath the trigone and the internal urethral orifice.

Bugbee, H. G.: Frequency of Urination in Women, Jour. Amer. Med. Assoc., lxviii, 693-699.

Shallenberger: Chronic Urethritis in Women; Diagnosis and Treatment in Obscure Urethral Pain, Jour. Amer. Med. Assoc., 1916, lxvi, 1011.

Young, E. L., JR.: Urethral Caruncle, Boston Med. and Surg. Jour., clxxii, 822-824. 


\section{CHAPTER IX}

\section{INFECTIONS OF THE PROSTATE AND SEMINAL VESICLES}

Acute infections of prostate and vesicles are practically always the result of invasion of these structures by the gonococcus. Prostatic abscess due to the colon bacillus does occur, but is infrequent compared with the infections for which the gonococcus is responsible.

The prostate may be likened to a sponge into the interior of which gonococci have penetrated and have set up little foci of suppuration. Each little abscess is surrounded by a zone of inflammation. These indurated areas give upon palpation a hard, nobby sensation. The prostate is somewhat enlarged, and its chief characteristic is an absence of the soft elasticity of the uninfected gland.

Infection of prostate and vesicles takes place in more than half the cases of acute gonorrhea. In most of these the process never becomes acute enough to give symptoms; it is evidenced only by the persistence of urethral infection, by the abnormal findings on rectal examination, and by the presence of pus-cells in the prostatic secretion expressed by massage.

In a few cases the infection runs a more virulent course. The gland becomes swollen, hot, and very tender. There is 


\section{IO $\overline{8}$ AN OUTLINE OF GENITO-URINARY SURGERY}

frequency and urgency of urination, and a general febrile reaction. With rest in bed and hot rectal irrigations the process either quiets down or goes on to abscess formation. Usually it follows the former course; less frequently, the

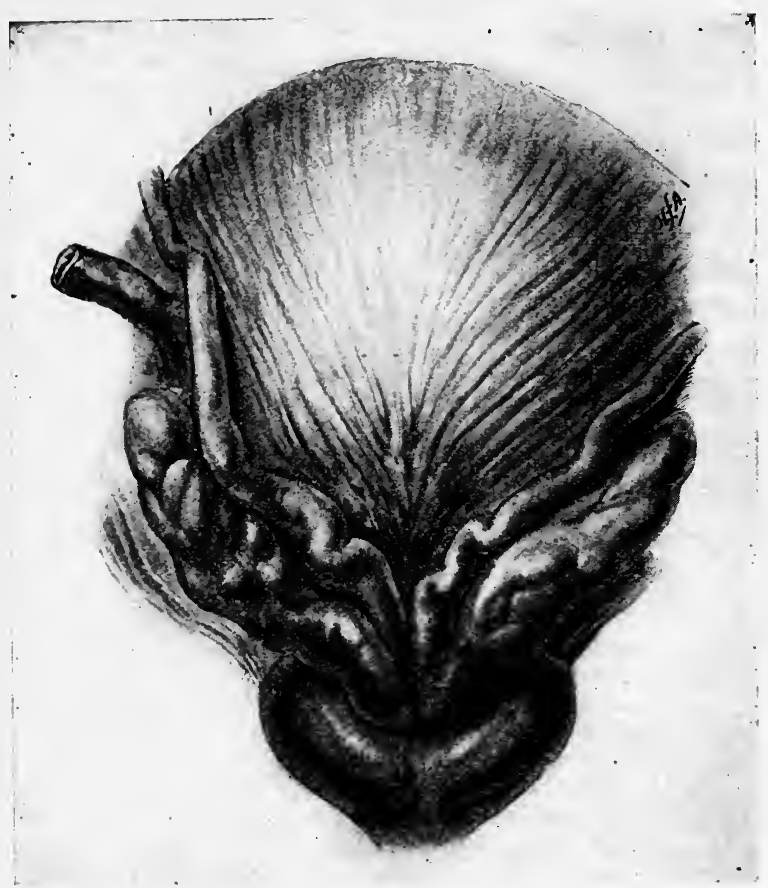

Fig. 21.-Dissection of the base of the bladder, showing prostate, seminal vesicles, vasa deferentia, and one ureter.

latter. Even if a definite abscess does form, operation is rarely necessary, as the majority of prostatic abscesses rupture spontaneously through the urethra or through the rectum. If rupture does not speedily occur, with marked 
relief of symptoms, the abscess should be drained. A preanal incision, through which the posterior aspect of the prostate may be reached without disturbing the cut-off muscle, is the best method of approach.

Acute infections of the seminal vesicles, like those of the prostate, are almost always due to the gonocoecus. Edema

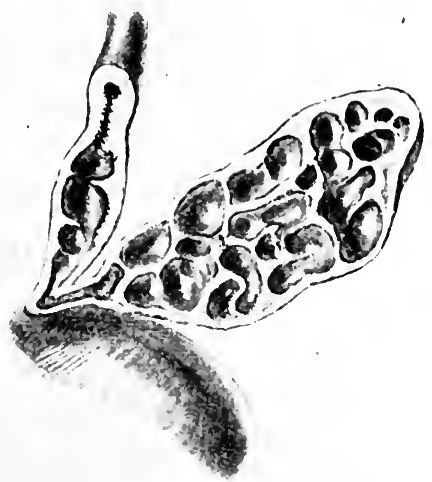

Fig. 22.-Seminal resicle and ampulla of the vas deforens "th the

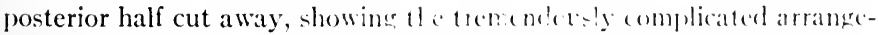
ment of the interior. The alsence of drainage facilities explains why infection persists so long in the vesicles.

of the mucosa shuts the ejaculatory ducts; the infected secretion backs up into the resicles and there sets up a marked reaction. Studies of the seminal vesicles by Pickel have shown that in the majority of cases they consist of complex, convoluted tubes, the mucous membrane of which is full of glands and crypts (B. A. Thomas). Great opportunities are offered infecting organisms to form foci in these crypts. In 


\section{IIO \\ AN OUTLINE OF GENITO-URINARY SURGERY}

the majority of cases the eradication of infection is made difficult by the presence of diverticula given off from the main tubule. Pockets are formed, the drainage of which through natural channels is very insufficient. Into this maze of crypts, pockets, and blind by-ways the gonococcus penetrates and forms small foci of suppuration. Inflammatory reaction about these foci causes thickening of the wall of the vesicle. Thus a stiff-walled, infected cavity is formed, a condition which in no way helps in clearing up the infection.

Acute infection of the vesicles may cause pain in the groin and backache because of distention of the sacs by pus and retained secretion. Occasionally the vesicles rupture, with the escape of pus into the perivesicular tissue. The abscess, kept from the rectum by the sheet of fascia (Dénonvillier's fascia) which separates prostate and vesicles from the rectum, may point in the perineum.

P. N. H. Married, aged twenty-nine. Gonorrhea eleven years ago, apparently cured. Married two years later, wife never pregnant. After marriage his discharge reappeared. His wife had an "abscess about the womb." Four weeks ago he had extramarital intercourse, followed in one week by a urethral discharge. For two days he has had difficulty in voiding, and the rectum has been very sore. Examination showed a profuse urethral discharge which contained gonococci. No stricture found. Testes and epididymes normal. By rectum, left side of prostate showed much induration and tenderness. The urine was hazy with pus and showed a slightest possible trace of albumin.

He was put to bed and given daily rectal injections of hot water. The condition of the prostate and vesicles became worse, and after ten days he was sent to the hospital. There he was kept under observation for a week or more, now better and again worse. He ran an evening temperature of 2 or 3 degrees elevation, and an area of induration began to develop in the perineum. He had a great deal of pain. The urine had become clear. Operation was decided upon. Under spinal anesthesia a 
curved preanal incision was made. The rectum was dissected back and an abscess containing several drams of pus was opened. The abscess cavity extended upward to the left seminal vesicle. The dissection was carried upward until the vesicles were exposed. The left vesicle was identified and freely incised, with the escape of thick pus. The right vesicle was not identified. $\Lambda$ rubber tissue wick was placed over the vesicle and the wound closed.

Operation was followed by immediate relief of pain and fall of temperature. The wick was removed at the end of a week and the wound healed quickly. He returned to work in New Hampshire and was seen occasionally. Two and a half months later examination showed clear urine, no urethral discharge. Prostate and vesicles felt" matted together. The secretion expressed by massage showed spermatozoa and no pus at all. Complement-fixation test was negative. Having divorced his wife, he was about to be married again.

Invasion of the blood by the gonococcus may take place, accompanied by fever and perhaps causing metastatic growths in endocardium or joints. Septicemia, as evidenced by endocarditis and more frequently by arthritis, is not a common complication. The former manifestation may often escape observation, but, so far as is known, it is not at all frequent. Arthritis of greater or less severity occurs chiefly among the cases treated improperly or not at all, and in my experience is nearly always due to an infection of the vesicles.

Arthritis of gonococcus origin is usually polyarticular. Fleeting pains may be felt in a number of joints, but the process finally settles in only a few of these. The small joints of the feet are especially prone to become involved, and the knee is perhaps the next most frequent. The process affects the synovial surfaces, and has the same tendency to form fibrous tissue that it has in the urethra.

Gonococcus arthritis may be divided into two forms-the 


\section{2 AN OUTLINE OF GENITO-URINARY SURGERY}

very acuite, fulminating type, in which the joint becomes swollen, red, exquisitely tender, and later suppurates, and the more chronic type, in which the process comes on less abruptly, and consists of peri-articular swelling without any of the signs of acute inflammation. In the first type, immediate opening of the joint, washing it out with salt solution or sterile water, and closing it tight with immobilization, is the only way to save the motion of the joint. For the less acute type, complete rest at first, followed later by passive, then active, motion.

In addition to treatment of the joints, treatment directed against the focus from which the infection is derived is a necessity. This usually consists in regular massage of the prostate and vesicles. Not only does this clean up the focus, but it seems to give immediate results through the vaccination of the patient with his own organisms. Massage, in other words, liberates gonococci or their toxins, which increase the bodily resistance to the infection. To intensify this action a course of six injections of gonococcus vaccines may be given. The dose should be as follows:

$500,000,000$ the first day.

$750,000,000$ the third day.

I, $, 00, \infty 00, \infty 00$ the sixth day.

I,250,000,000 the ninth day.

I,500,000,000 the twelfth and fifteenth days.

If the arthritis does not improve under these measures, the question of draining the seminal vesicles by the operation of vesiculotomy should be considered. 
Seminal vesiculotomy has been advocated for this condition. A few genito-urinary surgeons are enthusiastic advocates of the operation. Unquestionably, there are cases in which prompt drainage of the vesicles gives much speedier and more complete results than any other method of treatment. The operation is not without risk. It may be attended by considerable shock. One case operated by me died a week later, apparently from gonococcus septicemia. In my opinion the operation should be reserved for (I) cases of very acute vesiculitis in which the ordinary methods fail to give relief; (2) cases of gonococcus arthritis which show no definite improvement after massage of the vesicles has been faithfully carried out for two or three months.

Medication of the vesicles by the injection through the lumen of the vas deferens of some germicide, such as argyrol, is not without advocates. Under local anesthesia a small incision is made in the scrotum. The spermatic cord is pressed into the incision and the vas is isolated and opened. The needle of a ro-c.c. syringe is passed into the canal toward the body and the solution to be used is injected. Except by a few genito-urinary surgeons the method has not been generally accepted.

Vaccines are employed quite extensively in the above conditions, as well as in other forms of gonococcus infection. It is hard to judge accurately their true value. My own experience with them has not convinced me that they are really helpful except in arthritic cases. If employed at all, they should be used simply to increase the patient's resist- 
ance to the invading organism, and not as a substitute for local treatment.

So much for acute infections of prostate and vesicles; they occur with relative infrequency, considering the vast number of infections of these organs which occur every year. In managing such cases one should pursue an expectant policy until it is clear that there is an abscess which shows no signs of spontaneous drainage.

Subacute and Chronic Prostatitis and Vesiculitis.-In the great majority of men infected by the gonococcus the infection involves the prostate or vesicles, or both. Herbst says: "In over 75 per cent. of cases in which the gonococcus invades the male urethra it travels back of the cut-off muscle and into the posterior urethra, and in most, if not all, of them the ejaculatory ducts and subsequently the seminal vesicles become involved."

It is my own belief, the correctness of which is not easy to prove, that infections of the prostate quickly clear up of their own accord. Infections which persist, though often called prostatic, are really of the vesicles. In comparatively few cases is this posterior infection severe enough to cause symptoms troublesome to the patient. In most, a cloudy second urine, with perhaps some frequency for a day or two, are the only outward signs of the spread of the infection. In many of these cases the urethritis clears up completely. In others it gradually diminishes and persists as a slight dampness at the meatus, accompanied by an uneasy feeling on the part of the patient that there is still something wrong. 
If patients of either type come into the physician's hands, examination of the prostate and vesicles should be made. No case of gonococcus urethritis, no matter how trivial, should be discharged without an examination of the prostatic and vesicular secretion. If the patient has been getting posterior irrigations, the bladder should be filled with antiseptic solution. If his entire trouble has apparently been anterior, the examination may be made with the bladder full of urine. The examining finger, encased in a well-lubricated finger-cot, is inserted through the anus. This is made easier if a little lubricant is smeared about the outside and the buttocks are separated by the fingers of the left hand. 'The patient bends forward over a table or chair and the right forefinger is inserted slowly. Directly beneath the finger, separated from the anal sphincter by about $\frac{1}{2}$ inch of soft tissue, lies the prostate. Only by experience do we learn to distinguish the normal from the abnormal gland. There are two types of normal prostate-the small, soft kind, and the large, elastic kind. If the lobes are hard to the touch or feel shotty, the gland is almost certainly infected. Such a prostate may remain in the same condition for years, with no sign of trouble in the urine. The induration is due to areas of scar tissue, the result of focal infection.

The finger should easily reach the upper border of the prostate. Above, extending upward and outward close to the pelvic wall, lic the seminal vesicles. These may be normal even if they are palpable; if such is the case, they are soft and one can feel the movement of the fluid within them as the 


\section{II6 AN OUTLINE OF GENITO-URINARY SURGERY}

finger presses down. If they are infected, as they are in a large majority of cases, they feel thickened, or contain indurated masses, or are plastered on to the pelvic wall in such a way that they form a shelf from side to side.

By very gently but persistently stroking the vesicles from above downward, and by rubbing more firmly upon the prostate, a few drops of secretion may be expressed from the mouth of the penis. This is examined for pus, as described in Chapter II.

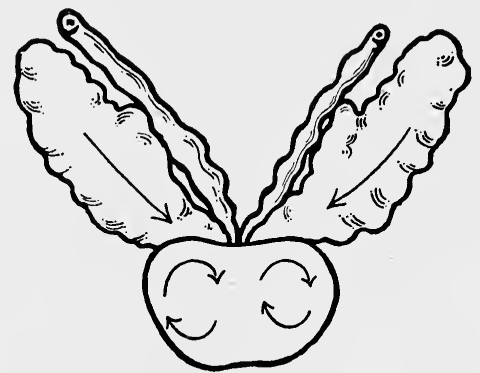

Fig. 23.--Diagram of prostate and vesicles. Arrows indicate direction of massage.

Examination of this secretion for gonococci is really a useless task. Even if present, they are few in number and probably more or less atypical in form, so that their recognition is not certain. If search is negative, one cannot be sure that the infection has disappeared. The specimen of secretion on the slide represents so small a portion of the whole that its testimony on this point is not valuable.

The presence. of pus-cells in any considerable number means that the posterior structures are, or have been, in- 
fected. In the secretion from normal prostates rare pus-cells may occur, but the presence of more than one cell in every four or five high-power fields indicates an inflammatory process.

The inflammatory process so indicated may be due to the recent infection, or may have followed a previous infection by the gonococcus or by other organisms. One must consider the history of the case in this connection. The history of a previous gonorrhea, since the inception of which there has always been a little discharge, strongly suggests that the prostatic condition is a relic thereof. A perfectly negative past history, with a present attack of urethritis, makes it equally probable that the pus in the prostate is due to the current gonorrhea.

In either case the existence of prostatic or vesicular infection coexistent with or following a gonococcus urethritis constitutes a definite indication for the treatment of prostate and vesicles.

L. A. C., aged twenty-nine, came to me on $\mathbf{1 1 a r c h} 30,1915$. He wished to marry, but feared to do so because of a persistent gleet. Cionorrhea twice, last time in 1910. This attack lasted about three months. He had never had massage of prostate. Since 1910 he has had a slight discharge. The urine is clear. Except for a varicocele, external genitals are normal. The prostate on rectal palpation is large and soft, the vesicles thick and indurated. Much secretion expressed, showing some pus and spermatozoa. Complement-fixation test "moderately positive." He was treated once a week for two years; massage was the chief treatment, although full-sized sounds were frequently passed. In November, 1916, the complement-fixation test was negative. January 10,1917, my notes read, "No discharge now. Vesicles feel hard and sclerosed, prostate much smaller than at first. Secretion expressed by massage shows only a few pus-cells." He was allowed to marry. 
Chronic Non-specific Prostatitis and Vesiculitis.-Another type of infection of prostate and vesicles is non-specific in character. This may be the result of a gonococcus infection from which all the gonococci have died out, or may occur in individuals who have never had gonorrhea. Chronic prostatitis and vesiculitis of non-specific origin are by no means rare, as they give the same symptoms and require the same treatment as the prostatitis occurring as a late sequel to gonorrhea. The two will be described together.

The symptoms are of two kinds-sexual and urinary. The sexual symptoms take the form of irritability of the sexual reflex-i.e., premature ejaculation and nocturnal emissions, or at a more advanced stage, of sexual impotence. There will be inability to have an erection, associated very probably with an increased mental appetite for intercourse. The urinary symptoms consist of frequent desire to urinate, urgency, and burning micturition.

C. W. H. Aged twenty-four. Single. First seen January 24, 1916. Complaint: Occasional attacks of frequent, burning urination. Premature ejaculation. Slight gleet. Denies venereal infection. His trouble began two years ago, when, although he had not been exposed, he developed a slight grayish urethral discharge which lasted some six weeks. Examination showed clear urine, no sugar or albumin. Sediment: a few pus-cells. No stricture. Meatus size 22 French. Prostate soft. Vesicles cord-like and knotted, extending across the rectum like a hammock. Massage brought forth much flaky detritus, which showed rare spermatozoa and no pus. Meatotomy was done, after which the posterior urethra was dilated with sounds and the Kollmann dilator. The vesicles were massaged once a week, and great quantities of secretion were expressed. In three months his symptoms were definitely relieved and he stopped treatment. 
This seemed to me a case of long-standing non-specific vesiculitis. The vesicles showed the end-results of infection, but required the stimulus of massage to enable them to clear themselves of inspissated secretion. There was a contracture of the bladder neck, due to the same process.

Both sexual and urinary symptoms are due to the effect which long-continued infection of prostate or vesicles has had upon the mucous membrane of the posterior urethra, and particularly of that which covers the verumontanum. Consequently, treatment must be directed toward removing that cause-the prostatitis or vesiculitis-by massage, and also to rejuvenating the urethral epithelium.

Twice a week, every five days, or once a week, according to economic and pathologic conditions, the patient must present himself for massage. All cases cannot be treated alike; here, again, one must tread gently and avoid making a bad matter worse. One must keep in mind the thing he wishes to accomplish, and must with every treatment ask himself what he is trying to do. Massage expresses from such ducts as are open the retained pathologic secretions; it also increases the blood-supply to areas of exudate, thereby hastening the absorption of the inflammatory tissue. If these areas are rudely broken up, or if septic products pent up in closed cavities are squeezed too vigorously, the infection is spread.

Rough massage is followed in a few days by greater induration and by signs of infection in the urethra; gentle, persistent rubbing, which should not hurt the patient, is followed by gradual disappearance of the exudate and by the resump- 


\section{0 \\ AN OUTLINE OF GENITO-URINARY SURGERY}

tion on the part of the prostatic tissue of a natural elasticity. When starting such a course of treatment the patient should be told that it may extend over a period of from six months to two years. The product of years of infection cannot be removed in a couple of months.

In regard to the urethral epithelium nothing seems to work better than silver nitrate. It may be instilled into the posterior urethra through the Keyes instillator-I5 drops of Io per cent. silver nitrate at a time-or may be applied by an applicator through an endoscope directly to the verumontanum. In my hands the former method has proved the more satisfactory.

The urinary symptoms also may be caused by a stiffness of the bladder neck, due to infiltration, which allows the urine to trickle into the posterior urethra and excite the urinary reflex. For this condition one must practice dilatation with sounds or with the Kollmann dilator.

These three measures, therefore-dilatation, stimulation with silver nitrate, and massage - are to be relied upon in the treatment of chronic prostatitis and vesiculitis. For every case there is a particular combination which gives the best results, and by cautious experimentation this combination must be discovered.

Cure of Gonococcus Infections.-Patients often ask their doctor "Are you positive I can be cured?" It is right to answer "Yes." Every gonococcus infection, at least in the male, can be cured, provided the patient will come for treatment as often and as long as necessary. It is not always 
possible to remove all after-effects. Stricture may be incurable, sclerosed vesicles cannot be given back their elasticity, and scarred prostates containing foci of infection cannot be guaranteed against outbreaks of a non-specific nature later in life. The gonococcus can always be eradicated. The unfortunate sequelæ can usually be prevented; if they do result, they can be kept under control by occasional treatment.

The tests of cure vary according to the nature of the infection. In simple anterior urethritis the criteria of cure are: absence of all discharge; perfectly clear urine, free from shreds; absence of infiltrated peri-urethral glands (determined by palpation of the urethra upon a sound); a soft prostate and vesicles with no pus in the expressed secretion.

The complement-fixation test is of no great value here, as it may not be affected at all by an anterior urethritis.

The question of cure in cases in which the prostate or vesicles have been involved is much less simple. In those favored individuals whose prostate and vesicles have not been shot through with infection, a short course of massage will suffice to restore to the infected parts their normal elasticity, and to clear their secretion of pus-cells. Such patients should be kept under observation for a couple of months, the urine watched for the reappearance of shreds, and the prostatic secretion examined several times for pus. If these conditions remain negative without treatment after the patient has returned to his usual habits, no further proof of cure is required. 


\section{AN OUTLINE OF GENITO-URINARY SURGERY}

In many cases the question of cure is much more complex. These are the cases in whose prostates and vesicles such inflammatory changes have taken place that the restoration to normal is out of the question. After a year or two of massage the greater part of the exudate which at first plastered vesicles and prostate together in one mass may have been absorbed. Subjective symptoms have been relieved and discharge checked, but the secretion still contains pus, and the prostate and vesicles still feel scarred and thickened. In cases such as these one must rely to a large extent upon the intuition which he has gained through experience. Failure to grow gonococci from the expressed secretion has some value. Their absence from smears is inconclusive. Lack of a fresh outbreak of urethritis upon provocation by drinking and by the application of silver nitrate to the urethra suggests that the infection has died out. Approximate return to normal upon palpation points to a cure. If the prostate and vesicles are left alone for three months and at the end of that time show improvement rather than relapse, one may feel encouraged. All these data, taken together and weighed judicially, justify an experienced observer in declaring a cure. His opinion may then be checked up by the complement-fixation test. If the test is definitely negative, he may rest easy in his decision. If it is definitely positive, he had better wait another three months before allowing the patient to marry. In the interim the patient should be under observation. Repeated negative findings during that period will justify a verdict of "cured." 
Tuberculosis of the prostate and vesicles is rarely primary. A few cases of primary infection of the prostate and vesicles have been reported (Koll), but their occurrence is rare. Prostate and vesicles are usually involved secondarily to tuberculosis of the epididymis, and the situation is part of the big question of genital tuberculosis.

Tuberculosis of the vesicles is usually symptomless; that of the prostate is likely to involve the mucosa of the bladder neck, and give symptoms of vesical irritability. A majority of men with even unilateral genital infection are sterile.

Removal of prostate and vesicles has been practised, but in our experience the less radical method of hygienic and tuberculin treatment, with removal of more easily accessible foci, such as the epididymes, has proved fairly satisfactory.

Belfifin, W. T.: Pus-tubes in the Male, Trans. Amer. Urolog. Assoc., 1909 , iii, 13-23.

Thomas, B. A., and Paxconst, II. K.: Observations on the Pathology, Diagnosis, and Treatment of Semina! V'esiculitis, Ann. Surg., 1914, $1 \mathrm{x}, 313-318$.

Smitil, E. ().: Anatomy and Pathology of the Seminal Vesicles, Urologic and Cutaneous Rev., 1916, xx, No. 2.

Herbst, R. H : Seminal Vesiculitis as the Cause of Persistent Urethral Discharge, Jour. Amer. Mel. Assoc., 1917, 1xviii, 761-76.3.

Geragnty, J. T.: Rôle of Seminal Vesicles in Non-gonorrheal Infections of Posterior Urethra and Bladder, Jour. Amer. Med. Assoc., 1917, Ixviii, 757.

SQuifr, J. B.: Surgery of the Seminal Vesicles, Cleveland Med. Jour., 1913, xii, 801-813.

Saxe, G. A. DeS.: The Persistence of the Conococcus in the Prostate, Trans. Amer. Urolog. Assoc., 1909, iii, 131-157.

Koll, I. S.: Primary Tuberculosis of the Prostate Gland, Ann. Surg., 1915, lxii, 473-475.

Barney, J. I.: Tuberculosis of the Epididymis: Its liffect Upon Testicle and Prostate, Boston Med. and Surg. Jour., 1913, clxviii, 923-927. 


\section{CHAPTER $\mathrm{X}$}

\section{PROSTATIC OBSTRUCTION}

IN men the common causes of obstruction at the bladder neck are nerve lesions, especially those of tabes; tumor with a ball-valve action; stone in the urethra; contracture of the bladder neck; carcinoma of the prostate, and benign enlargement of the prostate. Of these causes, the last is by far the most frequent.

Benign enlargement of the prostate, erroneously called "hypertrophy," is due to the formation within the gland of definite adenomatous masses. These consist of glandular and connective tissue, surrounded by a capsule of the latter. They can be shelled out in the same way as fibroids of the uterus, and except for the glandular element in their make-up might well be compared with uterine fibromata. The cause of their formation is unknown. The best preventive seems to be infection of the prostate a number of years before the age at which adenomata begin to form. Prostates scarred by chronic infection seem less liable to adenomatous change. These changes seldom are enough to give symptoms before the age of forty-five, and usually not before the age of fifty.

Adenomata occur in the lateral lobes, the median lobe, and occasionally in the glands of the trigone. They never 
take place in the posterior lobe, which seems to be reserved as the starting-point for cancer (Boyd and Geraghty). .

The growth of adenomata in the lateral and median lobes may form a horseshoe-shaped collar upon three sides of the bladder neck. This raises the urethral orifice above the base of the bladder, thereby preventing complete emptying. Or the lateral lobes alone may become enlarged and by jutting into the posterior urethra may hinder the outflow of urine. Occasionally a single pedunculated mass springing from the trigone or from the edge of the urethra may cause much obstruction by its ball-valve action.

Symptoms.-First Stage.-In the early stage of prostatic enlargement, before the obstruction is enough to cause trouble, there occurs a period of indefinite length characterized by congestion of the prostate. The investing mucous membrane is intensely congested and may bleed without any provocation. The patient has urinary frequency, especially at night, although he may pass but a very little urine at a time. The flow of urine may be interfered with, the act of micturition requiring a conscious effort at expulsion, and being followed by dribbling. During this stage there is likely to be a small amount of residual urine, perhaps I or 2 ounces.

The amount of congestion waxes and wanes according to the behavior and general condition of the individual. Excesses of all kinds-overfatigue, exposure to cold-affect the prostate of this type. The symptoms of irritability may disappear, but the amount of residual seldom recedes. 
Prostatic Enlargement.-First Stage.-D. P. K., a lawyer eighty-four years of age, was referred to me in February, 1917, because of urinary frequency and hematuria. He had never had urinary trouble before, except that for the past few years he had found it necessary to get up two or three times at night. He had had an attack of angina pectoris a short while before, but otherwise has always been well.

For two or three days he had been having irritability of the bladder, gradually increasing. When I saw him he was unable to control his sphincter perfectly, and there was some leakage of urine. The urine contained blood-cells, a rare cast, no pus, no sugar. Specific gravity 1020. Reaction acid. Reflexes were normal. By rectum, the prostate felt soft, elastic, not markedly enlarged. He was kept indoors, given forced fluids, a diet free from meat and spices, potassium citrate, 10 grains, three times a day, and 10 minims of sandalwood oil with each meal. His symptoms rapidly disappeared. In two days the urine became free from blood. February 24 th he was catheterized with a small soft-rubber catheter. One ounce of residuum was obtained. Although the catheter passed easily, there was slight bleeding from the bladder neck. A few days after this he again had frequency, and the urine showed blood-cells and colon bacilli. He was put on hexamethylenamin, 30 grains per day, and five days later reported that he felt as well as he had before his upset. The urine was clear, with no albumin and only a rare epithelial cell in the sediment.

Since that time (one year ago) he has been well. If the urine becomes too concentrated or he becomes chilled, urination will become frequent and his urine "hot." He rests, drinks water freely, takes a few capsules of sandalwood oil, and the symptoms disappear.

Second Stage.-Usually the residual urine increases; a larger and larger amount of urine is left in the bladder. If uninfected, the residuum may gradually reach such an amount that the bladder becomes palpable above the pubes. I have seen the bladder distended to the umbilicus without the patient's being aware of his condition. Small amounts of urine are passed frequently, but are not enough to keep ahead of the amount secreted. Such a bladder is called an "overflow bladder." The distention may increase so gradually that no distress is 
felt; the muscle-fibers of the bladder wall at first hypertrophy, later become stretched and atonic from the constant pressure. The ureters and renal pelves become dilated, the renal cortex

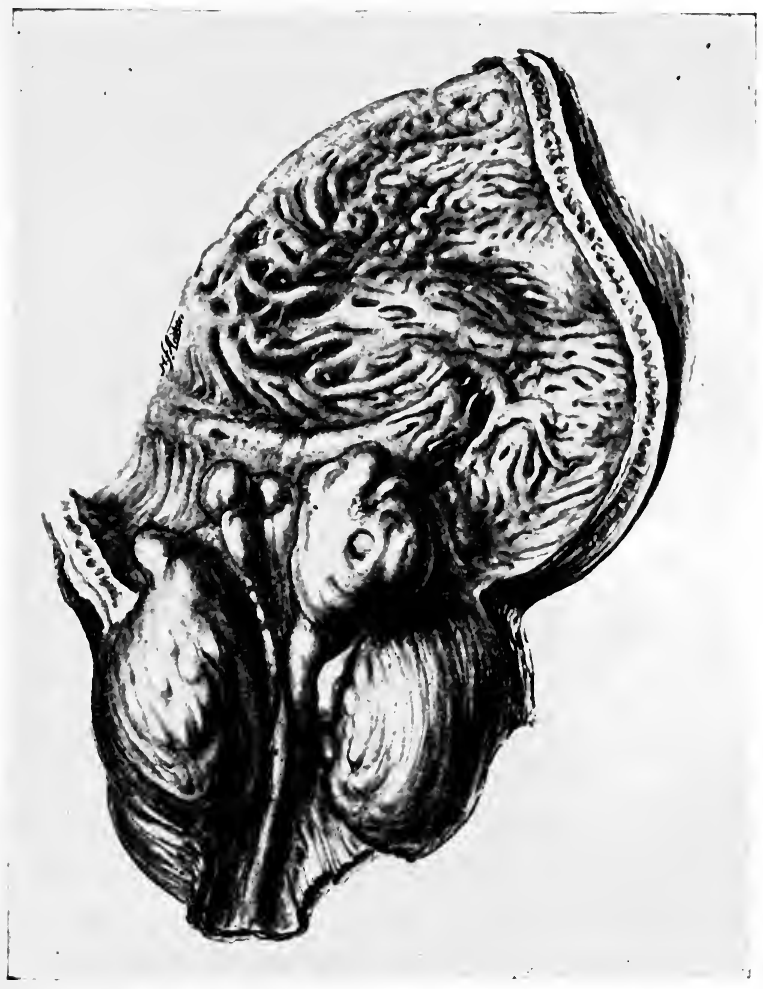

Fig. 24.-Drawing of bladder removed at autopsy, showing adenomatous changes in the prostate and resulting thickening and trabeculation of the bladder wall.

thinned. It may be that the first symptoms to attract the patient's attention will be those of uremia, manifested by digestive disturbance and loss of weight. 
In an infected bladder the residual urine seldom reaches this point without giving symptoms enough to send the man to the doctor. An infected bladder which carries a residuum of 6 ounces or more is likely to cause much frequency and rapidly increasing difficulty in urination, due to the added congestion of the gland caused by the infection. The patient shows evidence of septic absorption-loss of weight, fever, malaise, and a dry, dirty tongue, bright red at the tip and sides. Infection is easily carried up the dilated ureters to the kidneys, thereby adding to the damage already caused by back-pressure.

In cases with considerable residual urine, uninfected as well as infected, acute retention may occur at any time. Suffering then becomes intense. This is the third phase of prostatic enlargement.

The treatment of the three phases-(I) congestion, (2) partial retention, (3) complete retention-will be considered later.

Diagnosis of benign enlargement of the prostate can generally be made by a consideration of the age of the patient, his story, and the condition of the prostate as determined by rectal examination.

The age-fifty or more. If he is below forty-five, one should entertain with skepticism the theory that benign enlargement accounts for his troubles. The insidious onset of bladder irritability, especially at night, the increasing difficulty in expressing the urine, the dribbling after urination, are typical symptoms. If a clear urine accompanies these 
symptoms the difficulty will not be due to stricture. The urine of stricture always shows shreds. If one suspects stricture, it can be definitely ruled out by passing a bougie à boule to the cut-off muscle.

Upon rectal examination the prostate should feel definitely thickened. It may be of the large, smooth, rounded, elastic type, or may be divided into the two lobes and give an impression of thickness rather than actually project into the rectum. It is easy to mistake the base of a distended bladder for a very large, soft prostate. An adenomatous prostate must be separated by a sulcus from the pelvic wall. The enlargement begins about $\frac{1}{2}$ inch above the anal sphincter; if there is a hard area in the middle line extending toward the sphincter, one should suspect cancer.

The diagnosis between carcinoma and benign enlargement of the prostate is at times very difficult. The manner of onset of the trouble may be of some significance. Adenoma of the prostate comes on insidiously, beginning with nocturia: the symptoms are at first not very insistent and tend to be aggravated by such factors as cold weather and driving. The patient often says he had noticed a little trouble for quite a long time-a year or more, perhaps-before he felt the necessity of consulting a doctor. Carcinoma gives a briefer history, and its demands are more peremptory. In benign enlargement urination may be difficult, but is not, as a rule, especially painful. In cancer it is more painful, but not so difficult. Pains in the back and along the course of the great sciatic nerve are always suggestive of carcinoma. 
The real factor in the differential diagnosis is the way the prostate feels upon rectal palpation. Advanced carcinoma is easy to detect. The prostate and vesicles are of stony consistency, densely adherent to the pelvic wall. The surface is hob-nailed, with small round nodules lying upon the rectal aspect. In early cases there may be only a small area of typical stony hardness, and even this may be masked by softer overlying tissue. The most difficult differentiation lies between the small, hard prostate in which fibrous changes predominate over the adenomatous, and the malignant prostate.

Upon catheterization the adenomatous prostate shows an increase in urethral length. The catheter has to travel farther before it strikes urine. There is not a tremendous amount of pain on catheterization unless there is acute retention and much congestion. In cancer the posterior urethra is exquisitely tender, and the catheter seems gripped at that point. Bleeding is easily induced in both.

A few adenomatous prostates produce an amount of obstruction entirely out of proportion to their enlargement as determined by rectal palpation. In these cases the obstruction is usually caused by a middle lobe of the pedunculated type. Other prostates which by rectum feel huge, may hardly obstruct at all. The answer to the question of obstruction can be determined with certainty only by catheterization and measurement of the residuum.

The diagnosis between obstruction due to prostatic changes and that caused by tabes dorsalis may not be easy. In 
occasional instances both conditions exist at the same time. In the case of every suspected prostatic, examination should be made of the pupillary reaction to light, of the patellar and ankle reflexes, and of Romberg's sign (inability to stand without swaying while the eyes are shut). If these tests are all negative, the chances of the condition being due to tabes are very, very small. Yet involvement of the vesical reflex may be the only sign of tabes; this fact is illustrated by the following case:

Retention Due to Tabes Dorsalis. - Iugust 24, 1917, I was called in consultation to see I). M. II., who had been having attacks of acute retention. He was a banker, forty-seven years of age, marricd, with two healthy children. Twenty years before he had had gonorrhea and syphilis, the latter treated for one and a half years with mercury pills. Three months ago he began to have to strain to pass urine. Ilad no nocturia. On July 28 th he was catheterized and 2 quarts of urine were removed. Since then he has been catheterized a number of times at irregular intervals. During the preceding three weeks he had been catheterized four times.

Examination showed a spare, healthy appearing man. Pupils reacted and were equal. Knee-jerks were lively. Rectal palpation: small, soft prostate. Urine was clear. Residuum, 10 ounces. (ystoscopy lugust 25th showed a clean, slightly trabeculated bladder. Ureteral orifices not remarkable. Prostate not enlarged. Bladkler neck not tight. The next day the resiluum was 2 ounces. Metal catheter passed easily. Blood showed a strongly positive Wassermann.

He was carefully examined by a neurologist, who could find no ab)normality whatever in any of his reflexes. Spinal fluid showed 20 cells per cubic millimeter, increased protein content, and a strongly positive Wassermann test.

August 31 st he had no residuum and was voiding easily. He was put under the care of a neurologist and returned to his home in the West.

Tabetic retention is characterized by an insensitiveness of the bladder. Thus an infected residuum which in a pros- 


\section{I32 AN OUTLINE OF GENITO-URINARY SURGERY}

tatic would cause great frequency passes unnoticed in a tabetic. Not infrequently a tabetic presents himself with a history of incontinence. Examination shows a bladder distended beyond belief, yet not causing any discomfort at all.

Management of Prostatics.-First Stage.-Treatment during the period of prostatic irritability, before actual obstruction has developed, must be directed against the congestion of the prostate and toward the relief of symptoms. At the first examination one should be able to tell, from rectal palpation and suprapubic percussion, whether or not there is a large amount of residual urine. A residuum of more than 8 ounces gives a feeling of fulness upon rectal examination, particularly when counterpressure is made upon the lower abdomen. When the lower abdomen is percussed dulness denotes the size of the bladder. If there is no evidence of any considerable residual urine, measures should be instituted against the congestion of the prostate gland. As Keyes very aptly puts it, "The prostatic man resembles the menstruating woman in that any exposure or overdoing reacts promptly upon his pelvic organs." There must be a vigorous curtailment of daily activities. Overeating, overworking, worrying, must, as far as possible, be interdicted. Alcohol, except for an occasional small glass of wine with dinner, must be left alone. Peppery, spicy foods had better not be taken during the time when the congestion is most acute. Exposure to cold is especially to be avoided. For positive treatment one should give potassium citrate and sandalwood oil internally. The amount of water ingested should be not 
less than 80 ounces a day, and may well be more. Rectal injections of very hot water once a day are soothing, and so are hot sitz-baths. Gentle prostatic massage may be tried.

As a rule, the symptoms of irritability will quiet down to a large extent upon confinement to the house, the ingestion of plenty of water, and the use of sandalwood oil. Until they quiet down one should not attempt to determine the exact amount of residual urine. After they have subsided, however, it is best to find out how much obstruction exists. Upon the answer to this question the future management of the case depends. The residual urine is drawn with a softrubber catheter or a coudé woven catheter, passed with the utmost gentleness. The bladder should be left full of boric acid solution. If the residual urine is less than 2 ounces, and uninfected, it may be disregarded. The only indication for prostatectomy, as a rule, is mechanical obstruction; very occasionally the repeated hemorrhages may require radical measures.

Second Stage.-If the residuum is more than 2 ounces, the question of operation should be considered. A residual urine of that amount is sure to increase within a very few years. If the patient is in good condition, the likelihood of his requiring operation later may make it advisable to forestall the mishaps which may intervene, and do it now. If his years are already numbered, he may be allowed to go on, being seen occasionally and his residual urine kept track of.

The amount of obstruction caused by the prostate will 


\section{I34 AN OUTLINE OF GENITO-URINARY SURGERY}

vary somewhat with variations in the extent of congestion of the gland. When an attack of acute congestion subsides conditions may return nearly to normal, and remain so until cold weather, overwork, automobiling, or some such factor again engorges the gland and causes it to obstruct.

If the amount of residual urine is habitually enough to cause back pressure upon the kidneys, the welfare of the patient demands its removal. If the bladder is infected, catheterization and lavage will improve rather than augment the irritation. If it is uninfected, catheterization should be undertaken only after careful consideration of the circumstances surrounding that particular case, and after a probable course of action has been mapped out. Catheterization, no matter how carefully done, may set up an acute infection. One should decide whether, assuming an important amount of residuum exists, the situation should be met by regular catheterization or by operation. My own feeling would be that more than 6 ounces of residual urine, in an uninfected bladder, demands one or the other of these measures. If infection already exists, half that amount of residuum is an indication for interference.

Shall this interference be operative, or shall it be by catheter? Upon this point it is well to take into consideration the general condition of the patient and his economic as well as his physical circumstances. A retired business man, fretting because he has nothing to do, will take vastly more interest in seeing that he catheterizes himself regularly and properly than will a laboring man still dependent upon his 
earnings. Of course, the question in any particular case may be settled by the difficulty of catheterization. The passage of instruments may become so painful or so difficult that other ways of meeting the situation will have to be sought.

There is no valid argument against operation except such a general break-down that the end seems in view. Even then, through preliminary bladder drainage, the patient may so improve that he presents an altogether different problem. Dependence upon the catheter is a poor substitute for prostatectomy. The urinary tract will certainly become infected. At any time swelling of the prostate may require surgery for its relief. The troubles inherent to catheter life are many. It is a poor make-shift:

If a patient is to live a catheter life he should be carefully instructed in regard to all its details. Soft-rubber catheters of as large a size as will pass easily should be used, though woven catheters may be necessary. The former should be rinsed out with running water after use, and once a day enough catheters to carry the patient through the next twenty-four hours should be boiled and put aside in a clean towel. Woven catheters should be carefully washed, inside and out, and kept in a flat, covered container full of boric acid solution or in a clean towel. Before catheterizing himself the patient must wash his hands. It is desirable, though not essential, that once a day he should wash his bladder, which may be done easily with a fountain syringe, the hardrubber tip replaced by a glass nozzle. As a rule, boric acid 
( 2 per cent.) should be used, and is prepared by mixing equal parts of a saturated solution and warm water. ${ }^{1}$

There are comparatively few cases in which operation, properly done, will not offer a fair chance of recovery. Age in itself is not a contraindication. I have seen men ninety years of age go through operation without being really sick at all. Kidneys so damaged that their phthalein output is less than 5 per cent. in one hour have not prevented good recovery. Disease of the circulatory system, unless far advanced, is not a contraindication to operation, for spinal anesthesia puts practically no strain upon the heart.

Third Stage.-At any time an enlarged prostate may swell and obstruct the urinary passage. The obstruction is seldom absolutely complete; a few drams may be passed at a time, but the outflow cannot keep pace with the inflow. The bladder distends. Its walls become stretched beyond the limits of their elasticity. If this condition endures for many days, uremic poisoning will set in, manifested first by urinous breath, soft distention, anorexia, nausea and vomiting, later by coma.

When a patient is first seen with retention, one must decide whether operation is to be done at once or whether catheterization should be tried.

My own feeling is that immediate operation, by which I

1 The saturated solution is made by placing from 4 to 8 ounces of boric acid crystals in a 2-quart glass bottle. The bottle is then filled with boiled water. When this stock gets low, more water is added. So long as there are crystals at the bottom of the bottle, the solution is saturated (4 per cent.). 
mean preliminary drainage of the bladkler under local anesthesia, and not prostatectomy, should be done in practically. every case. In the first place, once a patient has acute retention due to the prostate, he will never be able to empty his bladder again. If the patient's situation necessitates a delay of several hours before he can be got io a hospital, catheterization or suprapubic tapping of the bladder should be done.

I know of no operation in which the patient needs so much the daily attention of his surgeon as in prostatectomy. For this reason prostatectomy should almost never be done "out of town." The actual operation is less important than constant supervision during the postoperative period by a man trained in the management of such cases.

In regard to the immediate treatment of acute rotention there is considerable difference of opinion. All genito-urinary surgeons agree that immediate prostatectomy must not be done. To remove the prostate in the face of a distended bladder is little short of murder. Preliminary drainage is essential. Some prefer to carry out this drainage by use of the indwelling catheter. Others believe that free suprapubic drainage gives better results. Personally, I prefer the latter method. I believe the drainage is better, infection of the prostate is reduced rather than augmented, and the suprapubic wound is given a chance to become thoroughly walled off before the removal of the prostate. The chances of having the drainage get out of order, with resulting back-pressurc, are infinitely less. 
I38 AN OUTLINE OF GENITO-URINARY SURGERY

Cystotomy.-The operation of cystotomy ${ }^{1}$ is preceded by infiltration with $\frac{1}{2}$ per cent. novocain of the skin and subcutaneous tissues of the suprapubic region. An area 3 inches wide, extending from pubes almost to umbilicus, is thoroughly

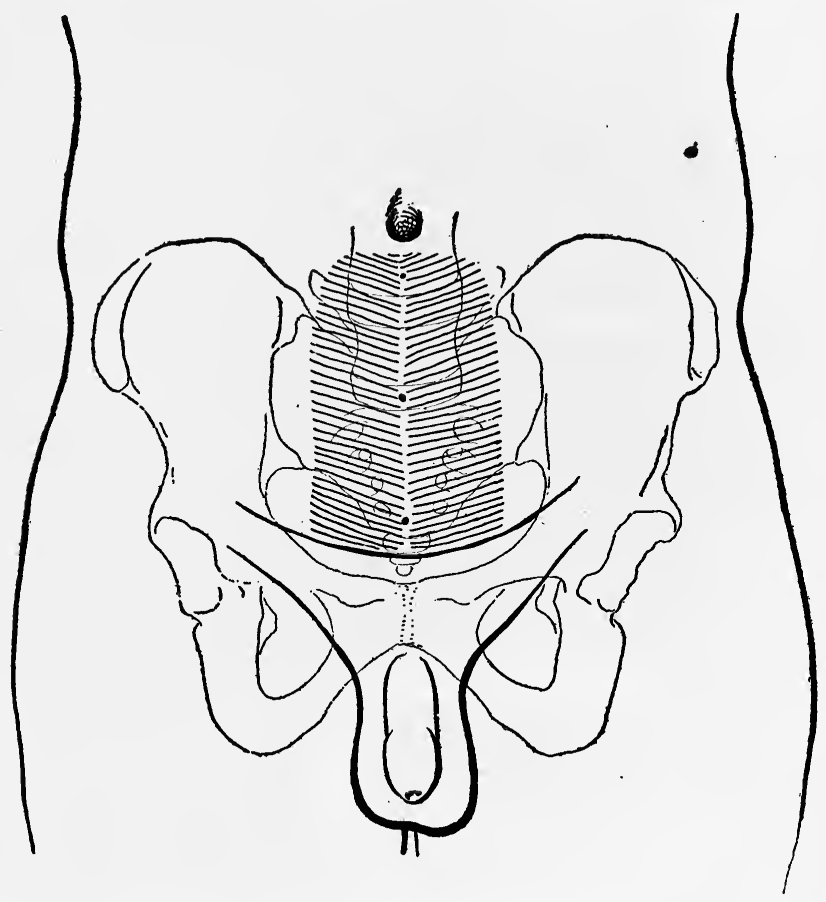

Fig. 25.-Area to be infiltrated in suprapubic cystotomy under local anesthesia.

infiltrated. The point of the needle is then pressed inward until it is felt to pass through the aponeurosis of the rectus

${ }^{1}$ I am indebted to Dr. Arthur L. Chute for this technic. I have found it always satisfactory. 
muscle. More novocain is injected under the aponeurosis and into the prevesical space. One should wait ten minutes before starting the operation. A median incision about 3 inches long is then made, the lower end being $\mathrm{I}$ inch above the pubic bone. The aponeurosis is divided and the recti

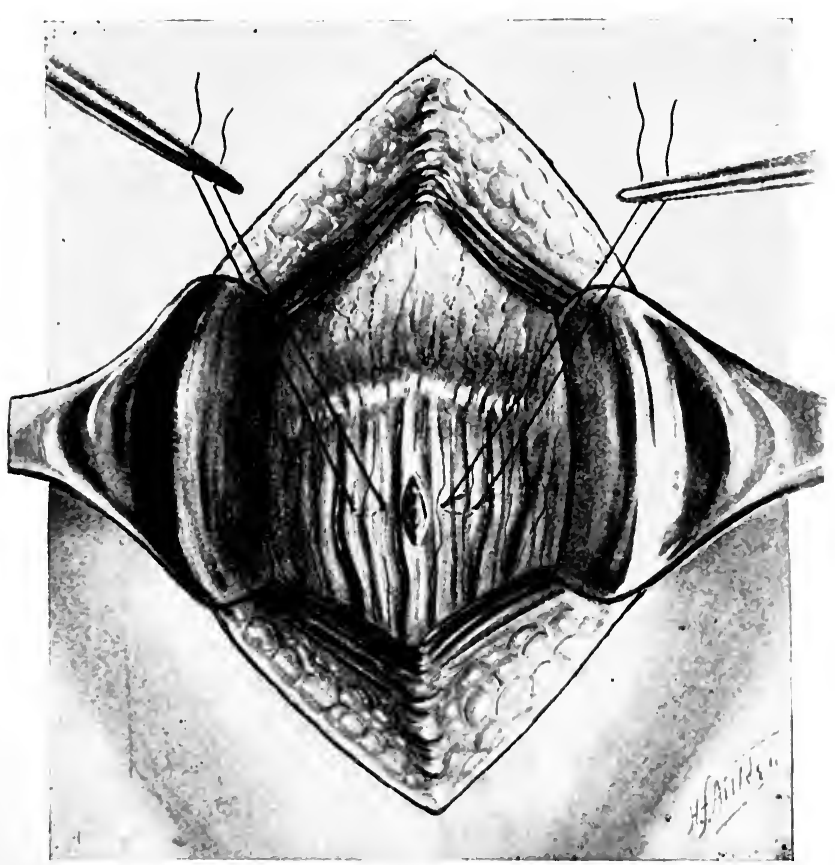

Fig. 26. - Suprapubic cystotomy. Peritoneum st ripped upward to expose bladder. Stay sutures placed and bladder wall incised between them.

separated. The peritoneum is stripped up from the front of the bladder, and that viscus is opened enough to admit the finger. Digital exploration will show the presence of tumor, stone, or diverticulum, and will give definite information as 
to the condition of the prostate. A rubber tube $\frac{1}{2}$ inch in diameter is inserted, the edges of the bladder incision are drawn against the abdominal wall by two sutures fastened

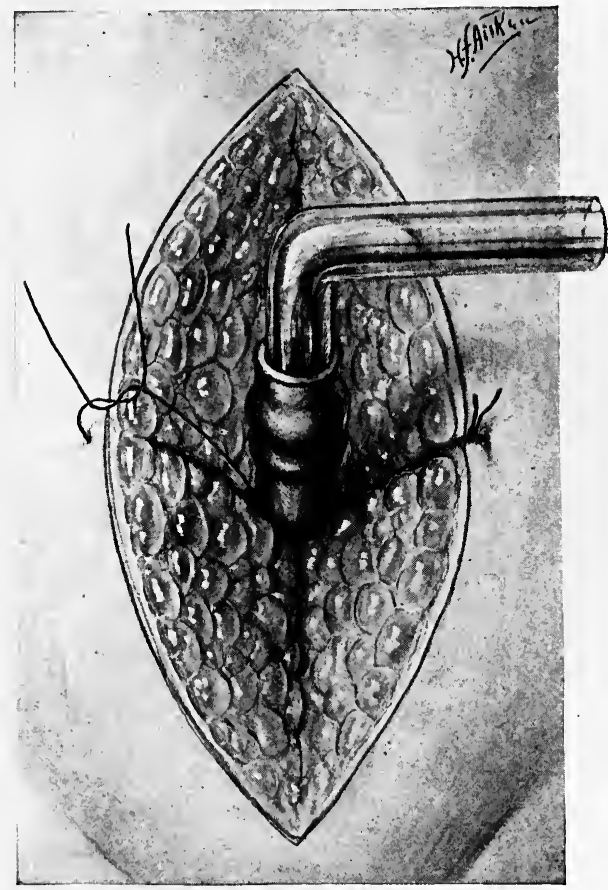

Fig. 27.--Suprapubic cystotomy. Rubber tube has been inserted into bladder and rectus fascia sutured. Stay sutures now put through skin on either side and tied loosely, so that opening into bladder can be easily found if necessary.

loosely to the fascia or skin, rubber tissue wicks are placed to the prevesical space, and the incision closed about the tube. A right-angled glass tube is placed in the bladder tube and the urine drained into a bottle. This drainage apparatus re- 
quires very little attention, and the patient can be kept perfectly dry. The drainage can be continued as long as necessary. Sometimes three months may elapse before a patient is in proper condition for the removal of the prostate. During this period the one most essential measure is the forc-

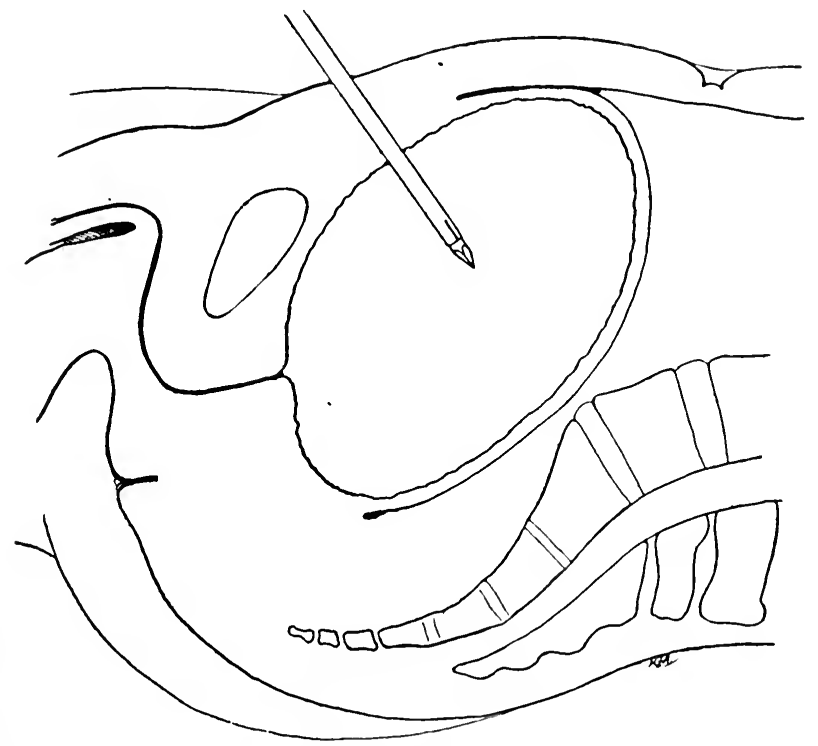

Fig. 28.-Paracentesis of overdistended bladder. Note how peritoneum is carried upward by bladder, and note also the direction in which the trocar is inserted.

ing of fluids. The twenty-four hours' output of urine should never fall below roo ounces.

A modified form of suprapubic cystotomy, which may be useful in case the above operation cannot be carried out and catheterization is deemed unwise, consists in puncturing the 
bladder above the pubes with a trocar the diameter of which is about the same as that of a 24 French sound, and through the trocar inserting a Pezzer or mushroom catheter into the bladder. This can be done without fear of entering the peritoneum only in case the bladder is greatly distended. Such bladders carry the vesical fold of peritoneum up with them, and the bladder wall just above the pubes lies directly beneath the recti muscles. The area involved should be infiltrated with novocain, the skin punctured with a knife, and the trocar inserted about an inch above the pubes, with its tip pointed toward the bifurcation of the aorta. If it is inserted perpendicularly to the table upon which the patient is lying, the hole in the bladder will be made too near the urethral orifice, and will be carried down still farther as the bladder contracts down after it is emptied.

If these operations cannot be done because of lack of operative facilities, catheterization may be tried. Select the catheter which seems most likely to enter. Don't lead up to it gradually by trying the less likely ones first. A woven catheter with bougie tip bent at an angle is an excellent instrument. So are the coudé and bi-coudé catheters, also woven. At times nothing succeeds like an old-fashioned metal catheter with prostatic curve, but the objection to it is that it is not a good instrument to leave in the urethra (Fig. 29, 3). A soft-rubber catheter upon a stylet bent into a "prostatic curve" is very useful, especially if the catheter is to be fastened in the urethra.

Very gently the catheter, well lubricated, is passed into the 
prostatic urethra. If gentle pressure does not cause it to enter the bladder, its point may be twisted from side to side in an attempt to find the passage. Force must not be used. If catheter No. I will not pass easily, try a different type. If the bleeding is profuse, give up the attempt. Do not exhaust the patient by your endeavors. It is far better to tap the bladder above the pubes than to tear the prostate and set up)
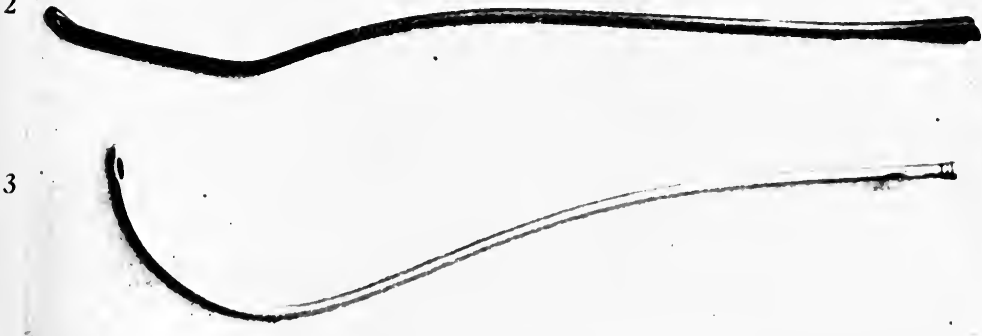

Fig. 29.-Prostatic catheters. 1. Bougie tip coulce. 2. Bi-coulé. 3. Silver catheter with prostatic curve.

an acute infection. In tapping, be careful to enter just above the pubic bone and point the needle toward the bifurcation of the aorta. If the catheter does enter the bladker, do not let your sense of satisfaction, which, egged on by the relief of the patient and the admiration of the family, may be considerable, lead you to empty the entire bladderful of urine. No more than 20 ounces should be drawn off at first; the catheter 


\section{I44 AN OUTLINE OF GENITO-URINARY SURGERY}

should then be fastened in with strips of adhesive plaster and snapped or tied off. Every hour ro ounces more may be withdrawn. After twenty-four hours or so the bladder will be emptied and the drainage may be allowed to run constantly (Figs. 30-34).

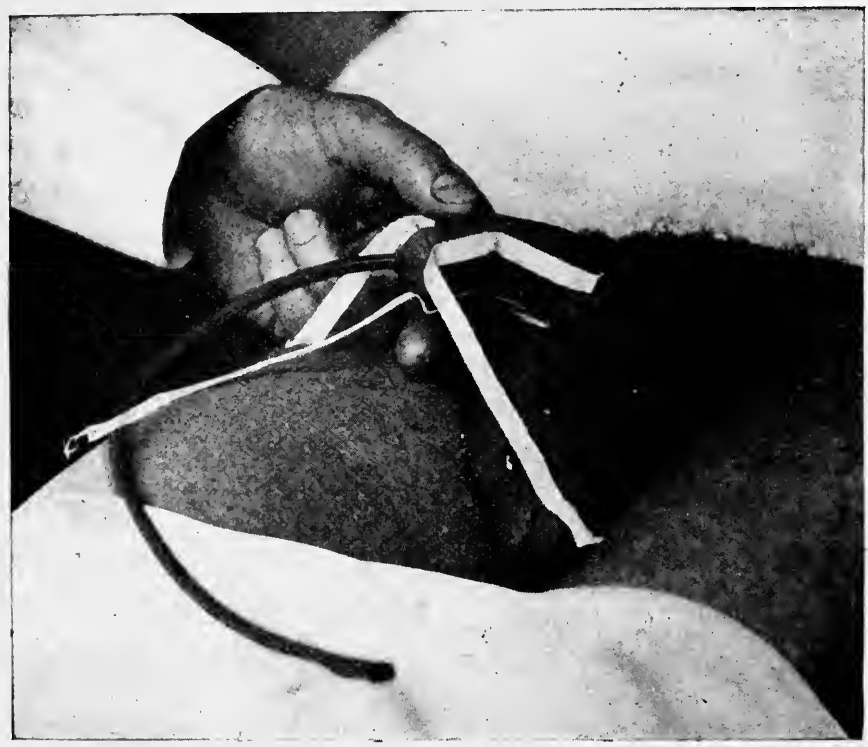

Fig. 30.-Catheter in urethra. Three longitudinal strips of adhesive plaster.

The danger from sudden emptying of an overdistended bladder is great. The wall, stretched until its elasticity is lost, collapses like a wet paper bag. Intense congestion results not only in the bladder wall but in the ureters and pelves as well. Some overdistended vein bursts, and a hemorrhage sufficient to fill the bladder with clots is the result. 
When this happens, suprapubic cystotomy must be done. When an overdistended bladder is emptied by suprapubic cystotomy these phenomena do not occur. There is no danger in suddenly emptying the bladder when it is opened. If the bladder drains well, the catheter may be left in for from three days to a week. 'The patient is given hexamethyl-

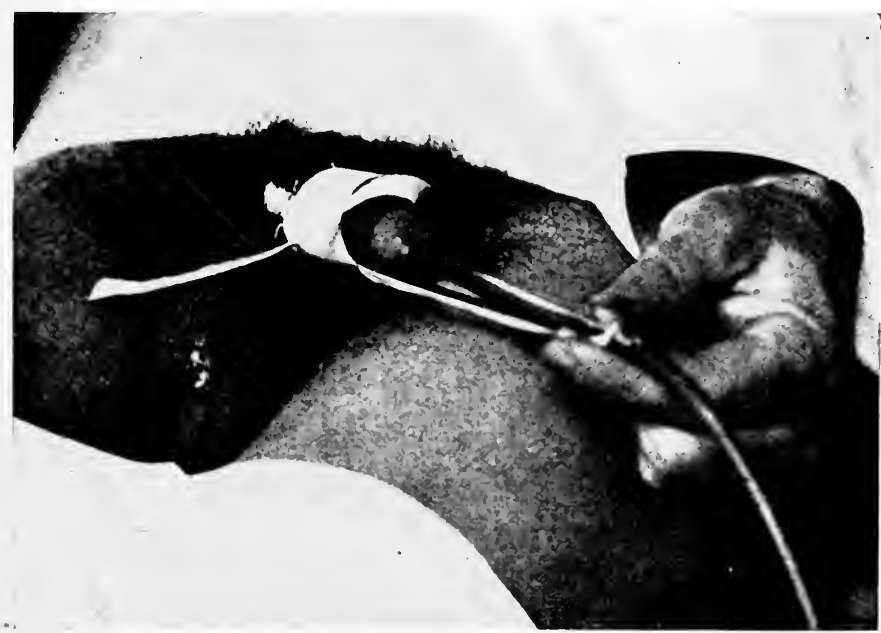

Fig. 31. - The three longitudinal strips fastened to penis by circular band of adhesive plaster. Catheter adjusted. Two of the strips being stuck on to catheter.

enamin, gr. xv t. i. d., forced fluids, and is encouraged to sit up in a reclining chair. When the urinary output has fallen from the large amount following retention to a more or less steady level, and the patient shows no evidence of uremia or septic absorption, the catheter may be removed and intermittent catheterization substituted. The patient may be 
able to void spontaneously, but only a small proportion of his urine will be thus passed. He will have to depend upon the catheter (unless he submits to operation) for the rest of his life.

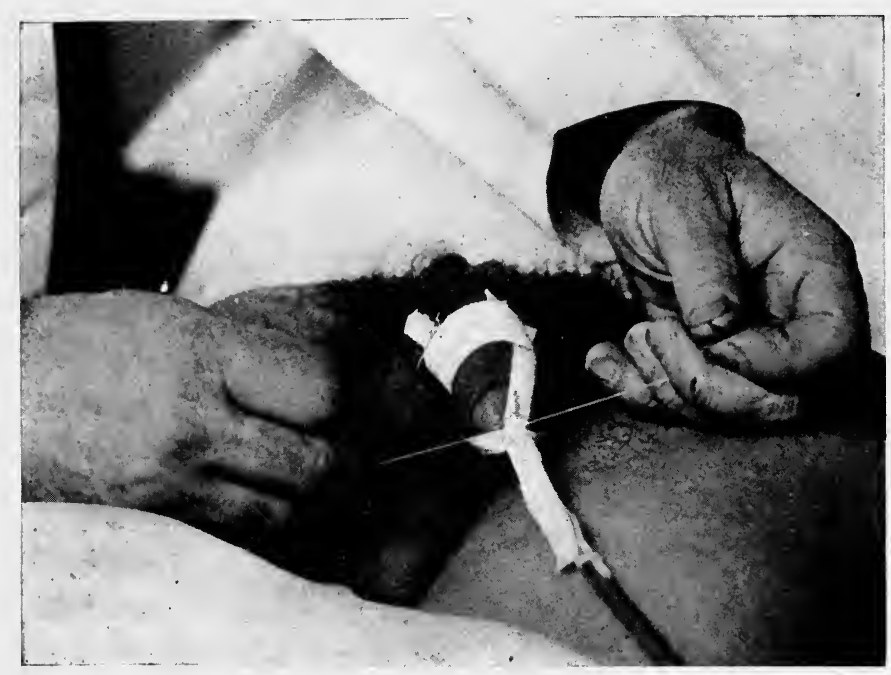

Fig. 32.-Strips of adhesive plaster, after adjustment of catheter, are fastened securely in place by a circular ligature of silk or strong thread tied close to meatus.

Prostatectomy.-As a result of preliminary drainage, whether by suprapubic tube or by urethral catheter, the kidneys gradually recover from the effects of back-pressure until they reach a fairly stable condition. The time required for this will vary from ten days to three months. In most instances from two to three weeks will suffice. The condition then attained may be far below normal, but if the kidneys are able to carry off waste products to such a degree that the 
patient's appetite and strength improve they will carry him through prostatectomy provided they are subjected to no additional strain. For this reason the choice of anesthetic is all important. Ether is distinctly bad. Gas-oxygen spares the kidneys and is, by some surgeons, considered the best anesthetic. Spinal anesthesia seems to me by all means the

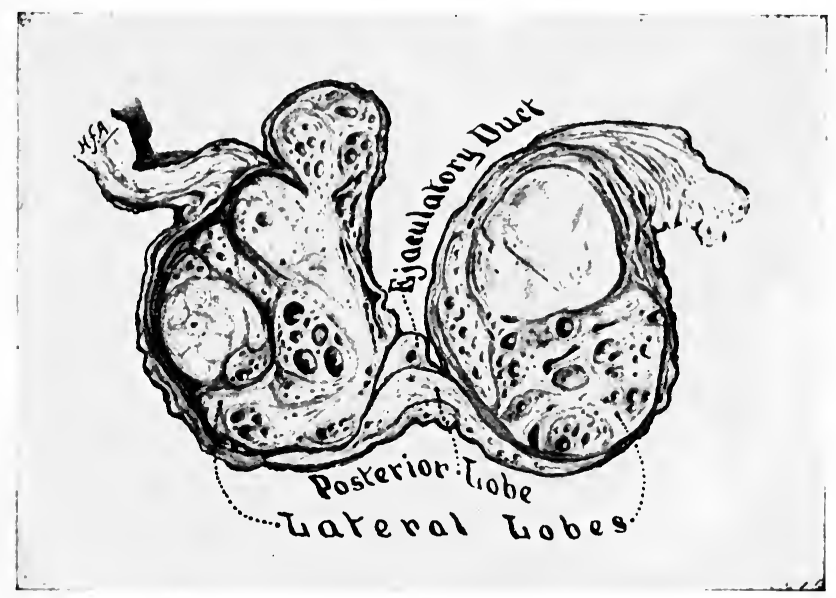

Fig. 33.-Cross-section of adenomatous prostate at level of verumontanum. Note how well defined is the line of cleavage between the lateral lobes and the posterior lobe.

safest method. If given by one familiar with the technic, it is, in my belief, devoid of danger. It places no strain upon the kidneys, and affects the heart only through the fall in blood-pressure which it not infrequently causes. Ten cg. of novocain and adrenalin dissolved in 2 c.c. of water and diluted with an equal part of spinal fluid are injected very slowly between the second and third or third and fourth lumbar ver- 
I48 AN OUTLINE OF GENITO-URINARY SURGERY

tebræ. The anesthesia lasts from an hour and a half to two hours. Within the past two years I have not seen it fail to produce anesthesia one single time. In very nervous people a few whiffs of ether may be needed for the psychic effect.

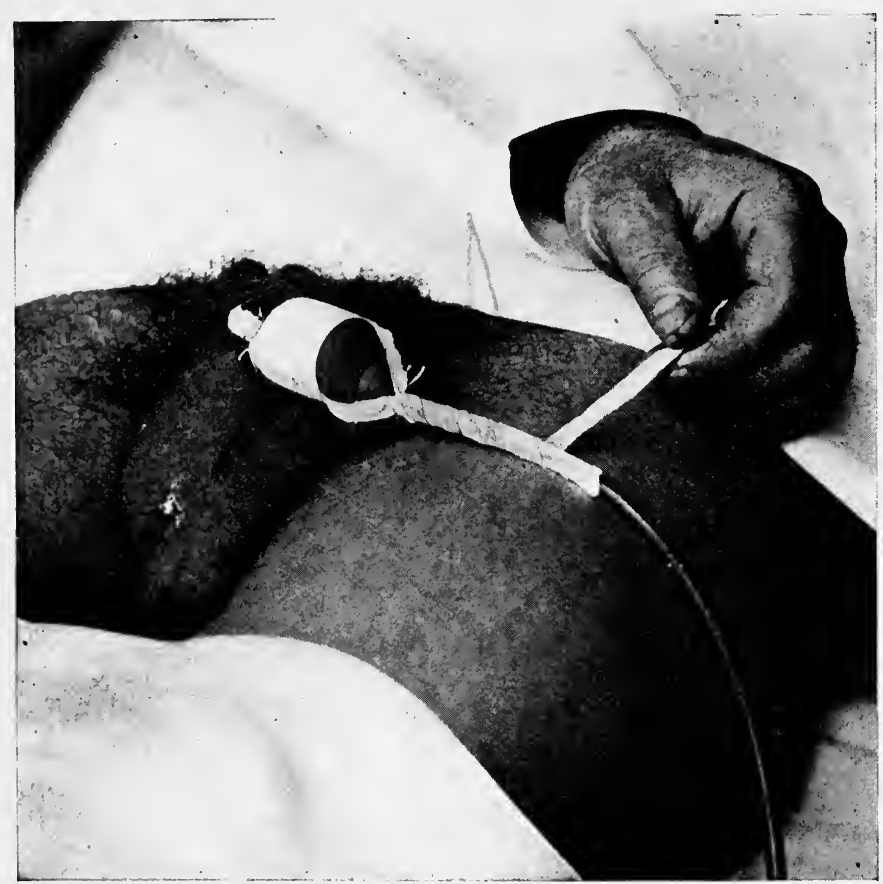

Fig. 34.-For additional security longitudinal strips are bound to catheter by spiral strip.

Only enough is given to draw the patient's attention away from the operation.

If preliminary drainage has been by urethral catheter, the bladder must be opened. A median suprapubic incision 
long enough to admit two fingers into the bladder is made. If suprapubic cystotomy has already been done, the fistula must be dilated to admit the forefinger. Enucleation of the adenomatous masses from the prostatic capsule by the suprapubic route is done by inserting the finger into the prostatic urethra, hooking it around the anterior end of one lobe, and cracking the mucous membrane along the line where this lobe meets the lateral urethral wall. The line of cleavage thus opened up is followed by the finger, and the adenomatous lobe is turned out of its bed. The other lobe is removed in the same way, and smaller adenomata beneath the bladder neck are likewise enucleated. When all masses are removed, the anterior half of the floor of the posterior urethra, bearing the verumontanum and ejaculatory ducts, is found to be intact. Behind this the urethra has been stripped of its mucosa, and consists of a raw, oozing cavity. Posteriorly this cavity is bounded by the internal vesical sphincter, which can be clearly felt.

The hemorrhage from this denuded area may be considerable. The ooze is easily controlled; the only important bleeding comes from torn vessels at the bladder outlet. To control this bleeding various methods have been used. At the Mayo Clinic, Judd sutures the torn edge of the prostatic capsule. Hagner, of Washington, has suggested the use of an inflated rubber bag to press upon the bleeding area. The Hagner bag is a pear-shaped one of soft rubber, with a tube attached to the small end. A specially devised sound is passed through the urethra from without; over the tip of this 
the tube is placed. As the sound is withdrawn it draws the tube out through the urethra. When the bag has been drawn into the prostatic cavity it is inflated, the tube is clamped and held taut. After a few hours the bag is deflated and one or two days later is withdrawn through the suprapubic wound. A catheter is sewed to the tip of the urethral tube and follows

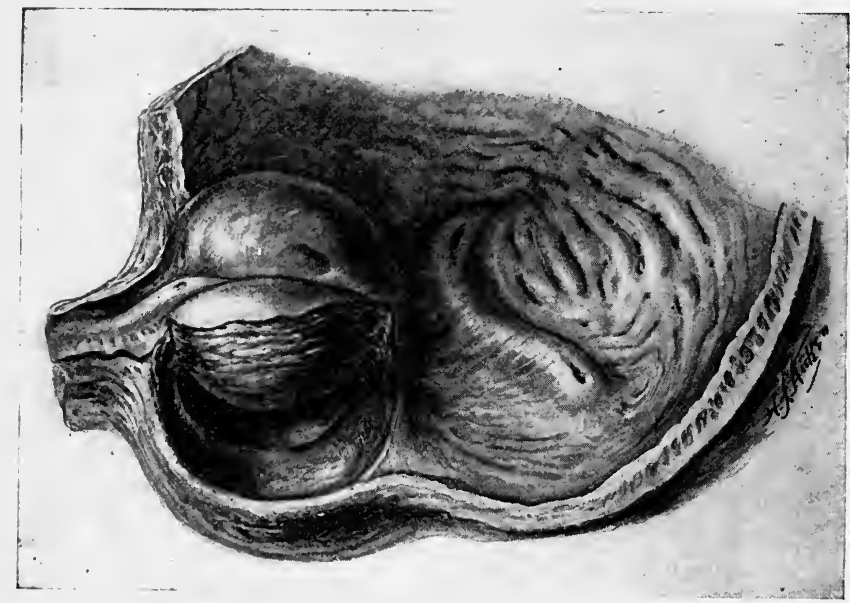

Fig. 35.-Drawing of bladder removed at autopsy, showing adenomatous prostate and the line of cleavage which the finger follows in suprapubic prostatectomy.

the tube into the bladder. This ingenious technic works very satisfactorily and does check hemorrhage, provided the tube does not break.

If the bladder has been drained by a suprapubic tube for several weeks the congestion of the prostate is usually so decreased that not much bleeding follows prostatectomy. In the two-stage operation with which I am familiar the pros- 
tate is enucleated through the small hole from which the drainage-tube has been removed. Only one finger can be inserted; in order to make enucleation possible the prostate must be supported by the forefinger of the other hand in the rectum. Enucleation through so small an aperture is often difficult, but is compensated by the absence of postoperative suppuration and by the rapid healing of the already walledoff tissues. After the enucleation a catheter is passed through the urethra and held in place by a silk suture passed through its tip and fastened to the edge of the skin incision. If there is any bleeding worth mentioning a sponge is packed into the posterior urethra about the catheter.

This second part of the two-stage method of prostatectomy, done under spinal anesthesia, really affects the patient very little. There is no cutting, and, except for the denuded urethra, no freshly opened surface to admit infection. The - kidneys are not at all affected by it, for the conditions of bladder drainage remain the same.

Prostatic Enlargement-Complete Retention, Overflow Bladder.C. G., a metal worker of sixty-nine, entered the Deaconess Hospital on September 17, 1917, suffering from acute retention. For nine years he had had more or less difficulty in roiding. He had been through two attacks of swelling of the feet, but otherwise had always been well. For the past week he had been passing very small amounts of urine frequently. He was nauseated; penis, scrotum, and legs were full of edema. By rectum, prostate was very large and elastic. Stricture was ruled out by the passage of a catheter through the cut-off muscle. Reflexes were normal. Nothing abnormal was found in cardiac condition. Face drawn and pale, tongue thickly coated. Under local anesthesia the bladder was opened suprapubically, with escape of a very large amount of concentrated, foul urine, the last of which contained thick pus. A large rubber tube was inserted into the bladder and the wound closed about the tube. 


\section{I52 AN OUTLINE OF GENITO-URINARY SURGERY}

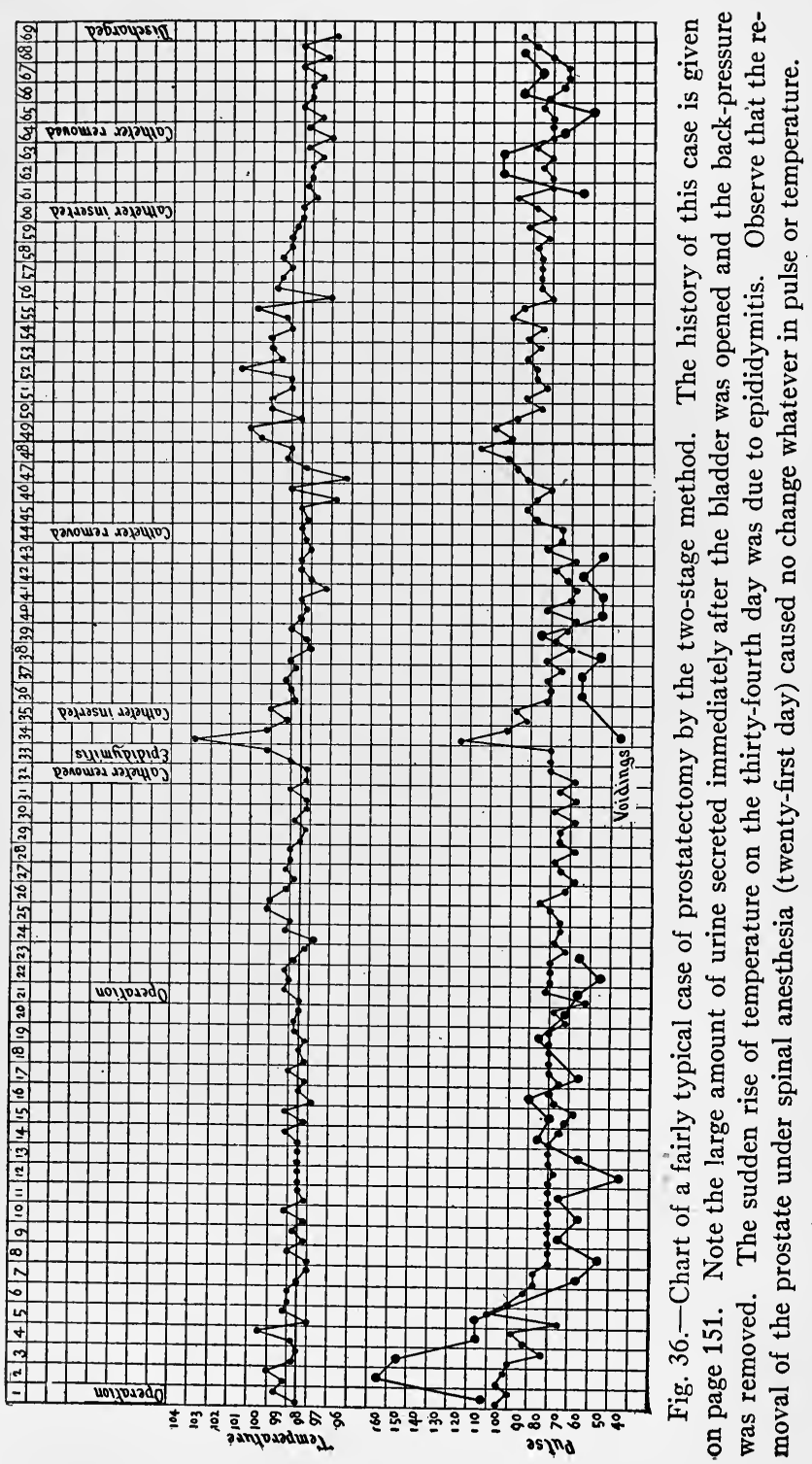


Fluids were forced. The patient reacted very well. His edema disappeared within forty-cight hours, appetite returned, and in three weeks he was in condition for prostatectomy. Under spinal anesthesia large masses of adenomatous tissue were enucleated through the suprapubic fistula. A catheter was passed through the urethra and fastened to the edge of the suprapubic wound with a silk suture. There was slight oozing, and a sponge was packed into the prostatic cavity. This was removed two days later. In the second week epididymitis developed, but quieted down with support and hot flaxseed poultices. The catheter was removed 23 days after operation. The wound broke open, however,

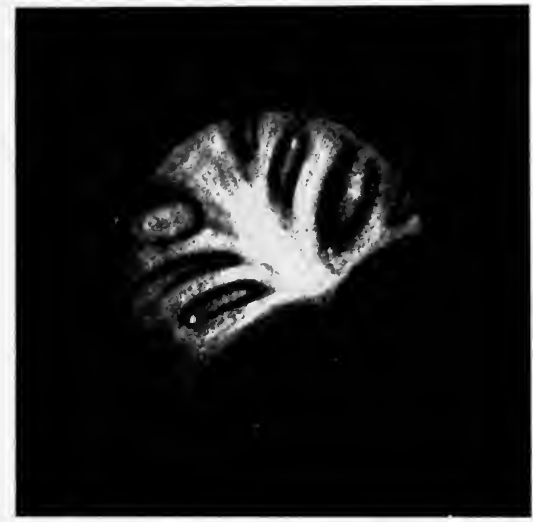

Fig. 37.-Cystoscopic picture in a case of enlarged prostate. The dark area is a portion of the prostate projecting into the bladder. In the background is seen the trabeculated bladder wall.

necessitating the replacing of the catheter. After the wound had firmly healed it was found that there was a residuum of 16 ounces. Cystoscopy showed no cause for this except a flabby bladder. A 32 French sound passed easily. The bladder was irrigated daily with potassium permanganate solution, and when the patient left the hospital on November 29, 1917, after a stay of ten weeks, he had only 3 ounces residuum. He came to my office every week for irrigation. The residuum decreased somewhat, so that when last seen he had between 1 and 2 ounces only, and felt perfectly well. He got up only once at night, his feet no longer swelled, and his general condition was excellent. 
The perineal route is favored by some operators, who use it altogether. Unquestionably it is less shocking to the patient. The wound is smaller. The chief drawback is, in my experience, the tightness of the bladder neck which occasionally follows. A diaphragm is sometimes left at the internal orifice, which prevents a perfect functional result. There is some danger of interfering with the control of urination, and a bare possibility of getting a recto-urethral fistula.

For certain types of cases it is the operation of choice. Men with fat abdomens, in whom, in order to permit suprapubic enucleation, a large wound would have to be made, are best done by the perineal route. The small fibrous prostate, which cannot be enucleated by the finger, can be dissected out through a perineal incision and the bladder neck widely opened.

The operation is best done through a curved pre-anal incision. The central tendon of the perineum is cut, allowing the rectum to fall back. The rectal aspect of the prostate is exposed, an incision made into the urethra at the apex of the prostate, and the gland drawn down by Young's tractor. The lobes may be enucleated through incisions made through the posterior capsule or may be enucleated through the urethra. A double-barreled tube drains the bladder through the perineal wound.

J. P., age sixty-nine, a real estate dealer, was seen by me November 9,1917 , in a condition of almost total retention. He was able to void small quantities of bloody urine. The bladder was distended almost to the umbilicus. Prostate, by rectum, was very large' and elastic. For some months he had had nocturia and frequent burning urination, 
One week ago his bladder had been washed out and 1 pint of residual urine withdrawn. Since that time he had been feeling miserable. He was a very obese man. Heart showed a systolic murmur, but there was no evidence of decompensation. The reflexes were normal. November 10th, under local anesthesia, median suprapubic incision. A thickened bladder was opened, and, upon exploration with the finger, it was found that the incision had been made into a diverticulum of about 2 ounces capacity which was situated on top of the bladder. The urine was very foul. The diverticulum was dissected out and the stoma resected. A stone the size of a plum was removed from the bladder. A suprapubic tube was sutured in the bladder.

The wound, infected by the very dirty urine, broke down, but aside from that convalescence was normal. By December 19th the suprapubic wound had shrunken down about the tube and was well healed. The urine had cleared up and the patient was in good condition.

December 19th, spinal anesthesia. It seemed clear that owing to the patient's obesity suprapubic enucleation would be very difficult. Perineal prostatectomy was therefore done, and two adenomatous lobes, each the size of a horse-chestnut, were enucleated. There were many small concretions of brownish color between the lobes and the capsule of the prostate. Convalescence from this operation was very good, except for a short attack of epididymitis, and the patient left the hospital on January 6th with less than 1 ounce residuum.

Postoperative Treatment.-After any operation upon the prostate the most important thing is to keep up the output of urine. Unless large quantities of urine ( 100 ounces or more every twenty-four hours) are excreted the kidneys may fail to perform their full duty. Uremia sets in, evidenced by dry tongue, soft distention, anorexia. If these signs appear, the patient should be given salt solution under the breast, 750 c.c. or more at a time. Besides the effect of the fluid upon his kidneys, he is tremendously impressed with the unpleasantness of the procedure, and will suddenly realize the necessity of drinking water. A patient of mine had subpectoral salt solution once or twice a day for forty consecutive days before 


\section{I56 AN OUTLINE OF GENITO-URINARY SURGERY}

he regained his appetite and shook off the signs of impending uremia.

Second only in importance to plenty of fluid is the matter of exercise. Shaky old men must be got out of bed earlyas soon as the urine ceases to be bloody-and must be made to walk and go into the fresh air. Only by closest observance of these principles can some of these old men, whose kidneys are all but gone, be dragged back to health. Once they begin to recover, the improvement in general condition is nothing short of marvelous. The game is worth the candle, but only by the most assiduous care can it be won.

After-treatment of Prostatectomy.-The patient discharged by the surgeon after a successful prostatectomy is seldom cured of his cystitis. If he is left untreated after his discharge from the hospital he runs an excellent chance of having an exacerbation of his bladder infection. This causes swelling of the structures about the vesical orifice, with the retention of a small amount of urine. This residual urine does not increase to more than 3 or 4 ounces, but is sufficient to aggravate the cystitis and to assist greatly in the formation of bladder stone. Every case after prostatectomy should be seen, during the first three months, at least once every two weeks. The condition of the urine and the amount of residuum should be followed. If there is residual urine or if cystitis exists, the bladder should be washed frequently, and the patient should take either sandalwood oil, ro minims t. i. d., or hexamethylenamin, 45 grains a day, with sodium acid phosphate, ro grains t. i. d., if the urine is not already acid. 
Dilatation of the bladder neck with sounds improves the action of the sphincter and diminishes residuum. Even after the urine becomes free from pus the patient should be seen occasionally, as his bladder may be reinfected from the chronic pyelitis which is likely to have originated during his preoperative days.

The Small Fibrous Prostate.-There is a type of obstruction at the bladder neck caused by contraction of the internal urethral orifice. This contraction is due to the presence of connective tissue which has formed beneath the mucosa as a result of a previous inflammatory process. The outlet of the bladder is puckered into a tight orifice. The effect of this upon urination may be heightened by the deposit of more fibrous tissue throughout the prostate itself. The condition is called by various names-median bar obstruction, small, fibrous prostate, contracture, prostatism sans prostate. It is met in men of thirty-five years of age and upward, and may be responsible for retention of urine in amounts varying from $\mathrm{I}$ or 2 ounces up to 90 or more. Usually the vesical orifice grips a sound, but in many cases it will admit a soft-rubber catheter easily.

The diagnosis depends upon the exclusion of other causes for retention, namely, diverticulum, nerve lesions, and cancer. Adenomatous enlargement of the prostate is ruled out by rectal and cystoscopic examination.

The treatment consists of removal of the obstruction either by the use of Young's punch or by perineal prostatotomy. The punch operation is done through the urethra 


\section{I58 AN OUTLINE OF GENITO-URINARY SURGERY}

and may require only local anesthesia. Improvement is apt to be only temporary. Prostatotomy-the excision of a segment of the contraction ring-aided by the removal of as much prostatic tissue as possible, is the most satisfactory measure. It is done in the same way as a perineal prostatectomy, except that the obstruction is cut out instead of being enucleated with the finger.

Cancer of the Prostate.-To every 5 or 6 cases of benign enlargement of the prostate there is I case of cancer. In the great majority of cases cancer of the prostate, as has been shown by Geraghty, originates in the posterior lobe. This is the area beneath the verumontanum, and is distinct from the so-called "middle" lobe and lateral lobes in which adenomatous changes occur. The growth extends upward along the seminal vesicles, and although it may appear in the mucous membrane of the posterior urethra and trigone, it is not likely to cause retention of urine until the process is well advanced. The symptoms are due to (I) involvement of sensory nerves of the posterior urethra, causing frequency; (2) ulceration of mucosa, causing hematuria; (3) pains in back and legs, due either to involvement of nerves in the growth or to metastases in the spine. Later in the course of the disease difficulty with urination is met, and a fact peculiar to cancer of the prostate is the unusual difficulty of catheterization and the undue amount of pain and bleeding caused by it, compared to the relatively slight obstruction to the outflow of urine.

Upon rectal examination the finger strikes a hard area in 
the anterior rectal wall just inside the internal sphincter. This area may be knobby and plastered on to the pelvic wall, forming a solid platform across the pelvis. It may be felt extending upward along the vesicles. It feels altogether different from the smooth, elastic, well-defined adenomatous prostate.

In comparatively few cases adenomatous prostates undergo malignant degeneration. In that event, diagnosis is more difficult, and is based upon the presence in the adenomatous lobes of areas of stony hardness. As inflammation may cause similar areas, it is readily seen that the carly diagnosis of such a case is a matter of considerable uncertainty. Cystoscopy may show a characteristic knobbing of the vesical surface of the prostate, provided the growth is in that direction.

Prognosis.-Cancer of the prostate is of slow growth. Men who, when first seen, have already well-marked incluration of the prostate and vesicles, may live for several years more. Very probably their cancer had begun several years previously, thus covering a period of from five to eight years in all. I have often been surprised at the extent of the growth in men who have just begun to have symptoms. Sometimes general carcinomatosis will claim its victim before the bladder is affected.

Treatment.-An attempt at radical cure of cancer of the prostate is made by Hugh Young in his operation for total removal of prostate and seminal vesicles. Aside from the technical difficulties of the operation one has to contend with the difficulty of making a definite diagnosis of cancer while it is 
yet early enough to attempt a radical cure. For one reason or another the operation has not been generally adopted.

In cases in which obstruction to the urinary outflow exists the prostate may be attacked by the perineal route and as much as possible of the malignant tissue nibbled away.

Within the past few years the use of radium in the treatment of carcinoma of the prostate has gained several enthusiastic supporters. Hugh Young applies it by rectal and urethral approaches. Barringer inserts a needle containing a capsule of radium into the prostatic tissue by way of the perineum. Both have observed some very satisfactory results, about one-third of Young's cases being definitely improved, with marked recession of the growth.

I have tried a combination of perineal operation and radium treatment. After removing as much of the malignant tissue as possible, and restoring the patency of the bladder outlet, I place tubes of radium emanations in each lobe, withdrawing them by means of strings brought out through the perineal wound. Too few cases have been treated this way to justify any opinion as to the value of the method.

By means of the above measures the patient with carcinoma of the prostate can be eased along until the ureters become obstructed by the growth, or until general carcinomatosis takes place. As a last resort suprapubic cystotomy may be necessary.

It will be five years at least before we can judge of the real value of radium in this condition. At present it is the only ray of light in an otherwise very gloomy situation. 
Cancer of the Prostate-Perineal Prostatectomy.-D. C., a gardener seventy-four years of age, was referred to me on November 6,1917 , for partial retention of urine. He had always been well until a few months ago, when he began to notice difficulty in passing water. This has increased and he has required catheterization. His doctor found a residuum of 16 ounces.

Examination showed a rather feeble old man, whose reflexes and general physical examination were negative. By rectum, the prostate was of stony hardness, not much enlarged and not adherent to the pelvic walls. A woven catheter was passed with some difficulty, and fastened in the urethra. The bladder was drained by catheter for three day's. The urine was only slightly infected (more characteristic of carcinoma of prostate than of adenomatous obstruction).

November 9th, under ether, perineal partial prostatectomy was done, as much as possible of the carcinomatous gland being nibbled away. The growth could be felt extending upward beneath the trigone. Microscopic examination showed scirrhous carcinoma. A perineal tube was left in for two days, and a catheter which had been inserted at operation was left in for about ten days. After its removal there was leakage through the wound and the catheter was replaced. I )ecember 10,1917, he left the hospital. The wound was healed, there was no residuum. April 8, 1918, he came to see me to learn if he could go to work. He looks and feels well. Color good; has evidently gained weight. He has no urinary symptoms and gets up only once at night. Urine slightly hazy. No residuum. No abdominal masses. By rectum, however, the whole base of the bladder feels indurated as high as the finger can reach.

Lowsley, O. S.: The Gross Anatomy of the Prostate Gland and Contiguous Structures, Trans. Amer. Urolog. Assoc., 1914, viii, 172-195.

Савот, H.: The Operative Treatment of Prostatic Hypertrophy, Lancet-Clinic, March 8, 1913.

Squier, J. B.: Vital Statistics of Prostatectomy, Surg., Gynec., and Obst., October, 1913, 433-436.

Wilson, L. B., and McGrati, B. F.: Surgical Pathology of the Prostate, Surg., Gynec., and Obst., December, 1911, 647-681.

Chute, A. L.: Some Things that Influence the Mortality after Prostatectomy, Boston Med. and Surg. Jour., 1914, clxxi, 808-812. The Small Fibrous Prostate, Boston Med. and Surg. Jour., 1910, clxiii, 606-610.

Chetwood, C. H.: Different Types of Fibrous Obstruction of the Bladder Outlet and their Treatment, Surg., Gynec., and Obst., 1915, $\mathrm{xxi}, 202-205$. 


\section{I62 AN OUTLINE OF GENITO-URINARY SURGERY}

Smrth, G. G.: Spinal Anesthesia in Urology, Interstate Med. Jour., 1914, xxi, No. 11. Experimental and Clinical Observations on the Blood-pressure in. Spinal Anesthesia, Boston Med. and Surg. Jour., 1915, clixxiii, 502-504.

Geraghty, J. T.: Pathology of Cancer of the Prostate, Reports of Meetings of N. E. Branch of the American Urological Association for the Winter of 1912-13.

Barringer, B. S.: Radium in Treatment of Cancer of Prostate and Bladder, Jour. Amer. Med. Assoc., 1917, lxviii, 1227.

Young, H. H.: Cancer of Prostate, Ann. Surg., 1909, 1, 1144-1233.

Young, H. H., and Frontz, W. A.: Some New Methods in the Treatment of Carcinoma of the Lower Genito-urinary Tract with Radium, Jour. Urology, 1917, i, 505-542. 


\section{CHAPTER XI}

\section{DISEASES OF SCROTUM AND TESTICLE}

Hydrocele; Spermatocele; Hematocele.-The testicle hangs in the scrotum suspended by the spermatic cord and attached to the scrotal wall by the reflection of the tunica vaginalis on to the middle third of the epididymis. Except at its attachments the testis is covered by the visceral layer of the serous membrane which lines the sac (tunica vaginalis). Lining the cavity of the scrotum is the parietal layer of this same membrane. Both are derived from the peritoneum during the descent of the testis. In the sac thus formed three kinds of fluid may accumulate. A collection of watery fluid is a hydrocele; of spermatic fluid, a spermatocele; of blood, a hematocele.

Hydrocele fluid is usually a clear, yellow, slightly viscid liquid. Its composition may be changed by the addition of products of inflammation, such as fibrin, pus-cells, and blood. Hydrocele is idiopathic-i.e., we do not know what causes it-or symptomatic; the latter is frequently present in many diseases of testicle and epididymis.

Spermatocele follows a weakening of the wall of some portion of the seminal tract, usually in the epididymis. The weakened portion of the wall distends with accumulated fluid, which is thin, grayish-white in color and contains 
spermatozoa, and forms a sac inside the cavity of the tunica vaginalis.

Hematocele follows an injury to the scrotum and consists of a collection of blood, which may be clotted in the peritesticular sac.

All of these conditions cause enlargement of the scrotum. The tumor is elastic, fluctuant, and, unless acute, is not tender. The amount of fluid may vary in quantity from a thin layer overlying the testis to an accumulation of a pint or more. In hydrocele and hematocele the testis is surrounded by fluid and may be difficult to feel; in spermatocele the testis can usually be palpated outside the sac of fluid. The ability of the fluid to transmit light when placed before a strong light and viewed through a tube, pressed against the scrotum on the opposite side from the illumination, indicates hydrocele or spermatocele. Hematocele is, of course, opaque, and often feels firm, owing to the presence of clots. The differentiation between hydrocele or spermatocele and hernia must always be carried out. The former transmit light; hernia does not. When the patient coughs, hernia gives an impulse to the finger placed within the external inguinal ring. In hydrocele, one should be able to hold the spermatic cord between the fingers and rule out absolutely the presence of an impulse on coughing. There is a venous wave caused by coughing or forced expiration which may simulate a hernial impulse.

Given a fluctuant, non-tender, or only slightly tender tumor of the scrotum, one rules out by the history traumatic 
hydrocele and hematocele. It is a noteworthy fact that many cases of scrotal disease attribute their trouble to an injury, when really the accident which they imagine caused the abnormality simply served to draw attention to the scrotum. Traumatic hydrocele and hematocele do not occur except as a result of injury of really considerable violence.

Hernia, traumatic hydrocele, and hematocele being ruled out, the diagnosis must be made between spermatocele, idiopathic hydrocele, or hydrocele accompanying some underlying disease of testis or epididymis. This is done by tapping the sac and emptying it of the fluid. The character of the fluid withdrawn will tell a good deal about its etiology. Grayish white fluid denotes spermatocele; turbid yellow fluid, symptomatic hydrocele. Clear yellow fluid may be either idiopathic or symptomatic hydrocele.

For tapping a hydrocele of small amount one may use a Io-c.c. syringe equipped with a fairly large needle (Fig. 38 ). For emptying larger collections of fluid a trocar of small caliber is better. The scrotum should be washed thoroughly with soap and water. The spot to be tapped may be rubbed with alcohol. The instruments must be boiled. The operator stands on the patient's right; with his left hand he encircles the scrotum above the tumor, and by pressure from above downward renders the skin smooth and the tumor tense. The trocar, or needle, held in the right hand with the forefinger upon the shaft $\mathrm{I} \frac{1}{2}$ inches from the point, is plunged with a quick motion into the tumor in its lower, anterior quadrant. The point must be carried forward to avoid stabbing the 


\section{I66 AN OUTLINE OF GENITO-URINARY SURGERY}

testis. The forefinger serves as a guard to prevent the instrument from entering too far; when the point is felt to be free in the sac, the obturator is removed and the fluid allowed to escape into a basin placed upon the patient's thighs.

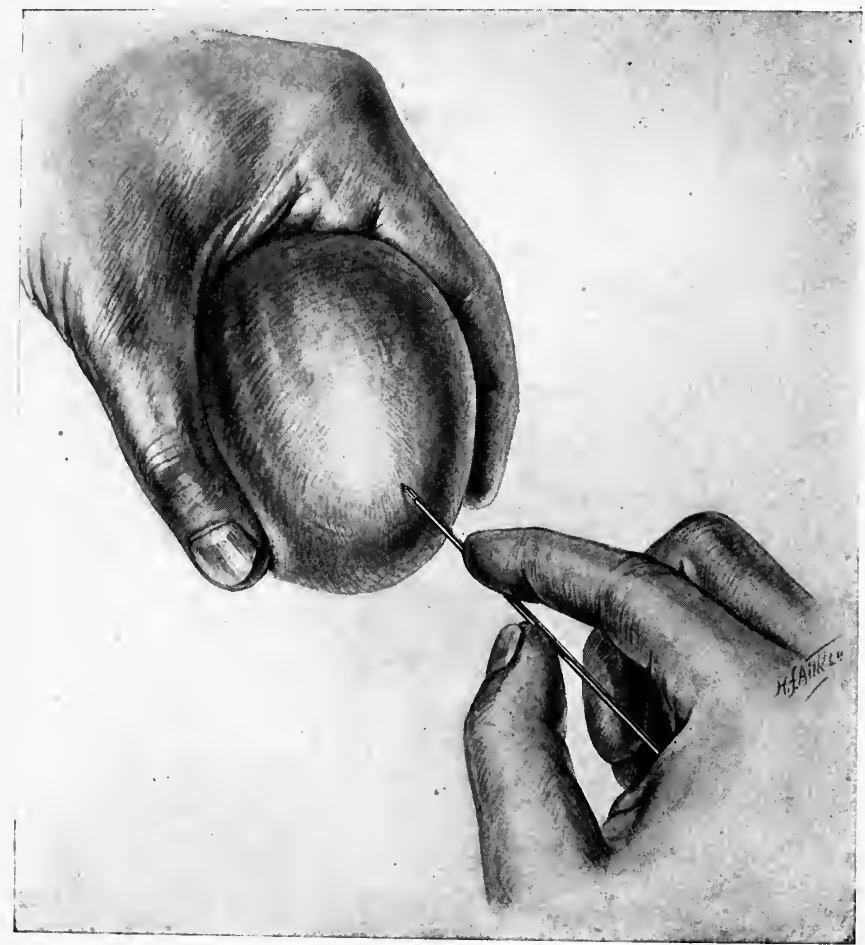

Fig. 38.-Tapping a hydrocele.

During this process pressure exerted by the left hand upon the tumor should be continued until every bit of fluid is withdrawn. Upon removing the needle the wound closes by the contraction of the dartos muscle. When the scrotum 
is emptied of fluid its solid contents become easily palpable. They should be carefully outlined; the size and consistency of the testicle noted, and the size, hardness, and sensitiveness of the epididymis. If disease is found, the cure of the

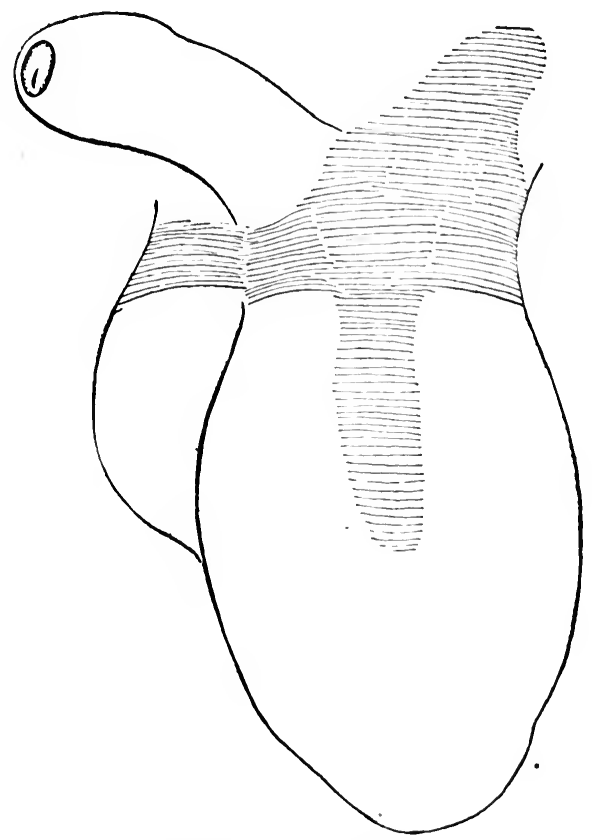

Fig. 39.-Area to be infiltrated in operations upon the scrotum under local anesthesia. The line of infiltration extends entirely around the scrotum.

hydrocele depends upon the relief of this; if the fluid be due to spermatocele or to idiopathic hydrocele, radical cure of the condition should be sought by operation. Spermatocele and hydrocele almost always refill after being tapped, and 


\section{I68 AN OUTLINE OF GENITO-URINARY SURGERY}

the removal or obliteration of the sac which secretes or contains the fluid must be carried out to effect a cure. The obliteration of the sac by the introduction of irritating material - catgut (Whitney), carbolic acid (Keyes)-has seemed to us an unnecessarily blind procedure, when radical cure by operation can be done with so little risk under local anesthesia.

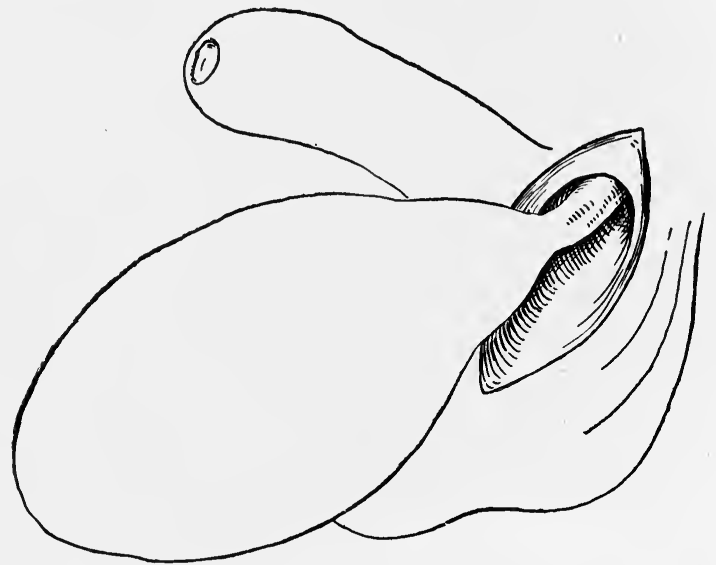

Fig. 40.-Hydrocele sac dissected free from scrotum and attached only by spermatic cord.

To secure local anesthesia for operations upon the scrotal contents the following technic has been found very satisfactory: 60 to 80 c.c. of $\frac{1}{2}$ per cent. novocain with adrenalin is used, injected with a 5- or ro-c.c. syringe equipped with a sharp needle $\mathrm{I} \frac{1}{2}$ or 2 inches in length. The proposed line of incision on the front of the scrotum is infiltrated. The infiltration is carried upward to the level of the external inguinal ring. Where the spermatic cord crosses the pubic bone it is 
picked up and held firmly between the fingers. About 5 c.c. of the solution is injected into the cord. A line of infiltration is then carried all the way around the base of the scrotum. After the injection the operator should wait ten minutes before beginning the operation. A vertical incision is

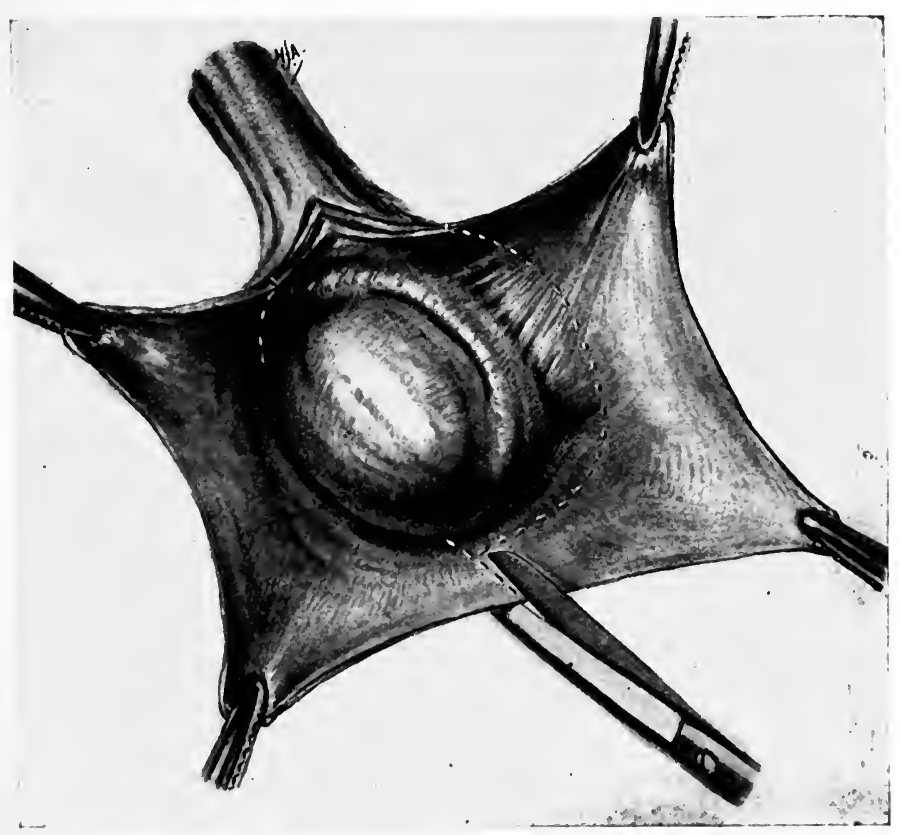

Fig. 41.-Resection of the hydrocele sac.

made on the anterior surface of the scrotum. It is carried down to the tunica vaginalis, which is not opened. By blunt dissection the tunica with its contents is freed from the scrotum. The fluid may be allowed to escape, so that the tunical sac may be extruded through the skin incision. The 
I70 AN OUTLINE OF GENITO-URINARY SURGERY

tunica is then opened and its contents inspected. If there is a hydrocele, the tunica vaginalis should be cut away on all sides $\frac{1}{2}$ inch from its attachment to the testis. If the cut

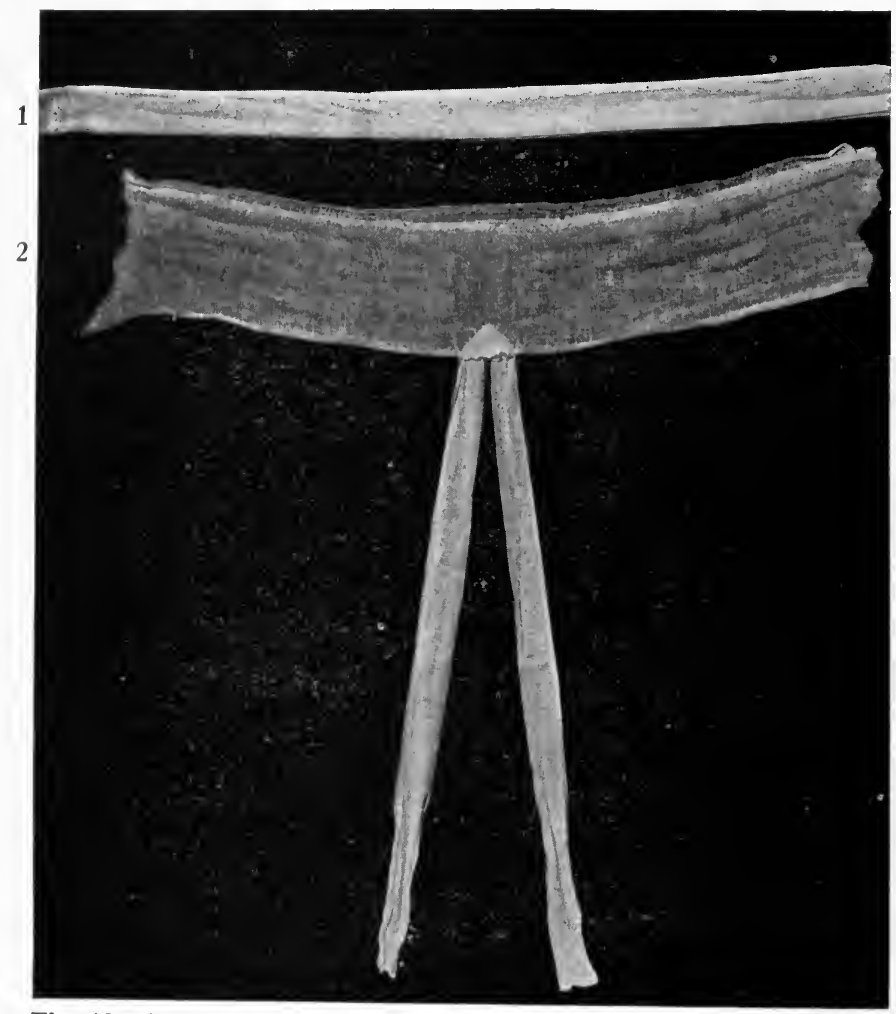

Fig. 42.-Parts of the Alexander bandage: 1. Upper, waist band. 2. Lower, scrotal bandage.

edge bleeds it should be whipped with plain catgut. Spermatocele is treated in practically the same way. The pouch which contains the spermatic fluid must be cut away as well 
as the tunica vaginalis. The testis is then replaced in the scrotum, a rubber-tissue drain inserted, and the skin incision closed with silkworm-gut sutures. In operating upon the scrotum under local anesthesia great care must be used to pick up every bleeding point. Smaller vessels, in fact, may be so contracted by the adrenalin that they do not bleed during the operation, but will ooze after the adrenalin has been absorbed.

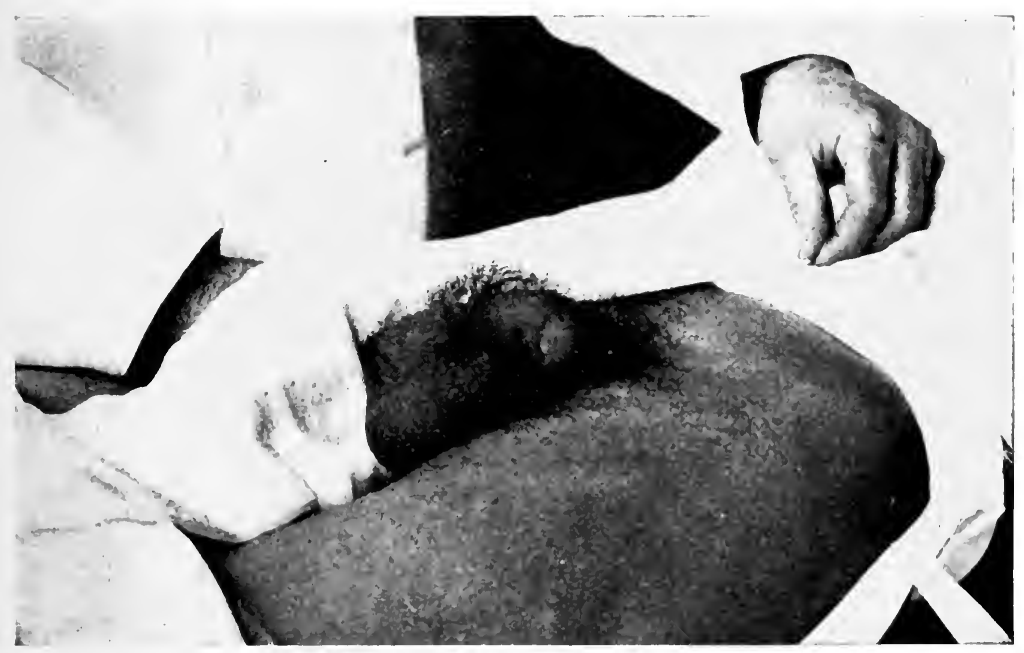

Fig. 43.-Right hand supports center of bandage in perineum.

This is especially likely to happen when considerable raw surface is exposed, as occurs in the freeing of a large hydrocele sac. Careful hemostasis and a tight dressing are, therefore, very necessary.

To secure pressure after operations upon the scrotum an Alexander bandage (so named after the late Doctor Samuel Alexander of New York) may be used (Figs. 42-47). It 
I72 AN OUTLINE OF GENITO-URINARY SURGERY

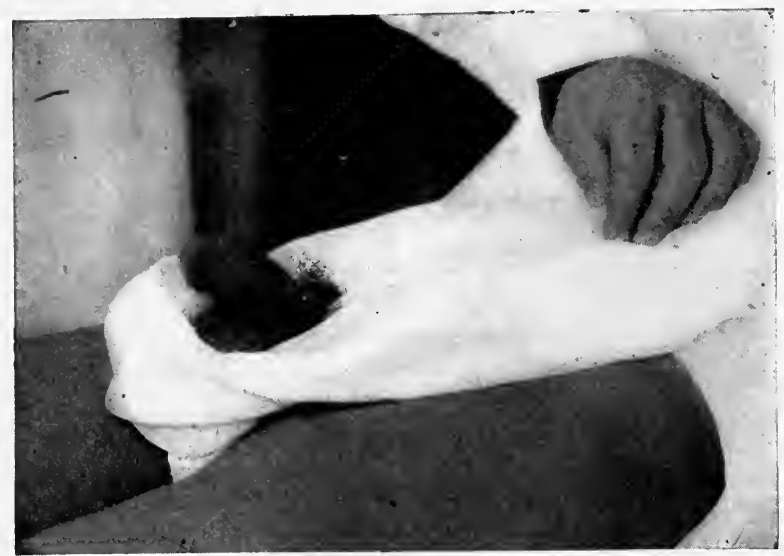

Fig. 44.--Lower edge of bandage pinned tightly about scrotum.

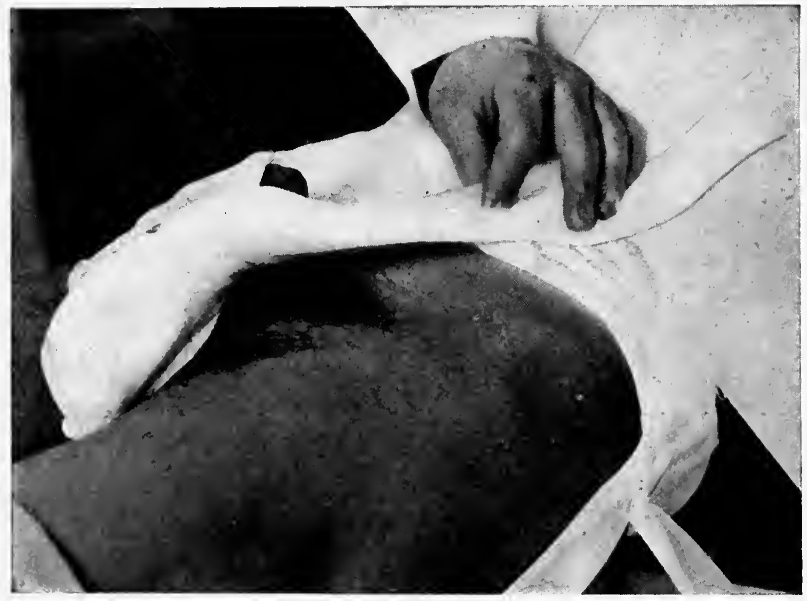

Fig. 45.-Upper edge of bandage pinned over scrotum.

gives the necessary compression and elevation, and does not interfere with manipulations about the anus. The bandage consists of a strip of gauze 3 feet long by 20 inches wide, folded 
twice so as to make a strip 3 feet long and 5 inches wide. To the middle of one edge is sewed the middle point of a piece of 2-inch bandage 3 feet long. Another strip of gauze or flannel is needed to serve as a waist band. The point where the bandage is sewed to the gauze is placed on the perineum. The bandage ends pass one under each thigh and fasten to
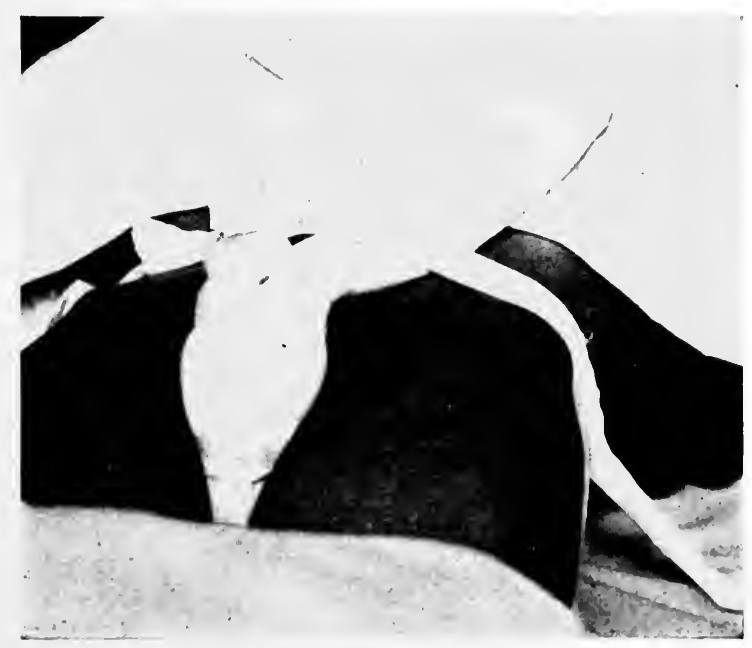

Fig. 46.-Bandage fastened to waist band.

the waistband. The lower edges of the gauze strip are carried tightly around the base of the scrotum and at the root of the penis are pinned together. The same edges, higher up, are pinned to the waistband. The free edges of the gauze strip are then pinned tightly over the scrotum. A tuck is made at the bottom of the scrotum to make the bandage fit better. By drawing upward upon the free edges the whole scrotum 


\section{I74 AN OUTLINE OF GENITO-URINARY SURGERY}

is crowded against the pubes, thereby giving all the compression desired.

Traumatic hydrocele and hematocele may be treated with rest in bed, elevation of the scrotum, and application of ice. Continued pain due to distention of the sac may be met by tapping or by opening and draining the sac; in severe injuries

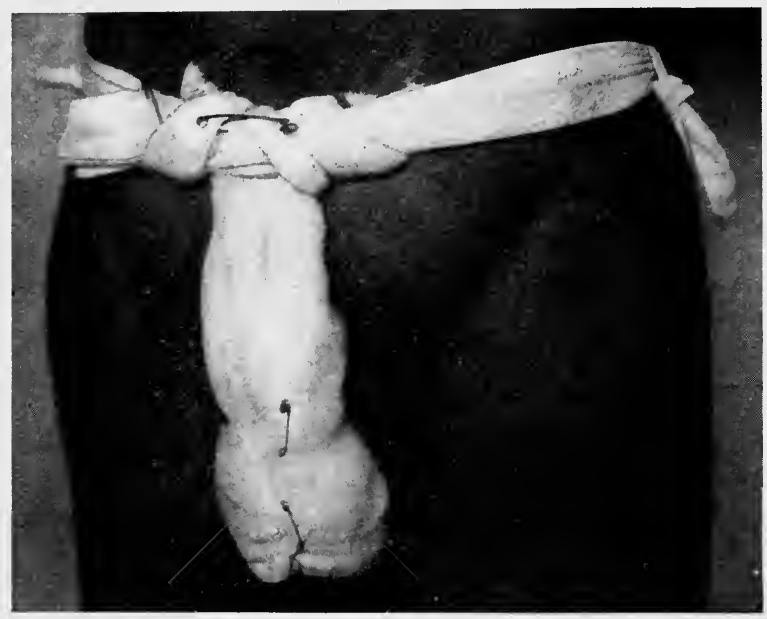

Fig. 47.-Alexander bandage completed. Note how scrotum is pressed against pubes.

the latter is probably the more conservative measure, as extreme pressure may damage the testicle, or the hemorrhage may be due to a rupture of the tunica albuginea, which requires suture.

\section{DISEASES OF THE SPERMATIC CORD}

Hydrocele of the cord is a collection of fluid within an isolated bit of peritoneal sheath which was not completely ob- 
literated after the passage of the testicle. It appears as a smooth, elastic tumor situated in the spermatic cord, somewhere between the testicle and the internal ring. It suggests

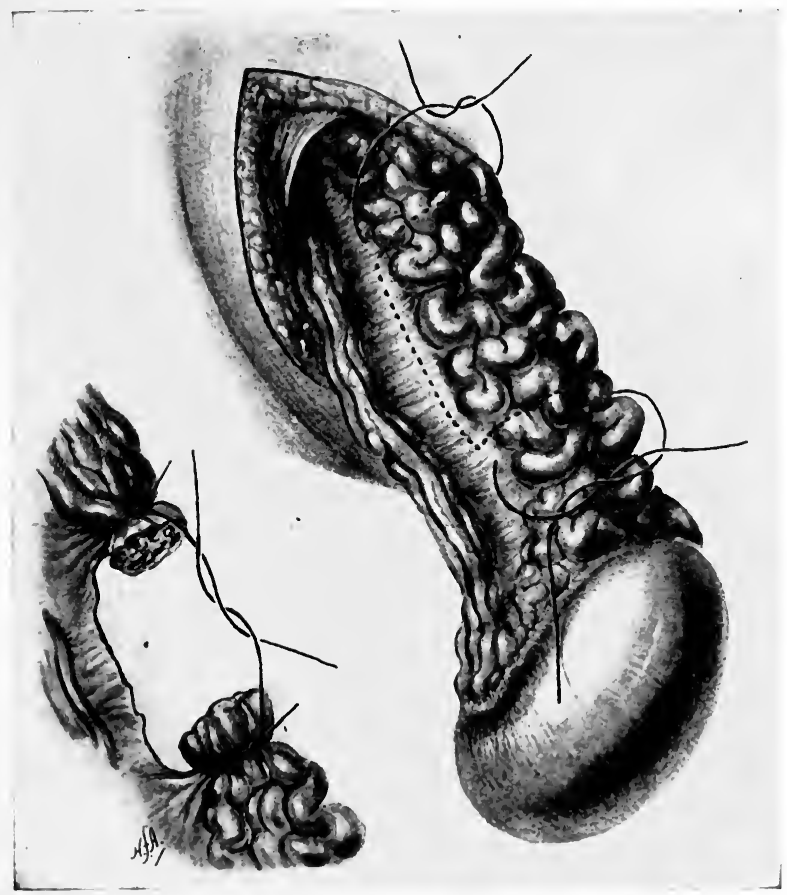

Fig. 48.-Open operation for the radical cure of varicocele. The mass of veins included within the dotted lines is removed and the cut ends are tied together.

hernia, and may be mistaken for it. If troublesome, the sac should be dissected out.

Varicocele is the enlargement of the veins of the pampiniform plexus of the spermatic cord, occurring usually upon the 
left, and most frequently in young men. The condition may or may not cause symptoms; these consist of a dull ache in the region of the scrotum and a dragging sensation. Upon examination, a mass which feels like a "bunch of angleworms" is felt in the upper part of the scrotum.

In very extreme cases it is possible that the nourishment of the testicle is interfered with and atrophy brought about, but in the vast majority of cases the condition is harmless and will clear up when the normal demands of sexual life are provided for. Varicocele may be a manifestation of improper sexual hygiene. Ungratified sexual excitement, if continued for some time, will cause a very painful acute dilatation of the veins of the spermatic cord. Great relief is given by the wearing of a well-fitting suspensory; if the condition is extreme or continues to give symptoms, the excision of the dilated veins may be done, under local anesthesia if so desired. The possibility that a varicocele may be due to the pressure of a renal tumor upon the spermatic vein should be kept in mind.

Torsion of the Spermatic Cord.-Occasionally the testicle rotates upon the pedicle by which it is suspended in the scrotal $\mathrm{sac}$, or, in the case of undescended testicle, in the inguinal canal. The twist is supposed to occur because of an unusually long mesorchium (the mesentery which holds the gland in place and through which the spermatic cord passes). Undescended testicle, because of the abnormal development of its attachments, seems peculiarly liable to this accident. As a result of the twist, which may consist of from one-half 
a turn to four full turns, the circulation of the testicle is cut off and the gland quickly suffers irreparable damage. There is exudation of blood into the tissues; the testis and epididymis become swollen and bluish-black in color. This condition may later resolve into a fibrous atrophy of the testicle, with loss of all its differentiated parenchymal cells, or may cause necrosis and abscess formation within the fibrous envelope.

The symptoms are sudden, severe pain in the scrotum and groin, with swelling of scrotal contents and reddening and edema of the skin. Clinically the condition resembles acute epididymitis so closely that the latter diagnosis is generally made. The absence of pus and shreds from the urine in such a case should make one very cautious of the diagnosis of epididymitis. Under rest and ice the pain and swelling gradually subside, but the testicle remains hard and tender for a number of weeks, and may break down and require removal later.

Operative untwisting of the torsion as early as one and onehalf hours after the onset has not sufficed to save the testicle. Manipulation of the gland might conceivably untwist the torsion. Cases of recurrent torsion have been reported in which the patient himself was able to undo the twist. The extreme tenderness makes manipulation difficult, and if an anesthetic is given the scrotum had better be opened and a thorough inspection made. In case the untwisting of the cord at operation is followed by the restoration of any circulation at all in the testicle, the gland should be left. It will atrophy, but 


\section{I78 AN OUTLINE OF GENITO-URINARY SURGERY}

even so, it will have a certain value in the eyes of the patient. If the case is seen late, or if the testicle is too obviously damaged, orchidectomy should be done. Expectant treatment may succeed in averting an operation, but the patient is liable to be troubled by a tender testicle for several months.

\section{DISEASES OF TESTICLE}

For the diagnosis of diseases of the scrotal contents one must be able to tell whether the disease is in the testis or in the epididymis. Certain diseases attack the former, others the latter. The term "epididymo-orchitis" or "orchitis" has been used far too loosely. Fluid about the testis is the chief obstacle to an accurate diagnosis. In doubtful cases, therefore, it must be withdrawn. The diseases of the testicle propèr are: syphilis, trauma, torsion, new growth, tuberculosis, abscess, and orchitis of mumps and of typhoid fever. Orchitis due to a diffuse infection of low virulence is occasionally seen. The diseases affecting chiefly the epididymis are the infections due to the tubercle bacillus, the gonococcus, and the colon bacillus. Torsion and infection by the typhoid bacillus involve both.

Trauma of the testicle, which will be either a bruise of the gland (treatment, rest and ice) or rupture (blood in tunical sac; treatment, expectant or operative, with suture of the tear), may be diagnosed by the history.

Epididymo-orchitis of mumps comes on during the course of a parotitis. The testicle is swollen and very tender. It is ordinarily treated by rest, elevation, and ice. 
Microscopic examination of tissue taken from a testicle in the acute stage of orchit is of mumps shows "a patchy degeneration of the tubules, with exudation into them of polymorphonuclear leukocytes and phagocytic endothelial leukocytes. The intertubular connective tissue is everywhere edematous, and between the tubules most affected it contains coarse, meshed fibrin, small areas of hemorrhage, and many polymorphonuclear leukocytes and endothelial leukocytes. The picture suggests that the process is extending from the intertubular connective tissue into the tubules." No bacteria or other parasites were found. A late result in the majority of cases is atrophy of the testicle.

Operation for this condition has been done; the tunica albuginea is incised to allow the drainage of the exudate which has been poured out into the testicular tissues. It may be that this procedure, if done early, will prevent to some extent the damage which will otherwise almost certainly result. The evidence upon this point is lacking. Following operation the pain is quickly alleviated, which suggests that the intracapsular pressure has been relieved.

Epididymo-orchitis of Mumps-Operation.-F. C., age twenty-two, cashier, single. Never sick except for measles. Denies venereal infection. July 1, 1912, the right parotid began to swell and be painful. $A$ few days later the left side followed suit. July 9 th the left testicle became sensitive and began to swell, and for two days got progressively worse. There was no burning on urination.

My first examination, July 11th, showed a flushed, well-developed young man, with considerable swelling of both parotids. Thorax and abdomen normal. No urethral discharge. Right testicle normal. Scrotum contracted, not reddened. Left testicle was three times as large as its fellow, hard, smooth, only moderately tender. No hydrocele, skin 
not adherent. Epididymis and vas not abnormal. Rectal examination showed a soft, small prostate, not tender. Vesicles not indurated. Temperature $104^{\circ}$ F. Pulse 104. Respiration 26. He was sent to the Baptist Hospital, and flaxseed poultices applied.

July 12th: Skin of scrotum red. Some hydrocele present. Testicle was larger and more tender. The epididymis had become palpable. Operation advised, but rejected. July 13th: Testicle felt a bit less swollen. Hydrocele had increased. Epididymis was more clearly involved. The patient requested operation, which was done under ether, after soap and water and alcohol preparation.

Operation.-A 2-inch vertical incision along anterior aspect of left scrotum. Tunica vaginalis opened, with escape of about 1 ounce of turbid yellow fluid from which a culture was made. ${ }^{1}$ The testicle was delivered through this incision. It was three times the size of a normal testicle, firm and elastic on palpation. The color was more bluish than is usual, and throughout the tunica albuginea were scattered many minute reddish specks, probably punctate hemorrhages. The epididymis was definitely enlarged, soft, without induration, and of a deep red color, which at the globus major became almost black. It was the picture of intense acute congestion. The cord was somewhat edematous, the vas normal. The tunica albuginea was slit with the knife in a dozen places, the incisions being not over $\frac{1}{4}$ inch long, parallel to the long axis of the testicle, and extending just through the tunica. There was considerable ooze from these, lasting a few moments; one spurter required a ligature. The testicular tissue showed no tendency to extrude. The tunica covering the epididymis was also scored in about six places.

The testicle was replaced in the scrotum, a rubber tissue drain left in, and the scrotal wound closed about the drain by a subcuticular silkwormgut suture. Scrotum was tightly compressed in an Alexander bandage.

July 14th: Temperature normal. No pain since operation. July 15th: Same. Urine turbid, high colored, containing very slight trace of albumin. No sugar. Sediment: Some pus.

July 16th: Drain removed. Testicle of normal size. Only slightly tender.

July 17th: Up and about. Urine still contains pus.

July 19th: Discharged from the hospital.

July 20th: Stitch removed. Testicles of same size. Urine sparklingly clear, no albumin.

${ }^{1}$ No growth, although a number of media were used. Monkeys were inoculated by injecting this fluid into the testicle, but orchitis did not result. 
Abscess of the testicle presents the signs of inflammation added to the testicular enlargement. It occurs almost always as an accompaniment of colon infections of the lower urinary tract, and is most often found in old men with prostatic obstruction. A pyogenic infection of the testicle characterized by areas of focal necrosis, the so-called "Kocher's testicle," may be an early stage of abscess of the testicle. In such cases the testicle is enlarged, firm, and tender. Orchid- . ectomy is the best procedure.

Abscess of the testicle must be treated by incision and drainage or by castration. As a rule the entire contents of the testicular envelope become necrotic, so that castration does not deprive the individual of anything of value.

Abscess of the Testicle.-C. J. J., age thirty-nine, single, entered the hospital October 3, 1914. He denied venereal disease and there was nothing of importance in his past history, except a street car accident five years previously, at which time his right leg was cut and bruised and he was obliged to walk on crutches for thirteen weeks. Eight months later he noticed that his left testicle was swollen. He went to the City Hospital, where the testicle was "lanced," with the escape of considerable pus. A few days later the testicle was removed. Since that time he had had no trouble and had felt well and strong until seven weeks ago, when he began to have pain in right testicle, but did not notice any change in size at that time. A few days later, however, it began to trouble him as he moved about at his work, and he noticed a slight increase in size, also a hard, slightly tender cord in the inguinal region extending into the scrotum. At that time he felt "run down"; lost his appetite; was nauseated, and felt dizzy at times. This condition lasted only a few days, and since then he has felt perfectly well. The testicle, however, has continued to enlarge and has been slightly tender.

Examination showed a healthy looking man. The left testicle was missing; the right was enlarged to equal a good-sized egg, smooth, and quite tender. Skin of scrotum not adherent, but reddened. Examination of urine showed a few pus-cells, rare cast, and red cells. 


\section{I82 AN OUTLINE OF GENITO-URINARY SURGERY}

October 5, 1914, operation, gas-oxygen anesthesia. A 3-inch vertical incision was made in anterior surface of scrotum and some hydrocele fluid evacuated. Testicle fairly adherent to tunica. On freeing the testicle it was found to be congested, elastic, and much enlarged, but of normal appearance. No nodules could be felt. Epididymis was flat, much congested, but not indurated. A small incision in the upper pole of the testicle set free about 6 drams of thick, yellow pus. When all the pus was evacuated nothing was left but a thin rim of testicular tissue. A rubber tube was passed through the testicle and out through a counteropening in the lower pole of the testicle and scrotum.

A culture of pus from the abscess showed colon-like bacilli.

Convalescence was uninterrupted and the patient left the hospital October 14, 1914.

Tuberculosis of the testis is always secondary to a similar process in the epididymis, but is by no means a frequent complication. In cases of long standing the epididymis may have sloughed away, leaving a tuberculous testicle. In such a case the chronicity and the presence of sinuses make the diagnosis of tuberculosis almost certain.

Syphilis of the Testicle.-A non-tender enlargement of the testicle is due to one of two causes-syphilis or tumor. The differential diagnosis is not easy. A total lack of sensibility in the gland suggests syphilis, and, of course, a positive Wassermann tends to confirm this diagnosis.

In every case in which the diagnosis between these two conditions is not perfectly clear, castration should be done. A gummatous testicle is not much of a loss, whereas the postponement of removal of a malignant testicle may be fatal.

A syphilitic testicle may be so improved by antispecific treatment that it feels normal to palpation, although its functional recovery is improbable. Spermatozoa have been 
recovered from healed leutic testes. If the gummata are broken down, castration will do away with that lengthy process of disintegration through which the testicle must go.

Neoplasm of the Testicle.-As has been shown by Ewing, practically all new growths of the testicle are teratomasmixed turnors composed both of sarcomatous and carcinomatous elements. These tumors are quick to form metastases, which occur at first in the lymphatic glands of the iliac and lumbar retroperitoneal region. Castration as high as possible should be done immediately after the diagnosis is made. Postoperative treatment of the abdomen by $x$-ray with the hope of destroying metastatic growths should be carried out. Hinman points out that simple orchidectomy has resulted in cure in only 15 to 20 per cent. of the cases operated upon at Johns Hopkins. He therefore advises the removal of all retroperitoneal lymph-glands on the affected side between the renal vessels and the inguinal canal. This should not be attempted, he says, if the glands in question are sufficiently involved to be palpated through the abdominal wall. The mortality of this procedure, in 46 cases reported in the literature, was I I per cent. The operation is perfectly logical, but it seems too extensive for the rather douttful benefits which it might yield. It should be kept in mind, however, for use in selected cases.

Epididymitis.-Inflammation of the appendage of the testicle is a distinct disease entity, and only rarely is it accompanied by corresponding disease of the testicle. Torsion and typhoid fever involve both; advanced tuberculosis 


\section{I84 AN OUTLINE OF GENITO-URINARY SURGERY}

may involve both. In the vast majority of cases of disease of the epididymis the lesion affects only that structure itself, perhaps being prevented from entering the testicle by the stout envelope which envelops the gland. Under such circumstances the normal condition of the testicle is evident upon palpation, whereas the epididymis presents a hard, tender, crescentic tumor upon the posterior aspect of the testis. The globus major and globus minor are especially prominent. The vas is generally thickened. Differentiation of the scrotal organs may be obscured by fluid in the tunica; if the diagnosis is in doubt, this should be drawn off in order to make possible thorough palpation of the scrotal contents.

The three infections commonly causing epididymitis are those due to the tubercle bacillus, the gonococcus, and the colon bacillus. Tuberculosis gives a hard, smooth, only slightly tender enlargement of the epididymis, limited sometimes to one pole. The onset is insidious, or may be attributed to some trivial injury. The urine may be normal or may show the signs of infection by the tubercle bacillus. If the latter, it will be hazy with pus, in a specimen of which, stained simply with methylene-blue, none of the ordinary bacteria appear. The prostate may be normal or may contain nodules; the seminal vesicle upon the infected side, however, is very likely to be thickened and knotted. The vas in its scrotal course feels beaded, and as it approaches the globus minor it becomes thicker. The skin over the epididymis later becomes adherent, and the presence of sinuses is 
almost pathognomonic of tuberculosis. A subsiding epididymitis due to the gonococcus or colon bacillus may resemble a tuberculous epididymitis. Chronic epididymitis, with mild exacerbations, is also puzzling. The history of urethritis or of an acute onset of the epididymitis weighs strongly against tuberculosis, although the possibility of the latter being superimposed upon a gonococcus epididymitis must be considered.

Epididymitis caused by the gonococcus and that due to the colon bacillus present the same symptoms. Both are acute infections. The onset is rapid and painful, the scrotum is at first tightly contracted, but quickly becomes edematous, red, and relaxed. After twenty-four hours the swelling of the epididymis becomes apparent, and reaches its maximum about the fourth day. It then becomes harder, but less painful, and from that point the enlargement gradually subsides. Sections of an acutely inflamed epididymis show the canals choked with detritus and pus-cells; the tissue between the tubules is edematous and contains collections of leukocytes. Macroscopically, the epididymis is purplish in color and perhaps coated with fibrin. The fibrous sheath is distended, and through it tiny yellow collections of pus occasionally appear. Puncture of this sheath is followed by free oozing from the congested vessels, and perhaps by the extrusion of a drop of pus.

The differential diagnosis between epididymitis of colon origin and that caused by the gonococcus depends upon the history and upon the condition of the urine. Shreds in the urine and the history of a urethral infection point to the 
gonococcus as the etiologic factor; the presence of residual urine, vesical tumor, calculus, or colon bacilluria suggests the colon bacillus.

The treatment of tuberculous epididymitis will be considered in the chapter on Genito-urinary Tuberculosis.

The treatment of acute epididymitis consists of (I) support; (2) heat. Rest in bed, with a flaxseed poultice and an efficient bandage, such as the Alexander bandage, gives good results. Elevation of the scrotum is essential. With such treatment the inflammation should quiet down in from five to ten days, but there is always the possibility that the epididymitis will flare up again when the patient gets out of bed. When ambulatory, the sufferer from epididymitis will get a good deal of relief from wearing an elastic athletic supporter. The application of various drugs, such as magnesium sulphate and ichthyol, has a very doubtful value. If one really wishes to clean up an acute gonococcus epididymitis without fear of recurrence, or if one has a case which has already suffered recurrences, the best treatment is the operation of epididymotomy. Under local or general anesthesia the testicle is exposed by a vertical incision. on the anterior surface of the scrotum, is turned out of the scrotum, and the epididymis punctured in many places with a fine tenotome. Small abscesses are evacuated; the testicle is replaced in the scrotum and the skin incision is closed with silkworm-gut sutures. A rubber-tissue drain is left in the tunical sac. This operation is practically certain to cure the condition within one week, and can be done under local 
anesthesia with very little pain. Expectant treatment may do as well, but cannot be depended upon to do so.

Barney, J. D.: Abscess of the Testicle, Trans. of Amer. Assoc. Genitourin. Surg., 1913, viii, 163-174.

Ewixg, J.: Teratoma Testis and its Derivatives, Surg., Gynec., and Obst., 1911, xii, 230-259.

Hagier, F. R.: A Further Report on the Operative Treatment of Acute Gonorrheal Epididymitis, Ann. Surg., 1908, xlviii, 878-882.

Hismax, F.: The Operative Treatment of Tumors of the Testicle, with Report of 30 Cases Treated by Orchidectomy, Jour. Amer. Med. Assoc., 1914, lxiii, 2009-2015.

RigBY and Howard: Torsion of the Testis, London Lancet, 1909, i, $1415-1421$.

Sмiтh, G. (i.: Two Cases of Orchitis Due to Mumps Treated by Operation, Boston Med. and Surg. Jour., 1912, clxvii, 323-325. 


\section{CHAPTER XII}

\section{DISEASES OF THE BLADDER}

A THOROUGH understanding of the diseases of the bladder is the key to diagnosis in urology. The bladder is the indicator for the entire tract. Like most generalities, this statement is only partially true, but it is true in a large enough proportion of genito-urinary diseases to be allowed to pass unchallenged. If the significance of the bladder symptoms is clearly appreciated, one can often go a considerable distance toward diagnosing the underlying lesion. The great mistake is to treat all cases of pyuria as "cystitis," and in such cases to wash the bladder regularly for months when the trouble is really primary in the kidney or in the urethra.

The bladder has but one way of voicing its dissatisfaction with existing conditions, and that is through some disturbance of micturition. Frequent micturition, however, may be due to any one of several different causes. A contracted bladder, the capacity of which is really limited by scar tissue, must be emptied more often than one the natural elasticity of which is unimpaired. Again, the bladder itself may be normal, but the posterior urethra may be hypersensitive. Stimuli to urination which would be disregarded by the normal urethra are able to arouse the urinary reflex. There is 
the frequency of the "overflow" bladder, caused by the inability of the viscus to expel more than a few drams or ounces at each attempt. Or frequency may originate wholly in the nervous system, sometimes from functional derangement, sometimes from organic disease. Painful urination is a further development along the same line, and usually indicates a definite lesion of the bladder neck, such as ulceration of the mucosa.

By far the commonest cause of bladder symptoms is infection. Disturbance of circulation, causing congestion of the posterior urethra or trigone, is a less frequent cause, unless we include cases of urinary frequency in pregnant women. I believe that in many cases of supposedly aseptic congestion an underlying infection of urethral glands or prostate is the true cause. Of course, there are other causes of pain. Nerve lesions, such as pressure upon the roots of the sensory nerves of the bladder by spinal deformities or by metastases from cancer, may give vesical symptoms. Stone in the kidney or ureter may be the cause of irritability of the bladder even when infection is not present. This may be due to the existence of a renovesical reflex, although definite proof of such a reflex has not been presented. Vesical calculus, even when unaccompanied by infection, is, of course, likely to cause pain in the bladder by purely mechanical means.

Cystitis.-Infection, however, is the great factor. In the vast majority of cases the organism is the colon bacillus; at times it is the staphylococcus or streptococcus, and not in- 
frequently the tubercle bacillus. Infection by other organisms is unusual, though not by any means rare.

Practically never do we find an infection of the bladder without underlying cause. Acute infections may attend the sudden emptying by catheterization of overdistended bladders; this occurs most frequently when the traumatized bladder of the puerperium is allowed to distend and is then emptied at one catheterization. Acute infection of the trigonal portion of the bladder occurs during urethral infections by the gonococcus. In the normal bladder such acute infections clear up quickly. A chronic cystitis means either (I) a constant supply of infectious material from some focus, such as kidney, prostate, or more rarely an appendix abscess or an inflamed fallopian tube adherent to the vesical wall; (2) the presence of residual urine, due to the inability of the bladder to empty itself. The cause of this may be in the reflex arc, as in tabes dorsalis or other cord lesion; a mechanical obstruction, as in cystocele, obstructing prostate or stricture; a diverticulitis, with retention of urine in the diverticulum. Or (3) cystitis may be due to a loss of the integrity of the bladder mucosa, as in ulceration due to syphilis, to stone, or to tumor.

Differential Diagnosis.-How may these conditions be differentiated, and what can be done, short of cystoscopy, to work out the problem here presented? Unless the diagnosis is accurately made, treatment can be but symptomatic, and in too many cases will result in dissatisfaction and perhaps in wasted opportunities. 
The procedure to follow is simple enough. Given a case oi acute cystitis from any cause, the first step is to abate the discomfort and, if possible, allay the virulence of the infection.

Rest in bed, forced fluids, a bland diet-cereals, milk, and eggs-and free catharsis are the foundation for the treatment. Water, hot or cold, carbonated or plain, I00 to 150 ounces a day, is absolutely essential. As an additional measure, one may give an alkali, preferably potassium citrate, combined with a bladder sedative:

$$
\begin{aligned}
& \text { R. Potassium citrate........... } 3 \mathrm{v} \\
& \text { Tincture hyoscyamus......... } 3 \mathrm{v}
\end{aligned}
$$

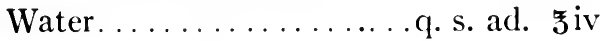

A teaspoonful may be given in half a glass of water every three hours.

Sandalwood oil (ro minims in capsules three or four times a day) is extremely soothing and of decided value in decreasing bacterial activity.

The kidneys should always be palpated to determine the presence of tenderness, abnormal position, or enlargement. In the male a rectal examination should be made to determine whether the prostate is concerned; in the female, vaginal examination is not so likely to yield information.

In acute infections confined to the bladder the temperature should not be elevated more than a degree at most. Fever means infection of a parenchymatous organ, such as kidney or prostate. 


\section{I92 AN OUTLINE OF GENITO-URINARY SURGERY}

The urine must, of course, be examined. In the male catheterization is not necessary if the examination be done within an hour after the passage of the urine; in the female contamination by vaginal secretion really nullifies the findings in urine voided naturally. In women, a catheter specimen is essential.

The reaction of the urine is worth nothing. Tuberculous and colon infections are acid; if the urine is alkaline (before the administration of an alkali) an acute exacerbation of a chronic infection due to retention is suggested. The general characteristics of the urine should be noted. An ammoniacal odor means retention, either in bladder or in kidney pelvis. A smoky color means blood. Haziness without shreds suggests renal origin; shreds suggest a prostatic origin. Å heavy deposit of pus suggests pyonephrosis.

The diagnosis cannot be made by the finding of any one of the above characteristics, but the direction of future investigations may be so indicated. The sediment should be examined for (I) blood, (2) pus. The detection of special cells from various portions of the tract is of some value. The cell which gives the most information is the caudate cell of the renal pelvis, the presence of which, of course, suggests a renal origin for the infection.

The sediment should then be examined for bacteria. This procedure, described in Chapter II, will show whether the infectious agent is the colon bacillus or a coccus; the absence of ordinary bacteria in the presence of pus strongly suggests tuberculosis. If the latter is suspected, a specimen 
of urine obtained by catheter to avoid contamination by the smegma bacillus should be sent to someone experienced in the recognition of the organism, either in smears or by guineapig inoculation. The latter takes from five to six weeks, but the accuracy of the test makes the delay worth while in case the tubercle bacillus is not found in smears.

Lavage of the bladder in acute cystitis need not be done, unless there are indications of the presence of a residual urine of several ounces or more. In the latter case lavage with boric acid ( 2 per cent.) will quickly reduce the inflammation. Daily instillations of argyrol (ro per cent.) may be used, but are not necessary.

As the acuteness of the infection wears away, as it should in three or four days, hexamethylenamin may be substituted for the sandalwood oil, if the infection is due to the colon bacillus. From 30 to 45 grains a day should be given; as it yields formalin only in acid urine, the alkali should, of course, be discontinued. If the bladder is not sensitive the urine may be rendered more acid by the administration of sodium acid phosphate, ro grains three or four times a day. Increase of bladder irritability means too much formalin in the urine; diarrhea may be caused by the sodium acid phosphate.

In urinary tuberculosis hexamethylenamin acts only as an irritant, and has no curative effect.

If the cystitis clears up entirely and the urine becomes free from pus, the administration of an antiseptic should be continued for two weeks or a month. Two weeks after its discontinuance a catheter specimen of urine should be cultured, 


\section{I94 \\ AN OUTLINE OF GENITO-URINARY SURGERY}

to make certain that there is not a residual bacilluria. Such a bacilluria will probably be of renal origin, and may cause pyelonephritis in case the patient, if a woman, becomes pregnant, or if a man, in case his resistance falls below par.

If the signs of urinary infection persist, one may determine with considerable accuracy whether or not the focus is above the bladder by the following procedure: Wash the bladder thoroughly with boric acid solution until the fluid returns absolutely clear. The catheter is then left in place for a few minutes and the urine collected as it accumulates in the base of the bladder. If the infection is renal the urine should show about as much pus as did the bladder specimen; if the infection is not renal the urine so obtained should be very nearly normal-much more so than the bladder specimen.

The information so obtained is not of great value, however, as it does not disclose the nature of the renal or the vesical lesion. The fact that there is infection in the kidney does not rule out a vesical lesion. On the contrary, the renal infection may be merely incidental to some pathologic condition in the bladder. Infection in the bladder does not ascend the ureter through a normal ureteral orifice; the valvular formation prevents this. If the ureteral valve is dilated by back pressure, as in a chronically overdistended bladder, or the outflow of urine from the ureter is hindered by the presence of a diverticulum or the growth of a tumor, infection of the corresponding kidney soon follows. 
When cystitis is secondary to renal infection the cure of the latter will nearly always result in recovery of the former. If a low-grade chronic cystitis persists, it may be cleaned up by a few irrigations with silver nitrate solution ( $1: 5000$ ). Tuberculous cystitis is an exception to the above rule, although even it shows a general tendency to follow the same principle.

Encrusted Bladder.-There is one type of cystitis sometimes due to renal infection which requires special discussion. It is the form in which lime salts are deposited by an alkaline urine upon the bladder wall. So-called "encrusted bladkler" occurs oftenest, perhaps, in the presence of a residual urine. It may occur in bladders which empty completely, and be caused by a coccus infection originating in the kidney.

The treatment of such cases consists in keeping the urine acid, and instilling into the bladder every day or two 2 ounces of a vigorous culture of lactic acid bacilli, or the Bacillus acidophilus (Caulk). A very obstinate case which we treated at the Massachusetts General Hospital showed no real improvement until, probably as the result of ureteral catheterization, a colon bacillus infection was engrafted on to the coccus infection of the renal pelvis. The reaction of the urine then became acid and the bladder cleared up rapidly.

Case of Encrusted Bladder.-C. L. E. No. 209,515. M. G. H., colored, age twenty-nine, married, came to Out-patient Department complaining of dysuria and passing of gravel with urine. Duration of this six years. Previous history unimportant. During past few years she 


\section{I96 AN OUTLINE OF GENITO-URINARY SURGERY}

has had attacks of "gastritis," manifested by epigastric pain, nausea, and vomiting. General examination negative. The urine was strongly alkaline and ammoniacal and contained considerable pus and red blood-cells. At cystoscopy the bladder held only 4 ounces. Over the left lateral wall were seen masses of white encrustation. Ureters could not be identified. She was given bladder lavage and several instillations of Bacillus bulgaricus. Twelve days later the bladder held 7 ounces, but otherwise showed little improvement. Ureters were identified as round holes, not retracted. Both were catheterized. The right side yielded normal urine; that from the left contained pus and streptococci. No tubercle bacilli found. $x$-Rays negative for stone. Wassermann negative. Treatment in the Out-patient Department was not followed by much improvement, so one month after her first visit she was sent into the House. There she was kept in bed, given daily bladder irrigations, and instillations of either Bacillus bulgaricus or Bacillus acidophilus twice a day. Cystoscopy after ten days of this showed that encrustations had entirely disappeared. Ureteral orifices appeared normal. Both catheterized, with good flow from both kidneys. Right side showed pus and blood, 100 leukocytes per field; colon bacilli; phthalein output after intravenous injection-appearance time three minutes; 7.5 per cent. excreted in following fifteen minutes. Left side showed pus, 50 leukocytes per field, colon bacilli, and streptococci. Appearance of phthalein, three and onehalf minutes, 1.25 per cent. in fifteen minutes. For eight days after cystoscopy the patient ran a temperature of $101^{\circ} \mathrm{F}$. This fell to normal, and eleven days after cystoscopy she was discharged from the house, having recovered entircly from her dysuria and the passage of gravel. The urine remained acid.

Chronic cystitis is always the result of some definite pathologic process. It must be borne in mind that the symptoms of frequency and urgency do not warrant the diagnosis of cystitis. Especially in women, these symptoms frequently exist in connection with a chronic urethritis. Cystitis means inflammation of the bladder, and pus must be found in a catheter specimen of urine before the diagnosis is justified. The situation in regard to cystitis may be diagrammatically presented thus: Chronic cystitis = infection caused from 
I. Renal and ureteral infections, due to
A. Calculus.
B. Stricture of ureter.
C. Renal retention.
D. Renal tumor.
E. Chronic pyelitis or pyelonephritis.

II. Residual urine in bladder, due to
A. Nerve lesions.
B. Prostatic obstruction.
C. Diverticulum of bladder.
D. Cystocele.
E. Stricture of urethra,
F. Tumor or stone acting as ball-valve.

III. Adjacent foci which communicate with bladder:
A. Enterovesical fistula.
B. Pelvic abscess (appendix or tubes).
C. Prostate or vesicles.

IV. Ulcerations of vesical mucosa, caused by
A. Syphilis.
B. New growth.
C. Calculus.
D. Foreign body.

The differential diagnosis between these various causes of chronic cystitis ultimately depends upon the cystoscope. Without it, even if a possible cause is found, as, for example, a pelvic abscess, one cannot be sure that the cystitis is not due to a coincident but entirely independent cause, such as a neoplasm. A true diagnosis is difficult enough to make, 


\section{I98 AN OUTLINE OF GENITO-URINARY SURGERY}

at times, with all the facilities which we command. No case of chronic cystitis should be permitted to continue, after a therapeutic attack of reasonable length, without a cystoscopic examination.

Once the diagnosis is made, the treatment of the cystitis is the treatment of its cause. Frequently this cause has to do with other organs: the treatment will be found in the appropriate sections of this book. Pathologic conditions concerning chiefly the bladder and not described elsewhere are disturbances due to nerve lesions, syphilis, bilharzia infection, neoplasm, and stone.

A study of 87 cases of "cystitis" in women thoroughly investigated by modern methods of diagnosis showed that the bladder infection was associated with:

Renal infections, not tuberculous, in 6r per cent.

Renal tuberculosis in I9 per cent.

Incomplete emptying of bladder in 7 per cent.

Systemic and pelvic infections in 7 per cent.

Other causes in 6 per cent.

Not one case of chronic cystitis was found in which the process was not dependent upon some underlying cause (G. G. Smith).

Residuum due to nerve lesions is most frequently found in the tabetic. The reflex arc is broken by the involvement of the roots of those sensory nerves which bring from the bladder neck the sensation of a desire to urinate. Instead of regularly emptying the bladder, the tabetic voids at irregular intervals, and may be totally unconscious of the fact that when he 
finishes urinating his bladder still contains 3 or 4 pints of urine. The muscle of the bladder wall strives valiantly to express the contents; it is opposed by the sphincter, which awaits in vain the signal to relax. The bladder wall becomes hypertrophied and the muscles develop in bundles; with the cystoscope this is shown by the trabeculation of the vesical wall. These bladders seem to be immune to infection to a remarkable degree. Sooner or later, however, it intervenes, and, as has been shown by Barney, death is caused in a majority of tabetics by damage done to the kidneys through back pressure and infection.

Treatment.-To avoid this termination, the aim of treatment must be the complete emptying of the bladder. The residuum must be emptied by catheter. Instrumentation is easy, as it is unusual to have troublesome enlargement of the prostate as a complication of tabes dorsalis. The combination does occur, and for that reason every case of residual urine should be examined with regard to the four chief reflex tests for nerve disturbance, $i$. e., pupillary reaction, kneejerks, ankle-jerks, Romberg.

The frequency of catheterization should depend upon the amount of residuum. From 2 to 4 ounces require the catheter once a day; 4 to 8 ounces, twice daily; more than that requires regular catheterization three or four times in the twenty-four hours.

Hexamethylenamin in efficient doses should be given, and the urine kept acid by acid sodium phosphate. If in spite of this the urine remains alkaline, the instillation into the 
bladder every day or two of an ounce of lactic acid bacilli in sterile water may be tried. The treatment of locomotor ataxia itself is not within the scope of this book. It is worth while, however, to mention the value of re-education of muscles as applied to the bladder. Filling the patient's bladder with fluid, and, as he voids this, making him stop and start the stream at the word of command, may enable the patient to get control over his external sphincter, so that it makes an acceptable substitute for the internal sphincter.

Other and less well-recognized nerve lesions are those due to the effects of diphtheria toxin, which may be manifested years after the attack of diphtheria, those due to pressure upon the cord or to involvement of the cauda equinæ, as in Pott's disease, or in spina bifida and spina bifida occulta, and those due to syringomyelia, transverse myelitis, and multiple sclerosis.

Bladder Disturbance Due to Syringomyelia.-A patient with syringomyelia exhibiting marked bladder symptoms entered the Massachusetts General Hospital on the genito-urinary service in October, 1916. The relation between the vesical disturbance and the nerve lesion was not perfectly clear, yet we were unable to account for the former in any other way. The patient was a woman of thirty, a domestic who had had three illegitimate children. Two of them were born dead; the last one, which lived, was born five years before. The patient complained of painful, burning micturition of eight weeks' duration. Urination was followed by dribbling, tenesmus, and a desire to urinate again. The urine contained pus and colon bacilli. Cystoscopy was done under spinal anesthesia. Considerable trabeculation of the bladder wall with a distention of 6 ounces was noted. Both ureters were catheterized; a small amount of pus and a growth of colon bacilli were obtained from each. Roentgenoscopy and the Wassermann test were negative. The peculiar thing was that during her twelve days in the hospital the patient continued to have constant severe spasms of the bladder in spite of every kind of local 
treatment. Also, she had, on several separate observations, a residuum of 6 ounces.

She was seen in consultation by Irs. Paul and Taylor, who made the diagnosis of syringomyelia because of atrophy of the small muscles of the hands and various sensory disturbances.

Bladder Disturbance Due to Multiple Sclerosis.-M. C., a nurse, aged twenty-eight, was seen about one year ago. Four years before, while in training, she became "run down" and very nervous. Previously she had always been well, except for measles. Accompanying her breakdown were bladder symptoms-great frequency and inability to control her urine. Association with insane persons led her to fear that she might have some nervous disease, so she went to the Massachusetts General Hospital and had an examination of the spinal fluid. This was normal. She returned to work and has remained at it, in spite of a sense of weakness in the legs, and what she characterizes as a "weakness of the bladder." At times she wets the bed at night.

She was a rather pale, tired-looking girl. The pupils reacted normally. There was no Romberg; the knee-jerks were normal; general examination was negative; the kidneys were not palpable; the pelvic organs were normal. The urine by catheter was hazy. revealing no sugar or albumin. A centrifuged sediment showed some pus-cells, a few red bloodcells, and vast quantities of staphylococci. No tubercle bacilli were found. Roentgenoscopy was negative for stone.

The bladder contained 4 ounces of residual urine; with distention of 8 ounces the bladder wall showed a fine trabeculation; the mucous membrane was clean. The ureteral orifices were normal. Both ureters were catheterized, with a flow of normal urine.

Not being awake to the diagnosis, and finding the urethra abnormally red and granular, I treated her with urethral applications, sandalwood oil and bladder lavage. 'The residual urine remained about 4 ounces throughout July, August, and September. In November I noted that her chief trouble was a sensation of weakness in the front of the legs. The bladder symptoms improved a very little, the greatest improvement following rest from work, bromids, and belladonna.

The patient was very emotional, laughing one moment, crying the next. On December $22 \mathrm{~d}$ the residuum was only $1:$ ounces. In Iecember I sent her to Dr. George Clymer for neurologic examination. He reported that he found a positive Babinski, negative abdominal and epigastric reflexes, and positive ankle-clonus-in short, that she unquestionably had multiple sclerosis.

He started treatment with solution of potassium arsenite (Fowler's solution) and the application of electricity over the bladder. Whether or 
not as a result of this treatment, the patient showed remarkable improvement. The residuum cleared up, the urine became normal, and for two months she wet the bed only during menstruation. She has had a slight remission, but, generally speaking, is much better, and has started to work again.

Although these are unusual causes of urinary retention, they should be borne in mind whenever one is searching for

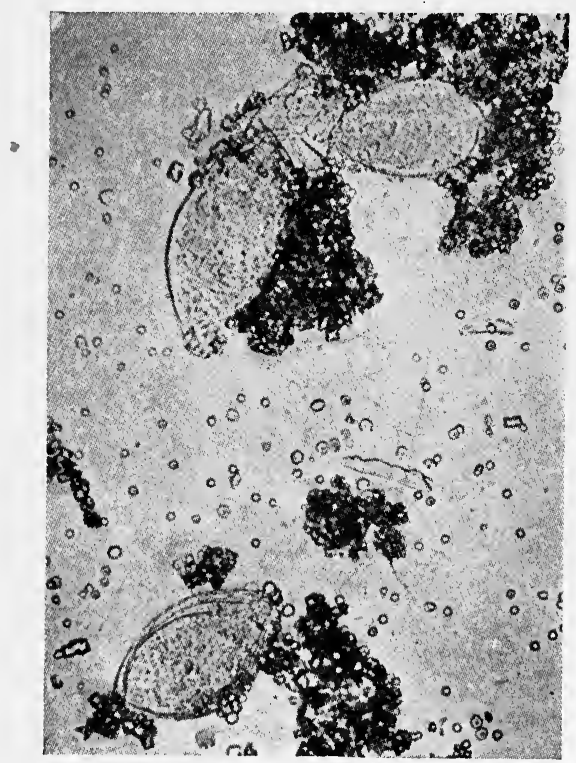

Fig. 49.-Bilharzia ova in urine. Lower power of urinary sediment, showing blood, pus, crystals, and ova. In upper right-hand corner is miracidium that has just hatched out, leaving the empty shell behind it (to left); $\times 200$ (Lane).

the cause of residual urine. Multiple sclerosis occurs in young women especially, and the detection of this condition may explain an otherwise inexplicable residual urine.

Another factor in disturbance of the bladder innervation 
has been pointed out by Chute. Frank spina bifida is a wellrecognized cause of bladder paralysis, but Chute has reported several cases of spina bifida occulta, in which the $x$-ray revealed a defect in the wall of the vertebral canal, and in which there was partial or complete retention of urine.

Syphilis of the bladder is of rather infrequent occurrence. At least the recognized cases are few. There is nothing

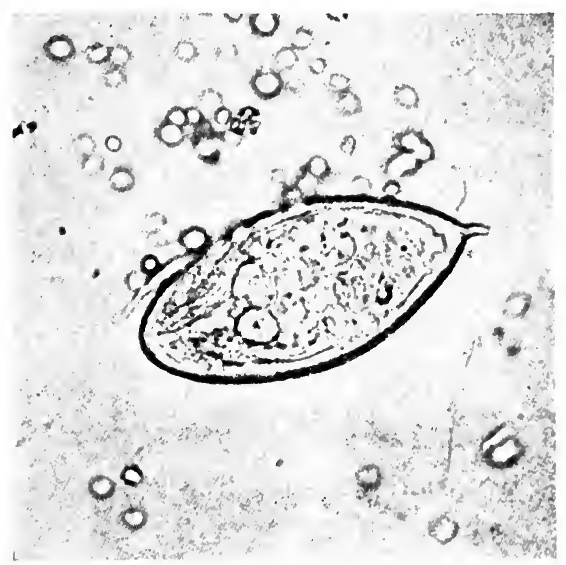

Fig. 50.-High power of ovum showing structure; $\times .375$ (Lane).

especially typical about the lesion. It consists of an ulceration of the bladder wall which does not respond to lavage, but which quickly disappears under antispecific treatment.

Bilharzia infection is worth mentioning because it is occasionally met with in our hospital clinics. E. L. Young had 2 cases in one year. The symptoms are similar to those of bladder tumor, and the appearance of the lesion is not unlike that of an infiltrating, solid growth. The finding 
204 AN OUTLINE OF GENITO-URINARY SURGERY

of the ova in the urine is, of course, the essential factor in diagnosis,

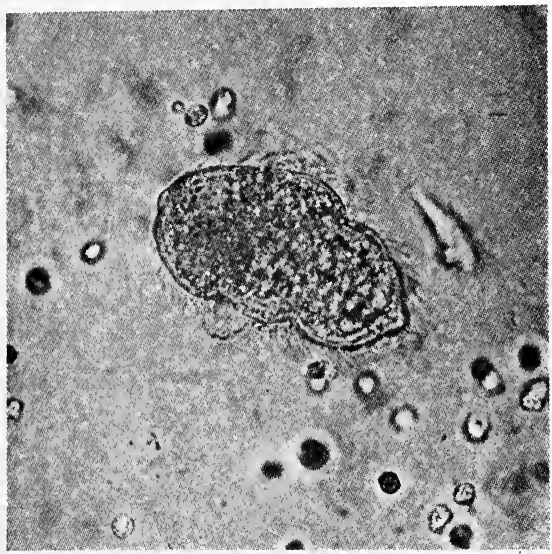

Fig. 51.-Represents miracidium swimming about, showing the different forms assumed; $\times 375$ (Lane).

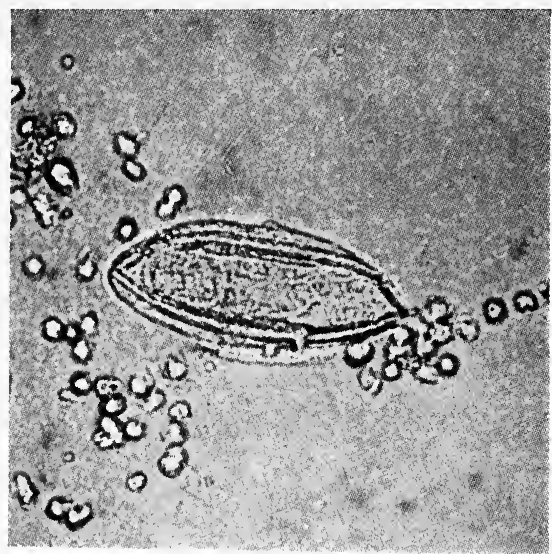

Fig. 52.-Empty ovum after escape of miracidium, $\times 375$ (Lane).

Tumor.-Bladder tumors are either benign or malignant. The common benign tumor is the papilloma; the rarer benign 
tumors are those composed of fibrous, smooth muscle or mucilaginous tissue. Malignant bladder tumors are either papillary carcinoma (papillomata which have undergone malignant degeneration), squamous-celled carcinoma, or sarcoma. Carcinomata of the epithelial type are meaty, solid growths, sessile in form, or are ulcerative in character. For practical purposes all papillomata are malignant, inasmuch as they all tend to recur. Even when the original

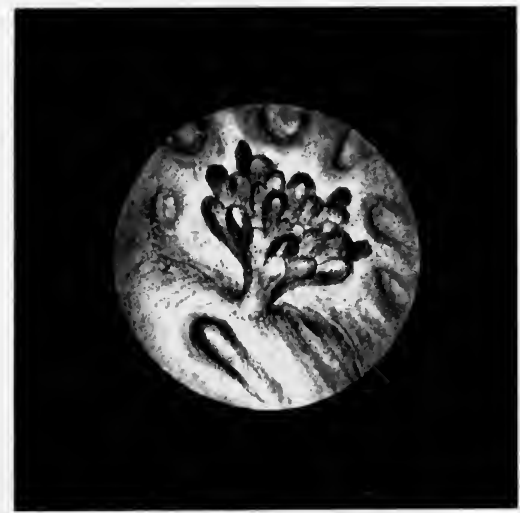

Fig. 53.-Drawing of papilloma of bladder as seen through cystoscope. The tumor springs from the posterior lip of the ureteric orifice.

tumor is declared benign after the most exact examination, the recurrence may be definitely carcinomatous. Biadder tumors may exist for years without giving symptoms. If situated near the internal orifice, the tumor may act as a ball-valve and cause partial retention or "stammering" of the stream. Otherwise the first symptom is frequently hematuria-usually terminal. If the tumor becomes ulcerated and infected, the symptoms of cystitis appear. 
The benign tumors are injurious chiefly because of the infection which they cause, and sometimes because of the constant leakage of blood and consequent anemia. The hemorrhage may be severe enough to fill the bladder with clots.

Malignant tumors are dangerous for the same reasons, and also because of their steady growth. Ureteral orifices may be obstructed, causing renal retention and infection. Metastasis from bladder tumors is relatively late, and early opera- tion offers excellent chances for cure, or at least for a considerable prolongation of life.

The presence of bladder tumor should be suspected in every case of hematuria. In order to detect bladder tumor early, cystoscopy should be done in every case of hematuria, even if the bleeding has stopped, and in every case of unexplained pyuria. The treatment should be based upon the cystoscopic picture. It is poor surgery to open the bladder for tumor unless the nature and position of the growth are known.

Treatment of the benign types consists simply of removal of the tumor. In the case of solid, meaty tumors, such as fibromata, this must be done by open operation. Benign papillomata may be removed by the intravesical route, that is, by thermocoagulation with the high-frequency current.

The spark is applied to the tumor by means of an insulated wire passed through one of the catheter channels of the cystoscope. The unipolar method is generally used in this country. As the tip of the wire lies in contact with the tumor, the cur- 
rent is turned on, and the papilloma rapidly disintegrates at the place where the wire touches it. From one to fifteen or twenty treatments may be required, but if the tumor does not respond fairly soon to this method, it should be judged malignant and removed by open operation.

The question of the practicability of this method of treatment must be left to the cystoscopist.

Papilloma of Bladder Removed by Fulguration.-F. E. H., a traveling salesman of forty-six, came to me $\Lambda$ pril 12,1915 , because of hematuria. Thirty years before he had passed a stone; twenty-five years before he had had "orchitis." Past history otherwise negative.

In October of the preceding year he first noticed a little blood at the end of urination. This lasted for some time, with no discomfort whatever. The blood then disappeared until one week ago. Lately he has noticed that urination may be suddenly interrupted. Frequency every two hours by day, once at night. General examination negative. Urine tinged with blood.

Cystoscopy showed a typical papilloma about as large as a cherry springing from the outer side of the right ureter and hiding the orifice. The bladder showed slight trabeculation, but was otherwise normal. The tumor was fulgurated nine times between May 1st and July 2d. On the latter date a small stub remained, which was thoroughly fulgurated. He was cystoscoped at intervals of six months. In August, 1916, one tiny bud was seen, just behind the right ureter. This bud was fulgurated twice. He was last cystoscoped December 28, 1917, at which time there was no sign whatever of any tumor.

Papilloma of Bladder Removed by Open Operation.-S. D. P., a tailor of fifty-five, came to me in March, 1915, with a history almost identical with that of the preceding case. Seven months previously he had noticed a little blood at the end of urination. He was given formin tablets, and the bleeding stopped. Three weeks ago it began again. He urinates every two hours by day, twice at night. No loss of weight. General examination negative. Urine hazy, with specks of blood floating in it. Cystoscopy showed a papillary tumor about $1 \frac{1}{2}$ inches in length arising from the left lateral wall of the bladder, about 1 inch outside the left ureter. Owing to the mental attitude of the patient operation rather than fulguration was advised. On March 10, 1915, suprapubic cys- 
totomy, extraperitoneal, was done. A tumor with small pedicle was removed with the underlying mucosa. The bladder was closed tight and a rubber tissue drain placed down to the bladder wall. Primary healing

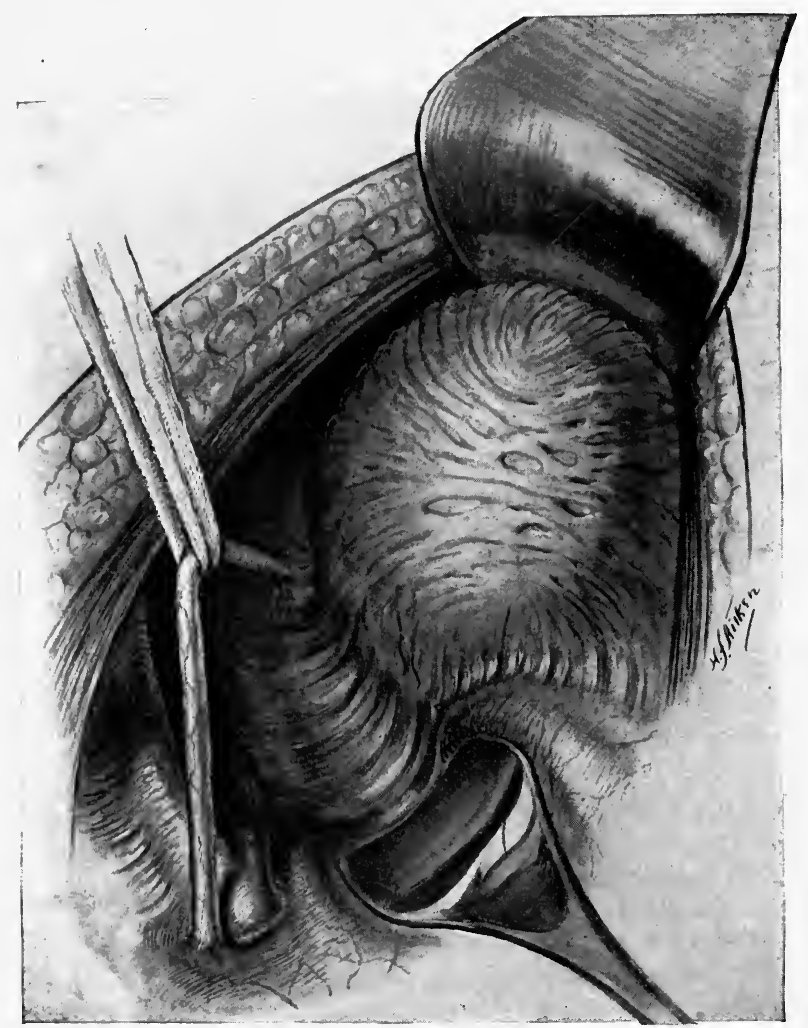

Fig. 54.-Implantation of ureter. Peritoneum stripped off bladder. Ureter held up by tape, preparatory to being cut and reimplanted.

took place. The tumor, on section, turned out to be a simple papilloma. There was no evidence of infiltration of the base.

The patient was cystoscoped every six months up to June, 1917. As he then showed no sign of recurrence, he was told to report in one year. 
Malignant tumors must be removed; not only the tumor, but that portion of the bladder wall upon which they are borne, must be excised. Partial cystectomy is possible in a
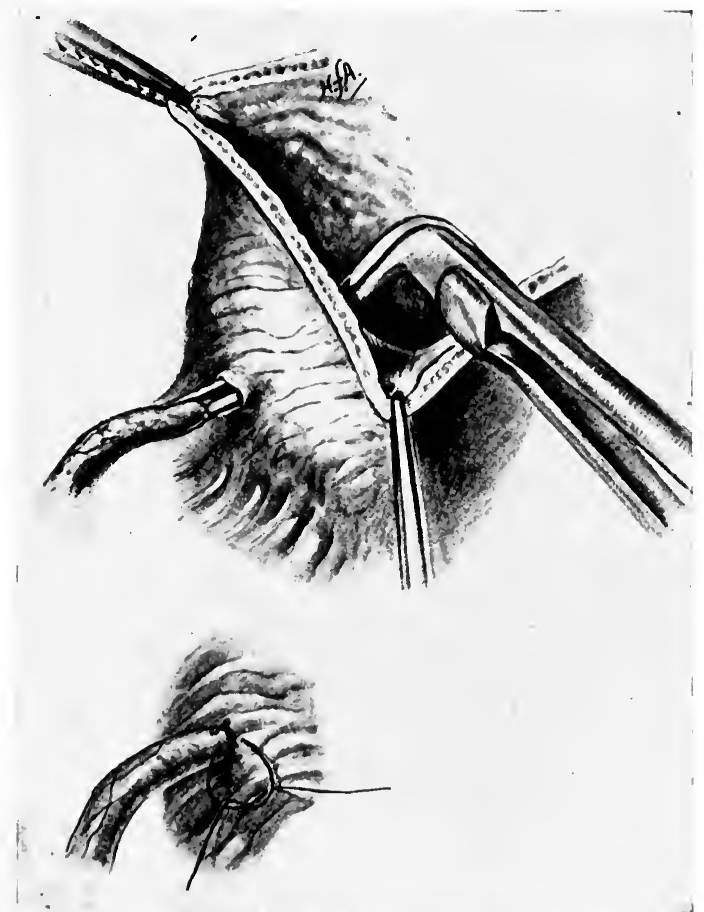

.4

Fig. 55.-Implantation of ureter. Cut end of ureter being drawn through bladder wall. Ureter fastened at point where it enters bladder wall by two sutures of chromic catgut.

great many of these tumors, even if a ureteral orifice is involved. In that case the ureter is transplanted into another part of the bladder. If the growth is too widely spread about the bladder to permit of this, radical treatment means drain- 


\section{IO AN OUTLINE OF GENITO-URINARY SURGERY}

age of the kidneys either by ureterostomy, by implantation of ureters into the bowel, or by nephrostomy, and total removal of the bladder. The operation of cystectomy, followed by the establishment of urinary fistulæ on to the surface of the body, is not to be recommended lightly. Yet in the case of certain bladder growths, which, although wide-spread

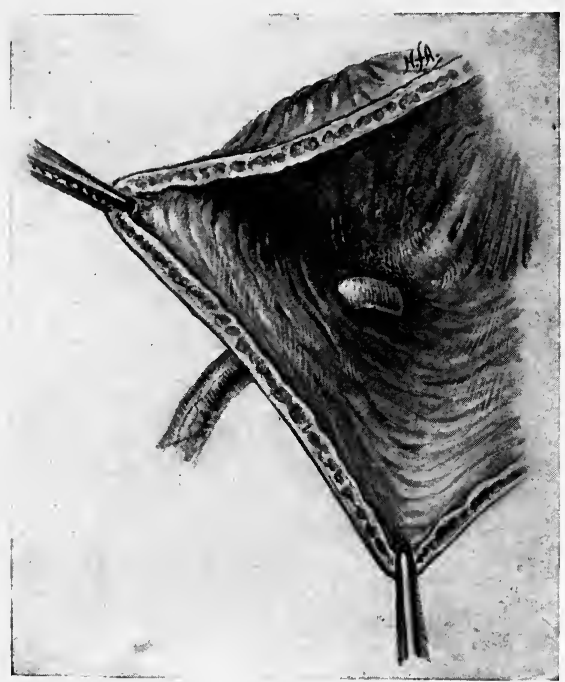

Fig. 56.-Implantation of ureter completed. Ureter projects $\frac{1}{2}$ inch into the bladder.

locally, have not produced detectable metastases, the operation offers far more to the patient than does the palliative cystostomy or simple vesical curetage and cauterization, which is generally done.

In considering the pros and cons of cystectomy one should remember that unless a man has the leisure and the intelligence to care for his nephrostomy tubes or his ureterostomy 
apparatus he had better not have either of those operations done even to save his life.

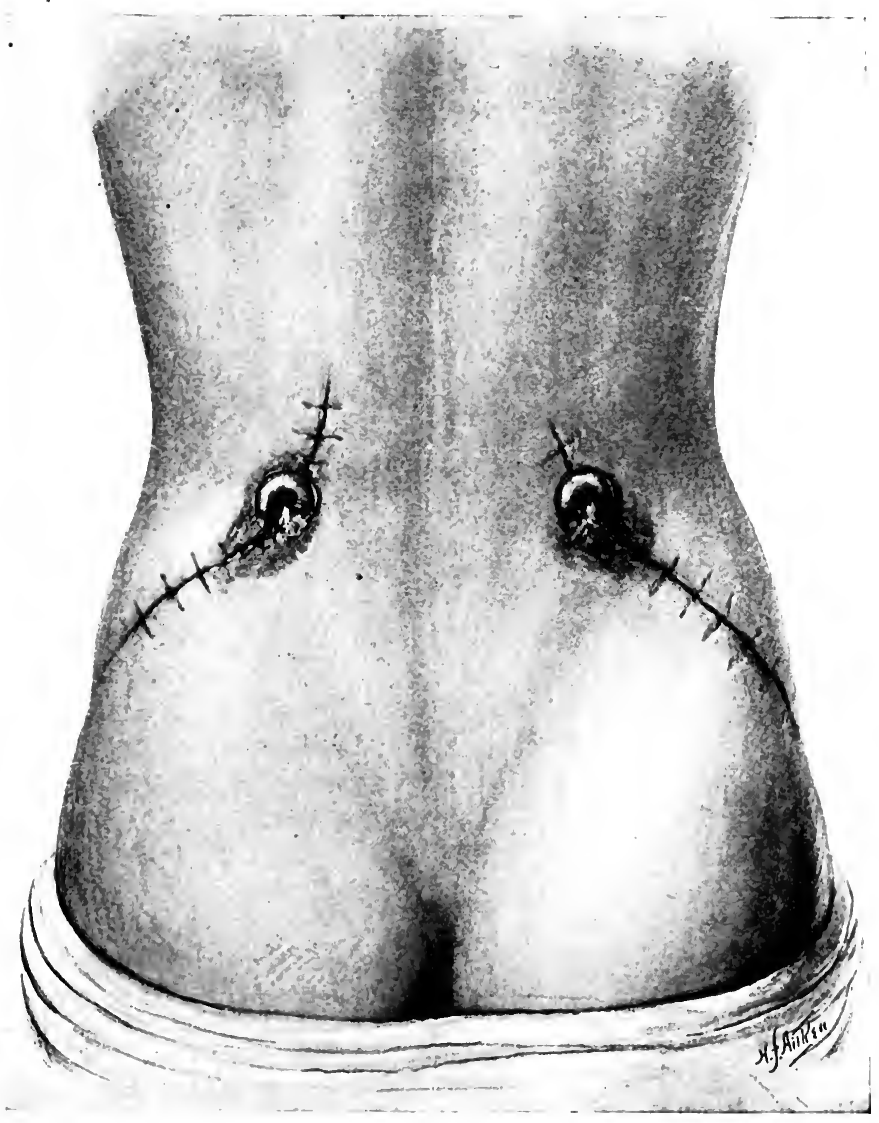

Fig. 57.-Situation of the fistula in bilateral nephrostomy. The tubes which drain the renal pelves have been removed.

If none of the above measures can be employed, simply opening and draining the bladder, with cauterization of the 


\section{I 2 AN OUTLINE OF GENITO-URINARY SURGERY}

tumor, will make his remaining days easier and perhaps a little longer.

Within the last year or two rather encouraging reports have come from Hugh Young and from Barringer in regard to the value of radium in the treatment of bladder carcinoma: As yet this treatment is not on an established basis. With some tumors, even inoperable ones, the use of radium gives wonderful results. Other tumors do not improve permanently at all. Unquestionably this treatment has great possibilities; at present the consensus of opinion, even among its advocates, seems to be that if a tumor is operable it should be removed. Radium should be reserved for those tumors which cannot be removed with any hope of cure, or for recurrences after operation.

The choice of treatment demands careful consideration and a thorough knowledge of the patient-of his renal condition, his general health, his economic condition, and, indeed, of his mental attitude toward life. A radical procedure such as cystectomy should not be attempted without the understanding and co-operation of the patient.

Vesical Calculus.-Stone in the bladder may occur at any age and in either sex. The condition is most frequently found in bladders in which a residual urine exists. The stagnation of the urine and the alkalinitywhich results from bacterial growth are both factors in deposition of urinary salts. A few cases of aseptic stone are seen in which the symptoms are entirely due to the mechanical effect of the foreign body. Pain when be- 
ing jolted or jarred is fairly pathognomonic, especially if accompanied by fresh blood in the urine.

In prostatics, pain in the bladder and vesical irritability which do not yield readily to hygiene and balsamics are very suggestive of stone. The possibility of vesical calculus should also be borne in mind in cases of urinary incontinence in childhood. The fact that the urine is uninfected does not necessarily exclude stone.

Ordinarily, unless the urethra is obstructed, a stone can be detected by filling the bladder with fluid (boric acid), passing a small sound (No. 20 French), and gently moving the beak about the bladder. It should be remembered that encrusted tumors may give the same grating sensation. The diagnosis is best made by the cystoscope, although the $x$-ray will tell a good deal about the size and density of the calculus. The treatment consists in crushing and removing the stone by the operation of litholapaxy, which is far superior to cystotomy, in that the patient is confined to bed for a few days only, and is not subjected to the risks of wound sepsis and a urinary fistula. There are some cases in which the open operation is preferable, in fact, necessary. Extreme youth is a contraindication to litholapaxy. Calculi complicating diverticulum or obstructing prostate may be very difficult to crush, and may have to be removed by cystotomy.

Rupture of the bladder occurs most frequently in accidents in which the victim is crushed between two heavy bodies or is run over by a very heavy vehicle. It may happen to women during childbirth if the bladder is not emptied before- 


\section{I4 AN OUTLINE OF GENITO-URINARY SURGERY}

hand. The rupture usually breaks into the loose perivesical tissue; less often, into the peritoneum itself. The symptoms are severe shock, the passage of bloody urine by urethra, or inability to void. If the rupture is intraperitoneal, the abdomen becomes distended and free fluid may be demonstrated. If it is extraperitoneal, the escaped urine infiltrates the areolar tissue of the pelvis and later forms an abscess. If, following an injury, the patient passes only a little blood and no urine, the diagnosis of rupture of the bladder is probable. Rupture of the urethra must be ruled out by the passage of a catheter. Through this the surgeon injects 4 ounces of boric acid solution into the bladder. The return flow should be accurately measured. If it is less than the amount injected, exploration should be done. If all the fluid returns, but is bloody, one may reason that the rupture is incomplete, and is certainly not intraperitoneal. It is justifiable to fasten a large soft-rubber catheter in the urethra and keep the patient under close observation. Catheterization should not be done unless one is prepared to operate immediately in case a definite rupture exists, for with catheterization infection sets in, and converts a comparatively harmless extravasation into a culture-medium of the best sort. If at operation rupture is found, it should be sutured, if accessible; the bladder should be drained with a large rubber tube, and the extraperitoneal space also drained. If in doubt whether or not to operate, one should elect the more radical course, as rapid exploration is less harmful than an undrained extravasation. 
Barney, J. D.: The Care and Management of the Tabetic Bladder, Boston Med. and Surg. Jour., 1910 and 1911, clxiii, 933-937; clxiii, 965-970; clxiv, 13-17.

Caulk, J. R.: Encrusted Cystitis, Trans. Amer. Assoc. Genito-urin. . Surg., 1914, ix.

Fowler, H. A.: Syphilis of the Bladder, Jour. Amer. Med. Assoc., 1917, lxix, 1399-1402.

Geraghty, J. T.: The Results of Treatment of Bladder Tumors, Jour. Amer. Med. Assoc., 1917, Ixix, 1336-1342.

LANE, C. G.: Bilharziasis. Report of a Case with Appendicitis. literature since 1904, Boston Med. and Surg. Jour., 1910, clxiii, 937-940.

Sмiтн, G. G.: Chronic Cystitis in Women Not a Disease, Jour. Amer. Med. Assoc., 1913, Ixi, 2038-2041. Bladder Disturbances Ine to Nerve Lesions, Jour. Amer. Mled. Assoc., 1917, lxix, 1323-1327. 


\section{CHAPTER XIII}

\section{DISEASES OF KIDNEYS AND URETERS}

DISEASES of the upper urinary tract may be discovered through routine examination of urine, but, as a rule, the patient's attention is attracted by some symptom caused by his condition.

The important signs and symptoms of renal or ureteral disease, more or less in the order of their importance, are:

(1) Urinary abnormalities.

(2) Pain.

(3) Disturbances of urination.

(4) Fever.

(5) Abdominal tumor.

(6) Signs of uremia.

1. Urinary Abnormalities.-The abnormality most startling to the patient is hematuria. Renal hematuria may give the urine a bright color, but, as a rule, is characterized by a "smoky" hue. Hematuria may be the only sign of renal disease. The causes are numerous. Early chronic nephritis may cause quite profuse bleeding. Varix of a renal papilla, early hypernephroma, or renal stone may be the cause; there may be no pain and no tumor-simply a silent bleeding. Hematuria accompanied by pain is significant of stone; accompanied by tumor, it suggests hypernephroma. If no 216 
localizing signs appear, such as pain or tumor, one cannot tell whether the bleeding is from kidney or from bladder, except by the cystoscope. Once again let the rule be emphasized: Every case of hematuria, with the sole exception of those in which the hemorrhage is clearly from the prostate, should be cystoscoped at once.

Hematuria of Chronic Nephritis.-L. L. P., age thirty-eight, veterinary surgeon, seen February 28, 1914. I'neumonia when five years of age; tonsillitis sixteen years ago. Has "grip" once every year. Three years ago had an altack of weakness and dark-colored urine, lasting for one weck. Last winter had a second similar attack. This winter one slight and one severe at tack. Has had headaches for past year, which he thinks are due to constipation. He has never had edema and has no frequency of urination.

Physical examination showed an apparently healthy man. (ieneral physical examination negative. Heart not enlarged: sounds clear. Systolic blood-pressure 180. Neither kidney palpable or tender. Phthalein test, intramuscular: color appeared in seven minutes; 45 per cent. was excreted in the following hour. Urine hazy; acid; sp. gr. 1014; albumin large trace; sugar 0 . Sediment: much blood; some pus: a few casts; no bacteria seen. Culture negative. Guinea-pig inoculation negative.

Cystoscopy March 18, 1914, shows normal bladder. Both ureters catheterized. Grine from right shows blood-cells; a few finely granular casts; no pus or bacteria. The left shows much blood; no pus or casts: motile bacteria. Culture: right, negative; left, staphylococcus.

During the next month the urine gradually cleared up. The specific gravity remained around 1005 and there were constantly a few casts in the sediment. May 15, 1914, says he has felt very well, and that urine has been clear. Urine, however, was found to be hazy; sp. gr. 1016: albumin very slight trace. Sediment: some red blood-cells. Bloodpressure, systolic, 165. Nothing further was heard from him until January 24, 1916, when he had another attack of hematuria. Ile had had none for the previous two years. The urine was very bloody, with rare hyaline cast. Systolic blood-pressure 160. Patient was jaundiced. It now seemed certain that his bleeling was due to chronic nephritis. He was placed under the care of a medical man and was not seen by me again. On May 1, 1917, he died of nephritis. 


\section{I 8 AN OUTLINE OF GENITO-URINARY SURGERY}

Renal Hematuria Due to Focal Nephritis or to Varix of the PapillaSo-called "Essential Hematuria." C. B. G., male, bookkeeper, age thirty-six. Gonorrhea eight years previously. Three years ago had pains in the back, and later passed blood in the úrine. Cystoscopy at this time showed that the blood came from the right kidney. A guinea-

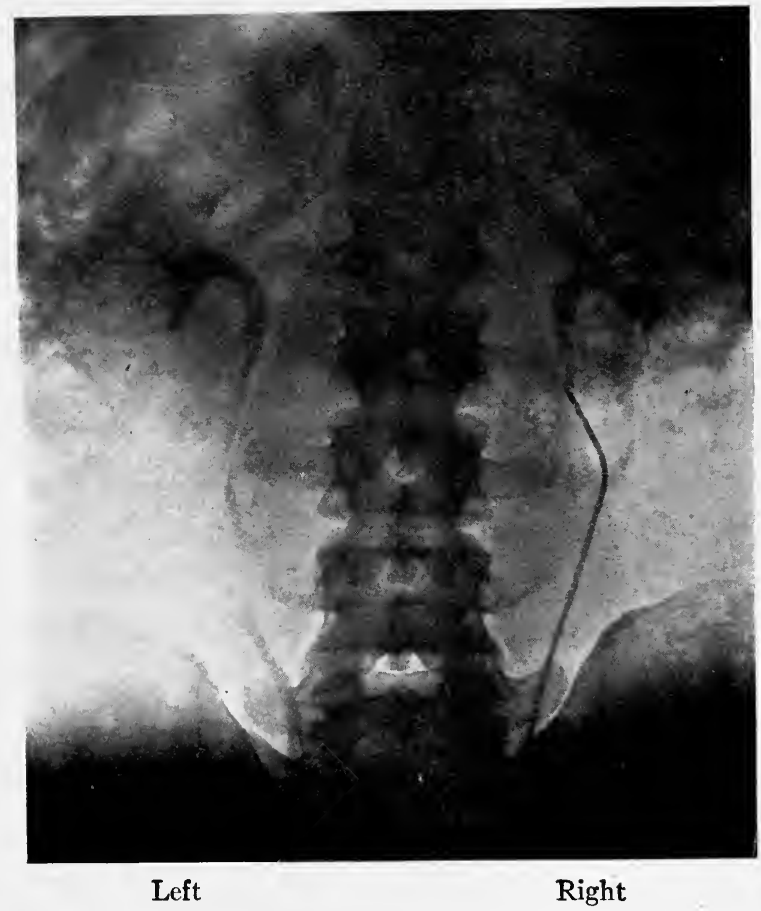

Fig. 58.-Right renal hematuria. No definite abnormality of pelvis of either kidney, although upper calyx of right kidney is somewhat widened. ( $x$-Ray by Dr. L. B. Morrison.)

pig inoculated at the time was negative for tuberculosis. After six weeks the bleeding stopped, not to recur again until one week ago.. $x$-Rays show urinary tract negative. $x$-Ray of chest shows well-healed lesion of glands about root of right lung. November 20,1917, blood-pressure 164 systolic, 112 diastolic. November 22, 1917, systolic 135. Lungs 
and heart normal. Urine clear; sp. gr. 1006; albumin slightest possible trace; sugar 0. Sediment: a few blood-cells; occasional leukocyte.

Cystoscopy December 1, 1917: Right ureter catheterized to kidney. Passage of catheter followed by a gush of bloody urine from ureter. On left side no catheter would pass farther than $5 \mathrm{~cm}$. Both sides were injected with thorium. Pyelograms showed normal pelves. The normal outline of the right kidney pelvis seemed to us to exclude tumor of the kidney as a cause of hematuria (Fig. 58). Hypernephroma which caused bleeding three years before should have by this time caused abnormality of the pelvis. Diagnosis seemed to us to be either early chronic nephritis or bleeding from a varix in one of the renal papilla.

Pyuria practically always means infection. A few leukocytes may occur in aseptic stone or in hypernephroma without denoting infection. With pyuria, the bacteriology of the urine should be investigated, it being especially important to rule out tuberculosis. Colon bacillus or coccal infections, it must be remembered, may complicate stone, tumor, or hydronephrosis.

2. Pain is a symptom of value, which, properly interpreted, often leads to a correct diagnosis. Renal pain is of two kinds - the dull, heavy lumbar ache, which means distention of the capsule (Squier), and is caused by renal retention or by swelling of the renal parenchyma; and the sharp, cutting, intermittent pain, known as "renal or ureteral colic," which denotes a spasmodic hyperactivity on the part of the smooth muscle of the pelvis and ureter. The latter pain radiates along the course of the iliohypogastric and ilio-inguinal nerves-that is, it is referred to the bladder, the labia, the testicle, and the thigh. The dull, constant pain is caused by a beginning hydronephrosis (late hydronephrosis usually does not cause pain) or by acute inflammation of the renal 
cortex. Renal colic is caused by the effort of the pelvis and ureter to express urine past a sudden obstruction, or to express clots, masses of pus, or calculi. The irritation of the mucosa by sharp crystals is sufficient to cause renal colic; in such an event the urine generally contains blood, which points. to an actual wounding of the mucosa.

3. Disturbances of urination may be the only symptoms of disease of the upper urinary tract. The occurrence of frequency as the only symptom of renal stone has been demonstrated. Renal infection, especially tuberculosis, habitually makes itself first felt through disturbance of bladder function. Of course the urinary examination will show the presence of infection in nearly every case. I have seen a pyonephrosis with occluded ureter which gave no signs in the urine nor pain in the kidney, but was the only apparent cause of bladder irritability.

4. Fever is simply another sign of infection, and the occurrence of fever and pyuria together with renal pain or renal tenderness means renal infection. The infection may be simple or may be a complication of another lesion.

5. Abdominal Tumor-A painless abdominal tumor may be discovered by the patient himself or by his physician during routine physical examination. The first obstacle in the way of making a diagnosis may be the difficulty of knowing whether the tumor is of renal origin or not. Abnormality of the urine, such as hematuria or pyuria, casts strong suspicion upon the kidney, but if the urine is normal the question can be decided only by cystoscopic examination. In obscure 
cases pyelography is of very great assistance, as it shows the situation of the kidney with regard to the tumor, and the presence of abnormalities in the shape of the kidney pelvis. The same procedure which tells whether or not the tumor is renal will give the diagnosis if the tumor is of the kidney.

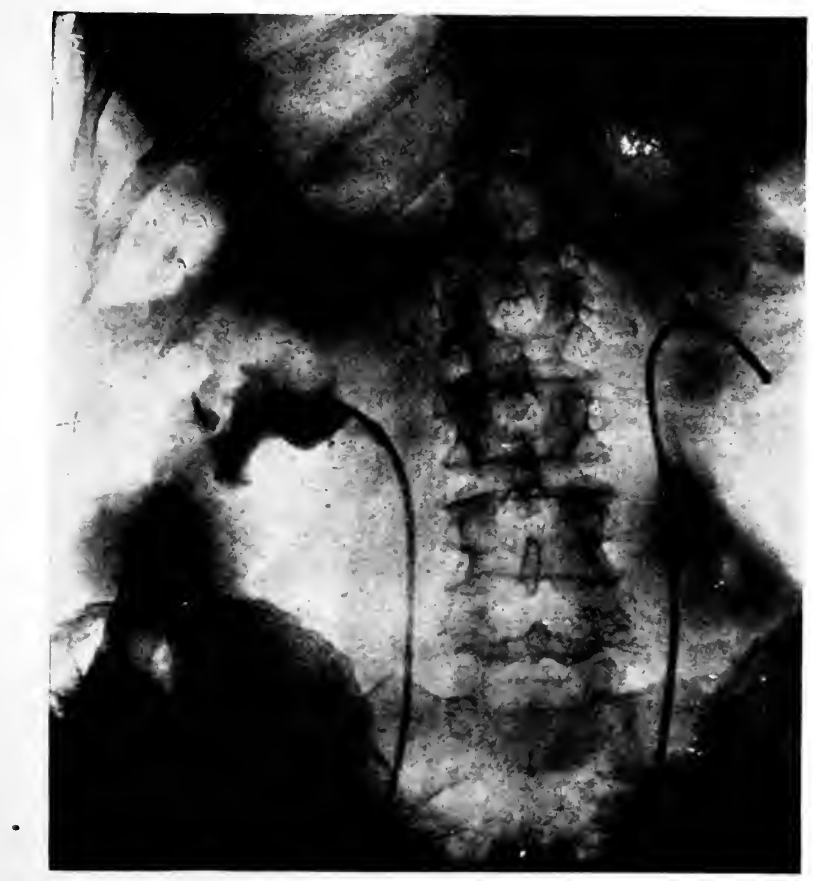

Fiv. 59.-Pyelogram made with thorium. The outline of the upper half of the renal pelvis is distinctly abnormal and suggests the presence of a tumor. ( $x$-Ray by Dr. L. B. Morrison.)

Hypernephroma Diagnosed by Pyelography.-Miss H., a well-preserved woman of serenty-two, whose past history was unimportant, had noticed for about four months that there were times when the urine contained blood. The attacks of hematuria would last for several hours, 


\section{AN OUTLINE OF GENITO-URINARY SURGERY}

sometimes as long as all day, and would disappear as suddenly as they arrived. She had lost some 15 pounds in weight.

On examination, the left kidney was easily palpable and felt somewhat enlarged. The urine sometimes contained blood, at other times was normal. I was asked by Dr. E. H. Risley: whose patient she was, to investigate her renal condition.

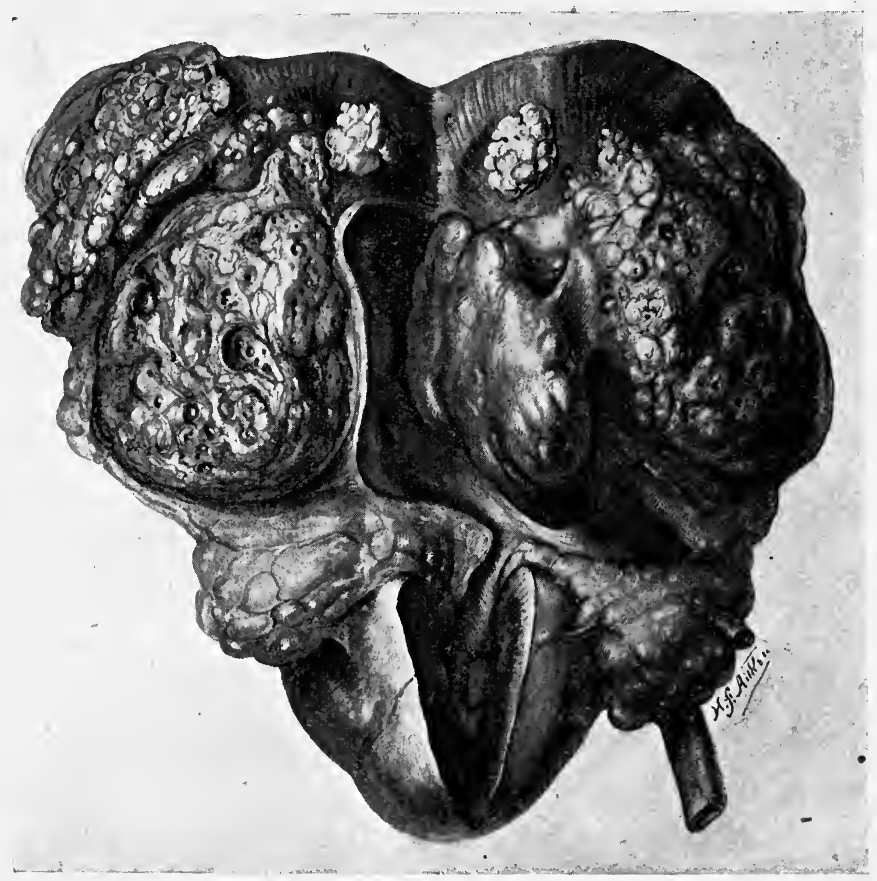

Fig. 60.-Hypernephroma. A pyelogram of this kidney is shown in Fig. 59. Note the mass of tumor tissue obliterating the upper part of the pelvic cavity, as is suggested by the pyelogram.

Cystoscopy, December, 1917: The bladder was normal. Divided functional test showed prompt output of phthalein from both sides; the amount excreted appeared about equal. A pyelogram showed definite deformity of the upper part of the renal pelvịs (Fig. 59), because of which the diagnosis of hypernephroma was made. 
Dr. Risley removed a somewhat enlarged, knobby kidney (Fig. 60). When split open, the upper half was seen to be composed of tumor tissue. A tongue of new growth projected into the upper part of the pelvis, and was the cause of the deformity shown in the pyelogram. Microscopic examination showed the tumor to be hypernephroma. The patient made an uneventful convalescence.

Without cystoscopic examination it is at times impossible to say whether a tumor unaccompanied by urinary abnormalities is of the kidney. The situation of such a tumor beneath the ribs, and the fulness in the costovertebral region which is evident upon bimanual palpation, suggests a renal origin, but cysts of the liver upon the right and tumors of the spleen upon the left may be indistinguishable from renal tumors by ordinary methods of examination.

Differential Diagnosis of Abdominal Tumor.-M. W. S., age twentysix, electrician. Case of Dr. Hugh Williams at the Massachusetts General Hospital. No. 210,403. September 8, 1916. Always well and strong. Six years ago was sick for about three weeks with jatundice. Three months ago noticed a lump in the left side of the abdomen just below the ribs; it has grown no larger since then, and has caused no pain. Physical examination negative save for smooth, non-tender, rounded tumor, which protrudes from under left costal margin and extends downward as far as the umbilicus. No notch could be felt. The mass seemed definitely fluctuant. Urine was normal. Leukocytosis 28,000 and 15,000. Polynuclears 84. Lymphocytes 16. Red cells normal. Surgical and medical consultants expressed their belief that the tumor was due to (1) polycystic kidney, (2) cystic kilney. Cystoscopy: Bladder normal. Urine from left kidney showed a few red blood-cells and leukocytes. Radiogram (Fig. 61) showed the ureter catheter curving across spine to right side, and back again to left side. Renal pelvis was low. Genito-urinary consultation: "Cystic tumor pushing kidney down." Total function: One hour, 45 per cent. Echinococcus complementfixation test and Wassermann negative.

Operation was done by Ir. Hugh Williams. A cystic spleen was removed, which the pathologist reported as dermoid in type. Uneventful recovery. 
The feeling of fluctuation in such tumors is not a safe guide to their character. Soft, congested solid tumors, such as some hypernephromata, may feel less solid than do some cystic tumors tense with fluid.

The presence of blood in the urine combined with a tumor in the renal area is so suggestive of hypernephroma that

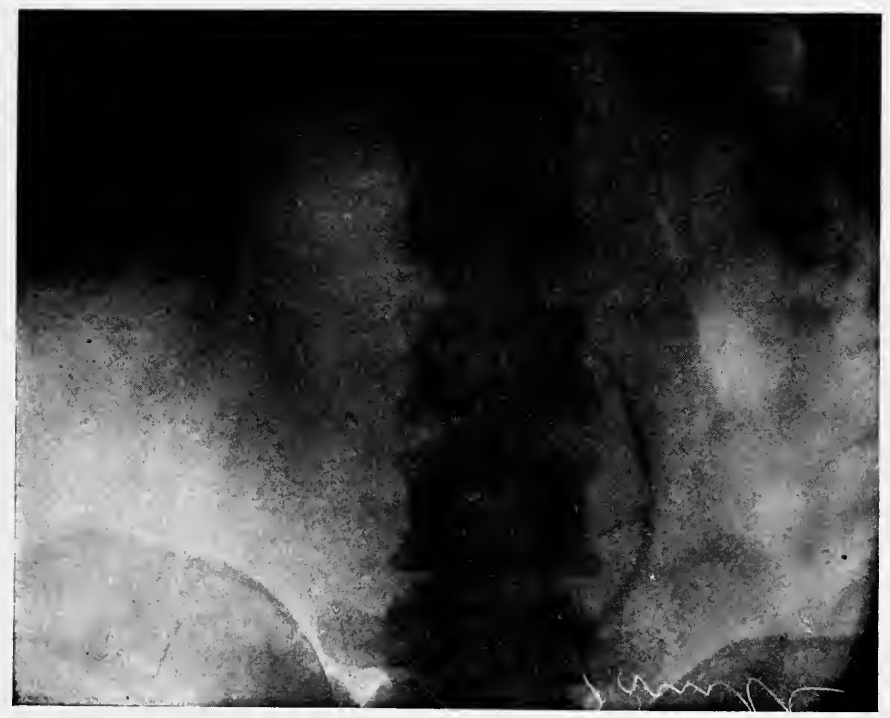

Left

Right

Fig. 61.--Radiographic catheter in ureter. Kidney pushed down by tumor of spleen (Mass. General Hospital).

operation should be thought of at once. Cystoscopy should be done to ascertain from which side the blood is coming, as otherwise one cannot be sure that the hemorrhage is not caused by bladder tumor, stone in the other kidney, or some other cause not connected with the tumor in the flank. The 
finding in the urine of a slightest possible trace of albumin and a low specific gravity, especially if these are accompanied by an increased blood-pressure, suggest that the tumor may be a polycystic kidney. In this case the tumor may be bilateral, with palpable nodularities of the surface.

Variability in the size of the tumor suggests hydronephrosis, and this suspicion is upheld by a definite history of the passing of large quantities of urine coincident with the diminution of the tumor. It is to be remembered that such a kidney occasionally bleeds following sudden evacuation of its contents.

6. The symptom-complex which at first sight seems least likely to be due to kidney disturbance, but which, in reality, is frequently the sign that awakens the patient to a knowledge that something is wrong, is the symptom-complex of renal insufficiency. This manifests itself through the patient's general condition and particularly through his digestion. Afflicted by headache, a sense of malaise, flatulence, loss of appetite, and nausea, the patient determines to see his doctor and get a "tonic." If his physician does a general physical examination and examines the urine he will probably be able to render a real service.

Renal symptoms of this character are most frequently secondary to obstruction in the lower urinary tract. Tabetics, prostatics, individuals with diverticula which press upon the ureters, may not realize they are sick until the signs of diminished kidney activity appear. Rectal examination will reveal the presence of any important amount of residual 
urine, and the detection by abdominal percussion of vesical enlargement leads one to the investigation of that portion of the urinary tract.

Bilateral polycystic disease must be borne in mind, and, of course, chronic nephritis of non-surgical types must be excluded. Extensive destruction of renal tissue by stone or infection may develop so insidiously that the victim is unaware of his condition until the signs of uremia appear.

"Essential hematuria" is one of those vague terms used to describe a condition which we do not thoroughly understand. Hematuria not due to stone, tumor, or infection occurs not infrequently. Two explanations have been advanced. Hurry Fenwick believes that many of these cases are due to varicosities upon the papillæ. Others hold that the bleeding is due to areas of sclerosis in the kidney-the so-called Weigert kidney. The hemorrhage is painless, intermittent, and at times profuse. It occurs usually in persons who are in the second half of life, and may be severe enough to cause serious anemia. Even if this is not the case, every renal hematuria must be studied by function test and pyelography or else subjected to an exploratory operation. The danger of overlooking an early hypernephroma is too great to permit of "watchful waiting." An absolutely normal pelvic outline, with no enlargement of the kidney, may justify a diagnosis of "essential hematuria," without actual exploration of the kidney, but, especially if the hemorrhage is at all profuse, operation is the safest procedure. The kidney need only be delivered and palpated; nephrotomy is unnecessary. 
If the bleeding should prove to be that of "essential hematuria," and not due to tumor, the operation is not in vain, for the bleeding of essential hematuria is practically always cured by decapsulation of the kidney.

Renal Hematuria Cured by Decapsulation.-P. J. I:, age forty, single. Admitted to the Massachusetts General Hospital July 1, 1915, with a diagnosis of "essential hematuria," probably due to varix.

The past history was negative. His present trouble began about two and a half months ago, when he fell from the arm of a chair, striking his left side against a step. On the following day he noticed that he was passing bloody urine, and this has continued up to the present time. Urine more bloody in the morning than toward the end of the day; on several occasions he passed clear urine in the afternoon. Has never had any frequency, pain, or burning sensation, and feels perfectly well.

Physical examination showed a well-developed and nourished man. General physical examination negative. Urine: Bloody; acid; specific gravity 1020; albumin trace; sugar 0 . Sediment: Many red and white blood-cells. Cultures negatire. $x$-Ray, pelvis injecterl with 2 c.c. of argentide, showed a low right kidney; pelvis not completely filled. July 3d, cystoscopy: Left catheter passed easily to pel:is; right passed easily to within 2 inches of the kidney, where it stuck; 1 c.c. phenolsulphonephthalein injected into vein. Appearance time on left four and a half minutes, on right seven minutes; 20 per cent. on left, 6 per cent. on right, excreted in fifteen minutes. No leakage into bladder. Preoperative diagnosis: Tumor of kidney, with obstruction of pelvic outlet.

July 3, 1915, operation: 5-inch oblique kidney incision. A normally placed kidney freed and delivered. There were unusually dense adhesions between lower pole and ureter. These were thoroughly freed. Careful palpation of kidney showed no suggestion of tumor or stone. Capsule stripped. Fixation not done, as kidney fell of its own weight into a better position than could be obtained by fixation. Rubber tissue drain.

Convalescence uneventful, and on July 15 th the patient was discharged relieved. February 18, 1918, the patient reported for examination. He had had no hematuria since operation. Urine was clear, no albumin. Blood-pressure was normal and the kidney was not palpable.

Summary.-Unilateral renal hematuria of two and a half months' duration cured by decapsulation and freeing of reno-ureteral adhesions. Diagnosis is a little in doubt. Radiograph suggested pelvic retention, 
but bleeding may have been due to a varix of the papilla. The findings at operation do not explain decreased renal function from diseased kidney.

Decapsulation in Nephritis.-Decapsulation of the kidney as a means of arresting the progress of nephritis was performed by Edebohls in 1898. He believed that the increased and adequately maintained blood-supply to the kidney established by the operation leads to gradual absorption of the interstitial or intertubular inflammatory products and exudate, thus permitting the re-establishment of a normal circulation. Of 102 patients operated upon by him, 33 were cured and 11 improved. Experimental and pathologic evidence has, as a rule, not sustained Edebohl's contention. Despite this fact, as careful an observer as John Lovett Morse, basing his opinion upon a number of carefully followed cases of nephritis in children upon whom Edebohl's operation had been done, comes to the conclusion that "no child ill with acute nephritis should be allowed to die without giving it the advantage of the chance offered by this operation. It may prolong life for considerable periods in a not inconsiderable number of cases of chronic nephritis, and very possibly, in rare instances, result in cure. It should, therefore, always be considered in all cases of chronic nephritis in childhood which are not responding reasonably well under medical treatment."

Nephroptosis, or movable kidney, is a very common condition, especially in women. The right kidney is more liable to be affected than the left. Abnormal mobility sometimes follows a rapid loss of weight, and in that case is caused by 
the absorption of fat from around the kidney. At other times it is the result of faulty posture, and is due to (1) general ptosis of all the abdominal organs, (2) the straightening out of the lumbar curve, and consequent obliteration of thr shelf upon which the kidney ordinarily rests.

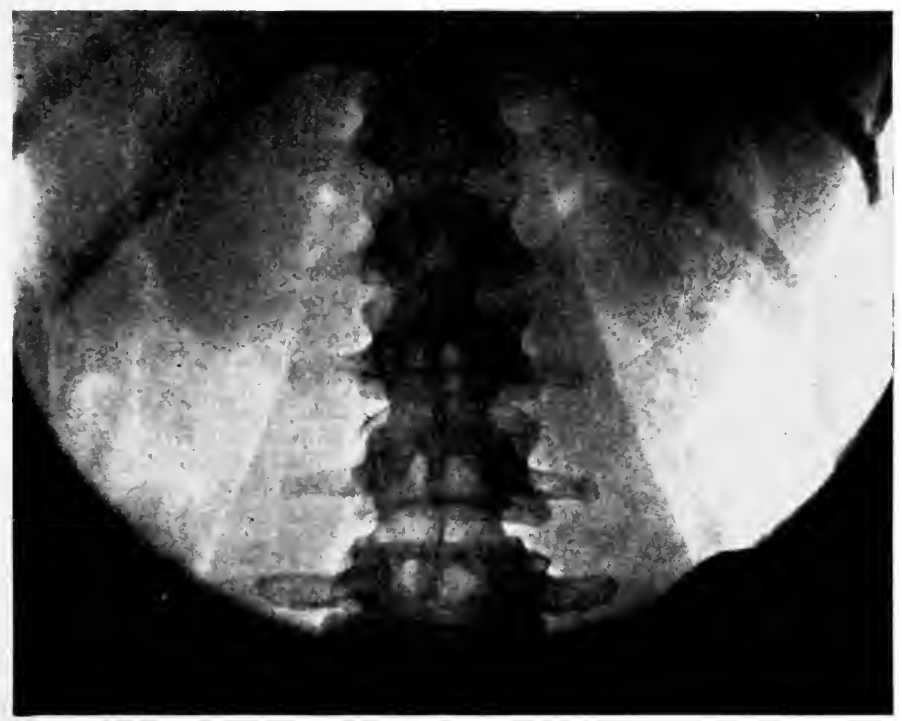

Fig. 62.-Showing normal position and outlines of kidneys. Patient standing (Mass. General Hospital).

It is not at all unusual to be able to palpate the lower part of the kidneys in women; mobility of that degree can hardly be called abnormal. When the kidney descends below the costal margin and can be palpated in its entirety, it may be said to be abnormally mobile. Even such mobility, however, does not usually cause symptoms or do any particular harm. 


\section{0 \\ AN OUTLINE OF GENITO-URINARY SURGERY்}

The ureter, like the intestine, can propel its contents in spite of curves and kinks, unless at some one of the kinks it is adherent. When the ureter kinks over a band of adhesions or an aberrant vessel, the free passage of urine is, of course, obstructed, and renal retention, with its various symptoms, results.

Movable kidneys sometimes cause a dull ache, presumably from the drag upon the renal mesentery or from the congestion caused by kinking of the renal vein. The typical pain, however, is excruciatingly sharp, the pain of sudden distention of a renal pelvis which cannot empty itself, and is known as "Dietl's crisis." If attacks of renal pain can be relieved by pressing the kidney upward into position or by a change of posture, this fact is of much diagnostic value.

There are many cases of pain, apparently of renal origin, associated with normal urine and negative $x$-ray findings. Most of them are never explained. To attach the blame to a condition of mobility demands more than the mere fact that the kidney descends somewhat on inspiration. There must be evidence of pelvic dilatation in a pyelogram, there must be reproduction of the pain when the renal pelvis is distended, and there must be ruled out such possible causes of pain as gall-stones, spinal lesions, occupational strains, and so forth.

The operation for the relief of renal mobility is nephropexy. The capsule is stripped and its four corners are sutured to surrounding muscles, thus suspending the kidney in a sling. If done in properly selected cases the operation gives great relief. If done in the face of general ptosis or faulty posture 
it will be followed by a return of the kidney to its old habits. Some years ago many kidneys were operated upon for mobility. Today, with better diagnostic measures at hand, we do the operation less frequently.

Movable Kidney-Nephropexy.-IV. P. F, age forty-four, single. Admitted to the Massachusetts ('eneral Iospital September 8, 1916. Diagnosis: Nephroptosis.

Past IIistory.-Chancre twenty-two years ago; rash two months later; treatment for three years. (ionorrhea four years ago; discharge for three months. Occasionally since then there has been slight urethral discharge.

Present Illness.-Twelve months after patient became infected with gonorrhea he had a sense of pressure in the region of the right kidney. Since then this sense of pressure, rather a dragging down feeling, has bothered him constantly. There has been no severe pain, no nausea or vomiting. No stones or gravel passed with urine; no hematuria, dysuria, or frequency. Pain comes without strain or exertion and is not affected by motion; always present when patient is standing, disappears on lying down.

Physical examination showed a well-dereloped and nourished man. Abdomen full, tympanitic throughout, except in upper right quadrant, where there is dulness. Right kidney felt $10 \mathrm{~cm}$. below costal margin when patient is upright, $2 \mathrm{~cm}$. below when reclining. Edge is smooth, about normal in size and shape, tender on pressure. Urine: Clear, acid; no albumin; no sugar; rare pus-cell in sediment. Renal function: . Ippearance time eight minutes; first hour 30 per cent.; second hour 20 per cent. Cystoscopy: Moderate amount of pain and intolerance. Mucosa normal, ureters normal, but orifices are at such an angle that catheter was passed with considerable difficulty; 9 c.c. of methylene-blue injected into right pelvis before there was a return flow. Argentide ( 4 c.c.) injected for $x$-ray, causing severe renal colic. $x$-Ray: Outlines of kidney are distinctly seen, appearing normal in size and shape. Pelvis' of kidney not sufficiently filled to show outlines.

Operation (September 9, 1916).- Vertical lumbar incision on right side carried forward at lower end. Very thick musculature made delivery of kidney difficult. A movable kidney of normal size was freed. Several cysts on anterior surface punctured. No stone felt. Kidney capsule split longitudinally and stripped; four corners tied with Pagenstecher and sutured to adjacent musculature. Rubber tissue drain. 


\section{2 \\ AN OUTLINE OF GENITO-URINARY SURGERY}

Convalescence uneventful. Patient discharged relieved September 24, 1916.

Summary.-A case of very movable kidney in an unusually muscular man who had no general ptosis. Nephropexy done. No reason for mobility found.

Renal Infections.-Aside from tuberculous infections of the kidney, which will be considered in Chapter XIII, the great majority of renal infections ( 70 or 80 per cent.) are due to the colon bacillus. Staphylococcus and streptococcus infections are next in frequency, and then come the rarer infections by the Bacillus mucosus capsulatus, gonococcus, and Bacillus pyocyaneus.

The route by which infection takes place has been the subject of conflicting opinions. Definite evidence has been presented, proving that in some cases at least colon infections of the kidney occur through the medium of the blood-stream (Cabot and Crabtree). Other observers have presented experimental evidence that infection may ascend by the lymphatics of the ureter. The latter theory explains certain sequences found in clinical conditions, such as renal infections following infection of vesicles on the same side, better than does the former. The evidence for the theory of the hematogenous route is so incontrovertible that it also must be accepted. The truth probably is that both routes exist, and that sometimes one, sometimes the other, is employed by the bacteria which invade the kidney.

Infection ascending by means of the column of fluid contained in the lumen of the ureter is impossible except in cases of urethral obstruction, in which, as a result of back pressure, 
the ureterovesical valves become incompetent and the entire upper urinary tract becomes distended with stagnant, infected urine.

In experimental animals a traumatized kidney is more susceptible to infection, and this undoubtedly holds true in man. In man, trauma of the kidney is caused by stone or by distention with urine, such as occurs through blocking of the ureter or through obstruction at or below the bladder neck. When the distention is suddenly relieved, as by complete catheterization of an overdistended bladder, a congestion of the kidney follows, which reduces the resistance of that organ to infection. Autopsy on cases of prostatic obstruction who have died following operation show these lesions. In a case of this sort, a man who died two weeks after his operation, the pathologist reported as follows: "Combined weight of kidneys 230 grams. Capsules strip, leaving surface which shows many irregular areas of depression with smaller and larger mound-like masses of kidney tissue resting between them. In many places the surfaces are dotted over with minute to small dirty yellowish areas which yield in instances frank pus. The section surfaces of the cortex show here and there small, pale yellowish areas and slender streaks, some of which yield pus. The pelves are considerably dilated and contain much thin, cloudy, purulent fluid material. The mucosa is reddened and coated in places with dirty, yellowish, shaggy, fibrinopurulent material. Ureters considerably dilated. The mucosa shows in places areas of reddening." The ability of the normal kidney to filter out bacteria with- 


\section{AN OUTLINE OF GENITO-URINARY SURGERY}

out harm to itself has been demonstrated a number of times. If the kidney is damaged, as occurs when a calculus rubs off the superficial layers of the pelvic mucosa, these wandering bacteria secure a foothold.

It is possible that every acute infection of the kidney accompanied by fever and pain involves not only the pelvic mucosa, but ascends by way of the lymphatics into the connective tissue of the kidney between the tubules (pyelonephritis). In certain cases the infection undoubtedly starts in the parenchyma, and later involves the pelvis. Colon infections involving the renal parenchyma almost always quiet down; infections by the cocci are liable to form abscesses or to run so fulminating a course that operative interference is necessary to save the patient from the overwhelming infection (acute hematogenous kidney).

Given, then, an acute infection of the kidney, the prognosis of that particular case is made much simpler if the medical attendant knows what organism is at the bottom of the trouble. If it is the colon bacillus, he may expect the attack to quiet down; if the staphylococcus or streptococcus, he must be prepared for a fulminating infection demanding radical treatment. As the colon bacillus quickly gets into the tubules, pus in the urine occurs early; the coccus infections are more concerned with the intertubular tissues, and pus in the urine is rather a late occurrence. Blood-cells, from the intense congestion, appear early, and the presence in the urine of blood-cells and cocci is of great value in helping make the diagnosis between an acute hematogenous kidney and an acute 
appendix. Blood alone in the urine may accompany an attack of acute appendicitis if the appendix overlies the ureter.

The particular measures to be adopted must be determined at operation. For the less severe cases decapsulation of the kidney and drainage of the pelvis will care for the infection. In the more fulminating type, when the kidney is full of abscesses, nephrectomy is indicated.

In the treatment of acute renal infection in prostatics or tabetics proper drainage of the bladder is imperative. For all cases of renal infection, rest in bed, forced fluids, free catharsis, and bland, light diet are indicated. Hot applications over the affected kidney are soothing.

If the infection is due to the colon bacillus, hexamethylenamin in large doses-15 grains three or four times a day-is very valuable. In coccus infections it is impossible to reach the bacteria in the tissues by any drug excreted through the tubules, so that not much is to be expected from the administration of medicines. The passing of large quantities of water-100 to 200 ounces a day-will be found valuable in quieting the infection, and may be increased by the use of a mild diuretic.

Pyelitis of Pregnancy.-Acute infection of the kidney, manifested by temperature, pyuria, and renal tenderness, may occur at any time after the uterus becomes large enough to press upon the ureters where they cross the pelvic brim. It may come on during the puerperium. Although this process is termed "pyelitis," it is probable that, at least in 


\section{AN OUTLINE OF GENİTO-URINARY SURGERY}

the early period of the infection, there is an invasion of the renal lymphatics as well. The organism is nearly always the colon bacillus; the infection may be really acute or may be an exacerbation of one of long standing. The patient should, of course, be put to bed, given forced fluids and hexamethylenamin, 15 grains, three times a day. Under this treatment the infection will usually subside. Less frequently it will continue and will destroy the kidney. If, therefore, the temperature and pain do not subside within two or three days, more active measures should be instituted. Of these, the first to try is lavage of the renal pelvis by means of the ureteral catheter. The establishment of better drainage through the washing out of inspissated purulent material from pelvis and ureter is probably the real reason for the good results which may follow pelvic lavage.

Pyelitis of Pregnancy Treated by Lavage of the Renal Pelvis.-Mrs. * W. R. T. Age twenty-five. March 17, 1917. Primipara; delivery three weeks ago. After delivery had trouble voiding and was catheterized. Nurse, supposed to do this every eight hours, did not empty bladder completely at any time. It became infected and temperature elevated. Pain in right side. No history of previous urinary infection.

Temperature today $105^{\circ} \mathrm{F}$. Right kidney slightly tender; left not tender. Urine very cloudy, with heavy precipitate of pus; colon bacillus present. Cystoscopy: Base of bladder edematous. After some time a No. 5 ureter catheter was passed up right ureter. Cloudy urine, containing pus and colon bacilli, obtained. Pelvis washed with silver nitrate (1 per cent.). Flakes of pus could be seen issuing about catheter as fluid was injected. Patient had no reaction following cystoscopy. Next evening temperature $103^{\circ} \mathrm{F}$., then fell to normal and remained so.

April 12th: Feels well, but rather weak. Catheter specimen very s'ightly hazy; no albumin. Sediment: Some pus and epithelial cells; a few colon bacilli. Has been on hexamethylenamin, 45 grains a day. Some bladder irritation. Culture shows no growth. Patient has pain 
as bladder is emptied, but no frequency. Hexamethylenamin reduced to $7 \frac{1}{2}$ grains three times a day.

April 25th: Urine clear and clean. No growth. Sediment: Very few red blood-cells. (On April 27 th the urine was clear and clean, with no albumin. Sediment negative. Kidney not palpable.

August 17, 1917: Patient had a chill and an attack of pain in the right costovertebral angle yesterday. Today feels perfectly well. Examination revealed no tenderness. Urine clear and clean; no albumin. Sediment negative.

If two or three irrigations of the renal pelvis fail to allay the process, one must consider either emptying the uterus or draining the kidney. Nephrotomy can be done without necessarily bringing on labor, and is sure to relieve the situation. Emptying the uterus will almost certainly relieve the kidney, but the possibility that it may not do so, and may therefore be an unfruitful sacrifice, favors the direct attack upon the kidney.

Pyelitis of Childhood.--Another rather distinct form of pyelitis is that which occurs in children, especially in girls. The child may run a temperature of $103^{\circ}$ or $104^{\circ} \mathrm{F}$., accompanied perhaps by chills. Many cases, however, have only a very slight rise of temperature. There may be no localizing symptoms whatever, or there may be tenderness in the flank and lumbar region. The diagnosis depends upon the finding of pus or bacteria in the urine. Pyuria may not develop at first. Bacteriuria may be the only abnormality.

The prognosis as to life is good; spontaneous cure occurs in many acute cases. In others the infection persists for years as a low-grade colon bacillus pyelonephritis punctuated by exacerbations, which slowly destroys the kidney. The 


\section{AN OUTLINE OF GENITO-URINARY SURGERY}

pelvis becomes dilated, the cortex thinned. This process may be without symptoms until some new condition, such as pregnancy, supervenes. Then the old infection flares up, and investigation shows a practically useless kidney, a mere shrunken shell of scar tissue in which the colon bacilli are firmly established.

The treatment of acute pyelitis in children consists in confinement to bed, free catharsis, much water, and hexamethylenamin. This drug may be given in 10-grain doses every four hours, six doses being given during twenty-four hours. Many writers believe the best treatment consists in keeping the urine alkaline, and do not use hexamethylenamin at all.

Others have had good results from alternating the reaction of the urine. High vegetable and fruit diet tends to produce alkaline urine, high protein diet (meat and eggs) keeps it acid. Potassium citrate or sodium bicarbonate is given for a few days, then omitted for a few days. Whatever the medication, the essential points in the treatment are rest in bed and the washing out of the kidney pelves by the ingestion of much fluid.

After the attack has cleared up, the patient should be kept on hexamethylenamin until the urine contains no pus and no. bacteria. Even after that point, observations on the condition of the urine should be made for several months, in order to be sure that the infection has died out.

Sequelæ of Pyelitis.-In the course of a few weeks the infection either clears up, becomes chronic, or destroys the kid- 
ney. The continuation or progression of the infection is manifested by fever; dry, red tongue; septic appearance; high white count and tenderness and enlargement of the kidney. The organ may be difficult to feel because of muscle spasm.

Under these circumstances operative interference is demanded. The question whether simple decapsulation, nephrotomy, or nephrectomy is needed must be answered at the time of operation.

If the infection apparently clears up, the physician, before discharging the patient, should obtain negative cultures from the urine. These should be obtained a week or two after the patient has ceased taking hexamethylenamin. If this is not done, a condition of chronic bacilluria may continue and menace the future health of the patient.

If bacilluria continues, whether or not accompanied by pus, the cause of the infection should be investigated and, if possible, cleared up. As a rule, the continuation of renal infection indicates some underlying abnormality of the kidney, such as the presence of stone or deficient drainage of the renal pelvis due to kink of the ureter. There is some evidence to show that movable kidneys are unusually susceptible to chronic infection, though whether this is due to obstruction to the outflow of urine or to congestion of the kidney is not known.

Investigation of such cases means $x$-ray to rule out stone, and cystoscopic study of the renal pelvis. Is it dilated? Is there obstruction? Is the kidney one of those severely in- 
fected, partially destroyed organs from which recovery cannot be expected? These questions can only be answered by careful study with cystoscope, renal function tests, and perhaps pyelography.

The treatment depends upon the cause. A thorough trial of hexamethylenamin must be made. In the case of infected movable kidneys associated with ptosis excellent results have been obtained in occasional instances by the use of proper ptosis corsets (Cabot and Brown).

A persistent pyelitis in which no other definite lesion of the kidney is found may be cured by pelvic lavage. Simons has collected from the literature reports of 66 cases so treated, which were sufficiently studied to be of value. Of the 66, 12 were cured by one lavage, 13 by two, 34 were cured only after three or more; 7 were not cured by lavage. Although the drug used is apparently unimportant, most urologists now employ silver nitrate in solutions varying from 1 to 5 per cent.

The use of vaccines in the treatment of renal infections has not given very striking results. Hugh Cabot, in a series of 30 cases of infection of the urinary tract, found that the use of vaccines was followed by improvement of the symptoms in more than half the cases, but had little effect on the bacteriuria.

If a definite abnormality, such as obstructed pelvic outlet, exists, it must be relieved by operation. If the kidney is of the sclerotic type, the parenchyma largely replaced by scar tissue, this fact will be indicated by a persistently low function. Such a kidney should be removed, provided the other 
kidney is healthy. It not infrequently happens that the process is bilateral; in that case removal of either kidney, provided it has any function whatever, is not proper unless nephrectomy is made necessary by the trouble caused by that kidney.

Bilateral Chronic Pyelitis.-M. A. L., masseuse, aged forty-five, was sent to me in March, 1913, complaining only of foul urine. 'This condition she thinks has always been present since she was fourteen years of age, at which time she had "summer complaint." When eighteen years old she had a suprapubic cystotomy for stone, but none was found. She has never had pain in the bladder or in the kidney region. She has never passed blood, and has no frequency or burning on urination. She gets tired very easily, but otherwise is in fair health.

Physical examination: A tall, thin woman. Heart and lungs normal. Abdominal palpation shows that on deep inspiration the entire right $k$ idney and the lower half of the left kidney are palpable. $x$-Ray shows no evidence of stone. Both kidncys are distinctly outlined. The urine is cloudy and ammoniacal, with pus, and culture shows the colon bacillus. No resilual urine. (ystoscopy shows a patchy cystitis. On the right side of lower lip of the internal meatus is a shallow diverticulum. Both ureters appear normal. The specimen from the right kidney shows epithelial cells and a negative culture; that from the left shows a few round and caudate cells and colon bacilli.

On June 6 and 27, 1913, the pelvis of the left kidney was washed with $1: 1000$ silver nitrate solution. ()n July $3 \mathrm{~d}$ the right kidney became tender, although the patient was not sick. July 11 th both ureters were catheterized. From the right much epithelium, no pus, colon bacilli. Urine from the left was hazy with bacteria. Phthalcin test: The injection was partly but not entirely intravenous. Color appeared on both sides in five minutes. The secretion from the right kidney was much better than that from the left. July 17 th the right kidney pelvis was washed with silver nitrate $(1: 8000)$; the urine from that side being perfectly clear.

The patient was not seen again until January, 1915. She had gained 5 pounds in weight. The urine was slightly hazy, but not foul or ammoniacal. Cystoscopy: Bladder clean. Urine from right, no albumin, pus, or bacteria; left hazy, no pus, many colon bacilli. Phthalein 1 c.c. intravenously. Color appeared on right in three minutes, on left in 


\section{AN OUTLINE OF GENITO-URINARY SURGERY}

seven minutes. There was much stronger color on the right, but so much leakage into bladder that estimation was not done. Both pelves washed with silver nitrate $(1: 5000)$. Capacity of left renal peivis 42 c.c.

In May, 1915, patient weighed 127 pounds-a gain of 7 pounds. Urine was still hazy, albumin slightest possible trace. She complained of feeling tired all the time, and had some vague pains in the joints. July 2, 1915, catheteriźation of ureters showed no pus from right, slight

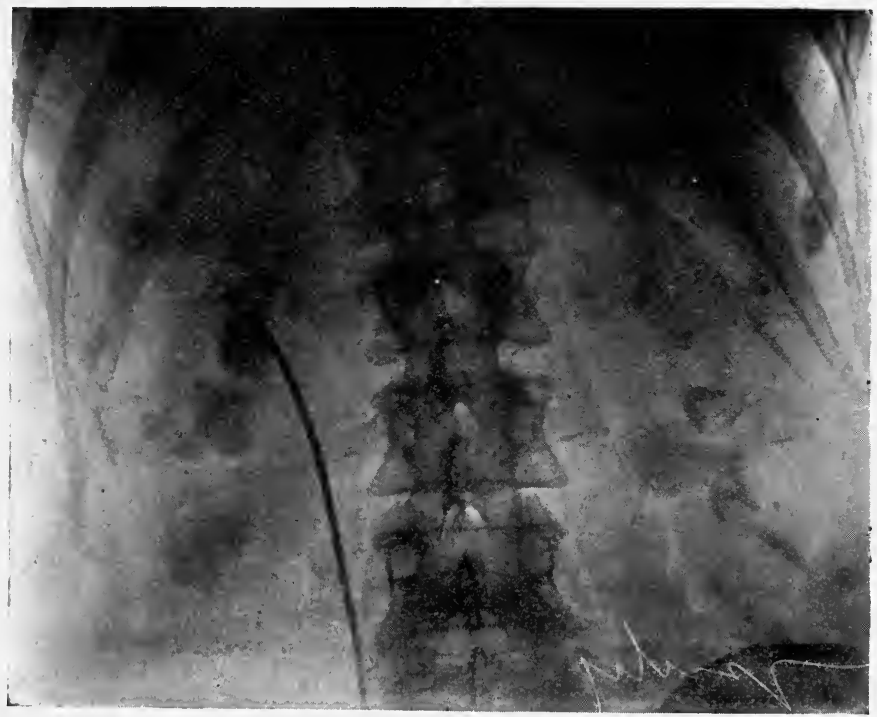

Fig. 63.-Chronic pyelitis with dilatation of both renal pelves. The radiographic catheter is in the left ureter (Mass. General Hospital). (See Case M. A. L.)

growth of colon bacillus; from left, no pus, profuse growth of colon bacillus. Phthalein: Appearance time, right three minutes, left four minutes. In fifteen minutes 9 per cent. excreted from right, 4 per cent. from left. None in bladder. Urea: Right, 2.4 per cent.; left, 1.9 per cent. A brief course of autogenous colon vaccine was given, with definite relief of the pains in the joints. On February 8, 1916, pyelograms of both kidneys were made (Fig. 63). Both showed dilated pelves. December 8, 1916: Has had no treatment for past year. Weight 125 pounds. Complains of 
feeling tired, but is able to work. The urine is almost clear and the kidneys seem distinctly less movable.

This case is interesting from several points of view. It illustrates the gradual attenuation through which a colon bacillus infection will pass. At first the bladder reacted to the infection; later it recovered. The kidneys, with pelvic dilatation due probably to their mobility, could not throw off the infection. The right kidney suffered little, the left showed marked destruction of tissue, as evidenced by the furctional test and considerable dilatation of the pelvis.

Renal Retention.-Improper drainage of the renal pelvis may be due to the kinking of the ureter by adhesions between it and the lower pole of the kidney or by aberrant vessels which draw it into an acute angle. Mobility of the kidney alone is not enough to cause obstruction to the urinary outflow; the ureter must be fixed at some point in its upper part in order to become obstructed. The chief symptom of obstruction of this type is intermittent pain, very severe, perhaps accompanied by vomiting, and at times relieved by posture or by pressing the kidney upward with the hand. If the obstruction is severe enough and frequent enough to cause hydronephrosis, the later phase is generally painless.

Hydronephrosis Due to Aberrant Vessels.-Miss E. F., age twentythree, was sent to me November 9, 1917. She had always been well until last winter. In February she had had tonsillitis, the following June had attacks of backache and fainting spells. Her doctor told her she had pyelitis. She lost 16 pounds in two weeks. She has had constant backache since then, and has felt poorly whenever she attempts to go back to her work as clerk. Frequency not abnormal. $x$-Ray negative for stone.

November $23 \mathrm{~d}$ she entered the Deaconess Hospital. Her evening 


\section{AN OUTLINE OF GENITO-URINARY SURGERY}

temperature was $102.4^{\circ} \mathrm{F}$. The urine was cloudy, containing much pus and colon bacilli. Cystoscopy showed a clean bladder and normal appearing ureters. Both were catheterized, and from the right a dilute, hazy urine containing pus-cells, bacilli and cocci, was obtained; from the left a concentrated, hazy urine, containing blood and epithelial cells; no

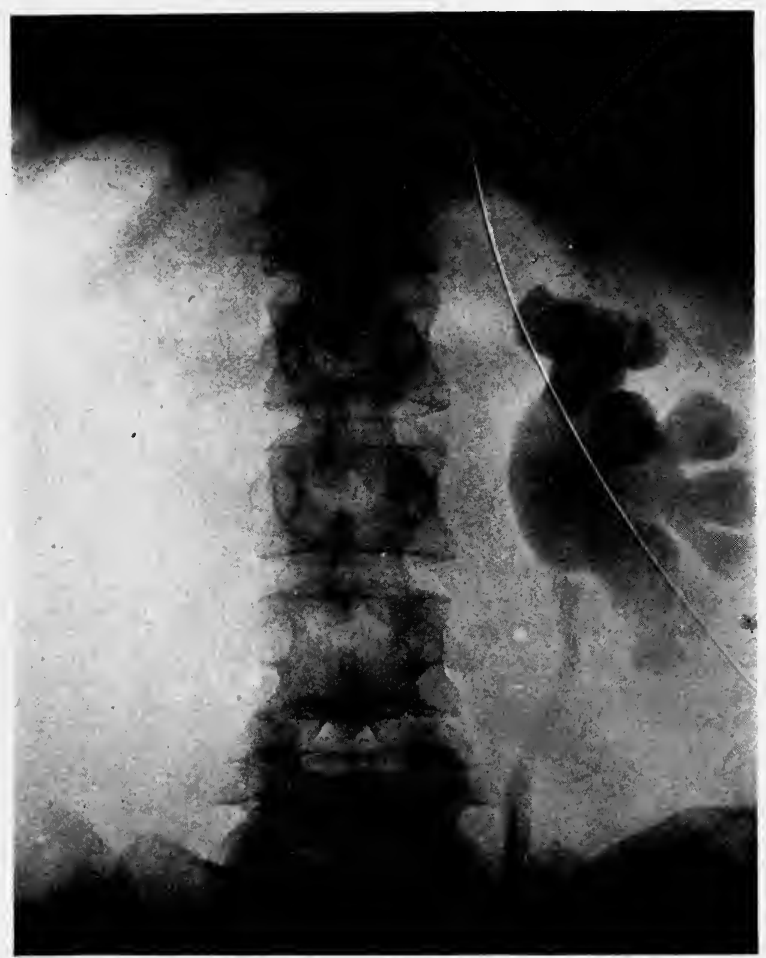

Fig. 64.-Renal retention, infected, due to kinking of ureter at pelvic outlet by aberrant vessels. ( $x$-Ray by Dr. L. B. Morrison.)

bacteria or pus. Divided function, phthalein intravenously: Appearance time on right three minutes, left, five minutes; 8 per cent. on right, 15 per cent. on left was excreted in fifteen minutes: no leakage into blad-. der. The pelvis of the right kidney held 25 c.c. of thorium. The pyelogram showed an abrupt transition between a dilated pelvis and a narrow 
ureter (Fig. 64). The diagnosis of hydronephrosis with ureter obstructed by aberrant vessels was made and the patient was operated upon.

At operation a fairly sound kidney, with much extrarenal dilatation of pelvis, was delivered. An aberrant artery and vein were plainly visible extending from lower pole of kidney behind ureter toward middle line of body. These were tied and cut, and adhesions about the lower pole of the kidney were freed. The pelvis was opened, and a bougie passed down ureter, showing no further obstruction. I rubber tube was passed through cortex of kidney into the pelvis. Edges of incision into pelvis were loosely approximated and kidney replaced. Convalescence uneventful. Two weeks after operation the tube was removed. A leakage through the sinus stopped almost immediately. Temperature remained normal and the patient had no further pain. She gained rapidly in weight, and when last seen on January 15, 1918, the urine was clear and free from albumin.

For the cure of renal retention operation is required, and may consist of freeing the ureter, of a plastic between pelvis and ureter, or if the kidney is largely destroyed, of nephrectomy (Fig. 65).

Renal and Ureteral Stone.-Calculi in the upper urinary tract are formed by the deposition of crystals about a nucleus. This nucleus may consist of bacteria or other organic matter, but often stones form in otherwise normal kidneys, and it is believed that they form upon nuclei composed of uric acid crystals cemented together by the colloid matters, such as pigment, which occur normally in urine. The commonest stones are those composed chiefly of oxalates; the next in frequency are the phosphatic stones. Calculi of uric acid or calcium urate are much less frequent. Rare stones are found composed of cystine or xanthin. Oxalate stones form rather slowly, as far as we can judge from the rarity of their recurrence. Phosphatic stones, on the other hand, may' 
re-form within a year or two after removal, and in such cases are a serious menace to life. For, although renal stone may

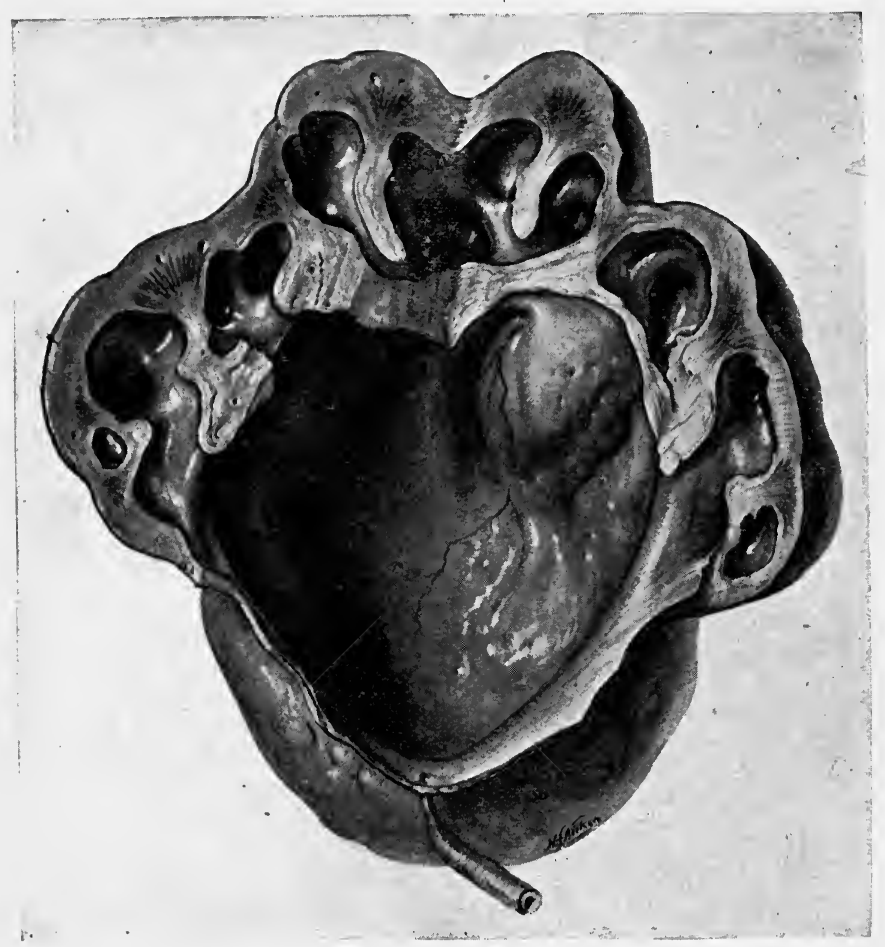

Fig. 65.-Hydronephrotic kidney, split longitudinally. From a woman aged thirty who for two years had had severe attacks of pain in the region of this kidney. She was sent into the hospital with the diagnosis of gall-stones. Radiograph showed an enlarged kidney containing two small stones. Ureter catheter drew off 40 c.c. of brownish fluid. Phthalein output was negligible. (Cambridge Hospital.)

exist in a kidney for years witbout inflicting serious damage, it is sure to increase in size, and sooner or later will injure the kidney in which it is harbored. Stones damage a kidney in 
two ways: (1) By obstructing the outlet; (2) by causing lesions in which infection gets a foothold. These two factors, by working together, produce the typical pyonephrotic stone kidney - a mere shell divided into five or six compartments, each of which represents a calyx dilated to such an extent that the parenchyma has entirely disappeared.

There are no typical symptoms of stone. Renal colic, which means the blocking of pelvic outlet or ureter, is always strongly suggestive, and if accompanied by blood in the urine it makes the diagnosis of stone very probable. It is not infrequent for renal stone to be absolutely painless.

Renal Stone-Hematuria the Only Symptom.-M. M. IV. Massachusetts General Hospital, No. 210,430. Age thirty-two, female, married; came to the Consultation Clinic with a history of having had blood in the urine for the past two months. Her past history was negative, and except for a dull ache in the region of the right kidney, which she felt after standing a long time, she had had absolutely no other symptoms. $x$-Ray showed a large stone in the right kidney (Fig. 66). Its outline closely followed that of a renal pelvis. Urine from right kidney was bloody, that from left clear. Both showed a few colon bacilli. Divided function after intravenous phthalein showed on right, five minutes appearance time, 1.25 per cent. in fifteen minutes; left, three minutes appearance time, 15 per cent. in fifteen minutes. Pyelogram on right suggested destruction of the calices. Nephrectomy was done. The pathologist reported the calices considerably dilated, the pelvis pale, with small areas of hemorrhage. It was filled with a branched calculus weighing $3.1 \mathrm{grams}$, and four small calculi weighing 0.8 gram. All were of calcium oxalate. Microscopic examination showed essentially normal kidney tissue, with areas of round-cell infiltration and fibrous thickening underlying the pelvic mucosa.

The position and size of the stone must be demonstrated by $x$-ray before operation (Fig. 67). It should be borne in mind that about 10 per cent. of all stones are undetected by 
$x$-ray examination; the diagnosis must then be made by the cystoscopist with wax-tipped catheter and pyelogram.

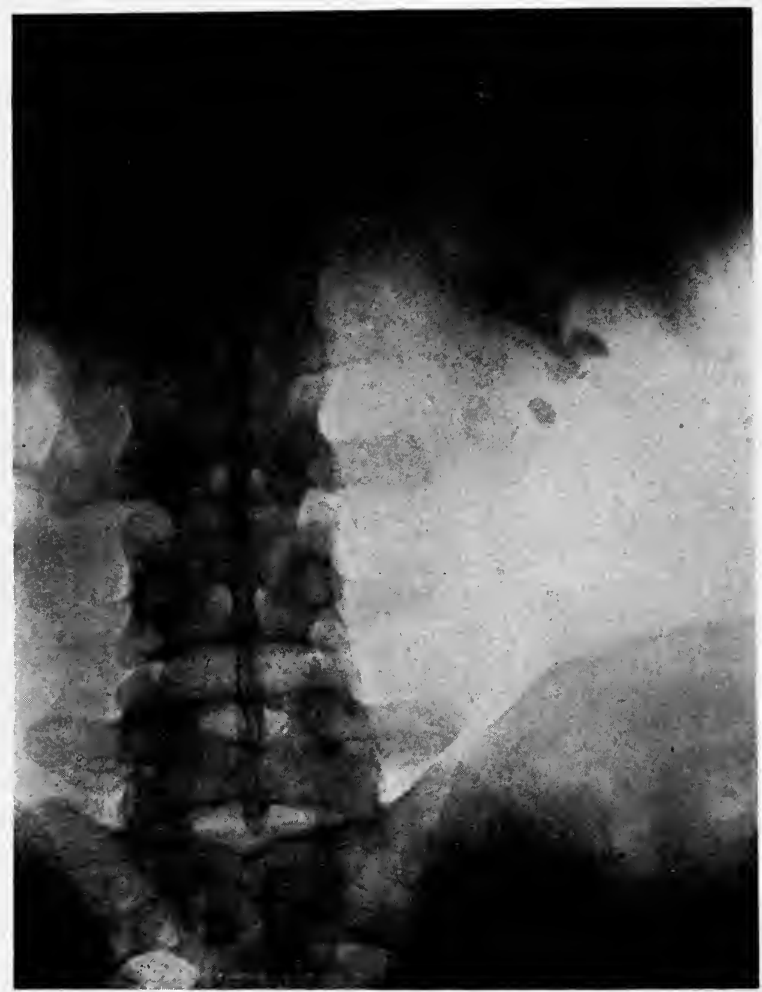

Fig. 66. - $x$-Ray showing renal stone. Only symptom was hematuria of two months' duration (Mass. General Hospital).

In the great majority of cases of renal stone operative removal is indicated. There are certain cases of bilateral stone, however, in which the damage caused by removal would be greater than that caused by the stones themselves. Such 
cases call for a thorough knowledge of the patient and of the renal data, and for the exercise of much surgical judgment.

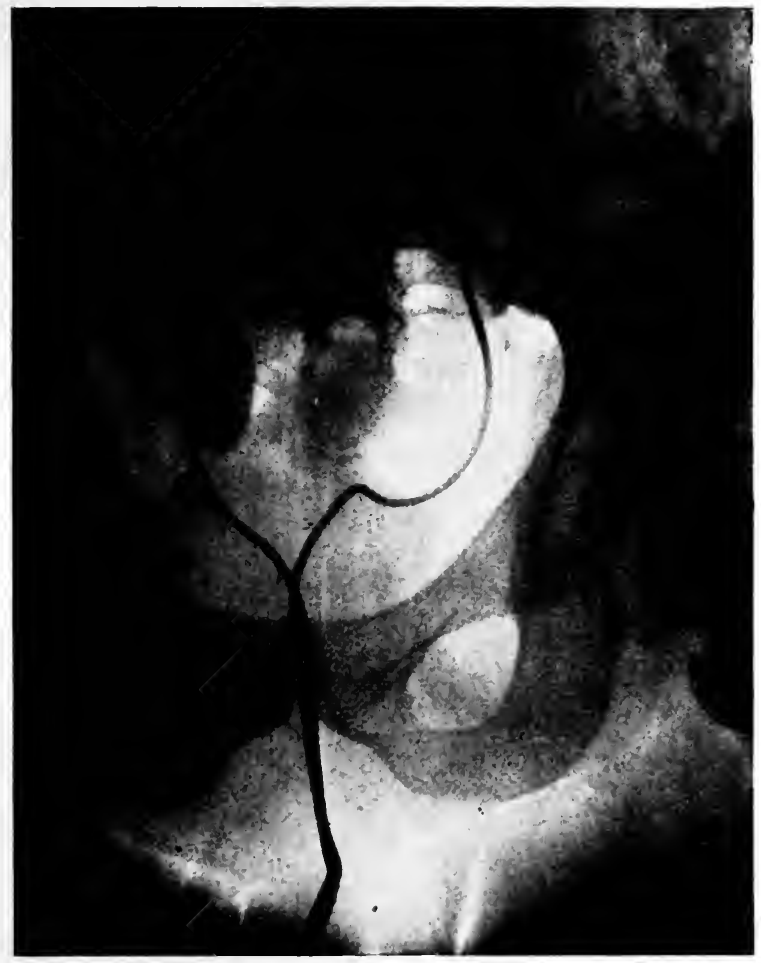

Fig. 67.-Ureteral calculus, later removed through an extraperitoneal Gibson incision (Mass. General Hospital).

Stones once formed, of course, cannot be dissolved, but if small, they may be passed. Ureteral stones as large as datepits occasionally come through, whereas, in other instances, the tiniest stones may lodge in the ureter indefinitely. 


\section{0 \\ AN OUTLINE OF GENITO-URINARY SURGERY}

The passage of ureteral stones can often be aided by the introduction of a ureteral catheter and the injection into the ureter of oil; sometimes it is necessary to slit the ureteral orifice through the operative cystoscope. A small ureteral stone, if not causing obstruction of the ureter, may be left

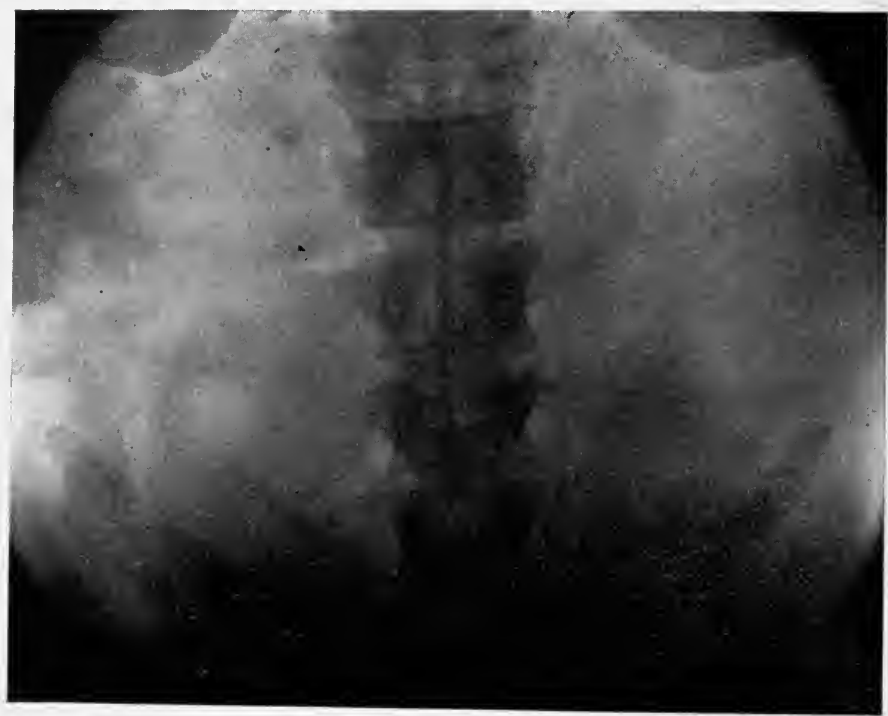

Fig. 68.-Shadow apparently in course of ureter (Mass. Genera] Hospital).

for months without injury to the kidney, in the expectation that it may be passed. If a second $x$-ray taken after several months shows that the stone has not moved, and cystoscopic measures have failed, it will be found that the stone is of the spiculated type, tightly grasped by the spasm of an irritated ureter. Operative removal is then the only way out. 
Ureteral Stone-Ureterolithotomy.-M. I. F., a woman of thirty-two, was sent to me on May 29,1916, complaining of headache and of acute pain in the right back, radiating down the leg to the foot. In 1913 her appendix had been removed, following an attack of pain similar to the present. During the past year she had had eight attacks of pain begin-

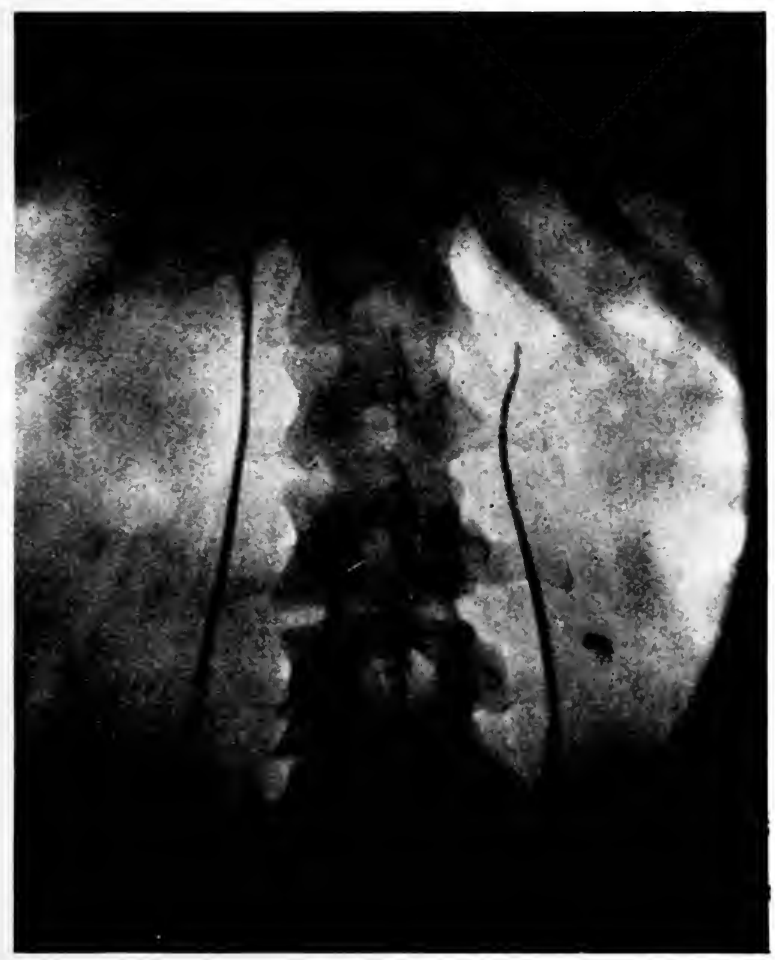

liig. 69.- Same shadow with radiographic catheter in place. Shadow is seen to be $\frac{1}{2}$ inch away from ureter (Mass. General Hospital).

ning in the right flank and radiating to pubes and to leg. Two attacks were accompanied by some hematuria.

General physical examination negative, except that the right kidney was easily palpable on deep inspiration; not tender or enlarged. Urine was clear, with the slightest possible trace of albumin. Sediment showed 


\section{AN OUTLINE OF GENITO-URINARY SURGERY}

epithelial cells; no pus or bacteria; quite a number of red blood-cells. $x$-Ray showed a shadow low in the right ureter (Fig. 70). June 6th, cystoscopy: A catheter was held up at about the situation of the shadow. Ureteral meatus was cut through the operative cystoscope; a No. 5 catheter was passed to the kidney and 3 c.c. of gomenol oil was injected above the stone. June 8th she had a bad attack of renal colic. June 19 th the ureteral orifice was stretched through the operative cystoscope,

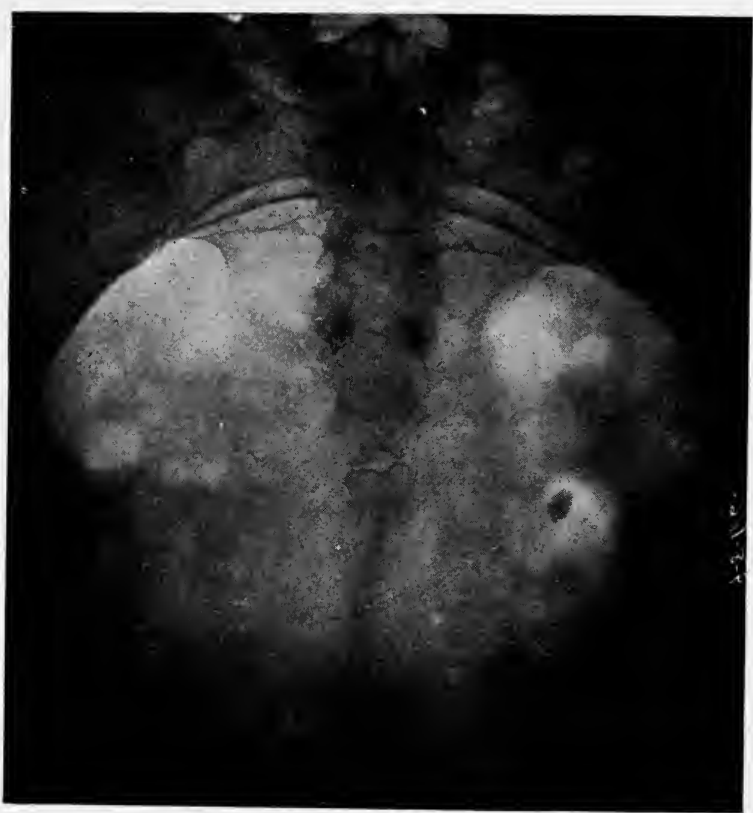

Fig. 70.-Stone low in right ureter. Removed by ureterolithotomy, as it was firmly impacted. ( $x$-Ray by Dr. W. J. Dodd.)

and an attempt made to seize the stone with nippers, but without success.

September 12, 1916, as the patient had had several attacks of pain, and was nearly always conscious of discomfort, operative removal of the stone was decided upon. Through a right-sided Gibson incision, extraperitoneal, the ureter was found moderately thickened and dilated. An oval, black stone was pressed up the ureter and removed through a small 
incision at the pelvic brim. One rubber tissue drain. The wound healed by first intention without urinary leakage.

May 3, 1917: The patient has been South all winter and now feels very well She still has occasional pain in the right kidney, and the bladder urine shows the slightest possible trace of albumin; a number of pus-cells, and some colon bacilli; no blood or casts. Patient was put on 15 grains of hexamethylenamin three times a day. June 11th the urine showed no albumin and only a rare pus-cell. July oth, cystoscopy: No. 6 catheter passed easily to the right kidney. Urine showed a few red blood-cells and epithelial cells; no pus at all. Pelvis washed with 1 per cent. silver nitrate. Since then the patient has had no symptoms referable to the kidney.

During the renal or ureteral colic of stone the patient will get considerable relief from the application of heat, but severe attacks require a hypodermic injection of morphin. Atropin is supposed to be valuable through the relaxation of smooth muscle which it causes. There is experimental evidence to show that papaverin or the almost forgotten Sahli's mixture are more effective than morphin in relieving the ureteral spasm.

The prevention of the recurrence of stone after operation or after spontaneous passage consists in keeping the urine very dilute and in eating food which will decrease the excretion of the salt which was most concerned in the formation of the stone.

In the case of oxalate calculi, which form slowly and which do not tend to recur, the urine should be watched for the occurrence of crystals of calcium oxalate. If the content of oxalates is found to be high, the patient should avoid foods which contain much oxalic acid, such as cabbage, spinach, aspáragus, apples, and grapes. If this does not produce the 
oxaluria, other dietetic measures should be instituted. The physiologic chemistry of oxalic acid has not yet been thoroughly determined, but there is evidence to show that gastric fermentation may increase the output of oxalic acid by the kidneys.

Phosphatic calculi are particularly prone to recur. In those cases in which they re-form within a year or two after removal, the disease may well be called "malignant."

Recurrent Renal and Ureteral Stone.-G. S. A., an Albanian, twentythree years of age, was admitted to the Massachusetts General Hospital May 23, 1914, because of attacks of right-sided pain. $x$-Ray had showed a small shadow in region of right ureterovesical junction, and cystoscopy had confirmed this finding. By combined intra- and extravesical routes I removed a small stone from the lower end of the right ureter. Except for a peri-urethral abscess convalescence was uneventful.

After leaving the hospital he continued to have attacks of right-sided pain. Investigation showed that the lower end of the right ureter was strictured so tightly that not only did a filiform fail to enter, but indigocarmin was not excreted from that side. December 19, 1914, I resected his right ureter 1 inch above its entrance into the bladder and reimplanted it. Convalescence was again uneventful.

In September, 1916, he was seized with severe pain in the left side and ran a temperature of $102^{\circ}$ to $103^{\circ} \mathrm{F}$. $x$-Rays showed three small shadows low in left ureter, one larger shadow high in the left ureter, and one in the left pelvis. The left kidney was large and tender, and he was manifestly very sick. I cut down upon his left kidney, which was large and congested, and removed a small stone from the pelvis and a larger one from the upper ureter. The kidney was drained by a tube passed into the pelvis through the cortex. Good recovery, with prompt relief of pain and fever. Two weeks later I removed the three stones from the lower left ureter through a Gibson incision.

Following this he was very well for about a year. He followed carefully the diet outlined on page 255 . In spite of that, an $x$-ray taken in the fall of 1917 showed a small shadow in the left kidney region. In July, 1918, after a careful metabo'ic study. which showed that the phosphatic content of his urine could be greatly reduced by diet, I removed this stone also. Good convalescence. 
The stones in such cases are frequently bilateral, and unless their formation can be stopped, surgery in itself cannot prevent the destruction of the kidneys. It has been shown that in such a case the phosphatic content of the urine may be three or four times the normal, and that this excess may be substantially reduced by proper diet.

The foods to be avoided are eggs, milk, and fish. It has been shown that the ingestion of calcium carbonate precipitates the phosphates in the food as insoluble salts and thereby prevents their absorption. In addition to avoiding the above-mentioned foods, therefore, one should take a dram of calcium carbonate after each meal; this has a constipating effect, but may be kept up for years without damage to the organism.

Renal Tumor.-Pathologic enlargement of the kidney may. be due to hydronephrosis, single cysts, cystic degeneration (so-called polycystic kidney), echinococcus cyst, or to the presence of a solid tumor. The great majority of solid tumors of the kidney are of two types: (1) the embryonic tumor, which is discovered within the first three years of life, and (2) the hypernephroma of middle life, which appears generally between the ages of forty and sixty. Rarer tumors are papillomata of the renal pelvis, papillo-adenocarcinomata, flat-celled carcinomata, and sarcomata.

Tumors of the embryonic type usually attract attention through the noticeable increase in size of the child's abdomen. Blood in the urine clinches the diagnosis. The tumor is smooth, only slightly movable, and of elastic consistency. 


\section{AN OUTLINE OF GENITO-URINARY SURGERY}

Pathologically, it consists of tissues derived from connective tissue and from epithelium; the former give rise to areas which resemble sarcoma; the epithelial tissue may present abortive attempts at the formation of tubules. What is left of the kidney is compressed into a crescent of renal tissue, borne upon one aspect of the growth. As soon as the tumor is discovered it should be removed. The prognosis is poor.

Hypernephroma, the common renal tumor of adult years (80 per cent. of all renal tumors according to Wilson), was believed by Grawitz to develop from rests of adrenal tissue which were included by the kidney during its development. Stoerck attacked this theory and suggested that the neoplasm arose from regenerating convoluted tubules in the atrophic kidney. Wilson proposed the hypothesis that hypernephromata are derived from islands of nephrogenic tissue which have failed to become connected with the renal pelvis through the collecting tubules of the developing kidney. The commonest symptom, occurring in 28 cases out of 32 (Wilson), is hematuria, which may appear before the renal enlargement is perceptible to palpation. This is the time for operation, so that the tumor may be removed, if possible, before metastases have taken place. The diagnosis is made by cystoscopy, aided by separate renal function and pyelography. The encroachment upon the renal pelvis by the tumor causes a deformity of outline, sometimes characterized by the "spiderweb" appearance, sometimes by an indentation in the pelvic outline (see Fig. 59). Once the diagnosis of hypernephroma is made, nephrectomy is indicated unless there is evidence of 
metastasis in lung or long bone, or the patient is cachectic and clearly overwhelmed by the malignancy of the growth.

Polycystic kidney may give a characteristic knobby sensation on palpation, and, as the process is bilateral, the total renal function is generally low. There are signs of chronic nephritis. Echinococcus cysts in the kidney give only the signs of tumor until they rupture. After that, daughter cysts appear in the urine. Pyelography shows that the tumor is renal. The blood should contain a definitely ir creased number of eosinophils and should give a positive complement-fixation test with echinococcus antigen. Nephrectomy is indicated.

Rupture of the kidney is generally caused by a fall in which the patient strikes his flank upon a sharp edge, or by a blow over the kidney. I have seen a kidney ruptured by a shrapnel ball. The projectile had torn the pelvis away from the kidney and had wrapped the pelvis about itself. The prominent symptom of rupture of the kidney is hematuria. In some cases the extravasation of blood about the kidney fills the fatty capsule with clot and forms an easily palpable mass.

If the bleeding is not severe the patient should be put to bed and kept absolutely quiet for from one to two weeks. A certain number of cases will get well with this treatment alone. Some will continue to bleed, will run a temperature, and will clearly demonstrate the necessity of operative interference. Others, when seen at the very start, will show the signs of rupture impossible to heal. There may be great 
shock or the signs of extensive hemorrhage. If the former, the patient's condition must be improved before operation is done.

Operation usually consists of nephrectomy. Once in a while a kidney is found which may be sutured with some hope of getting a useful organ. The interposition of strips of muscular tissue between the torn surfaces acts as a hemostatic (Risley).

Rupture of the Kidney-Nephrectomy.-A. L., a brakeman, twentyfour years of age, was caught between a freight car and a projecting roof. He was taken to the hospital at once. The first urine voided was clear, the second specimen was bloody, and the urine remained bloody for two weeks. The bleeding then apparently stopped. A few days later, after reaching for a glass of water, he had a very severe hemorrhage, filling the bladder with clot. I saw him two days later. His pulse was 105 to 110 , his temperature $100^{\circ}$ to $101^{\circ} \mathrm{F}$. His bladder seemed moderately distended (clots) and he had a large mass in the right flank. Immediate operation was done. The fatty capsule was distended with blood-clot. The kidney was found to be torn almost in half and the pelvis was split wide open. The peritoneum was opened and the other kidney palpated, after which the damaged kidney was removed. Convalescence was uneventful.

BraAsch, W. F.: Clinical Data of Multiple Cystic Kidney, Surg., Gynec., and Obst., 1916, xxiii, 697.

САвот, H.: Diagnosis and Indications for Operation in Early Hydronephrosis, Jour. Amer. Med. Assoc., 1913, lx, 16-20. Treatment of Movable Kidney With or Without Infection by Posture, Boston Med. and Surg. Jour., 1914, clxxi, 369-373. Stone in the Kidney and Ureter. A Critical Review of 157 Cases, Jour. Amer. Med. Assoc., 1915, lxv, 1233.

Caвot, H., and Crabtree, E. G.: Classification and Treatment of Kidney Infections, Boston Med. and Surg. Jour., 1916, clxxiv, 780. Etiology and Pathology of Non-tuberculous Infections of the Kidney, Surg., Gynec., and Obst., 1916, xxiii, 495.

Cunningham, J. H.: Acute Unilateral Hematogenous Infections of the Kidney, Trans. Amer. Assoc. Genito-urin. Surg., 1912, vii, 145-169. 
EIsexdratu and Kalin: Rôle of Lymphatics in Ascending Renal Infection, Jour. Amer. Med. Assoc., 1916, cxvi, 561.

Geragity, J. T.: The Treatment of Chronic Pyelitis, Jour. Amer. Med. Assoc., 1914, Ixiii, 2211-2214.

Keyes, L. L., JR.: Mechanics of Renal Infection, Lancet-Clinic, 1916, $\mathrm{cxv}, 121$.

Morse, J. L.: Edebohl's Operation in Nephritis in Children, Jour. Amer. Med. Assoc., 1917, lxix, 525-530.

Schmid, L. E.: Nephrectomy During Pregnancy, Trans. Imer. Assoc. Genito-urin. Surg., 1915, x, 109-119. Echinococcus of the Killney Trans. Amer. Assoc. Genito-urin. Surg., 1915, x, 129-143.

Smitı, (i. (i.: Renal Stone, Boston Med. and Surg. Jour., 1917, clxxvi, $524-529$.

Smitn, R. Ml: Recent Contributions to the Study of Pyelitis in Infancy, Amer. Jour. I)is. Children, 1913, $1,273-278$.

Sequer, J. B.: Renal Lithiasis, Imer. Jour. Surg., .Ipril, 1913. Renal Pain: Diagnostic and Clinical Significance, Trans. Amer. Assoc. Genito-urin. Surg., 1915, x, 265-282.

Wilson, L. B.: The Embryogenetic Relationship of Tumors of the Kidney, Suprarenal, and 'Testicle, Inn. Surg., April, 1913. Iypernephromata, Old Dominion Jour. Med. and Surg., 1910, x, No. 4. 


\section{CHAPTER XIV}

\section{GENITO-URINARY TUBERCULOSIS}

THE tubercle bacillus strikes the male genito-urinary tract chiefly at two points-the kidney and the epididymis. In the female renal tuberculosis appears as in the male; genital tuberculosis is less frequent than in the male, and has less to do with the urinary organs.

Strictly speaking, tuberculosis of the genito-urinary tract is never primary, but depends upon the existence of a focus elsewhere in the body. This focus may be a single tuberculous gland, or may consist of extensive lesions of bone or lung. The route by which the kidney becomes infected has been in much dispute. Lawrason Brown has shown that the kidney can filter out tubercle bacilli presumably from the blood without damage to itself. That it sometimes fails in this, and suffers from the lodgment of bacilli in its own tubules, is one explanation of the method of infection.

The lymphatic route is considered by some urologists to be the more probable. Brongersma showed that particles of dust could pass from peribronchial lymph-rodes against the stream to the juxta aortic nodes, and believed that either in this way, or from infected mesenteric nodes, the tubercle bacilli reached the kidney. In defence of his theory he presented a number of cases in which the pulmonary and renal involvement were upon the same side. 
Kidney and Bladder.-Perhaps according to the route by which the bacteria reach the kidney, two types of lesions appear. One is the pelvic type, in which the pelvic mucosa is much involved, and the apices of the pyramids ulcerated and nibbled off by the bacteria. The other is the cortical type, in which the pelvis is little involved, at least at first, and in which abscesses develop in the parenchyma of upper or lower pole.

From these foci bacteria descend with the urine, and after the process has proceeded for some time, they succeed in securing a foothold in the bladder mucosa. Buerger has diagnosed early renal tuberculosis by finding the organism in a bit of edematous mucous membrane snipped from the bladder wall just below the ureteral orifice on the infected side. Only when the bladder becomes involved does the patient awaken to the fact that all is not well with him. Renal tuberculosis is nearly always painless. It is the bladder involvement which troubles the patient. Rarely the presence of considerable blood in the urine is the first symptom; as a rule, the history of a case of renal tuberculosis is one of slowly increasing bladder irritability.

If the process continues, the bladder becomes more completely involved, the wall thickens, scars distort the interior. The affected ureter becomes shorter and dilates; the lower end of the other ureter becomes thickened and indurated, and the infection spreads, presumably by the lymphatics of the ureter, into the second kidney.

No medicines avail. No case of renal tuberculosis has 
ever been proved to be cured except by nephrectomy, either at the hands of the surgeon or brought about by total destruction and gradual dissolution of the kidney.

Tuberculosis is never primary in the bladder. The diagnosis of uncomplicated renal tuberculosis is, therefore, within the reach of every practitioner. If there are no genital lesions, pus and tubercle bacilli in the urine must come from the kidney. As a general rule, the urine in renal tuberculosis does not contain other bacteria; if the stained sediment from a catheter specimen of urine shows pus and no bacteria, one should suspect tuberculosis, and have the urine inoculated in a guinea-pig or examined microscopically. This is done nowadays by many laboratories, boards of health, and medical schools, so that the physician without laboratory facilities need not lack the help which a test of this sort will give him.

The study of the renal condition must be done through cystoscopy. Often anesthesia will be required, and in this event spinal anesthesia is admirable. It is often impossible to tell, without cystoscopy, which side is affected. The healthy kidney hypertrophies; pain may be caused by the stretching of its capsule, and the enlargement may be perceptible on palpation. Surgeons misled by the pain and enlargement have been known to remove the hypertrophied normal kidney, leaving a kidney normal even to intra-abdominal palpation, but absolutely destroyed by tuberculosis.

If one kidney alone proves to be infected, it should be removed. A careful study by Israel of patients nephrecto- 
mized for various causes showed that the prognosis was distinctly good; that one of the earliest cases was still alive fifteen years after the operation, and had borne several children. Of the cases nephrectomized for tuberculosis in the series collected by Cabot and Crabtree, over 50 per cent. were permanently improved.

The operation should be followed for months or even years by careful hygiene and injections of tuberculin. Our experience at the Massachusetts General Hospital has demonstrated the value of these measures. About 25 per cent. of all cases develop sinuses in the nephrectomy wound, and the healing of these is greatly hastened by proper after-treatment, i. e., hygiene, direct sunlight, and tuberculin. Nearly all of these cases have infected bladders; when the main focus of infection, which is the kidney, has been removed, the bladder ulcerations get a chance to heal.

The process of getting well, so far as the bladder is concerned, is divided into two parts: First of all, the ulcerations must heal. This process is aided by the administration of sandalwood oil, 10 minims in a capsule, one to be taken during each meal; hexamethylenamin has no curative value in tuberculosis and serves simply to irritate the hypersensitive bladder. Healing is further aided by the instillation into the bladder of some therapeutic agent. In our experience the best results have been secured by the use of gomenol oil. One ounce is injected into the empty bladder once or, better, twice a week. Good results have also been obtained by the instillation of increasingly strong solutions of carbolic acid- 
1 ounce of $\frac{1}{2}$ per cent. solution is used at first, and is injected twice a week. If no reaction is evident, a 1 per cent. carbolic acid solution should be used. The strength of the solution may be gradually increased to 5 per cent., but should always be kept so low that the reaction, which is manifested by slight hematuria, does not last for more than twenty-four hours.

After the ulcers are healed the bladder will frequently be found to be contracted by scar tissue. The gradual dilatation of the bladder forms the second stage in the treatment of tuberculous cystitis. About twice a week it should be filled with fluid until the patient complains of the distention. The capacity should increase $\frac{1}{2}$ ounce at a time. For distending the bladder a very weak solution of corrosive sublimate ( $1: 20,000$ to $1: 10,000$ ) may be employed. The mercuric chlorid influences favorably whatever remains of the tuberculous ulcerations.

The presence of other foci of tuberculosis need not necessarily contraindicate operation. Frequently other lesions improve after nephrectomy when the toxemia from the renal process is removed. The possibility of stirring up a latent pulmonary infection is best prevented by the use of gasoxygen as an anesthetic.

If the disease should prove to be bilateral when first seen by the cystoscopist, the prognosis is poor. The disease may have remissions, but will within a few years reduce the amount of kidney tissue to a point where it is insufficient to support life. One is surprised at the degree to which this 
process of kidney attrition may be carried. One-fourth of the normal amount of kidney tissue will support life very well, and if the process of destruction is slow, life may be maintained with considerably less tissue than that in commission.

The worst aspect of bilateral renal tuberculosis from the patient's point of view is the vesical condition. Frequency becomes so great that after a time incontinence results, the urine dribbling from the bladder, the capacity of which is only an ounce or two. When this stage is reached the patient suffers less pain, and is troubled mainly by the discomfort of a rubber urinal.

Operations for diverting the urinary stream from the bladder, such as ureterostomy, have been adrocated by Rovsing, but their value is questionable. Cystostomy gives relief from pain, but is a disagreeable feature in itself. The best of antituberculosis hygiene, the use of sandalwood oil, and instillation of gomenol oil are the chief measures to be employed in a case of bilateral renal tuberculosis.

Genital tuberculosis in the male is almost always primary in the epididymis. Koll and Cunningham have each reported 2 cases of primary prostatic tuberculosis, but the condition is rare. Tuberculous epididymitis begins insidiously; often it is noticed for the first time when a blow upon the testicle has attracted the patient's attention to that part.

Tuberculous Epididymitis Following an Injury.-H. S. N., teamster, age forty-one, married. Entered the hospital November 20, 1916. Past history unimportant, except for an attack of gonorrhea at the age of twenty, apparently of short duration. 
Two months ago, while getting out of a truck, he slipped and the side of the truck struck him in the groin. He had severe pain in the right testicle for half an hour, but no swelling at that time. Three or four weeks later, however, testicle began to swell. The swelling was accompanied by a drawing sensation, but by no real pain in testis. Micturition normal. Patient has no children; wife has had three miscarriages. Sex function normal.

Physical examination shows a healthy appearing man. Penis and left testis normal. Hydrocele on right. This was tapped and $1 \frac{1}{2}$ ounces of hazy fluid withdrawn. Upper pole of epididymis very much swollen, hard, and nodular. Suggestive of tuberculosis. Vas not enlarged. Prostate soft. Right vesicle apparently a little swollen and hard. Urine clear and clean; no albumin.

On November 27, 1916, an epididymovasectomy was done under local anesthesia. Epididymis found thickened at upper pole, the induration extending on to cord. Testis normal. Examination of cut sections of epididymis showed areas of normal tubules, some filled with brokendown cells and leukocytes. Adjacent areas showed necrosis, lymphoid infiltration, and some epithelial cells.

Patient made an excellent recovery and left hospital in six days. Examination, December 19, 1916, showed a very good result. Testis normal. No thickening in scrotum. Urine clear and clean; no albumin by heat,

In the preceding case acute tuberculous epididymitis apparently did follow injury. There is a possibility that the epididymis may be so prepared for the reception of wandering bacilli. Belfield has shown that the epididymis has an excretory function, a legacy from its origin in the wolffian body, and it has been generally believed that this is the reason why tuberculosis affects the epididymis and not the testicle. The testicle shows an amazing resistance to infection, even when the process in the epididymis is extensive enough to form sinuses.

In early tuberculous invasion of the epididymis we find a hard, smooth enlargement of one or both poles of the epididy- 
mis not vastly different from the enlargement which occurs in the subsiding stage of acute gonorrheal epididymitis.

The testis and epididymis are easily differentiated. The vas is thickened, especially at its lower end, and has a beaded feeling. Sometimes the outlines of the scrotal contents are obscured by the presence of fluid; this must be withdrawn before satisfactory palpation can be done. As a rule, the seminal vesicle upon the affected side is harder and thicker than its fellow.

If left alone, the process in the epididymis may form an abscess, which in time becomes a sinus; sometimes spontaneous healing takes place after the discharge of all the infected tissue through the sinus. This process occupies months or years; meanwhile the disease may extend to the other epididymis.

A tuberculous epididymis should be removed shortly after the diagnosis is made. The operation can be clone under local anesthesia; the epididymis is dissected off the testis and removed. The vas is freed from the cord, the lower end is grasped in a curved hemostat and carried as high as possible in the inguinal canal. The skin is incised over the point of the hemostat, the vas is drawn up, and as much as possible is removed through the small incision in the groin. The testis, cleaned of tuberculous adhesions and inflammatory masses, is replaced in the scrotum. Only very rarely does a testicle so treated require further operation.

The infection may extend from seminal vesicle into prostate and involve the mucous membrane overlying that gland. 


\section{AN OUTLINÉ OF GENITO-URINARY SURGERY}

This gives a picture similar to that of renal tuberculosis, namely, bladder irritability, ulceration of bladder neck, pyuria. To prove that the kidneys are not implicated is rather a difficult matter. The introduction of a cystoscope through a prostate so infected is not to be undertaken lightly. Under the circumstances normal kidney function is perhaps the best evidence obtainable that there is no serious renal involvement. The case should, after epididymectomy, be treated with sandalwood oil, tuberculin, and gomenol, and every few months the renal condition should be carefully checked up. The prognosis in tuberculous prostatitis is not bad; both epididymes may have to be removed, and the prostate gland may have to undergo destruction by the tubercle bacillus, but in time the infection should die out.

Radical removal of prostate and seminal vesicles has been practised, but seems rather an unsafe procedure. The opening up of tissues so rich in blood- and lymph-channels to the tuberculous pus appears to us rather risky. The chance of getting a tuberculous urinary fistula through the perineum is large. On the whole, conservative treatment of prostatic tuberculosis seems preferable in the large majority of cases.

Not infrequently one or the other kidney proves to be infected. The infection is usually established by the time the doctor sees the patient, so that it is difficult to be sure whether the renal or the epididymal infection was the first to appear. There is considerable evidence to show that infection of a seminal vesicle may ascend to the corresponding kidney. Whatever route the infection takes, the infected organ should 
be removed. Nephrectomy and bilateral epididymectomy, done simultaneously or a few months apart, have been quite constantly associated in our series of genito-urinary tuberculosis, and recovery from the infection has been the reward in a considerable number of such cases.

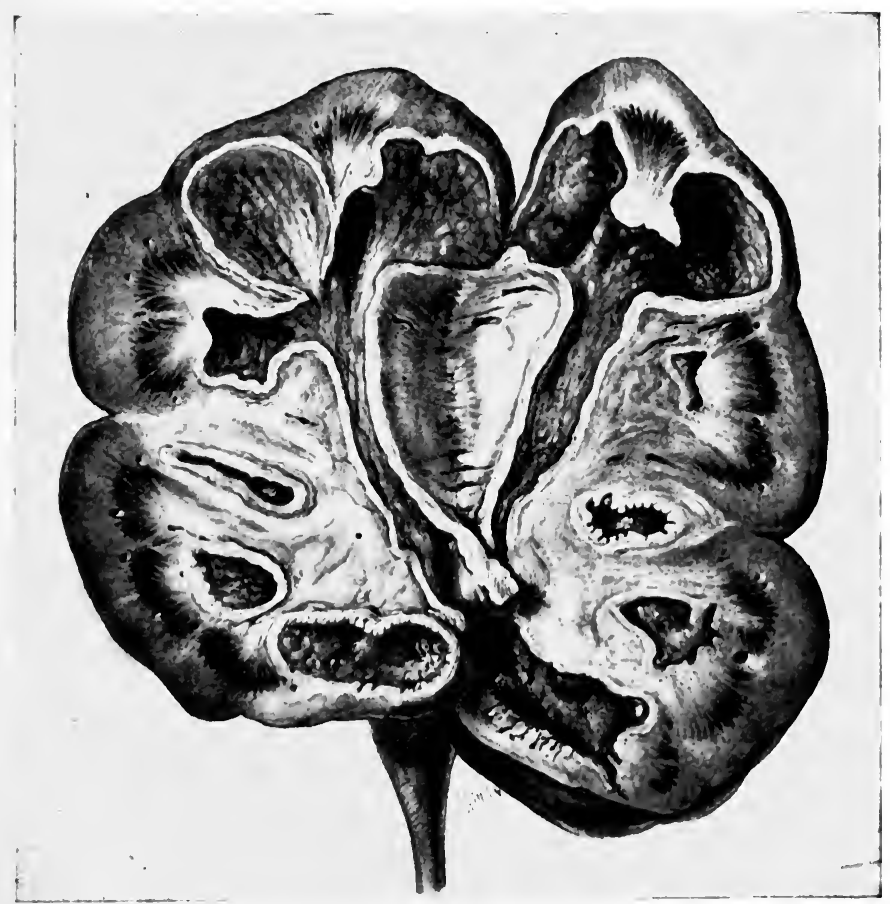

Fig. 71.-Tuberculous kidney removed at operation. History of pain in region of this kidney for four years. Bladder symptoms for past eighteen months. (See case report H. S.)

Genito-urinary Tuberculosis-Nephrectomy and Epididymectomy.H. S., a farmer, age thirty, single. Entered the hospital January 12, 1918. Family history and past history negative. About four years ago began 
to have attacks of severe pain in right flank, on one occasion requiring morphin. These attacks stopped for awhile; but came on again about three years ago. Then he began to be troubled with frequency. At times his urine contained blood. Lately he has had very little pain in right kidney, but bladder causes him a great deal of trouble. Frequency during the day every two hours or less; at night every fifteen minutes to one hour. Vibration causes pain in bladder. General health good. No particular loss of weight. Lost left testicle from unknown cause in early childhood.

Physical examination shows a well-developed and nourished man. Heart and lungs normal. Kidneys not palpable. Reflexes normal. Abdominal examination negative. Epididymis thick and hard, not very much so, but still definitely abnormal. Vas not enlarged. Urine cloudy; one large chunk of detritus or slough; albumin trace; sugar 0. Sediment (stained specimen), pus; a few bacilli and cocci. Cystoscopy: Bladder holds $2 \frac{1}{2}$ ounces. Typically tuberculous. Left ureter retracted, but orifice appears normal. Catheterized easily; clear urine obtained. Sediment shows rare blood-cell and epithelium. No pus. Phthalein: Appearance time four minutes, good output. Right ureter a gaping hole. Catheterized for $6 \mathrm{~cm}$. Backflow from bladder. Bladder specimen searched for tubercle bacilli. Some acid-fast organisms found.

January 17, 1918, right nephrectomy. Ureter found thickened. Kidney (after removal and splitting) shows typical cavities at upper and lower poles (Fig. 71). Right epididymectomy and vasectomy. Epididymis practically a cavity of thick pus.

Catheter left in bladder and bladder irrigated with 5 per cent. carbolic. Some breaking down of kidney wound superficially. The bladder was irrigated the third and fifth day after operation with carbolic ( 2 and 3 per cent.). Some improvement noted after catheter was removed.

To recapitulate: Genito-urinary tuberçulosis may be primary in kidney or epididymis. Not infrequently it appears more or less simultaneously in both. Foci which can be removed without undue risk should be so treated as they develop, provided always that one sound kidney exists. Meanwhile the resistance of the organism to the bacillus is increased by proper living and by the injection of tuberculin. The mutilation does not seriously handicap the individual. 
One kidney suffices. He will probably be sterile whether epididymectomy is done or not, as the exudate plugs the channels of semination. If properly treated he should retain his testicles, as orchidectomy is necessary only in a very small percentage of cases.

Barney, J. D.: The Ultimate Results of Cienital Tuberculosis in the Male, Jour. Amer. Med. Assoc, 1914, 1xiii, 2274-2276.

CABot, H., and Barney, J. D.: Operative Treatment of (ienital Tuberculosis. Indications and Technic, Jour. Amer. NIed. Assoc., 1913, lxi, 2056-2058.

Dock, G., Young, E. L., Jr., Lower, W. E., Shlpe, T. P., and KretschMER, H. L.: Symposium on Tuberculosis of the Kidney, Trans. Amer. Urolog. Assoc., 1916, x, 199-261.

Lower, W. E., and Shupe, T. I'.: End-results of Nephrectomy for Renal Tuberculosis, Surg., Gynec., and Obst., 1917, xxv, 522-525.

Lyoss, O.: Tuberculosis of the (ienital Organs in Children, Jour. Amer. Med. Assoc., 1913, lxi, 2051-2054. 


\section{CHAPTER XV}

\section{GONOCOCCUS INFECTION IN THE FEMALE}

Vulvovaginitis of Little Girls. - The mucous membrane of the vagina of female children must be peculiarly susceptible to the gonococcus, judging from the ease with which this organism is transmitted. Among the children of well-to-do parents the disease is rare, although it may appear most unexpectedly. Under such circumstances the infection is probably transmitted by the nurse, or by someone, herself infected, who takes partial care of the child. In poor families, where living conditions are bad, the disease is often caught by the child's sleeping with an older girl who has gonorrhea. Direct infection undoubtedly does occur, either through intercourse with small boys or by contact with an older person. Epidemics of gonococcus vulvovaginitis in institutions are well recognized, and are due to dissemination of the infection through the medium of infected hands, infected towels or diapers, or infected bath-tubs.

The infection usually has an acute onset. Dammed back by the hymen, the discharge keeps the vaginal mucosa soaked with infectious material. The surplus discharge pours out from the vagina, and if ulcerative processes exist, it is streaked with blood. 
In the chronic stage the introitus is reddened and the clothes are stained by the thin, yellowish discharge; unless vigorously treated this condition exists for years. Rarely the infection may invade the fallopian tubes, and give the symptoms of acute salpingitis.

The diagnosis depends upon finding the biscuit-shaped Gram-negative diplococcus in pus-cells. In acute cases the gonococcus should be numerous and in almost pure culture. In cases of more than several weeks' standing the discharge may contain so many other bacteria that the gonococcus is difficult to find. The question has been raised whether the Gram-negative diplococci found in children who have but little discharge is really the gonococcus. It is true that there occur in little girls infections of the vagina which are not due to the diplococcus of Neisser. In our opinion the combination of purulent vaginal discharge and intracellular Gram-negative diplococci of typical morphology and arrangement means gonorrhea. In cases in which, when seen for the first time, the discharge is thin and scanty, the diagnosis is more difficult. The gonococcus, if it was the original infecting agent, is likely to be scarce. Such cases one should hesitate to diagnose as gonorrheal, particularly if there is no history of profuse purulent secretion, and no evident source of contamination.

The smear is best made by wrapping cotton about the tip of a probe, passing it into the depths of the vagina, and twisting it around. If the endoscope can be used, the smear should be taken through that. 


\section{AN OUTLINE OF GENITO-URINARY SURGERY}

The treatment in the acute stage must be soothing and cleansing. At first these children resist. If handled gently but firmly they quickly learn to behave and to allow the mother to give the treatments. In the very acute stage the flushing out of the vagina with 5 per cent. argyrol injected with a rubber ear syringe should be done three times a day. After each instillation a pledget of cotton, which quickly becomes soaked with the argyrol, is placed between the labia and the child is kept lying down for ten to fifteen minutes. After a week or so the instillation of argyrol should be preceded, at least once a day, preferably twice, by irrigation with a quart of warm potassium permanganate solution, about $1: 6000$ in strength. This is best given through a softrubber catheter inserted into the depths of the vagina; after the potassium permanganate has run out of the vagina the argyrol should be injected. A 10 per cent. solution may now be used.

This treatment must be kept up faithfully until no more discharge is seen. The argyrol may then be omitted and a daily douche of silver nitrate-1:6000-substituted. In some cases this will resurrect the discharge, and should be given up for the potassium permanganate douche. When the process appears to be cured, all treatment should be stopped and the child seen once a week for a month, and once a month for three months after that. At every visit smears made by introducing a dry cotton-tipped applicator into the depths of the vagina, there twisting it around, and then wiping it on a glass slide, should be examined for the presence of pus-cells. 
Pus-cells mean infection, and if they are present, the treatment should again be started. The cervix, even in very young girls, has been shown to be a stronghold of the gonococcus. In chronic cases the application of 10 per cent. silver nitrate to the walls of the vagina and to the cervix should be made through an electrically lighted straight urethroscope or endoscope.

The urethra must not be overlooked, as a urethritis may be the focus from which infection recurs. For that, urethral instillations of argyrol are given with a medicine-dropper. In my experience the accessory glands in girls under twelve are never infected.

If the above treatment is conscientiously followed cure can be achieved in the great majority of cases. Occasionally a very obstinate case seems to resist all efforts. Cultures of the vaginal secretion may show a predominance of some particular organism, and vaccine from that or from the gonococcus may be tried. The direct application of silver, however, seems to be the best single remedy for such cases.

One meets not infrequently the statement that vulvovaginitis in little girls is incurable, hence should be left alone. The same advice as to treatment is given by those who say that the infection will not persist beyond puberty.

With both these opinions we heartily disagree. Our experience at the Massachusetts General Hospital has shown that a proved cure may be expected in considerably over half of the patients treated, even when treatment is largely dependent upon the mother's co-operation. Probable cure, 


\section{AN OUTLINE OF GENITO-URINARY SURGERY}

with the disappearance of the gonococcus, can be obtained in practically all cases who will submit to the lengthy and tiresome measures which may be needed.

In the adult the gonococcus may invade the urethra, the glands of Skene and Bartholini, the glands lining the cervical canal, and the fallopian tubes. These are the points upon which treatment should be concentrated. In the acute cases douches twice a day of hot potassium permanganate $(1: 3000)$, the introduction of argyrol suppositories into the vagina, and the ingestion of capsules of sandalwood oil will quiet the infection. Local treatment consists of the instillation of argyrol ( 5 to 10 per cent.) into the urethra, the application to the cervical canal of tincture of iodin, silver nitrate (10 per cent.), or crude carbolic acid.

If the infection persists in the cervical glands, the application of the actual cautery to the walls of the cervical canal may be resorted to. Gonococci perish at $119^{\circ} \mathrm{F}$. This degree of heat can be reached without seriously injuring the tissues of this region.

If Skene's glands are involved, they must be injected with silver nitrate ( 1 per cent.) through a blunt-pointed needle and hypodermic syringe. If Bartholini's glands become infected, excision is the only cure. Otherwise the infection persists for years, not infrequently forming abscesses from time to time, and, at any rate, remaining a focus of reinfection.

Infection of the fallopian tubes must be treated symptomatically. For acute infection, rest in bed and hot douches 
twice daily should be tried. As a rule, the process will subside. If the signs of tubal inflammation entirely disappear, well and good. If the tube continues to be a thickened, infectious structure, keeping up a vaginal discharge or causing pelvic symptoms, salpingectomy should be considered. The more severe cases of pelvic infection must be seen by the surgeon, and, if pelvic peritonitis exists, the abdomen should be opened and the focus removed.

Johrson, W. S.: The Diagnosis of Latent Gonorrhea in the Female, Calif. State Jour. Med., September, 1912.

S.mith, G. G.: The Treatment of Gonococcus Vulvovaginitis, Amer. Jour. Dis. Children, 1914, vii, 230-237. 


\section{CHAPTER XVI}

\section{IMPOTENCE AND STERILITY}

DISORDERS of the sexual function in men deserve a book by themselves. Many pages could be filled with a description of their various manifestations and the reasons for their existence. The usual signs of loss of sexual power have to do with erection and ejaculation. Erection may be absent or illsustained. Ejaculation may be premature-that is, occurring before intromission or immediately thereupon. Secondary symptoms of sexual derangement include perineal or testicular ache following intercourse, backache, occipital headache, and generally "caved-in" feeling. According to Townsend and Valentine, impotence or sexual disorders are present in 6.5 per cent. of all genito-urinary cases.

"Sexual neurasthenics," so called, may be divided into two classes. The symptoms of Class One are due to psychic or purely functional causes. Examples of this type are the youth who fears to get married because he has masturbated in the past, and now, after long unsatisfied sexual excitement, because he finds a gluey urethral discharge, he believes he has "lost his manhood"; the man who has practised coitus interruptus until the sexual act is followed by prostatic pain and a feeling of exhaustion; the sexualist, who, after long- 
continued excessive indulgence, finds his powers growing less. In such cases regulation of sexual hygiene will effect a cure.

Class Two consists of those whose sexual symptoms are due to an actual, demonstrable pathologic condition. The line between these two classes is somewhat indefinite; the status of borderline cases must be determined by therapeutic test. If, following a correction of faulty habits alone, the symptoms are relieved, the case may fairly be put in Class One. There are others who show no definite abnormalities, who nevertheless do not respond to hygienic measures alone, but who require local treatment for relief of their symptoms. It is probable that every case of this sort shows some departure from the normal-undue redness of the verumontanum or a streaked appearance in the posterior urethra. If no definite pathology, such, for example, as infection of prostate or vesicles or papilloma of the posterior urethra, can be demonstrated, these cases should be classified with the functional disorders.

A considerable majority of all cases of sexual disorders take origin in physical abnormalities. Townsend and Valentine, from a study of 111 cases, conclude that 64 per cent. of sexual disorders are due to or originate in peripheral nerve irritation emanating from the posterior urethra or the colliculus seminalis. They found pathologic processes in the prostate in 86 , in the seminal vesicles in 45 , in the colliculus in 11 , lithemia in 15 , diabetes in 3 , intestinal auto-intoxication in 4 , disturbances of the kidney in 27 .

The treatment of those cases whose symptoms are due to 
psychic or functional causes consists, first of all, in getting the patient's point of view. The facts will probably have to be drawn out by sympathetic cross-questioning; during this process the physician should find out how much the patient knows about sexual matters, and how large a part of his trouble is due to ignorance or to psychologic factors. His fears may be founded upon perfectly natural phenomena. For example, many of my patients have mentioned as a sign of trouble the glycerin-like discharge which is the natural concomitant of long-continued sexual excitement. Some individuals cannot become aroused sexually unless the woman is of a certain type. The sexual libido is a queer appetite, capricious in some, in others omnivorous.

After hearing the patient out, one should examine his reflexes and external genitals, and should determine by rectal palpation the condition of the prostate and vesicles. This should be done in every case, even when no lesion is suspected. It goes far toward establishing the patient's confidence by giving the doctor's opinion a basis in fact. The urine should be examined for sugar; impotence is frequent in diabetics, and disappears when the urine becomes sugar free.

If no physical cause is found to account for the patient's . symptoms, he should be given a simple talk on sex physiology and set straight in his sexual hygiene. If these measures seem inadequate, a more thorough search for physical causes must be made. The secretion of the prostate and vesicles is examined for signs of inflammation. Possibly posterior urethroscopy should be done. It has been my experience 
never to have found, by urethroscopy, lesions which would not have been taken care of by the ordinary methods of treatment, such as dilatation, instillation of silver nitrate, and massage. Observers of much greater experience report various pathologic processes and advise correspondingly complex measures for their cure. It is my belief that at least 99 per cent. of all cases respond in a satisfactory manner to the simpler therapeutic measures.

The posterior urethra may show areas of congestion or granulation, perhaps bullous edema or cysts. The verumontanum is likely to be deeply suffused. These lesions, however, are usually the result of an underlying infection, and treatment of them, while it may temporarily relieve, will not eradicate the source of the trouble.

Even if no abnormalities are discovered, the sexual neurasthenic will be greatly benefited by dilatation of the posterior urethra with sounds or Kollmann dilator, instillations of 1 c.c. of 10 per cent. silver nitrate into the posterior urethra, and by gentle massage. Treatment should be given once a week.

If there is found to be urethritis or infection of the prostate or vesicles, some one of these three measures, or a combination of them all, must be relied upon to clear up both the actual lesion and the symptoms resulting therefrom.

Impotence Due to Seminal Vesiculitis.-J. A. B. was an example of this. He was a postal clerk, age thirty-eight, married, six children. He came for advice partly because of loss of sexual vigor, accompanied by emissions, partly because he had been told he had Bright's disease. He had never had any venereal disease or any serious illness. For the preceding year he had had dull pain in both flanks, and had noticed a change in his sexual powers. 


\section{AN OUTLINE OF GENITO-URINARY SURGERY}

Physical examination of chest and abdomen was negative. Systolic blood-pressure 158. External genitalia were normal. No stricture. Right lobe of prostate and seminal vesicle were matted together and gave a feeling of soft distention. Secretion on massage showed a large amount of pus. Urine: Clear with a few shreds; specific gravity 1012; albumin slightest trace; sugar 0. Sediment: A few leukocytes and epithelial cells; no casts. Cystoscopy showed a normal bladder, save for some redness of trigone. Prostatic middle lobe shows some enlargement. Both ureters appear normal and emit clear jets. $x$-Ray showed no stones.

He was treated for two or three months by dilatation of the posterior urethra with the Kollmann dilator, silver nitrate irrigations, and massage. As the condition of his vesicles improved, as judged by the evidence of the palpating finger, his sexual powers increased and his pain diminished. When last seen he had no complaint as regards his sexual function. The vesicles were much softer and the exudate nearly absorbed. His backache continued, and was said by an orthopedist to be caused by pronation of feet.

In the management of these cases the general condition of the patient must be considered. Change of scene, recreation, open air, will often do much more than local treatment. Sexual intercourse must be stopped during active treatment and alcohol is forbidden.

Sterility.-The problem of sterility is, as a rule, entirely separate from that of impotence. The woman's failure to conceive may be the result of abnormalities of the female organs of generation, or may be due to the husband's inability to impregnate. Rarely the difficulty resides in the reaction between the male and female secretions, either individual, with a different partner, being fertile. It goes without saying that a union in which the semen cannot be deposited in the vagina will be sterile. Obstacles to copulation are: in the female, excessive obesity, absence of vagina, imperforate hymen, and vaginismus; in the male, impotence, 
well-marked epispadias or hypospadias, exstrophy of the bladder.

Granting for the moment that the husband is normal, there are various conditions in the wife which prevent conception. ${ }^{1}$ These may be divided into congenital abnormalities, acquired abnormalities, results of inflammation, and functional disability.

Congenital abnormalities include absence or atresia of the vagina, double or septate vagina, smallness of the cervical orifice, infantile uterus (frequently accompanied by underdevelopment of the ovaries).

Acquired abnormalities of non-inflammatory nature which may prevent conception are uterine polyp, fibroid tumors of the uterus, and ovarian and parovarian cysts. Lacerations of perineum and of cervix and retroversion are not considered by Kelly to be frequent causes of sterility.

Inflammatory conditions which are hostile to and perhaps prohibitive of conception are caused to a very great extent by the gonococcus. The change in reaction from alkaline to acid which the products of infection may bring about in the cervical secretion is hostile to the spermatozoa. The formation of abnormally thick, viscid secretion may prevent their passage through the cervical canal. Infection of the tubes is a frequent cause of infertility. During the stage of pus formation the spermatozoa are likely to be killed by the acid, enzymotic secretion; later the lumen of the tube may

${ }^{1}$ For an excellent presentation of this aspect of the question, see Kelly, "Medical Gynecology," 1909, pp. 339-356. 


\section{AN OUTLINE OF GENITO-URINARY SURGERY}

be strictured and impervious. Tuberculous salpingitis was found by Sänger to be the cause of sterility in 1 out of 397 childless marriages.

The functional causes of sterility are less definite and are more difficult of diagnosis. Excessive obesity, probably through alterations in the secretions of the thyroid, adrenals, and ovaries, is considered by Kelly to be a possible cause. Alcoholism and morphinism affect the sexual cycle in women. Acute infectious diseases may cause atrophy of the ovaries, just as mumps may cause atrophy of the testicle in man. Unless some definite abnormality is suggested by the history or the physical examination, one is not justified in attributing sterility to the condition of the ovary until every other possible cause has been excluded.

On the masculine side, sterility may be due to factors, mentioned above, which prevent the deposition of semen within the vagina. Stricture of the urethra and polyp of the posterior urethra may prevent the discharge of the seminal fluid in sufficient quantities to impregnate. Occlusion of the ejaculatory ducts gives rise to the condition known as "aspermia."

As regards the semen itself, it may contain no spermatozoa (azoöspermia), or only a few (oligospermia), or those which are present may be dead or lacking in vitality (necrospermia). The cause of azoöspermia and oligospermia may reside in the testicle itself. Certain diseases of the testis, such as the orchitis of mumps and perhaps that of typhoid fever, result in atrophy of the spermatogenetic cells. Cryptorchidism, 
as a rule, does the same thing, although cases have been reported in which normal spermatozoa were secreted by undescended testes. Excessive obesity has been mentioned as being a possible cause of azoöspermia, and sexual excess will, temporarily at least, cause a disappearance of spermatozoa from the semen. Occlusion of the vas or of the epididymal tube will, of course, prevent the passage of spermatozoa. If the process is bilateral, sterility results. This is seen in tuberculous epididymitis, sometimes when only one epididymis is involved. It is then probably due to the invasion of both seminal vesicles by the process. Gonorrheal epididymitis is a frequent cause of sterility. Out of 242 cases of double epididymitis, Finger found 207 cases of azoöspermia.

Inflammatory processes in the seminal vesicles may block the outlets. A much more usual effect, however, is the production of necrospermia. The spermatozoa are weakened or killed by the acid products of suppuration. Although the gonococcus is responsible for the vast majority of such cases, it must be borne in mind that infection by other organisms may occur in men who have never had gonorrhea.

Brief reflection upon the foregoing facts will speedily bring one to the conclusion that the gonococcus is responsible for sterility in a large percentage of all cases. From a study of sterility statistics in both men and women it would seem that at least half of the cases might be attributed to this etiologic factor. Another fact which impresses itself upon one is the large number of cases in which responsibility for an unfruitful union rests with the man. Sänger, in analyzing 
110 childless marriages, found that in 45.4 per cent. the husband's spermatozoa were absent or greatly reduced in number. Kehrer, in a series of 96 such marriages, found the husband sterile in 36 per cent. Gross, in 192 cases, blamed the man in 18 per cent. More figures could be set forth; these are enough to prove that in every case of sterility the condition of the husband as well as that of the wife should be thoroughly investigated.

The study of such a case requires first a careful history of the sexual life of both individuals. The wife should be examined for gross abnormalities of the generative organs. The semen of the husband should then be examined. After at least a week of continence his discharge should be collected in a condom, which should be suspended in a widemouthed bottle of tepid water and brought at once for inspection. The quantity and reaction of the seminal fluid should be noted. The normal reaction is alkaline to litmus; acidity is the result of inflammation of the vesicles. A few drops are placed upon a warm slide and examined under the high dry power of the miscroscope. In normal semen the number of spermatozoa is tremendous. Lode estimated that there were $200,000,000$ in one ejaculation. Yet of these comparatively few reach the cervical canal. The reaction of the vaginal secretion is normally faintly acid, and is not conducive to long life on the part of the spermatozoa. It is easy to see, therefore, that with semen containing only a few spermatozoa the chances of impregnation are very poor. The spermatozoa should be uniform in size and shape, and a 
fair proportion of them should be vigorously motile even after several hours. Reynolds divides the life of a spermatozoön after ejaculation into three periods: (1) The "progressive vibratile," characterized by a violent side-to-side lashing of the tail which drives the spe $m$ straight ahead, always against the current. (2) The "undulatory tactile," in which the tail makes long, slow sweeps and the head weaves from side to side. During this phase the spermatozoön seems to nose its way among the cells and débris of the secretion. (3) The "stationary bunting," during which the sperm endeavors to force its way into the cell with which it is in contact. Of these three phases, the last is never seen under artificial conditions. Persistence of the first stage is an indication of strong vitality; in weak specimens the second stage appears early.

If healthy spermatozoa do not appear in a specimen of semen, the observation should be repeated at least three times. If the semen is always abnormal, the cause for this abnormality must be sought. Azoöspermia suggests bilateral disease of the testicles or blockage of the seminal canal. Oligo- or necrospermia suggests inflammatory disease of the seminal vesicles. If the patient has had bilateral epididymitis, and now has no spermatozoa in his semen, the operation of vaso-epididymostomy, devised by Martin, may be done. This consists of anastomosing the vas deferens, which must be proved to be patent below the point of anastomosis, to the upper pole of the epididymis. In doing this operation upon 18 patients Wolbarst met with definite success in but 1 . 
Martin reported 7 cases. In 4 spermatozoa appeared in the semen, 2 were not traced, and 1 was a failure. Lespinasse has modified Martin's operation, and reports success in 10 out of 11 operations performed on dogs.

For the condition of oligo- or necrospermia caused by inflammation of the vesicles a long course of massage and dilatation must be undertaken in the hope that the exudate will absorb and the infection die out or become avirulent. Possibly drainage of the vesicles might be valuable.

If the semen obtained by condom is normal, and the wife shows no gross abnormalities, specimens of cervical secretion should be obtained at varying periods after coitus in order that the effect of the cervical secretion upon the spermatozoa may be studied. Death of the male element when mixed with cervical secret:on may be due to the products of infection, or to intolerance of the blood-serum of the woman for the spermatozoa of the man. If to the former, treatment must be given for eradication of the infection. If an unusually acid reaction exists in the vaginal secretion, douches of sodium bicarbonate taken just before coitus may be advised.

This is about as far in the treatment of sterility as the physician who has not specialized in this subject can go.

Further measures consist in securing specimens of uterine secretion from the fundus in order to determine their effect upon the sperm. If they are not incompatible, and the cervical secretion is hostile, artificial impregnation may be tried, a few drops of semen being injected into the cavity of the uterus. This procedure may also be tried when the man, 
because of some deformity, such as hypospadias, is unable to deposit semen within the vagina.

The value of glandular therapy in certain cases of sterility has not been thoroughly worked out. Cases have been reported in which conception has followed the administration of glandular extract, directed toward stimulation sometimes of the ovaries, sometimes of the testes, depending upon whether the male or the female is at fault. This should be tried only when all anatomic lesions are ruled out.

There are many cases of sterility in which the cause is easy to determine. There are others which baffle even those who have gone most deeply into this fascinating study. Much progress has been made in recent years, and, as interest in the problem grows, more can be expected. One lesson at least should be thoroughly learned. That is, that operative measures upon the wife for the relief of sterility should never be undertaken until the fact of the husband's fertility has been thoroughly established.

BarNey, J. D.: Observations on Sterility in the Male, Boston Med. and Surg. Jour., 1914, clxx, 943-947.

Kol., I. S.: Etiology, Pathology, and Treatment of Sexual Impotence, Urologic and Cutaneous Rev., 1915, xix, 541-544.

Lespinasse, V. D.: Obstructive Sterility in the Male. Treatment by Direct Vaso-epididymostomy, Jour. Amer. Med. Assoc., 1918, lxx, 448-450.

Reynolds, E.: Fertility and Sterility, Jour. Amer. Med. Assoc., 1916, Ixvii, 1193.

Townsend, T. M., and Valentine, J. J.: Functional Sexual Disorders Proceeding from the Genito-urinary Tract, Med. Record, May 27, 1911.

Wolbarst, A. L.: Surgical Aspects of Male Sterility, New York Med. Jour., May 19 and 26, 1917. 



\section{INDEX}

Abdominal tumor associated with hematuria, 224

of renal origin, 220

of splenic origin, 223

Aberrant vessels in hydronephrosis, 243

Abnormalities of spermatozoa, 284 of urine in renal disease, 216

Abortion of gonorrhea, 74

Abscess of prostate, 108 of testicle, 181

perineal, from seminal vesicle, 110

Acid, boric, in bladder lavage, 19

Acute cystitis, 191

hematogenous kidney, 234

Adenitis, inguinal, 61

Adenomatous prostate, 124

Albumin, tests for, 40

Alexander bandage, 171

Alkaline cystitis, 195

Anatomy of seminal vesicles, 109

Anesthesia in cystoscopy, 28, 262

in prostatectomy, 147

local, for circumcision, 67

for cystotomy, 138

for operations on scrotum, 168

spinal, in cystoscopy, 29

in perineal section, 102

technic, 147

Antiseptics, urinary, action of, 33 administration before instrumentation, 20
Argyrol in urethritis, 79, 276

in vulvovaginitis, 274

Arthritis, gonococcus, 111

vaccines in, 112

Asepsis in urethral instrumentation, 20

Bacillus acidophilus in alkaline cystitis, 95

colon, epididymitis due to, 185

infection of kidney, 234

Ducrey's, 61

tubercle, detection by inoculation of guinea-pig, 41

in urine, 41

Bacteria in urinary sediment, 41

Bag, Hagner, 149

Balanitis, 64

Balsamics, action of, 33

Bandage, Alexander, 171

Bartholini's glands, infection by gonococcus, 276

Bilateral nephrostomy in cancer of bladder, 210

ureterostomy in cancer of bladder, 210

Bilharziasis, 203

Bladder, Bilharzia infection of; 203 cancer of, 204

bilateral nephrostomy in, 210

ureterostomy in, 210

radium in, 212

diseases of, 188 
Bladder disturbances due to multiple sclerosis, 200

to nerve lesions, 200

to spina bifida, 200, 203

to syringomyelia, 200

diverticulum of, 50

$x$-ray of, 52

encrusted, 195

examination of, 29

exstrophy of, 54

infections of, 189

lavage of, 19

in cystitis, 193

neck, contracture of, 157

overflow, 126

paracentesis of, 142, 144

paralysis due to diphtheria, 200 to tabes, 198

to transverse myelitis, 200

rupture of, 213

stone in, 212

after prostatectomy, 156

syphilis of, 203

tabetic, 199

tuberculosis of, 261

treatment of, 264

tumor of, 204

removal of, 207

thermocoagulation of, 206

Boric acid in bladder lavage, 19

Brown-Buerger cystoscope, 28

Bubo, 61, 62

Burnam's test for formalin in urine, 37

Calculi, oxalate, 253

phosphatic, 254

renal and ureteral, 245, 247

recurrent, 253

$x$-ray of, 247, 254

vesical, 212

Cancer of bladder, 204
Cancer of bladder, bilateral nephrostomy in, 210

ureterostomy in, 210

radium in, 212

of penis, 71

of prostate, 158, 161

differential diagnosis of, 130

radium in, 160

Caruncle of urethra, 105

Case reports:

bladder, encrusted, 195

tumor removed by fulguration, 207

by operation, 207

decapsulation for renal hematuria, 227

diverticulum of bladder, 52

epididymitis, tuberculous, 265

hematuria, essential, 218

of chronic nephritis, 217

hydronephrosis, 243

hypernephroma, 221

impotence due to seminal vesiculitis, 281

kidney, rupture of, 258

multiple sclerosis cause of bladder disturbance, 201

mumps, epididymo-orchitis of, 179

nephrectomy for tuberculosis, 269

nephropexy, 231

prostate, cancer of, 161

prostatectomy, perineal, 154

suprapubic, 154

prostatic enlargement, first stage, 126

pyelitis, chronic bilateral, 241

of pregnancy, 236

renal and ureteral stone, recurrent, 254 
Case reports:

renal stone, hematuria the only symptom, 247

seminal vesicle, perineal abscess from, 110

vesiculitis, chronic, 117

non-specific, 118

syringomyelia, bladder dis-

turbance due to, 200

tabes dorsalis, retention due to, 131

testicle, abscess of, 181

tumor, abdominal, of splenic origin, 223

ureterolithotomy, 251

urethritis, acute, and stricture, 86

chronic, 92, 93, 94

in female, 105

non-specific, 91

Catheter life for prostatics, 135

lubricant, 20, 23

method of fastening in urethra,

144

specimen in examination of urine,

39

Catheterization in prostatic ob-

struction, 142

of ureters, 31

retrograde, 100

Cervix uteri, infection of, by gonococcus, 276

Chancre, 62

Chancroid, 61

Children, cystoscopy in, 31

pyelitis in, 237

Chronic cystitis, 196 urethritis, 87

Circumcision, indications for, 65 local anesthesia for, 67 technic of, 65

Coccus infection of kidney, 234

Colic, renal and ureteral, 219 treatment, 253

Colon bacillus epididymitis, 185

infection of kidney, 234

Complement-fixation test in gonorrhea, 43

technic of, 43

value in chronic urethritis, 93

Condylomata, 63

due to syphilis, 64

Congenital malformations of genitourinary tract, 49

Contracture of bladder neck, 157

Culture of urine, 41

Cure of gonococcus infections, 120

Cyst, echinococcus, of kidney, 257 of urachus, 54

Cystectomy, 209

Cystitis, 189

acute, 191

alkaline, Bacillus acidophilus in, 195

chronic, 196

causes of, 197

lavage of bladder in, 193

Cystoscope, Brown-Buerger, 28

operating, 31

Cystoscopy, anesthesia in, 28, 262

spinal, 29

in children, 31

indigo-carmine in, 30 .

operative, 31

technic of, 28

value of, 27

Cystotomy in two-stage prostatectomy, 138

local anesthesia for, 138

Cysto-urethroscope in posterior urethra, 26

Decapsulation in nephritis, 228

in renal hematuria, 227 
Diet in lithiasis, 253

Dietl's crisis, 230

Dilatation of stricture, 98

Diphtheria a cause of bladder paralysis, 200

Diverticulum of bladder, 50 $x$-ray in, 52

Double pelvis, 49 ureter, 49

Ducrey's bacillus, 61

Dystopic kidney, 49, 50

Echinococcus cyst of kidney, 257

Edebohl's operation, 228

Embryoma of kidney, 255

Fncrusted bladder, 195

Endoscopy in female, 25, 26, 104 technic of, 25 value of, 24

Epididymis, gonococcus infection of, 185

tuberculosis of, 265

Epididymitis, 183 acute, treatment of, 186 due to colon bacillus, 185

Epididymo-orchitis of mumps, 178, 179

Epididymotomy, 186

Epididymovasectomy, 267

Epispadias, 55

Equipment for minor genito-urinary surgery, 22

Essential hematuria, 226

Exstrophy of bladder, 54

External urethrotomy, 99

Extravasation of urine, 101

FaLlopian tubes, infection by gonococcus, 276

Fehling's test for sugar, 40

Female, endoscopy in, 25, 104 gonococcus infection in, 272
Fever in renal infection, 220

Fistula of urachus, 54

Formaldehyd sterilizer, 21

Formalin in urine, test for, 37

Fulguration, removal of bladder tumor by, 207

Function, renal, tests of, 44, 45

Genital tuberculosis, 265

Genito-urinary surgery, minor, equipment for, 22

technic, 18

tract, congenital malformations of, 49

tuberculosis, 260

Gonococci in secretion from prostate and vesicles, 42

morphology of, 42

Gonococcus, arthritis due to, 111 vaccines in, 112

cause of sterility, 285

epididymitis, 185

infection, cure of, 120

of Bartholini's glands, 276

of cervix uteri, 276

of female, 272

of prostate, 77,85

of Skene's glands, 276

septiceniid, 111

vulvovaginitis, 272

Gonorrhea, abortion of, 74

acute, treatment of, 77

and marriage, 122

complement-fixation test in, 43

cure of, 84

- test for, 121

in female, 272

in male, 73

prevention of, 73

Gram's stain, 42

Guinea-pig, inoculation of, detec-

tion of tubercle bacillus by, 41 
HAGNer bag, 149

Hegonon in urethritis, 79

Hematocele, 164

Hematuria associated with abdominal tumor, 224

essential, 226

of chronic nephritis, 217

Hermaphroditism, 56

Hexamethylenamin, action of, 34

Horseshoe kidney, 49

Hydrocele, 163

of cord, 174

radical cure of, 168

tapping of, 165

Hydronephrosis, 225, 243

Hygiene, sex, in impotence, 280

Hypernephroma, 221, 255

Hypertrophy of prostate, 124

Hypospadias, 56

\section{IMPOTENCE, 278}

due to seminal vesiculitis, 281

rôle of posterior urethra in, 281

sex hygiene in, 280

Incontinence, urinary, in women, 106

Indigo-carmine in cystoscopy, 30

Infection, acute, of prostate, 107 of seminal vesicles, 107

Bilharzia, of bladder, 203

chronic, of prostate, 91

due to trauma, 19

gonococcus, cure of, 120

of female, 272

of prostate, 77,85

of bladder, 189

of cervix uteri by gonococcus, 276 of fallopian tubes by gonococcus, 276

of kidney, 232, 234

coccus, 234
Infection of Skene's glands, 103, 276

of vesicles, 77,85

renal, fever in, 220

vaccines in, 240

urinary, salol in, 38

Inguinal adenitis, 61

Instruments, sterilization of, 20

Insufficiency, renal, 225

Internal urethrotomy, 98

Irrigator, use in urethritis, 83

KEYES' instillator, 25, 120

Kidney, acute hematogenous, 234

colon bacillus infection of, 234

cysts of, 257

diseases of, 216

dystopic, 49, 50

embryoma of, 255

function, tests of, 44

horseshoe, 49

infections of, 232, 234

movable, 228

polycystic, 225,257

rupture of, 257, 258

single, 49

stone in, 245

tuberculosis of, 261

tumor of, 255

pyelography in diagnosis of, 221

Weigert, 226

LACTIC acid bacilli in alkaline cystitis, 195

Lavage in pyelitis, 236, 240

of bladder and urethra, 19

in cystitis, 193

of renal pelvis, 236, 240

Lithiasis, diet in, 253

Lubricant, catheter, 20, 23 
MALE, gonorrhea in, 73

Malformations, congenital, of genito-urinary tract, 49 of penis, 55

Massage of prostate and vesicles, 116,119

Maydl operation, 55

Meatotomy, 88

Morphology of gonococci, 42

Movable kidney, 228

Multiple sclerosis cause of bladder disturbances, 200, 201

Mumps, epididymo-orchitis of, 178 , 179

Myelitis, transverse, cause of bladder paralysis, 200

NEck of bladder, contracture of, 157

Neoplasm of testicle, 183

Nephrectomy for tuberculosis, 262, 269

tuberculin after, 263

Nephritis, decapsulation in, 228 hematuria in, 217

Nephropexy, 230, 231

Nephroptosis, 228

Nephrostomy, bilateral, in cancer of bladder, 210

Nerve lesions cause of bladder disturbances, 200

Neurasthenia, sexual, 278

OBSTRUCTION, prostatic, 124 catheterization in, 142 retention due to, 136

Oil, sandalwood, action of, 33 in urethritis, 78

Otis urethrotome, 98

Oxalate calculi, 253
PaIN, renal, 219

Paracentesis of bladder, 142, 144

Paralysis of bladder due to diphtheria, 200 to tabes, 198

Paraphimosis, 70

Pelvis, double, 49 renal, lavage in, 236, 240

Penis, cancer of, 71 diseases of, 61 malformations of, 55

Perineal abscess from seminal vesicle, 110 prostatectomy, 154, 161 prostatotomy, 157 section for stricture of urethra, 99 spinal anesthesia in, 102

Phenolsulphonephthalein test of renal function, 45

Phimosis, 64

Phosphatic calculi, 254

Phthalein test, technic of, 45

Polycystic kidney, 225, 257

Potassium citrate in urethritis, 78 permanganate in bladder lavage, 19

in urethritis, 78

tablets of, 23

Pregnancy, pyelitis of, 235, 236

Prepuce, tightness of, 64

Prolapse of urethra, 106

Prophylaxis, venereal, 73

Prostate, abscess of, 108 acute infections of, 107 adenomatous, 124 benign enlargement of, 124 cancer of, 158, 161 differential diagnosis, 130 radium in, 160 chronic infection of, 91 diseases of, 107 gonococcus infection of, 77, 85 
Prostate, hypertrophy of, 124

massage of, 116, 119

secretion from, gonococci in, 42

small fibrous, 157

tuberculosis of, 123, 268

Prostatectomy, 137

after-treatment of, 156

anesthesia in, 147

spinal, 147

perineal, 15.3, 154, 161

postoperative treatment of, 155

stone in bladder after, 156

suprapubic, 145,154

two-stage, cystotomy in, 138

Prostatic enlargement, symptoms of, 125

obstruction, 124

catheterization in, 142

retention due to, 136

secretion, stain for, 43

Prostatics, catheter life for, 135 management of, 132

Prostatitis, chronic, 114 in stricture, 96

non-specific, 118

Prostatotomy, perineal, 157

lavage in, 236, 240

Punch, Young's, 157

Pyelitis, chronic bilateral, 241

of children, 237

of pregnancy, 235, 236

sequelae of, 238

silver nitrate in, 240

treatment of, 240

Pyelography in diagnosis of renal tumor, 221

Pyelonephritis, 234

Pyuria, symptoms of renal disease, 219

Radium in cancer of bladder, 212 of prostate, 160
Renal colic, 219, 253

disease, pyuria, symptom of, 219 urine abnormalities in, 216

function, tests for, 44,45

infections, 232

fever in, 220

vaccines in, 240

insufficiency, 225

pain, 219

pelvis, lavage of, 236, 240

retention, 243

stone, 245

bilateral, 248

hematuria, only symptom, 247

recurrent, 254

$x$-ray in, 247

tumor, 255

pyelography in diagnosis of, 221

Retention due to prostatic obstruction, 136

catheterization in, 142

to stricture, 97

to tabes dorsalis, 131

renal, 243

Retrograde catheterization, 100

Rupture of bladder, 213

of kidney, 257, 258

of urethra, 95

SALOL in urinary infections, 38

Salpingitis, treatment of, 276

Sandalwood oil, action of, 33

in urethritis, 78

Sclerosis, multiple, bladder disturbances due to, 200, 201

Scrotum, diseases of, 163

local anesthesia for operations on, 168

Sediment, urinary, examination of, 40

Semen, examination of, 284 
Seminal vesicles, acute infections of, 107 anatomy of, 109 diseases of, 107 massage of, 116, 119 medication of, 113 perineal abscess from, 110 tuberculosis of, 123, 268 vesiculitis, chronic, 114, 172 impotence due to, 281 non-specific, 118 vesiculotomy, 113

Septicemia, gonococcus, 111

Sex hygiene in impotence, 280

Sexual neurasthenia, 278.

Silver nitrate in bladder lavage, 19 in chronic urethritis, 89

in posterior urethritis, 27,120 in pyelitis, 240

tablets of, 23

Single kidney, 49 ureter, 49

Skene's glands, infection of, 103, 276

Sodium acid phosphate a means of acidifying urine, 36

benzoate in urinary infections, 38 bicarbonate douche in sterility, 288

Sounds in chronic urethritis, 88 in stricture of urethra, 98

Spermatic cord, diseases of, 174 hydrocele of, 174

Spermatocele, 163

Spermatozoa, abnormalities of, 284 stained, 43

Spina bifida cause of bladder disturbances, 200 occulta in bladder disturbances, 203

Spinal anesthesia in cystoscopy, 29 in perineal section, 102
Spinal anesthesia in prostatectomy, 147 technic, 148 Spirochæta pallidum, 62

Stain for prostatic secretion, 43 for tubercle bacillus, 41 for urethral secretion, 42 Gram's, for gonococci, 42

Stained spermatozoa, 43

Sterility, causes of, 282 gonococcus, 285 sodium bicarbonate douche in; 288

Sterilization of instruments, 20

Sterilizer, formaldehyd, 21

Stone in bladder, 212 after prostatectomy, 156 renal and ureteral, 245 recurrence of, 253, 254 $x$-ray of, 247

Stricture, dilatation of, 98 in acute urethritis, 86 in chronic urethritis, 87 in women, 105 of urethra, 95 perineal section for, 99 sounds in, 98 prostatitis in, 96 rules for treatment of, 102 tuberculous, 96 urethrotomy for, 98

Sugar, test for, 40 Suprapubic prostatectomy, 145,154 Syphilis, condylomata due to, 64 initial lesion of, 62 of bladder, 203 of testicle, 182 Syringomyelia cause of bladder disturbance, 200

TABES dorsalis cause of bladder paralysis, 198 
Tabes dorsalis, test for, 131

Tabetic bladder, management of, 199

Tapping of hydrocele, 165

Technic in genito-urinary surgery, 18 of circumcision, 65 of complement-fixation test, 43 of cystoscopy, 28 of endoscopy, 25

Teratoma of testicle, 183

Test, Burnam's, for formalin in urine, 37

complement-fixation, 43

for albumin, 40

for formalin in urine, 37

for sugar, 40

for tabes dorsalis, 131

of cure of gonorrhea, 121

of renal function, 44

phthalein, technic, 45

Testicle, abscess of, 181

diseases of, 178

neoplasm of, 183

syphilis of, 182

teratoma of, 183

torsion of, 176

trauma of, 178

tuberculosis of, 182

undescended, 57

Thermocoagulation of bladder tumors, 206

Torsion of spermatic cord, 176 of testicle, 176

Transverse myelitis cause of bladder paralysis, 200

Tubercle bacilli in urine, detection of, 41

stain for, 41

Tuberculin after nephrectomy, 263

Tuberculosis, genital, 265

genito-urinary, 260
Tuberculosis, nephrectomy for, 262, 269 of bladder, 261

treatment, 264

of epididymis, 265

of kidney, 261

of prostate, 123, 268

of testicle, 182

of vesicles, 123, 268

Tuberculous stricture, 96

Tumor, abdominal, of renal origin, 220

of bladder, 204

thermocoagulation of, 206

of kidney, 255

renal, 255

pyelography in diagnosis of, 221

UNDESCENDED testicle, 57

Urachus, cyst of, 54

fistula of, 54

Ureter, diseases of, 216

double, 49

single, 49

Ureteral colic, 219, 253

recurrent, 254

stone, 245

Ureterolithotomy, 251

Ureterosigmoidostomy, 55

Ureterostomy, bilateral, in cancer of bladder, 210

Ureters, catheterization of, 31

Urethra, anterior, examination of, 26

caruncle of, 105

diseases of, 73

in women, 103

female, endoscopy of, 25, 26, 104

lavage of, 19

method of fastening catheter in, 144 
Urethra, posterior, appearance of, Urinary incontinence in women, 27

cysto-urethroscope in, 26

rôle in impotence, 281

urethritis involving, 81

prolapse of, 106

rupture of, 95

stricture of, 95

in women, 105

perineal section for, 99

sounds in, 98

Urethral instrumentation, asepsis in, 20

secretion, stain for, 42

Urethritis, acute, and strictur , 86 in women, 103

treatment of, 77

argyrol in, 79, 276

chronic, 87

in women, 103, 105

silver nitrate in, 89

sounds in, 88

stricture in, 87

value of complement-fixation test in, 93

cure of, 89

hegonon in, 79

involving posterior urethra, 81

non-specific, 90, 91

posterior, silver nitrate in, 27 ,

120

potassium citrate in, 78

permanganate in, 78

use of irrigator in, 83

Urethrotome, Otis, 98

Urethrotomy, external, 99

for stricture, 98

internal, 98

Urinalysis, 39

Urinary antiseptics, action of, 33

administration before instrumentation, 20

106

infections, salol in, 38

sodium benzoate in, 38

sediment, bacteria in, $\mathbf{4 1}$

examination of, 40

Urination, disturbances of, 188, 220

Urine abnormalities in renal disease, 216

acidity increased by sodium acid phosphate, 36

culture of, 41

examination of, 39

extravasation of, 101

test for formalin in, 37

tubercle bacillus in, 41

Urotropin. See Hexamethylenamin, 35

VACCINES in gonococcus arthritis, 112

in renal infections, 240

Varicocele, 175

Vaso-epididymostomy, 287

Venereal prophylaxis, 73

warts, 63

Vesical calculus, 212

Vesicles, infection of, 77, 85 massage of, 116, 119

seminal, acute infections of, 107 massage of, 116, 119 perineal abscess from, 110 tuberculosis of, 123, 268

Vesicular secretion, gonococci in, 42 stain for, 43

Vesiculitis, seminal, chronic, 117 due to impotence, 281 non-specific, 118

Vesiculotomy, seminal, 113

Vulvovaginitis, 272

argyrol in, 274

gonococcus, 272 
WARTS, venereal, 63

Weigert kidney, 226

Women, diseases of urethra in, 103 urinary incontinence in, 106
X-ray of bladder diverticulum, 52 of renal stone, 247

Young's punch, 157 



\section{SAUNDERS' BOOKS}

\section{on \\ Skin, Genito-Urinary,}

\section{Dentistry, Chemistry, and Eye, Ear, Nose, and Throat}

W. B. SAUNDERS COMPANY

West Washington Square

Philadelphia

9. Henrietta Street

Covent Garden, London

Our Handsome Complete Catalogue will be Sent on Request

\section{Stelwagon on the Skin}

A Treatise on Diseases of the Skin. By Henry $W$. Stelwagon, M. D., Ph. D., Professor of Dermatology in the Jefferson Medical College, Philadelphia. Octavo of 1309 pages, with 356 text-cuts and 33 plates. Cloth, $\$ 6.5 \circ$ net.

Eighth Edition published November, 1916

The demand for eight editions of this work in such a short period indicates the practical character of the book. In this edition the articles on Frambesia, Oriental Sore, and other tropical diseases have been entirely rewritten. The new subjects include Occupational Dermatoses, Paraffinoma, Purpura Annularis, Telangiectodes, Xanthoma Elasticum, and Ulerythema Ophryogenes. George T. Elliot, M. D., Professor of Dermatology, Cornell University, says: "It is a book that I recommend to my class at Cornell, because for conservative judgment, for accurate observation, and for a thorough appreciation of the essential position of dermatology, I think it holds first place."

Our books are revised frequently, so that the editions you find here may not be the latest. Write us about any books in which you are interested 


\section{Schamberg's Diseases of the Skin and Eruptive Fevers}

Diseases of the Skin and Eruptive Fevers. By JAY F. Schamberg, M. D., Professor of Dermatology and the Infectious Eruptive Diseases, Philadelphia Polyclinic. Octavo of 585 pages, illustrated. Cloth, $\$ 3.25$ net.

\section{THIRD EDITION-published September, 1915}

Dr. Schamberg takes up all diseases of the skin, giving special emphasis to those diseases met most frequently in general practice. The work is particularly full on actinotherapy, rontgenotherapy, and radium, these modern measures being discussed in a separate chapter as well as under the various diseases. The exanthemata are considered in a special chapter, diagnosis and treatment being given unusual space. In addition, there are described the usual and the accidental eruptions occurring in such diseases as typhoid, epidemic cerebrospinal meningitis, influenza, malaria, tonsillitis, etc. This is an important feature. All the new vaccines and serums are considered-their use both in diagnosis and treatment. The many comparative tables of symptoms and the wealth of reliable prescriptions make "Schamberg" a most practical work for the general practitioner as well as for the specialist.

\section{Johns Hopkins Hospital Bulletin}

"The descriptions of the eruptions are so clear and concise that the appearance of a disease can readily be imagined. The arrangement of diagnosis of many of the diseases is excellent, the points considered being placed opposite one another in parallel rows."

\section{Asher's Chemistry \& Toxicology for Nurses}

Chemistry and Toxicology for Nurses. By Philip Asher, PH. G., M. D., Dean and Professor of Chemistry, New Orleans College of Pharmacy. I2mo of 190 pages. Cloth, $\$ 1.25$ net.

Dr. Asher's one aim in writing this book was to emphasize throughout the applica. tion of chemical and toxicologic knowledge in the practice of nursing. This he has succeeded in doing. The nurse, both in training-school and in graduate practice. will find it extremely helpful because the subject is made so clear. October, 1914 


\section{Koll's Diseases of Male Urethra}

Diseases of the Male Urethra. By Irvin S. Koll, M.D., Professor of Genito-urinary Diseases, Post-Graduate Hospital and Medical School, Chicago. Octavo of $\mathrm{I}_{50}$ pages, with many illustrations, some in colors. Cloth, $\$ 3.00$ net. Published June, 1918

\section{ALL DISEASES OF THE MALE URETHRA}

Gonorrheal urethritis is a condition that every physician, whether general practitioner or specialist, is called upon to treat frequently. Dr. Koll gives you here a clear, concise, and simple treatise, rendered additionally helpful by the extensive use of original illustrations taken from the author's own clinic. He gives full consideration of every aspect of gonorrhea - anatomy of the organs involved, bacteriology, pathology (here illustrated for the first time in any text), symptomatology, diagnosis, and treatment. The section on treatment goes so thoroughly into active, medicinal, and local therapy (including the use of albargin and astringents) that any practitioner can treat the condition without referring his cases to a specialist. In addition, there are instructive chapters on stricture, non-gonorrheal urethritis, tumors, urethrorrhea, spermatorrhea, prostatorrhea, impotency, sterility, verumontanitis, and utriculitis.

\section{Coolidge on Nose and Throat}

Manual of Diseases of the Nose and Throat. By Algernon Coolidge, M. D., Professor of Laryngology, Harvard Medical School. Octavo of 360 pages, illustrated. Cloth, \$I.50 net.

\section{READY REFERENCE}

This new book furnishes the student and practitioner a guide and ready reference to the important details of examination, diagnosis, and treatment. Established facts are emphasized and unproved statements avoided. Anatomy and physiology of the different regions are reviewed. 


\section{Braasch's Pyelography}

Pyelography (Pyelo=Ureterography). By William F. BraAsch, M. D., Mayo Clinic, Rochester, Minn. Octavo of 323 pages, with 296 pyelograms. Cloth, $\$ 5.00$ net.

\section{PYELOGRAMS}

This new work is the first comprehensive collection of pyelograms ever issued in book form. The 300 pyelograms included were selected from several thousand made at the Mayo Clinic during the past five years. You get the outlines of normal pelves, those of pathologic conditions, and those of congenitally abnormal pelves. In addition to the pyelograms, you get a descriptive text, intrepreting the outlines, pointing out their great value in diagnosis. You get the history of pyelography and the exact technic-selection of medium to be injected, preparation of solution, method of injection, sources of error, etc. The work is a most complete one, beautifully gotten up, and contains much matter of great diagnostic value.

Published March, 1915

\section{Ogden on the Urine}

Third Edition

Clinical Examination of Urine and Urinary DiagNosis. A Clinical Guide for the Use of Practitioners and Students of Medicine and Surgery. By J. Bergen Ogden, M. D., Medical Chemist to the Metropolitan Life Insurance Company, New York. Octavo, $4 \mathrm{I} 8$ pages, 54 textillustrations, and a number of colored plates. Cloth, $\$ 3.00$ net.

Published October, 1909

"We consider this manual to have been well compiled; and the author's own experience, so clearly stated, renders the volume a useful one both for study and reference."-The Lancet, L.ondon.

\section{Vecki's Sexual Impotence}

Fifth Edition

Sexual Impotence. By Victor G. Vecki, M. D. I 2 mo volume of 400 pages. Cloth, $\$ 2.25$ net.

"A scientific treatise upon an important and much neglected subject. . . The treatment of impotence in general and of sexual neurasthenia is discriminating and judicious." -Johns Hopkins Hospital Bulletin. Published December, 1915 


\section{DeSchweinitz's \\ Diseases of the Eye \\ The New (8th) Edition}

Diseases of the Eye: A HandBook of OpHTHalmic Practice. By G. E. DeSchweinitz, M. D., Professor of Ophthalmology in the University of Pennsylvania, Philadelphia, etc. Handsome octavo of 754 pages, 386 text-illustrations, and 7 chromo-lithographic plates. Cloth, \$6.00 net.

Published June, 1916

\section{WITH 386 TEXT-ILLUSTRATIONS AND 7 COLORED PLATES}

Dr. deSchweinitz's book has long been recognized as a standard authority upon eye diseases, the reputation of its author for accuracy of statement placing it far in the front of works on this subject. For this edition Dr. deSchweinitz has sul,jected his book to a most thorough revision. Many new subjects have been added, a number in the former edition have been rewritten, and throughout the book reference has been made to vaccine and serum therapy, to the relation of tuberculosis to ocular disease, and to the value of tuberculin as a diagnostic and therapeutic agent.

The text is fully illustrateri with black and white cuts and colored plates, and in every way the book maintains its reputation as an authority.

\section{Johns Hopkins Hospital Bulletin}

"No single chapter can be selected as the best. They are all the product of a finished authorship and the work of an exceptional ophthalmologist. The work is certainly one of the best on ophthalmology extant, and probably the best by an American author."

\section{deSchweinitz and Holloway on Pulsating Exophthalmos \\ Published August, 1908}

Pulsating Exopithaluos. An analysis of sixty-nine cases not previously analyzed. By George E. Discineinitz, M. D., and Tiromas B. Holloway, M. D. Octavo of I 25 pages. Cloth, \$2.00 net.

"The book deals very thoroughly with the whole subject, and in it the most com plete account of the disease will be found."-British Medical Journal.

\section{Jackson's Essentials of Eye Fourth Revised Edition}

Essentials of Refraction and of Diseases of the Eie. By Edward Jackson, A. M., M. D., Emeritus Professor of Diseases of the Eye, Philadelphia Polyclinic. Post-octavo of 261 pages, 82 illustrations. Cloth, \$1.25 net. In Saunders' Question-Compend Series.

"The entire ground is covered, and the points that most need careful elucidation are made clear and easy."-Johns Hopkins Hospital Bulletin. Published April, 1906 


\section{GET THE BEST \\ American \\ Illustrated Dictionary}

The New (9th) Edition

The American Illustrated Medical Dictionary. A new and complete dictionary of the terms used in Medicine, Surgery, Dentistry, Pharmacy, Chemistry: Veterinary Science, Nursing, and all kindred branches; with over roo new and elaborate tables and many handsome illustrations. By W. A. Newman DoRLAND, M.D. Large octavo of I 79 pages. Flexible leather, $\$ 5.00$ net; with thumb index, $\$ 5.50$ net. Published September, 1917

\section{OVER 2000 NEW WORDS IN THIS EDITION}

For this edition the book has been subjected to a thorough revision and entirely reset, adding thousands of important new terms. This work is more than a medical dictionary-it is a Medical Encyclopedia.

\section{Howard A. Kelly, M.D.,}

Professor of Gynecologic Surgery, Johns Hopkins University, Baltimore

"Dr. Dorland's Dictionary is admirable. It is so well gotten up and of such convenient size. No errors have been found in my use of it."

\section{Pilcher's Practical Cystoscopy}

Practical Cystoscopy. By Paul M. Pilcher, M. D., formerly of the Eastern Long Island Hospital. Octavo of 504 pages, with 299 illustrations, 29 in colors. Cloth, \$6.00 net.

\section{SECOND EDITION-published November, 1915}

To be properly equipped, you must have at your instant command the information this book gives you. It explains away all difficulty, telling you why you do not see something when something is there to see, and telling you how to see it. All theory has been uncompromisingly eliminated, devoting every line to practical, needed every-day facts, telling you how and when to use the cystoscope and catheter-telling you in a way to make you know.

Bransford Lewis, M. D., St. Louis University best in the English language now." 


\section{Haab and DeSchweinitz's} External Diseases of the Eye

(Published February, 1909)

Atlas and Epitome of External Diseases of the Eye. By Dr O. HAAB, of Zïrich. Edited, with additions, by G. E. DESchweinitz, M. D., Professor of Ophthalmology, University of Pennsylvania. Ior colored illustrations and 244 pages of text. Cloth, $\$ 3.00$ net.

Third Edition.

Saunders' Atlases.

\section{Stokes' The Third Great Plague}

The Third Great Plague: A Discussion of Syphilis for Every=day People. By Joun H. Stokes, A. B., M. D., Head of Section on Dermatology and Syphilology, Division of Medicine, The Mayo Clinic. I 2 mo of 204 pages. Cloth, \$r.50 net.

Published October, 1917

\section{American Pocket Dictionary New (10th) Edition}

Tue American Pocket Medical Dictionary. Edited by W. A. Newman Dorland, M. D. Containing the definition of the principal words used in medicine and kindred sciences. 707 pages. Flexible leather, with gold edges, \$I.25 net; with thumb index, \$1.50 net.

"I am struck at once with admiration at the compact size and attractive exterior. I can recommend it to our sturlents without reserve."-JAMES W. Holland, M. D., Emeritus Professor of Medical Chemistry and Toxicology at the Jefferson Medical College, $\begin{array}{ll}\text { Philadelphia. } & \text { Published September, } 1917\end{array}$

\section{Jackson on the Eye Preparing-New (3d) Edition}

A Mandal of the Diagnosis and Treatment of Diseases of the. Eye. By Enward Jackson, A. M., M. D., Professor of Ophthalmology, University of Colorado. I 2 mo of 6 I 5 pages, with I 84 illustrations. 


\section{Barnhill and Wales' Modern Otology}

A Text-Book of Modern Otology. By John F. BarnHiLl, M. D., Professor of Otology, Laryngology, and Rhinology, and Earnest DE W. Wales, M. D., Clinical Professor of Otology, Laryngology, and Rhinology, Indiana University School of Medicine, Indianapolis. Octavo of 598 pages, with 314 original illustrations. Cloth, $\$ 5.50$ net.

\section{SECOND EDITION}

This work represents the results of personal experience as practitioners and teachers, influenced by the instruction given by such authorities as Sheppard, Dundas Grant, Percy Jakins, Jansen, and Alt. Much space is devoted to prophylaxis, diagnosis, and treatment, both medical and surgical. There is a special chapter on the bacteriology of ear affections-a feature not to be found in any other work on otology. Great pains have been taken with the illustrations. A large number represent the best work of Mr. H. F. Aitken.

Frank Allport, M. D.,

Professor of Otology, Northwestern University, Chicago.

"I regard it as one of the best books in the English language on this subject. The pictures are especially good, particularly as they are practically all original and not the old reproduced pictures so frequently seen."

Published January, 1911

\section{Davis' Accessory Sinuses}

\section{Development and Anatomy of the Nasal Accessory} Sinuses in Man. By Warren B. Davis, M. D., Corinna Borden Keen Research Fellow of the Jefferson Medical College, Philadelphia. Octavo of 172 pages, with 57 original illustrations. Published March, 1914

\section{ORIGINAL DISSECTIONS}

This book is based on the study of two hundred and ninety lateral nasal walls, presenting the anatomy and physiology of the nasal accessory sinuses from the sixtieth day of fetal life to advanced maturity. It was necessary for Dr. Davis to develop a new technic by which the accessory sinus areas could be removed en masse at the time of postmortem examinations, and still permit of reconstruction of the face without marked disfigurement. 


\section{Greene and Brooks' Genito-Urinary Diseases}

A 'Text-Book of Genito-Urinary Diseases. By RoBert H. GreEne, M.D., Professor of Genito-Urinary Surgery at Fordham University; and Harlow Brooks, M. D., Assistant I'rofessor of Clinical Medicine, University and Bellevue Hospital Medical School. Octavo of 666 pages, illustrated. Cloth, $\$ 5.50$ net.

\section{FOURTH EDITION-published May, 1917}

This new work covers completely the subject of genito-urinary diseases, presenting both the medical and surgical sides. Kidney diseases are very elaborately detailed.

\section{New York Medical Journal}

"As a whole the book is one of the most satisfactory and useful works on genitourinary diseases now extant, and will undoubtedly be popular among practitioners and students."

\section{Gleason on Nose, Throat, and Ear}

A Manual of Diseases of the Nose, Throat, and Ear. By E. Baldwin Glzason, M. D., LL. D., Professor of Otology, Medico-Chirurgical College, Graduate School of Medicine, University of Pennsylvania, Philadelphia. I $2 \mathrm{mo}$ of 590 pages, profusely illustrated. Cloth, $\$ 2.75$ net.

Published October, 1914

\section{THIRD EDITION}

Methods of treatment have been simplified as much as possible, so that in most instances only those methods, drugs, and operations have been advised which have proved essential. A feature consists of the collection of formulas.

\section{American Journal of the Medical Sciences}

"For the practitioner who wishes a reliable guide in laryngology and ot ology there ar few books which can be more heartily commended."

\section{Wilcox on Genito-Urinary and Venereal Dis-}

eases Second Edition, published January. 1909

Essentiats of Genito-Urinary and Venereal Diseases. By Starling S. Wilcox, M. D., Lecturer on Genito-Urinary Diseases and Syphilology, Starling-Ohio Medical College, Columbus, Ohio. I $2 \mathrm{mo}$ of 321 pages, illustrated. Cloth, \$1.25 net. In Saunders' Question-Compends. 


\section{Head's Modern Dentistry}

Modern Dentistry. By Joseph Head, M. D., D.D.S., Dentist to the Jefferson Hospital, Philadelphia. Octavo of 374 pages, with 309 illustrations. Cloth, $\$ 5.00$ net.

Published December, 1917

Dr. Head's book is a complete and up-to-date text-book on dentistry. It gives you the principles upon which successful work must be based-. the technic in full, and the results of original experiments, with formulæ, instruments, and methods. It brings out clearly the various factors which influence the diagnosis, and carefully details the methods of treatment. The subject of vaccines is gone into very fully, giving you preparation and use of autogenous, stock, and special vaccines. Particularly useful are the sections on mouth hygiene, local anesthesia by novocain, electrolysis, tooth discoloration, care of children's teeth and gums, orthodontia for the general practitioner of dentistry, cement, $x$-ray study, and the use of emetin. Over three hundred original illustrations show the student just how the procedures are to be carried out.

\section{Kyle's Nose and Throat}

Diseases of the Nose and Throat. By D. Braden Kyle, M.D., formerly Professor of Laryngology in the Jefferson Medical College, Philadelphia; Consulting Laryngologist, Rhinologist, and Otologist, St. Agnes' Hospital. Octavo, $85^{6}$ pages; with 272 illustrations and 27 lithographic plates in colors. Cloth, $\$ 4.50$ net.

Published November, 1914

\section{FIFTH EDITION}

This work has now reached its fifth edition. With the practical purpose of the book in mind, extended consideration has been given to treatment, each disease being considered in full, and definite courses being laid down to meet special conditions and symptoms.

\section{Pennsylvania Medical Journal}

“ Dr. Kyle's crisp, terse diction has enabled the inclusion of all needful nose and throat knowledge in this book. 'The practical man, be he special or general, will not search iti vain for anything he needs." 


\section{Holland's}

\section{Chemistry and Toxicology}

A Text-Book of Medical Chemistry and Toxicology. By James W. Holland, M. D., Emeritus Professor of Medical Chemistry and Toxicology, Jefferson Medical College, Philadelphia. Octavo, 678 pages, illustrated. Cloth, $\$ 3.00$ net.

\section{FOURTH EDITION-published April, 1915}

Dr. Holland's work is an entirely new one, and is based on his thir:y-five years' practical experience in teaching chemistry and medicine. Recognizing that to understand physiologic ehemistry students must first be informed upon points not referred to in most medical text-books, the at thor has included in his work the latest views of equilibrium of equations, mass-action, cryoscopy, osmotic pressure, etc. Much space is given to toxicology.

\section{American Medicine}

"Its statements are clear and terse; its illustrations well chosen; its development logical, systematic, and comparatively easy to follow. . . . We heartily commend the work."

\section{Ivy's Applied Anatomy and Oral Surgery}

Applied Anatomy and Oral Surgery for Dental Students. By Robert H. Ivy, M. D., 1). D. S., Assistant Oral Surgeon to the Philadelphia General Hospital. I 2 mo of 290 pages, illustrated. Cloth, , \$1.75 net.

Second Edition published July, 1917

This work is just what dental students have long wanted-a concise, practical work on applied anatomy and oral surgery, written with their needs solely in mind. No one could be better fitted for this task than Dr. Ivy, who is a graduate in both dentistry and medicine. The text is well illustrated with pictures that you will find extremely helpful.

"I am delighted with this compact little treatise. It seems to me just to fill the bill."-H. P. KunN, M. D., Western Dental College, Kansas City.

\section{Oertel on Bright's Disease}

The Anatomic Histological Processfs of Brigitt's Disease. By Horst Oertel, M. D., Director of the Russell Sage Institute of Pathology, New York. Octavo of 227 pages, with 44 illustrations and 6 colored plates. Cloth, $\$ 5.00$ net.

These lectures deal with the anatomic histological processes of Bright's disease, and In a somewhat different way from the usual manner. Everywhere relations are emphasized and an endeavor made to reconstruct the whole as a unit of interwoven processes.

Published December, 1910

“Dr. Oertel gives a clear and intelligent idea of nephritis as a continuous process. "We can strongly recommend this book as thoughtful, scientific, and suggestive."The Lancet, London. 


\section{Goepp's Dental State Boards}

Dental State Board Questions and Answers. By $R$. Max Goepp, M. D., Professor of Clinical Medicine at the Philadelphia Polyclinic. Octavo of 428 pages. Cloth, $\$ 3.00$ net.

\section{SECOND EDITION}

This new work is along the same practical lines as Dr. Goepp's successful work on Medical State Boards. The questions included have been gathered from reliable sources, and embrace all those likely to be asked in any State Board examination in any State. They have been arranged and classified in a way that makes for a rapid résumé of every branch of dental practice, and the answers are couched in language unusually explicit-concise, definite, accurate.

Published February, 1916

\section{McConnell's Pathology and Bacteriology}

Dental

General Pathology and Bacteriology for Dental Students. By Guthrie McConnell, M. D., Assistant Surgeon, Medical Reserve Corps, U. S. N. I 2 mo of 314 pages, illustrated.

Second Edition published January, 1918

This work was written expressly for dentists and dental students, emphasizing throughout the application of pathology and bacteriology in dental study and practice. There are chapters on disorders of metabolism and circulation; retrogressive processes, cell division, inflammation and regeneration, granulomas, progressive processes, tumors, special mouth pathology, sterilization and disinfection, bacteriologic methods, specific micro-organisms, infection and immunity, and laboratory technic.

\section{Bliss' Qualitative Chemical Analysis}

Qualitative Chemical Analysis. By A. R. Bliss, Jr., M.D., PH.G., Professor of Pharmacology, Emory University, Atlanta, Georgia. I 12 mo of 194 pages. Cloth, \$2.25 net.

Second Edition published May, 1918

This work was prepared especially for medical, dental and pharmacy students, and laboratory workers in these fields. It gives you systematic procedures for the detection and separation of the most common bases and acids, and in such a manner that, in a short time, the student will be enabled to gain a good practical knowledge of the theory and methods of analysis. 


\section{Bass and Johns' Alveolodental Pyorrhea}

Alveolodental Pyorrhea. By Ciharles C. Bass, M.D., Professor of Experimental Medicine, and Foster M. Johss, M. D., Insteuctor in the Laboratories of Clinical Medicine, Tulane Medical College. Octavo of 168 pages, illustrated. Cloth, \$2.50 net. Published June, 1915

This work discusses alveolodental pyorrhea from the viewpoint of infection by the Endamœba buccalis in a simple, concise way, in the light of recent information.

\section{Gleason's Nose and Throat Fourth Edition, Revised}

Essentials of Diseases of the Nose and Tiroat. By E. B. GLEason, S.B., M.D., Clinical Prof essor of Otology, Medico-Chirurgical College, Graduate School of Medicine, University of Pennsylvania, Post-octavo, 24I pages, II 2 illustrations. Cloth, \$I.25 net. In Saunders' Question-Compend Series.

Published October, 1914

"The careful description which is given of the various procedures would be sufficient to enable most people of average intelligence and of slight anatomical knowledge to make a very good attempt at laryngoscopy."-The Lancet, London.

\section{Norris' Gonorrhea in Women}

Gonorrhea in Women. By Charles C. Norris, M. D., Instructor in Gynecology, University of Pennsylvania. Introduction by JoHN G. Clark, MI. D., University of Pennsylvania. Octavo of $52 \mathrm{I}$ pages, illustrated. Cloth, \$6.50 net.

Published May, 1913

\section{Preiswerk and Warren's Dentistry}

Atlas and Epitome of Dentistry. By Prof. G. Preiswerk, of Basil. Edited, with additions, by GEorge W. WARREN, D. D. S., Professor of Operative Dentistry, Pennsylvania College of Dental Surgery, Philadelphia. With 44 lithographic plates, 152 text-cuts, and 343 pages of text. Cloth, \$3.50 net. Saunders' Hand-Atlases. August, 1906

\section{Grünwald and Grayson on the Larynx}

Atlas and Epitome of Diseases of the I,arynx. By Dr. I. GrüNWALD, of Munich. Edited, with additions, by CHARLes P. Grayson, M. D., University of Pennsylvania. With IO7 colored figures on 44 plates, 25 text-cuts, and 103 pages of text. Cloth, $\$ 2.50$ net. In Saunders' Itand-Atlas Series.

Published 1898

\section{Mracek and Stelwagon's Atlas of Skin Second}

Atlas and Epitome of Diseasfs of the Skin. By Prof. Dr. Franz Mracek, of Vienna. Edited, with additions, by HeNry W. Stelwagon, M. D., Jefferson Medical College. With. 77 colored plates, 50 half-tone illustrations, and 280 pages of text. Cloth, $\$ 4.00$ net. In Saunders' Hand-Atlas Series. 


\section{Theobald's \\ Prevalent Diseases of the Eye}

Prevalent Diseases of the Eye. By Samuel Theobald, M. D., Clinical Professor of Ophthalmology and Otology, Johns Hopkins University. Octavo of $55^{\circ}$ pages, with 2 I 9 text-illustrations and ro plates. Cloth, $\$ 4.50$ net.

Published July, 1906

Chas. A. Oliver, M. D.,

Clinical Professor of Ophthalmology, Woman's Medical College, Phila.

"I feel I can conscientiously recommend it, not only to the general physician and medical student, but also to the experienced ophthalmologist."

\section{Wells' Chemical Pathology}

Chemical Pathology. By H. Gideon Wells, Ph.D., M. D., Professor of Pathology in the University of Chicago. Octavo of 707 pages. Cloth, $\$ 4.25$ net.

Wm. H. Welch, M.D., Johns Hopkins University.

Third Edition

Published February, 1918

"The work fills a real need in the English literature of a very important subject, and I shall be glad to recommend it to my students."

\section{Stelwagon's Essentials of Skin}

Seventh Edition

Essentials of Diseases of the Skin. By Henry W. Stelwagon, M. D., PH. D., Professor of Dermatology in the Jefferson Medical College, Philadelphia. Post-octavo of $29^{2}$ pages, with 72 text-illustrations and 8 plates. Cloth, \$r.25 net. In Saunders' Question-Compend Series.

Published August, 1909

"In line with our present knowledge of diseases of the skin. . . . Continues to maintain the high standard of excellence for which these question compends have been noted."-The Medical News. 



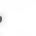

$-$ 




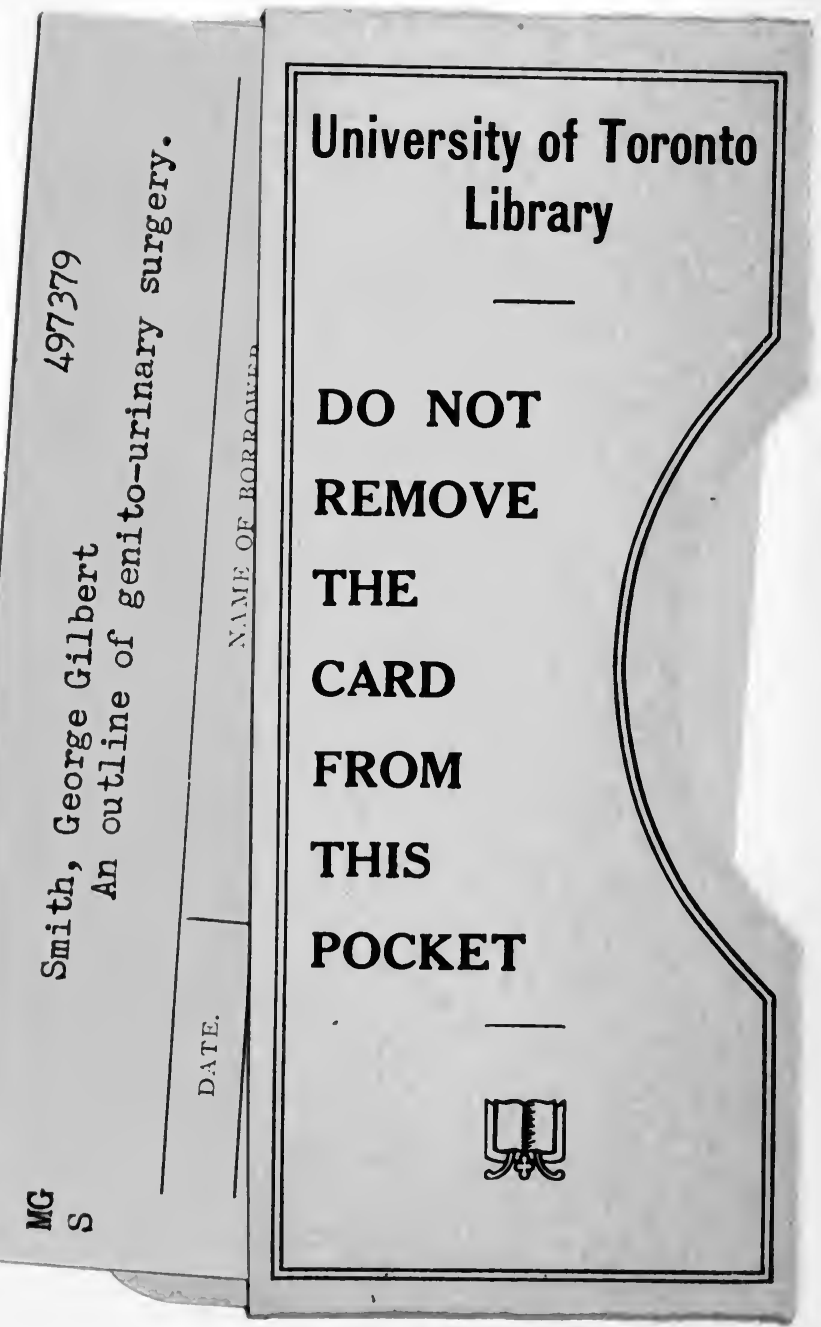


